\title{
AMPLIFIED PHOTOCHEMISTRY WITH SLOW PHOTONS
}

\author{
by
}

Jennifer I-Ling Chen

A thesis submitted in conformity with the requirements

for the degree of Doctor of Philosophy

Department of Chemistry

University of Toronto

(C) Copyright by Jennifer I-Ling Chen, 2009 


\title{
Amplified Photochemistry with Slow Photons
}

\author{
Jennifer I-Ling Chen \\ Ph. D., Department of Chemistry, University of Toronto, 2009
}

\begin{abstract}
Slow photon, or light with reduced group velocity, is a unique phenomenon found in photonic crystals that theoreticians have long suggested to be invaluable for increasing the efficiency of light-driven processes. This thesis demonstrates experimentally the feasibility of using slow photons to optically amplify photochemistry of both organic and inorganic systems. The effect of photonic properties on organic photochemistry was investigated by tracing out the wavelength-dependent rate of photoisomerization of azobenzene anchored on silica opals. The application of slow photons to inorganic photochemical processes was realized by molding nanocrystalline titania into an inverse opal structure and investigating its photodegradation efficiency in relation to the photonic properties. Changes in the photodegradation efficiency were directly linked to modifications of the electronic band gap absorption as a result of the photonic properties. The highest enhancement of twofold was achieved when the energy of the slow photons overlaps with the electronic band gap absorption, such that the loss of light due to photonic stop-band reflection was significantly reduced. In addition, the strength of slowphoton amplification with respect to the macroscopic structural order was studied by
\end{abstract}


introducing controlled disorder via the incorporation of guest spheres into the opal templates. For the first time, a correlation between structural order, photonic properties and a photochemical process was established. The ability to combine slow-photon optical amplification with chemical enhancement was further achieved by incorporating platinum nanoparticles in inverse titania opals where the platinum nanoparticles increased the lifetimes of the higher population of electron-hole pairs arising from slow photon. Overall, various important factors governing the slow photon enhancement were investigated in detail, including the energy of the photonic stop band, angle dependence, thickness of the film, degree of structural order, filling fraction of the dielectric material and diffusion of a second medium if present. Theoretical calculations based on scalarwave approximation in support of the experimental findings were provided wherever possible. The findings provide a blueprint for achieving optical amplification using slow photons in the broad range of photochemical or photophysical processes. 


\section{Acknowledgements}

I thank Professor Geoffrey Ozin for the inspiration, freedom, support and guidance to my doctoral research. More importantly, I thank him for the unrelenting encouragements, on both scientific and personal levels, that he has provided throughout the past four years. I am further indebted to him for the great opportunity to work in such a diverse interdisciplinary research group that has widened my knowledge and perspective on materials chemistry tremendously. Above all, I have learned much from his creative thinking and approach to research, which I hope to emulate in my future endeavors.

Several co-workers were indispensable to the completion of my doctoral work. I am deeply indebted to Professor Vladimir Kitaev for his innumerable vital suggestions that have lead to the success of my projects. I also thank him for being a great friend who continuously encouraged me, in addition to being a selfless colleague who put my interests before his own on many occasions. I thank Sue Mamiche for all her assistance to Geff in managing group businesses and the help in some engineering aspects of my projects. Furthermore I appreciate her friendship, company, and womanly advice that are sometimes hard to find in a group that is male-dominated.

Of the other collaborators, I thank Sung Yeun Choi for her introduction to titania chemistry and photocatalysis experiments; Dr. Georg von Freymann for the introduction to the theory of slow photons and help on theoretical calculations; three undergraduate students, Zhuo Li, Edward Loso and Naazia Ebrahim, for their work on doped and metal- 
loaded titania inverse opals. I also thank Dave Rider and Mark Wheeler for the opportunity to collaborate on polymer and metamaterial projects, respectively, which has been a great learning experience for me.

During the course of my research, I received a great amount of assistance from many technical staff: Ilya Gourevich, Neil Coombs and Sal Boccia for SEM and STEM; Srebri Petrov for XRD; Rana Sodhi for XPS; Milos Kucera for Raman and Profilometry; and Dan Mathers for GC and ICP-AES. I also thank Alexei Esmanski and Takashi Suezaki for their assistance with RIE.

Past and present Ozin group members have made the last four years enjoyable and scientifically rewarding. I thank all of the support, friendship and understanding that they have provided. As for certain I would miss out some names of the 30+ people whom I have come to know, I shall not try to list them all. However I will mention a few who have made my first experience of living-away-from-home particularly interesting and joyful. I thank Freddy for her super energetic attitude in research and life that has always cheered me up and often brought me laughing to tears. I treasure the times we spent in the lab working like crazy in the first two years of my $\mathrm{Ph}$. D., the many interesting experiences we had embarked on in and around Toronto, some (attempts of) cultural exchange in terms of language and cooking, and till this day the almost weekly two-hour conversations over the internet that continue to provide me laughter, awe, advice and scientific insights. In the latter course of my Ph. D., I am fortunate to have Taka as a great colleague and friend, who has taught me a great deal about solar cells in addition to being enthusiastic of the various sports activities and weekly dine-outs. I also appreciate Wes' help with crosswords during lunch time, John's shared love of classical music, Kun's 
deep, yet often animated, philosophical discussions of Chinese culture and the participation of many people in badminton that often provided relief to my stress and frustrations related to research. Outside the lab, I give my highest gratitude to the Wilhelm family who provided me not only shelter but also love and care since my first arrival in Toronto. I had never expected such a heartwarming experience away from home.

Lastly I thank the people who are the most important to me - my family and my fiancé. I thank my parents for the years of care and sacrifice they have made to provide the education and life I am privileged to have. I also thank their open-mindedness to allow me to discover my own path and their continuous belief in my ability. I thank my fiancé Aaron for his patience, understanding and support of my academic pursuit thousands of kilometers away from him. He has given me a peace of mind in pursuing my dream as I know he is always there for me. I am indebted to his hard work for achieving security for our future - a new chapter in life that I am most eager to begin. 


\section{Table of Contents}

Table of Contents vii

List of Figures $\quad$ xiii

List of Schemes and Tables $\quad$ xxii

CHAPTER 1 - Introduction 1

1.1 Photonic Crystal 1

1.1.1. Three-dimensional Colloidal Photonic Crystal 4

1.2 Theory of Photonic Band Diagram 7

1.2.1 Group Velocity and Density of States 9

1.2.2 Absorption and Non-linear Optical Properties in Photonic Crystal 11

1.3 Scalar-wave Approximation for Modeling Photonic Properties 13

1.4 Slow Photons in Opals and Inverse Opals 16

1.5 Examples of Slow Photon and Photonic Band-edge Enhancement 17

1.6 Recent Progress on Incorporating Photonic Structures in Photovoltaic Cell 18

$\begin{array}{lll}1.7 & \text { References } & 19\end{array}$

CHAPTER 2 - Tracing the Effect of Slow Photon in Photoisomerization of

$\begin{array}{ll}\text { Azobenzene } & 24\end{array}$

$\begin{array}{lll}2.1 & \text { Introduction } & 25\end{array}$

$\begin{array}{lll}2.2 & \text { Experimental Methods } & 28\end{array}$ 
2.2.1 Fabrication of Azobenzene-functionalized $\mathrm{SiO}_{2}$ Opal 28

2.2.2 Photoisomerization Experiment 29

2.3 Photoisomerization of Azobenzene Anchored on $\mathrm{SiO}_{2}$ Opal 30

2.3.1 Effect of Slow Photons and Photonic Stop Band 35

2.3.2 Effect of Thickness of Opal 36

2.3.3 Effect of Azobenzene Packing Density 40

$\begin{array}{lll}2.4 & \text { Conclusions } & 42\end{array}$

$\begin{array}{lll}2.5 & \text { References } & 42\end{array}$

CHAPTER 3 - Slow Photon Enhanced Photodegradation Efficiency of

$\begin{array}{ll}\text { Titania } & 45\end{array}$

$\begin{array}{lll}3.1 & \text { Introduction } & 46\end{array}$

3.1.1 Optical and Electronic Properties of Anatase $\mathrm{TiO}_{2}$

3.1.2 Photocatalysis with Anatase $\mathrm{TiO}_{2} \quad 49$

3.1.3 Surface Chemistry of $\mathrm{TiO}_{2} \quad 50$

3.1.4 Enhancing the Absorption of Anatase $\mathrm{TiO}_{2}$

3.2 Experimental Methods $\quad 52$

3.2.1 Fabrication of Inverse $\mathrm{TiO}_{2}$ Opals $\quad 52$

3.2.2 Fabrication of Reference Nanocrystalline $\mathrm{TiO}_{2}$

3.2.3. Materials Characterization 53

3.2.3.1 Scanning (Transmission) Electron Microscopy 53

3.2.3.2 X-ray Diffraction and Raman Spectroscopy 54

3.2.3.3 UV-Vis Spectroscopy $\quad 54$ 
3.2.4 Dye Adsorption Study $\quad 54$

3.2.5 Photodegradation Experiment 55

3.3 Characterization of Inverse $\mathrm{TiO}_{2}$ Opals 55

3.3.1 Optical Properties $\quad 55$

$\begin{array}{lll}\text { 3.3.2 Structural Morphology } & 60\end{array}$

$\begin{array}{lll}\text { 3.3.3 Crystal Phase } & 62\end{array}$

3.4 Solid-state Photodegradation Efficiency 63

3.4.1 Adsorption of Methylene Blue 64

3.4.2 Monochromatic Irradiation 65

3.4.3 Effect of Incidence Irradiation Angle $\quad 68$

3.4.4 White Light Irradiation $\quad 69$

$\begin{array}{lll}3.5 & \text { Conclusions } & 72\end{array}$

$\begin{array}{lll}3.6 & \text { References } & 73\end{array}$

CHAPTER 4 - Effect of Disorder on the Photodegradation Efficiency of $\begin{array}{ll}\text { Inverse Titania Opals } & 76\end{array}$

$\begin{array}{lll}4.1 & \text { Introduction } & 77\end{array}$

$\begin{array}{llr}4.2 & \text { Experimental Methods } & 80\end{array}$

4.3 Characterization of Binary Polystyrene Opals and Inverse $\mathrm{TiO}_{2}$ Opals 81

4.3.1 Optical Properties of Binary Polystyrene Opal Templates 82

4.3.2 Domain Size Analysis on (1-x)150-x180 Opal Templates 83

4.3.3 Optical Properties of Binary Inverse $\mathrm{TiO}_{2}$ Opals 86

4.3.4 Structure of Binary Inverse $\mathrm{TiO}_{2}$ Opals 89 
4.4 Photodegradation by Disordered Inverse $\mathrm{TiO}_{2}$ Opals 92

4.4.1 Binary Systems of $(1-x) 150-x 180$ and $(1-x) 150-x 210$

4.4.2 Effect of Light Scattering 96

$\begin{array}{lll}4.5 & \text { Conclusions } & 98\end{array}$

$\begin{array}{llr}4.6 & \text { References } & 99\end{array}$

CHAPTER 5 - Heterogeneous Photocatalysis with Inverse Titania Opals 101

$\begin{array}{llr}5.1 & \text { Introduction } & 102\end{array}$

$\begin{array}{lll}5.2 & \text { Experimental Methods } & 103\end{array}$

5.2.1 Increasing the Filling Fraction of $\mathrm{TiO}_{2} \quad 103$

5.2.2 Determination of the Mass of Films 103

5.2.3 Specific Surface Area from Dye Adsorption-Desorption 103

$\begin{array}{lll}\text { 5.2.4 Solution Photocatalysis } & 104\end{array}$

5.3 Effect of Filling Fraction on the Group Velocity 104

5.4 Characterization of Inverse $\mathrm{TiO}_{2}$ Opals with Higher Filling Fraction 105

$\begin{array}{lll}\text { 5.5 Photocatalysis in Solution Phase } & 108\end{array}$

5.5.1 Effects of Surface Area and Diffusion 108

5.5.2 Rate of Degradation by As-Prepared Inverse $\mathrm{TiO}_{2}$ Opals 110

5.5.3 Rate of Degradation by Inverse $\mathrm{TiO}_{2}$ Opals with Higher Filling $\begin{array}{ll}\text { Fraction } & 111\end{array}$

$\begin{array}{lll}5.6 & \text { Conclusions } & 113\end{array}$

$\begin{array}{lll}5.7 & \text { References } & 113\end{array}$ 
CHAPTER 6 - Synergy of Slow Photon and Chemically Amplified

Photochemistry in Platinum-loaded Inverse Titania Opals

$\begin{array}{lll}6.1 & \text { Introduction } & 116\end{array}$

$\begin{array}{lll}6.2 & \text { Experimental Methods } & 118\end{array}$

6.2.1 Fabrication of Pt-loaded Inverse $\mathrm{TiO}_{2}$ Opals 118

6.2.2 X-ray Photoelectron Spectroscopy 118

6.2.3 Dye Adsorption and Photodegradation 119

6.3 Photodeposition and Characterization of Pt-loaded Inverse $\mathrm{TiO}_{2}$ Opals 119

6.3.1 Morphological Study of Pt Clusters by STEM 120

$\begin{array}{lll}\text { 6.3.2 Chemical Analysis by XPS } & 121\end{array}$

$\begin{array}{lll}\text { 6.3.3 Optical Properties } & 124\end{array}$

6.4 Adsorption of Acid Orange on Pt-loaded Inverse $\mathrm{TiO}_{2}$ Opals 125

6.5 Photodegradation Efficiency of Pt-loaded Inverse $\mathrm{TiO}_{2}$ Opals 126

$\begin{array}{lll}6.6 & \text { Conclusions } & 130\end{array}$

$\begin{array}{lll}6.7 & \text { References } & 130\end{array}$

CHAPTER 7 - Outlook and Future Directions 132

7.1 Application of Inverse $\mathrm{TiO}_{2}$ Opals in Sterilizing Light Bulbs 132

7.1.1 Gas-phase Photocatalysis Using Inverse $\mathrm{TiO}_{2}$ Opals and Incandescent Light Bulb

7.1.2 Prototype Light Bulb Coated with Inverse $\mathrm{TiO}_{2}$ Opal 134

7.2 Other Photonic Structures for Optical Enhancement 137

7.2.1 Tandem Structure of Multiple Inverse $\mathrm{TiO}_{2}$ Opals 
7.2.2 Bi-layer Structure Comprising a Non-absorbing Photonic Crystal

7.3 References 


\section{LIST OF FIGURES}

\section{CHAPTER 1}

Figure 1.1. Photographs and scanning electron images showing the submicron periodic structure of natural photonic crystals: opal gem stones (a), butterfly wings (b) and beetle scales (c).

Figure 1.2. Examples of artificial one-, two- and three-dimensional photonic crystals comprised of: alternating layers of porous $\mathrm{Si}(\mathrm{a})$; cylindrical holes in $\mathrm{Si}$ (b) and woodpile structure of chalcogenide sulfide (c).

Figure 1.3. Powders of inverse colloidal crystals of various periodicities obtained from sedimentation (a) and single-crystal opal films obtained from evaporation-induced self-assembly (b).

Figure 1.4. Photonic band diagram of an inverse Si opal showing the dispersion relation between energy of light and the wave vector.

Figure 1.5. A comparison of total DOS (a) and local DOS (b and c) for a fcc structure of $\mathrm{TiO}_{2}$ spheres in water. The local DOS calculated at midway between two adjacent spheres (b) and at the center of sphere (c).

Figure 1.6. Extinction spectra of a 27-layer opal of $205 \mathrm{~nm}$ spheres measured experimentally (solid black line) vs calculated by SWA (grey dashed-line) assuming sphere size of $205 \mathrm{~nm}$, and $15 \%$ of hydration layer in the void space.

Figure 1.7. Simplified photonic band diagram of a photonic crystal (a). The position of the slow photons, indicated by the circles, with respect to the stop band reflection is shown in (b). 
Figure 1.8. Different photonic structures for increasing the path length of light: (a) an inverse $\mathrm{TiO}_{2}$ opal on the back of a thick $\mathrm{TiO}_{2}$ absorbing layer as a wavelengthselective back reflector (an increase in the photocurrent seen on the right); and (b) a designed 2D grating with distributed Bragg reflector at the back of a silicon film.

\section{CHAPTER 2}

Figure 2.1. Examples of applications of azobenzene: (a) photoinduced microemulsion of heptane and water using azobenzene derived surfactant; (b) direct bending of a liquid-crystal polymer film containing azobenzene moiety under uni-directional irradiation; (c) photoisomerization of azobenzeneincorporated DNA for controlled duplex formation and (d) photoresponsive iontransport membrane.

Figure 2.2. Absorption spectra of trans- (E) and cis- $(Z)$ azobenzene in ethanol.

Figure 2.3. Extinction spectra of reference $\mathrm{SiO}_{2}-\mathrm{O}$ of $85 \mathrm{~nm}$ sphere size (a), 318$\mathrm{SiO}_{2}-\mathrm{O}$ (b) and 380-SiO $2-\mathrm{O}$ (c) prior to functionalization (black), after reacting with MPTES (red) and with 4-phenylazomaleinanil (blue).

Figure 2.4. Absorbance of 380-azo- $\mathrm{SiO}_{2}-\mathrm{O}$ (open) and reference azo- $\mathrm{SiO}_{2}-\mathrm{o}$ (filled) films after background subtraction with respect to the concentration of (3mercaptopropyl)-trimethoxysilane.

Figure 2.5. Evolution of the absorbance spectra of azo-SiO azo-SiO $2-\mathrm{o}$ (b) and 380-azo-SiO$-\mathrm{SiO}_{2}$ (c) under UV-irradiation after background subtraction (inset shows the extinction of the films).

Figure 2.6. Absorbance of azo- $\mathrm{SiO}_{2}-\mathrm{O}$ at $330 \mathrm{~nm}$ as a function of $\int \frac{1-10^{-\mathrm{A}^{\lambda}(\mathrm{t})}}{\mathrm{A}^{\lambda}(\mathrm{t})} \mathrm{dt}$ 
Figure 2.7. Wavelength-dependent enhancement factor for the trans-cis photoisomerization of 318-azo- $\mathrm{SiO}_{2}-\mathrm{o}$ and $380-\mathrm{azo}_{-} \mathrm{SiO}_{2}-\mathrm{o}$ (open and solid circles respectively).

Figure 2.8. Group delay for opals with 7 (a), 13 (b) and 19 (c) layers.

Figure 2.9. Enhancement factor for $380-\mathrm{azo}_{-} \mathrm{SiO}_{2}-\mathrm{o}$ of various thicknesses under $355 \mathrm{~nm}$ irradiation in comparison to a reference azo- $\mathrm{SiO}_{2}-\mathrm{o}$.

Figure 2.10. A plot of the difference in the absorbance at PSS and initial state as a function of the number of layers. The linear fit is $y=0.0093+0.0026 x$.

Figure 2.11. Photoswitchable gold substrate can be obtained from a mixedmonolayer containing azobenzene alkanethiol and spacer molecule (a). An optimized photoswitchable gold nanoparticle system using unsymmetrical azobenzene disulfides (b).

Figure 2.12. Absorbance of the films and reversibility of azobenzene on $\mathrm{SiO}_{2}$ opal of $85 \mathrm{~nm}$ spheres after dilution with the spacer.

\section{CHAPTER 3}

Figure 3.1. Crystal structures of the polymorphs of $\mathrm{TiO}_{2}$.

Figure 3.2. Graphical representation of the enthalpy of nanocrystalline $\mathrm{TiO}_{2}$ with respect to bulk rutile as a function of surface area.

Figure 3.3. Energy level band diagram of anatase $\mathrm{TiO}_{2}$ showing the electronic transitions between the valence and conduction band.

Figure 3.4. Illustration of the proposed mechanism of chemisorption of $\mathrm{H}_{2} \mathrm{O}$ on the surface of $\mathrm{TiO}_{2}$ to yield charge-active hydroxyl groups. 
Figure 3.5. Different approaches for increasing the path length of light through $\mathrm{TiO}_{2}$ via random light scattering in photonic sponge structure (a) and incorporation of spherical voids (b).

Figure 3.6. Extinction spectra of opal templates made from different polystyrene spheres (size indicated in the legend).

Figure 3.7. Extinction spectra of bare, infiltrated and inverse opal made from $210 \mathrm{~nm}$ spheres.

Figure 3.8. Reflectance spectra of $\mathrm{i}-\mathrm{nc}-\mathrm{TiO}_{2}-\mathrm{o}$ in air (a) and water (b).

Figure 3.9. Linear relationship between stop band position and template sphere size.

Figure 3.10. Absorbance of $\mathrm{i}-\mathrm{nc}-\mathrm{TiO}_{2}-\mathrm{o}$ measured using an integrating sphere (a). Changes in absorbance of $\mathrm{i}-\mathrm{nc}-\mathrm{TiO}_{2}-\mathrm{O}$ with photonic effects in the UV range with respect to $280-\mathrm{i}-\mathrm{nc}-\mathrm{TiO}_{2}-\mathrm{o}(\mathrm{b})$.

Figure 3.11. SEM images of: $345-\mathrm{i}-\mathrm{nc}-\mathrm{TiO}_{2}-\mathrm{o}$ on glass substrate as viewed from the top (a); 300-i-nc- $\mathrm{TiO}_{2}-\mathrm{o}$ free-standing domains at different magnifications showing the nanocrystal framework and the long-range ordering (b and c); and cross-sectional view of $430-\mathrm{i}-\mathrm{nc}-\mathrm{TiO}_{2}-\mathrm{o}(\mathrm{d})$. The nanocrystals with sizes ranging from 6-12 $\mathrm{nm}(\mathrm{e})$ and the crystal lattice (f) are seen at higher magnifications under STEM.

Figure 3.12. SEM images of crushed-i-nc- $\mathrm{TiO}_{2}-\mathrm{o}$ (a) and $\mathrm{nc}-\mathrm{TiO}_{2}$ (b) that were used as references.

Figure 3.13. Powder XRD (a) and Raman spectrum (b) of i-nc- $\mathrm{TiO}_{2}-\mathrm{o}$ calcined at $450{ }^{\circ} \mathrm{C}$. 
Figure 3.14. Adsorption behavior of methylene blue on $\mathrm{TiO}_{2}$ films plotted as Langmuir isotherms for nanocrystalline $\mathrm{TiO}_{2}$ (a), and inverse opals with photonic stop-band at $345 \mathrm{~nm}(\mathrm{~b}), 370 \mathrm{~nm}(\mathrm{c})$ and $500 \mathrm{~nm}(\mathrm{~d})$.

Figure 3.15. Typical extinction spectra of methylene blue during photodegradation under UV irradiation (a). Logarithmic plot of relative dye extinction (b) showing the first order decay rate for $n c-\mathrm{TiO}_{2}$ and inverse opals with stopband at 345,370 and $500 \mathrm{~nm}$.

Figure 3.16. Photo-activity enhancement factor for all $\mathrm{i}-\mathrm{nc}-\mathrm{TiO}_{2}-\mathrm{o}$ plotted as a function of stop-band energies under the same monochromatic irradiation.

Figure 3.17. Photo-activity enhancement factor for i-nc- $\mathrm{TiO}_{2}-\mathrm{o}$ with different stop-band energies, under white light irradiation $(\lambda>300 \mathrm{~nm})$ at 0 and $45 \mathrm{deg}$.

\section{CHAPTER 4}

Figure 4.1. Intrinsic defects in colloidal crystal: (a) point defects due to small spheres (black arrow); dislocation (along direction of white arrow) and stacking faults where (b) shows the normal fcc packing of $a b c$ while (c) shows stacking order of $a b a$ of a three-layered opal.

Figure 4.2. (a) Computer simulated images of guest-host colloidal crystal with $295 \mathrm{~nm}$ host spheres and guest spheres of different sizes at different concentrations (as labeled in a). (b) Schematic of donor and acceptor defect modes in a colloidal system where the dielectric material is in grey, and a plot of the defect mode frequencies as a function of defect volume. 
Figure 4.3. Transmission spectra of $(1-x) 150-\chi 180$ (a) and (1- $x) 150-x 210$ (b) polystyrene opal templates, and trends in stop-band position and the transmission difference $\left(\mathrm{T}_{\text {background }}-\mathrm{T}_{\min \text { at midgap }}\right.$ ) as a function of $x$ for $(1-x) 150-\chi 180$ opal templates (c).

Figure 4.4. The relationship between the number of (111) planes and composition in $(1-x) 150-x 180$-i-nc- $\mathrm{TiO}_{2}-\mathrm{o}$, assuming that the diffraction comes from (111) planes of closed-packing of either $150 \mathrm{~nm}$ or $180 \mathrm{~nm}$ spheres.

Figure 4.5. Reflectance spectra of $(1-x) 150-x 180-\mathrm{i}-\mathrm{nc}-\mathrm{TiO}_{2}-\mathrm{o}$ (a) and (1- $\left.x\right) 150$ x210-i-nc- $\mathrm{TiO}_{2}-\mathrm{o}$ (b) in water. Spectra are displaced vertically for clarity.

Figure 4.6. Changes in the absorbance spectra of (1-x)150- $x 180-\mathrm{i}-\mathrm{nc}-\mathrm{TiO}_{2}-\mathrm{o}$ (a) and (1-x)150- $x 180$-i-nc- $\mathrm{TiO}_{2}-\mathrm{o}(\mathrm{b})$ after subtracting the absorbance of a reference $\mathrm{TiO}_{2}$ film. Total absorbance obtained from integration plotted as a function of $x$

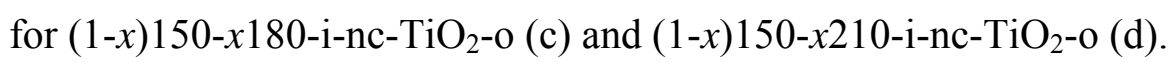

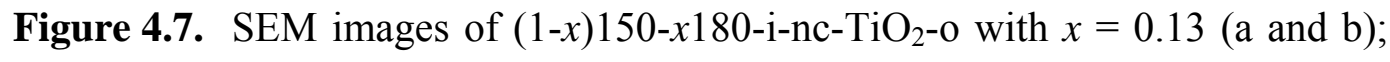
$0.37(\mathrm{c})$ and $0.57(\mathrm{~d})$.

Figure 4.8. SEM images of $(1-x) 150-x 210-\mathrm{i}-\mathrm{nc}-\mathrm{TiO}_{2}-\mathrm{o}$ with $x=0.09$ (a); 0.35 (b and c) and $0.77(d)$.

Figure 4.9. Enhancement factor of the photocatalytic efficiency of (1-x)150$x 180-\mathrm{i}-\mathrm{nc}-\mathrm{TiO}_{2}-\mathrm{o}$ and (1-x)150- $x 210-\mathrm{i}-\mathrm{nc}-\mathrm{TiO}_{2}-\mathrm{o}$ as a function of composition.

Figure 4.10. Enhancement factor for inverse structures of various binary systems with different sphere size combination $(x=0.1)$ obtained at irradiation angles normal and 45-degrees off-normal of the films.

\section{CHAPTER 5}


Figure 5.1. Theoretical enhancement factor of $\mathrm{i}-\mathrm{nc}-\mathrm{TiO}_{2}-\mathrm{o}$ in water as a function of the volume fraction of $\mathrm{TiO}_{2}$ in the inverse opal framework, as calculated by SWA.

Figure 5.2. SEM images of $\mathrm{i}$-nc- $\mathrm{TiO}_{2}-\mathrm{o}$ as-prepared (a) and after 30 minutes (b) and 45 minutes $(\mathrm{c}, \mathrm{d})$ of $\mathrm{TiF}_{6}{ }^{2-}$ treatment.

Figure 5.3. Reflectance spectra of $130-\mathrm{nm}$ templated $\mathrm{i}-\mathrm{nc}-\mathrm{TiO}_{2}-\mathrm{O}$ measured in water upon increasing the filling fraction of $\mathrm{TiO}_{2}$.

Figure 5.4. Reflectance spectra of i-nc- $\mathrm{TiO}_{2}-\mathrm{o}$ measured in water after 30 minutes of $\mathrm{TiF}_{6}{ }^{2-}$ treatment with template sphere sizes of $130 \mathrm{~nm}(\mathrm{a}), 150 \mathrm{~nm}(\mathrm{~b})$, $180 \mathrm{~nm}(\mathrm{c})$ and $210 \mathrm{~nm}(\mathrm{~d})$.

Figure 5.5. Rate of solution photocatalysis normalized with respect to the dye accessible area of various films: nc- $\mathrm{TiO}_{2}$, as-prepared $\mathrm{i}-\mathrm{nc}-\mathrm{TiO}_{2}-\mathrm{o}$ and those after increasing the $\mathrm{TiO}_{2}$ wall thickness.

\section{CHAPTER 6}

Figure 6.1. Schematic showing the pathways of the photogenerated electron and hole on a $\mathrm{TiO}_{2}$ particle whose surface is modified with Pt.

Figure 6.2. $\mathrm{SEM}$ image of $\mathrm{i}-\mathrm{Pt}-\mathrm{TiO}_{2}-\mathrm{o}(\mathrm{a})$, showing the ordered structure. STEM images of i-Pt-TiO 2 -o with $1.3 \mathrm{wt} \%$ of $\mathrm{Pt}(\mathrm{b})$, and $2.7 \mathrm{wt} \%$ of $\mathrm{Pt}(\mathrm{c})$ and (d).

Figure 6.3. Size distribution of $\mathrm{Pt}$ nanoparticles on inverse $\mathrm{TiO}_{2}$ opals obtained from HR-STEM images.

Figure 6.4. XPS spectra of $\mathrm{Pt} 4 f$ for $\mathrm{i}-\mathrm{Pt}-\mathrm{TiO}_{2}-\mathrm{o}$ with $1.3 \mathrm{wt} \% \mathrm{Pt}$ (a) and $2.7 \mathrm{wt} \%$ $\operatorname{Pt}(b)$. 
Figure 6.5. XPS spectra of the loss structure of $\mathrm{Ti} 3 p$ in $\mathrm{i}-\mathrm{TiO}_{2}-\mathrm{O}$ (a) and $\mathrm{Ti} 2 p$ in $2.7 \mathrm{wt} \% \mathrm{i}-\mathrm{Pt}-\mathrm{TiO}_{2}-\mathrm{o}(\mathrm{b})$.

Figure 6.6. Reflectance spectra of $\mathrm{i}-\mathrm{TiO}_{2}-\mathrm{o}$ and $\mathrm{i}-\mathrm{Pt}-\mathrm{TiO}_{2}-\mathrm{o}$ in water (a) and extinction spectra in air before and after Pt photodeposition (b).

Figure 6.7. Adsorption isotherm of acid orange (structure shown on the right) on $\mathrm{i}-\mathrm{TiO}_{2}-\mathrm{O}$ at $\mathrm{pH} 3$.

Figure 6.8. Photodegradation of acid orange under white-light irradiation. a) Logarithmic plot of relative dye extinction as a function of irradiation time for the blank $\left(\mathrm{SiO}_{2}\right)$, nc-TiO 2 , $\mathrm{i}-\mathrm{TiO}_{2}-\mathrm{o}$ and $\mathrm{i}-\mathrm{Pt}-\mathrm{TiO}_{2}-\mathrm{o}$ with $2.7 \mathrm{wt} \% \mathrm{Pt}$ loading. b) Firstorder decay rate constant, also shown as the enhancement factor, of i-Pt- $\mathrm{TiO}_{2}-\mathrm{O}$ with different Pt loadings in comparison to $\mathrm{nc}-\mathrm{TiO}_{2}$.

Figure 6.9. Scalar wave approximation of the theoretical amplification factor as a function of Pt loading.

\section{CHAPTER 7}

Figure 7.1. The percentage of acetone degraded by nc- $\mathrm{TiO}_{2}$ and $\mathrm{i}-\mathrm{nc}-\mathrm{TiO}_{2}-\mathrm{o}$ of different stop-band positions after $50 \mathrm{~h}$ of irradiation using a $25 \mathrm{~W}, 160 \mathrm{~V}$ incandescent light bulb.

Figure 7.2. Photographs of various types of light bulbs coated with nc- $\mathrm{TiO}_{2}$ or inc- $\mathrm{TiO}_{2}-\mathrm{O}$.

Figure 7.3. Photograph of the experimental set-up for studying the photodegradation efficiency of $\mathrm{TiO}_{2}$-coated light bulbs. 
Figure 7.4. The relative intensity of acetone (measured by GC) as a function of illumination time for the light bulbs coated with nc- $\mathrm{TiO}_{2}$ or $345-\mathrm{i}-\mathrm{nc}-\mathrm{TiO}_{2}-\mathrm{o}$, or as is (control).

Figure 7.5. SEM images of the bi-layer structure with $325-\mathrm{i}-\mathrm{nc}-\mathrm{TiO}_{2}-\mathrm{o}$ on top of 300-i-nc- $\mathrm{TiO}_{2}-\mathrm{o}$.

Figure 7.6. SEM images of the bi-layer structure comprising a thin $\mathrm{nc}-\mathrm{TiO}_{2}$ on a $\mathrm{SiO}_{2}$ opal.

Figure 7.7. SEM image of the proposed bi-layer structure consisting of 300-i-nc$\mathrm{TiO}_{2}-\mathrm{O}$ on top of a $\mathrm{SiO}_{2}$ opal. 


\section{LIST OF SCHEMES}

Scheme 2.1. Two proposed mechanisms for the photoisomerization of azobenzene - rotation of ring along the N-N double bond or via a planar transition state in which the N-N-C bond angle does not vary much (known as "inversion").

Scheme 2.2. Functionalization of $\mathrm{SiO}_{2}$ opal films with azobenzene and spacer molecule based on the reactivity of maleimide with thiol group.

\section{LIST OF TABLES}

Table 5.1. Mass and specific surface area of $\mathrm{i}-\mathrm{nc}-\mathrm{TiO}_{2}-\mathrm{o}$ before and after $\mathrm{TiF}_{6}{ }^{2-}$ treatment.

Table 6.1. Comparison of rate constants for various samples demonstrating the synergistic effect of slow photons and Pt modification. 


\title{
CHAPTER 1
}

\section{Introduction}

\begin{abstract}
A driving force in the rapidly developing field of photonic crystals has been the photonic bandgap, a range of energies where the propagation of light is completely forbidden. The photonic bandgap allows the design of photonic lattices that localize, guide and bend light at sub-micron length scales, providing opportunities for the creation of miniature optical devices and integrated optical circuits to help drive the revolution in photonics. A less well known attribute of photonic crystals is their theoretical ability to slow light to a velocity of zero. This phenomenon can be achieved at the high- and lowenergy edges of photonic stop gaps where the photonic bands are flat, and light exists as a standing wave commensurate with the photonic lattice and photons travel at a group velocity of zero, referred to as "slow photons" herein. ${ }^{[1]}$ This chapter introduces the theory of photonic crystal and slow photons, and presents how slow photons can increase the probability of absorption which scales inversely with the group velocity of light. The theoretical background lays out the requirements for optically amplifying photochemical processes using slow photons.
\end{abstract}

\subsection{Photonic Crystals}

The idea of controlling the flow of light by structuring a material into a periodic array was independently formed by John and Yablonovitch in 1987. ${ }^{[2,3]}$ These materials, 
known as photonic crystals, are ordered structures that have periodic-dielectric modulation (in one-, two- or three-dimension) in the length scale of the wavelength of light. ${ }^{[4]}$ They can alter the propagation of light and change the interaction between light and matter. For example, constructive Bragg diffraction of light by the periodic structure gives the high iridescence of opal gem stones ${ }^{[5]}$ and the color of butterfly wings ${ }^{[6-8]}$ and beetle scales $^{[9,10]}$ (Fig. 1.1).

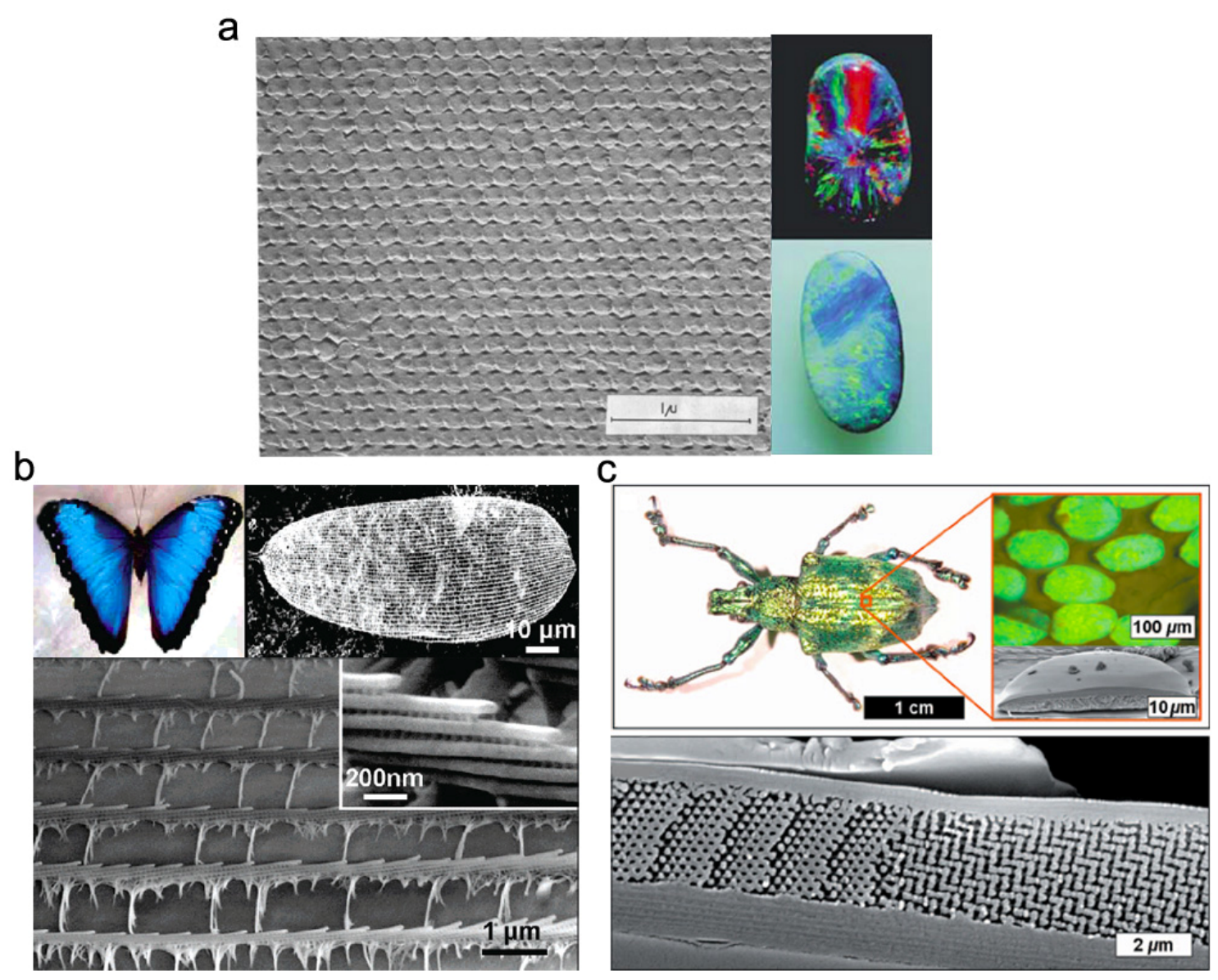

Figure 1.1. Photographs and scanning electron images showing the submicron periodic structure of natural photonic crystals: opal gem stones (a), butterfly wings (b) and beetle scales (c). Images reprinted with permission from ref. [5], [8] and [10]. Copyright International Union of Crystallography, American Chemical Society and American Physical Society. 
Aside from the natural photonic structures, the most common types of artificial photonic crystals are Bragg $\operatorname{stack}^{[11]}(1 \mathrm{D}), \operatorname{rods}^{[12]}(2 \mathrm{D})$, woodpile ${ }^{[13]}$ (3D) and colloidal crystals $^{[14]}$ also referred to as opals (3D). Their structures are shown in Fig. 1.2. Bragg stacks have long been used as distributed Bragg reflectors in waveguides and optical fibers; they consist of alternating layers of materials with different refractive index such that light is diffracted at each layer boundary to give constructive interference. They can be fabricated via top-down approach, e.g. by sputtering or evaporation of thin films, or via bottom-up techniques, such as controlled electrochemical etching of $\mathrm{Si}^{[11]}$ or spincoating of different layers of nanoparticles. ${ }^{[15]}$ Two-dimensional photonic crystal most often consists of dielectric rods in air or cylindrical holes in a material. ${ }^{[12,16]}$ Much of the earlier works on photonic crystal have focused on 2D structures owing to the ease of their
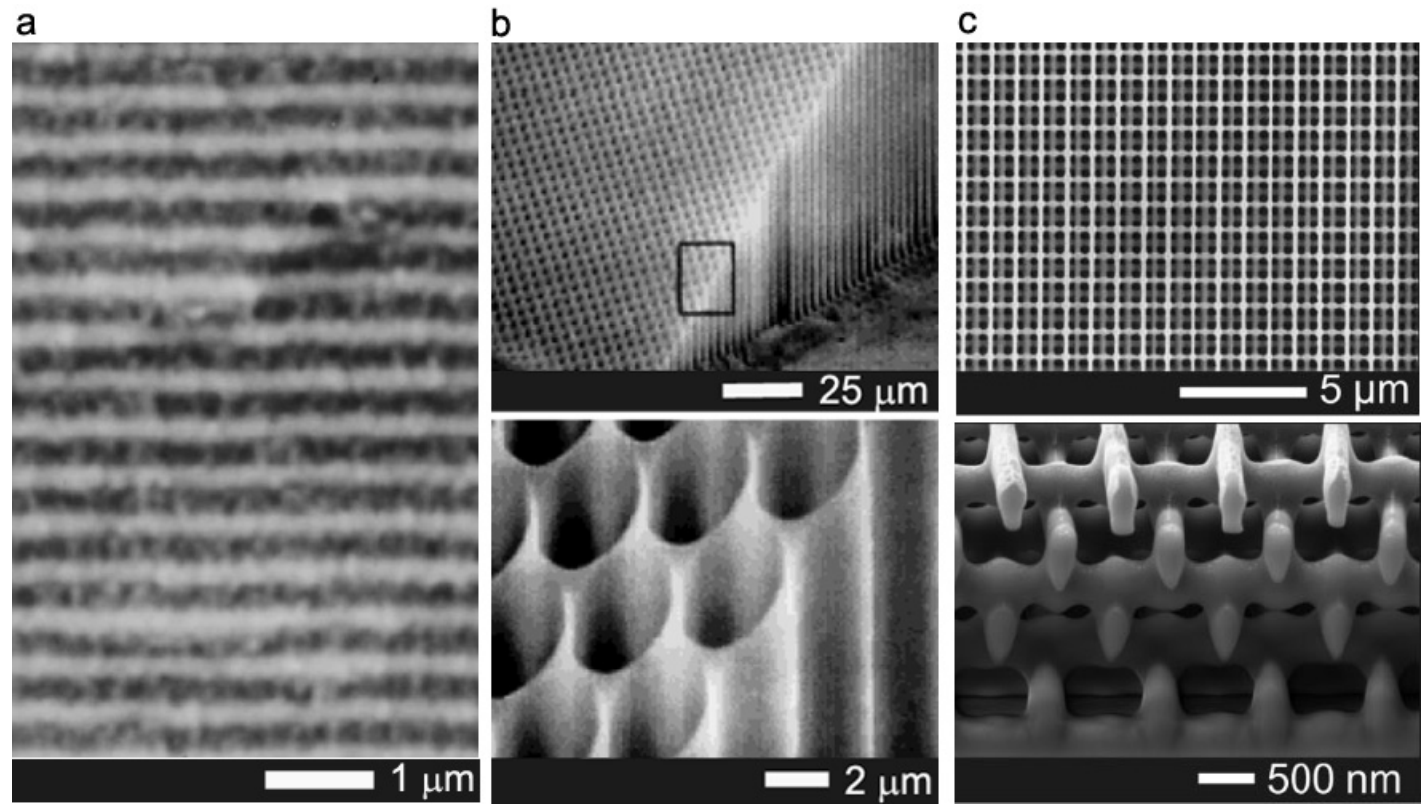

Figure 1.2. Examples of artificial one-, two- and three-dimensional photonic crystals comprised of: alternating layers of porous $\mathrm{Si}(\mathrm{a})$; cylindrical holes in $\mathrm{Si}(\mathrm{b})$ and woodpile structure of chalcogenide sulfide (c). (images reprinted with permission from ref. [11], [12] and [13]). Copyright American Institute of Physics and Wiley-VCH. 
fabrication using lithography. Because of the lack of periodicity along one axis, theoretical calculation is also more readily obtainable for $2 \mathrm{D}$ than $3 \mathrm{D}$ photonic crystals. Hence they are particularly valuable for studying wave guides, light localization at point defects $^{[17]}$ and the effect of structural disorder on photonic properties. ${ }^{[18,19]}$

\subsubsection{Three-dimensional Colloidal Photonic Crystal}

Three-dimensional photonic crystal is the subject of this thesis and therefore will be discussed in detail below. Although the physics of $3 \mathrm{D}$ photonic crystal is more complicated than that of 1D and 2D, 3D photonic crystal in the form of colloidal crystal is the most accessible photonic structure to chemists, as the building blocks are monodisperse sub-micron spheres. With the ability to synthesize highly monodisperse spheres, synthetic opals can be obtained by self-assembling the spheres into an orderedarray of cubic-closed packed colloidal crystal. The self-assembly can be induced by sedimenting the spheres or evaporating off the solvent of a solution of spheres. The former produces powders while the latter yields a single-crystal film with (111) plane parallel to the surface of a flat substrate (Fig. 1.3). ${ }^{[20,21]}$

Once an opal structure is obtained, it can be used as a template for making 3D

photonic crystals of endless materials in the form of inverse opals. ${ }^{[22-24]}$ To obtain inverse opals, first the voids of the opal are filled with material of interest, typically alkoxides for metal oxides like $\mathrm{TiO}_{2}$ and $\mathrm{ZrO}_{2}$, chemical-vapor-deposition precursors for semiconducting materials like $\mathrm{Si}$, or monomers for polymeric blends. Upon solidification of the newly introduced material, the template spheres are removed to leave behind a 3D open framework that is the inverse replica of the opal. Materials of a wide range of 
functions have been made into photonic crystals using this method, many of which respond to different stimuli such as light ${ }^{[25,26]}, \mathrm{pH}^{[27]}$, mechanical stress ${ }^{[28]}$ and electric potential ${ }^{[29]}$ or chemicals like glucose, ${ }^{[30]}$ heavy metal ions ${ }^{[31]}$ and gas molecules. ${ }^{[32]}$ Other inorganic inverse opals have been fabricated for potential applications in batteries, ${ }^{[33-35]}$ solar cells ${ }^{[36]}$ and fuel cells. ${ }^{[37]}$

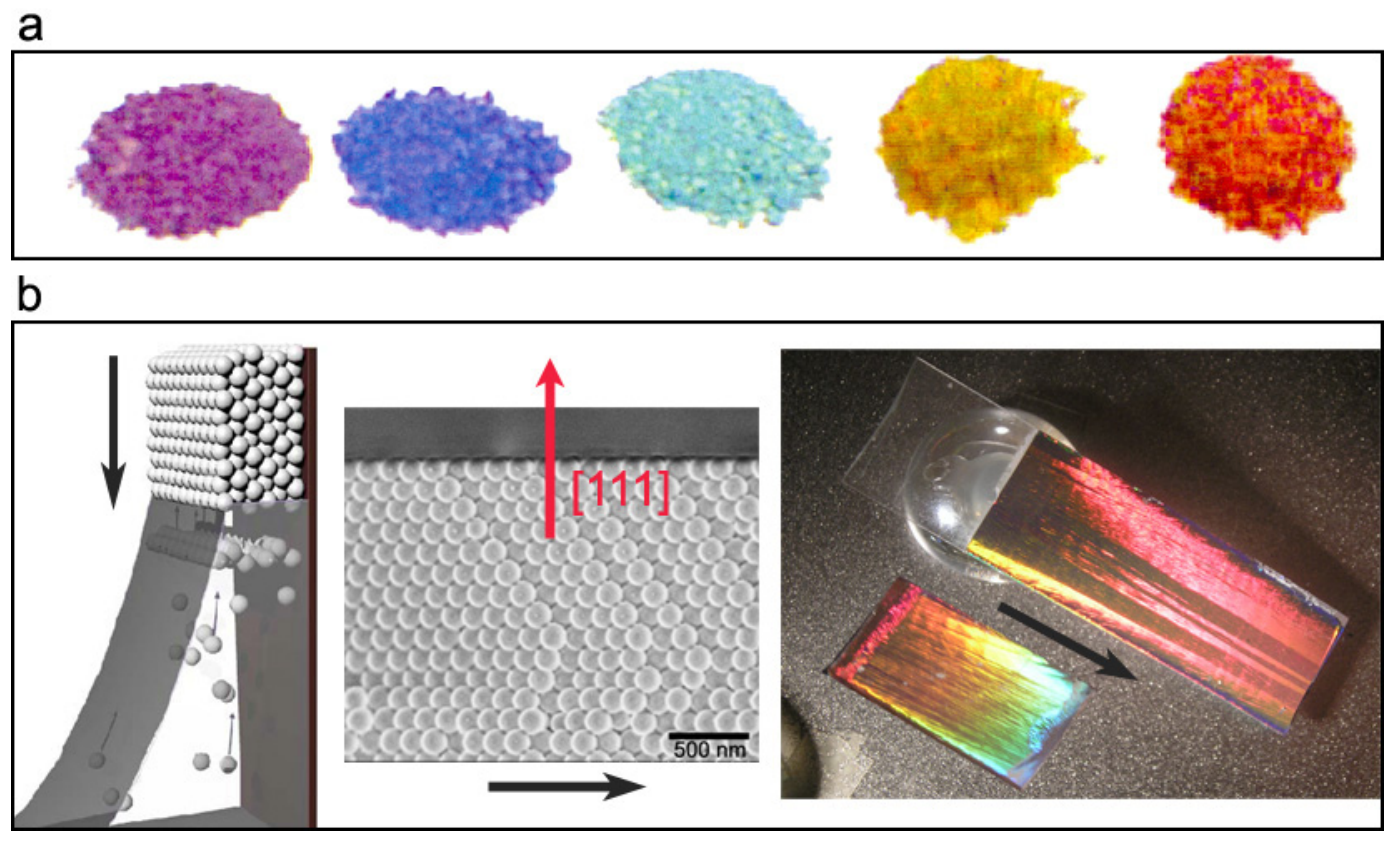

Figure 1.3. Powders of inverse colloidal crystals of various periodicities obtained from sedimentation (a) and single-crystal opal films obtained from evaporation-induced self-assembly (b). The scheme on the left in (b) shows the deposition of spheres along the meniscus of an evaporating solvent; the ordered face-centered cubic structure can be seen in the SEM image where the [111] direction is normal to the substrate; the resultant opalescence is shown in the photograph on the right. The black arrows indicate the direction of the growth of the films. (image (a) reprinted with permission from ref. [23]). Copyright American Chemical Society. 
Thin films of opal and inverse opal with sphere sizes $\sim 150 \mathrm{~nm}$ to $1 \mu \mathrm{m}$ show vivid colors, which can be explained based on a combination of Bragg's law of constructive interference in diffraction

$$
n \lambda=2 d \sin \theta
$$

and Snell's law of refraction of light

$$
\frac{n_{1}}{n_{2}}=\frac{\sin \alpha}{\sin \beta}
$$

Note that by definition the angle $\theta$ is with respect to the normal of the film, while $\alpha$ and $\beta$ are with respect to the surface of the medium. Therefore, by using cosine law to equate $\alpha$ and $\beta$ to the normal of the film, the modified Bragg's equation becomes

$$
n \lambda=2 d \sqrt{n_{e f f}^{2}-\sin ^{2} \theta}
$$

where $n$ is 1 (for fundamental stop band as explained in the following section), $\mathrm{d}$ is the (111) interplanar distance, $\theta$ the angle between incident beam and the normal of the film, and $n_{\text {eff }}$ is the effective refractive index approximated by

$$
n_{\text {eff }}=f n_{\text {sphere }}+(1-f) n_{\text {void }}
$$

with $f$ being the filling fraction of the face-centered cubic structure $(f=0.74)$. Equation 1.3 accurately determines the wavelength of the reflected light by opals and also provides a facile way to monitor the filling of the pores of the opal during the inversion process.

In addition to mimicking structural color, the artificial photonic crystals are designed to control other properties of light such as localizing the electromagnetic field or reducing the group velocity of light within the photonic crystal to enhance light-matter interactions. To better understand these phenomena, fundamental understanding of photonic band diagram is needed. 


\subsection{Theory of Photonic Band Diagram}

The photonic properties are described by a photonic band diagram, just as electronic properties are described by an electronic band diagram. Like any other macroscopic electromagnetic event, the propagation of light in photonic crystal is governed by the four macroscopic Maxwell's equations. ${ }^{[4]}$ In cgs units, they are

$$
\begin{aligned}
& \nabla \bullet B=0 \\
& \nabla \bullet D=4 \pi \rho \\
& \nabla \times E+\frac{1}{c} \frac{\partial B}{\partial t}=0 \\
& \nabla \times H-\frac{1}{c} \frac{\partial D}{\partial t}=\frac{4 \pi}{c} J
\end{aligned}
$$

where $\mathrm{c}$ is the speed of light, $\mathrm{E}$ and $\mathrm{H}$ are the electric and magnetic fields, $\mathrm{B}$ and $\mathrm{D}$ are the displacement and magnetic induction field, and $\rho$ and $\mathrm{J}$ are the free charges and currents that can be assumed as zero. In most cases, magnetic permeability is close to unity so that $\mathrm{B}=\mathrm{H}$; also, the displacement field, $\mathrm{D}(\mathrm{r})$, can be approximated as $\varepsilon(\mathrm{r}) \mathrm{E}(\mathrm{r})$ where $\varepsilon(\mathrm{r})$ is the dielectric constant. Then equations $1.5 \mathrm{a}-1.5 \mathrm{~d}$ become

$$
\begin{aligned}
& \nabla \bullet H(r, t)=0 \\
& \nabla \bullet \varepsilon(r) E(r, t)=0 \\
& \nabla \times E(r, t)+\frac{1}{c} \frac{\partial H(r, t)}{\partial t}=0 \\
& \nabla \times H(r, t)-\frac{\varepsilon(r)}{c} \frac{\partial E(r, t)}{\partial t}=0
\end{aligned}
$$

Note that the assumptions and approximations have restricted the photonic structure to be lossless and linear in optical response. Next, the fields described by Maxwell's equations 
can be written as harmonic modes that vary sinusoidally with time so that $\mathrm{H}$ and $\mathrm{E}$ can be written as

$$
\begin{aligned}
& H(r, t)=H(r) e^{i \omega t} \\
& E(r, t)=E(r) e^{i \omega t}
\end{aligned}
$$

Substituting these expressions of $\mathrm{H}$ and $\mathrm{E}$ into equations $1.6 \mathrm{a}-1.6 \mathrm{~d}$ gives

$$
\begin{aligned}
& \nabla \times E(r)+\frac{1}{c} H(r)=0 \\
& \nabla \times H(r)-\frac{i \omega}{c} \varepsilon(r) E(r)=0
\end{aligned}
$$

where $\mathrm{E}(\mathrm{r})$ can now be expressed in terms of $\mathrm{H}(\mathrm{r})$. With some rearrangement, the result is an equation that is entirely governed by $\mathrm{H}(\mathrm{r}){ }^{[38,39]}$

$$
\nabla \times\left(\frac{1}{\varepsilon(r)} \nabla \times H(r)\right)=\left(\frac{\omega}{c}\right)^{2} H(r)
$$

This master equation shows that the electromagnetism is an Eigenvalue problem where the operation on a function yields the function itself multiplied by a constant. To solve this differential equation, plane-wave expansion method based on the BlochFloquent theorem is used. The theorem suggests that the Eigen solutions of differential equations with periodic coefficients may be expressed as a product of plane waves and lattice periodic functions. The solutions obtained are plotted as a photonic band diagram; an example is shown in Fig. 1.4. The photonic band diagram summarizes the dispersion relation between the energy of light and the wave vector $\mathrm{k}$ in the reciprocal lattice. The energy is often expressed as $\omega a / 2 \pi c$ ( $a$ is the unit cell length and $\omega$ is the angular frequency) while $\mathrm{k}$ has a magnitude of $2 \pi / \lambda$ in $\hat{\mathrm{k}}$ direction within the Brillouin zone. 


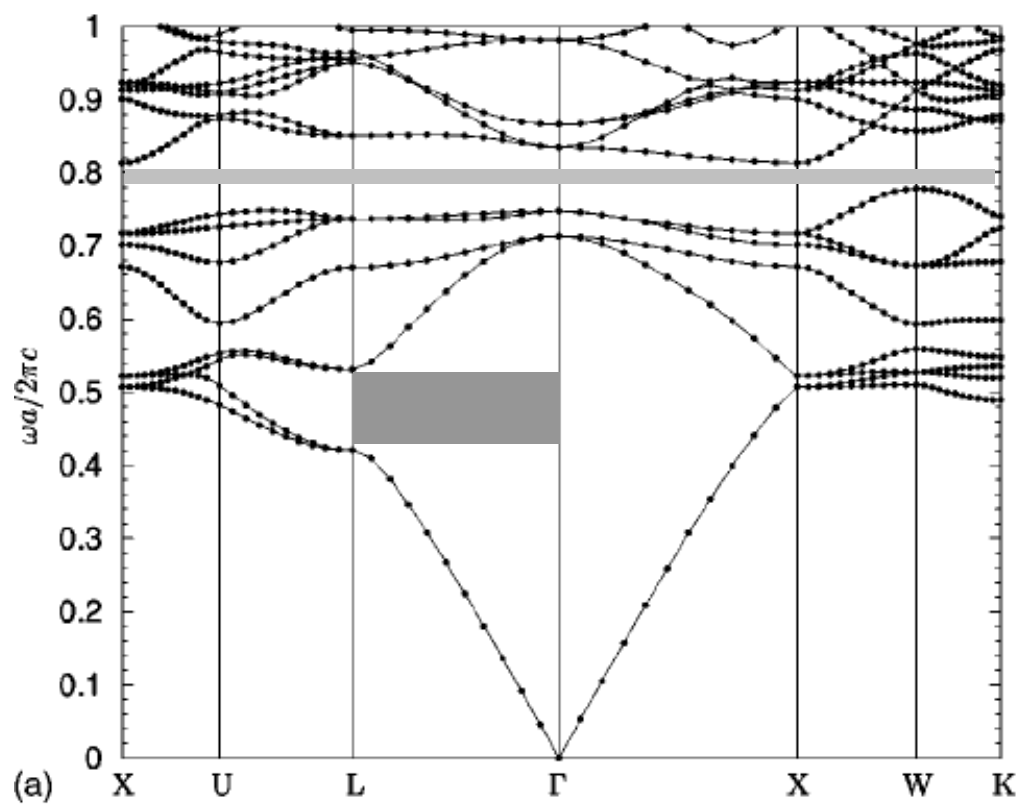

Figure 1.4. Photonic band diagram of an inverse Si opal showing the dispersion relation between energy of light and the wave vector. The light-grey shaded area indicates the complete bandgap while the dark-grey shaded area indicates one of the stop bands in this structure. (image reprinted with permission from ref. [40]). Copyright American Physical Society.

\subsubsection{Group Velocity and Density of States}

From the photonic band diagram two important properties can be derived: the group velocity of light $\left(\mathrm{vg}_{\mathrm{g}}\right)$ and the density of states (DOS). The former is defined as $\partial \omega / \partial \mathrm{k}$ (i.e. the derivative of the dispersion relation) while the latter is the integral of Bloch states of a chosen frequency over the Brillouin zone. Not only can total density of states be calculated, but local density of states at a specified region of the photonic crystal can also be calculated. ${ }^{[40]}$ As a result, information on the energy and spatial distribution of the electromagnetic field within the periodic structure can be obtained. An example of the total vs local density of states of a photonic structure for different energy of light is 
shown in Fig. 1.5. For this non-closed-packed face-centered structure, the DOS at lower energy are concentrated in the background medium while the DOS at the higher energy are localized on the dielectric spheres. The distribution of electromagnetic field can further be numerically characterized by the confinement factor, which is the ratio of local DOS over total DOS at some frequency and wave vector. ${ }^{[41]}$

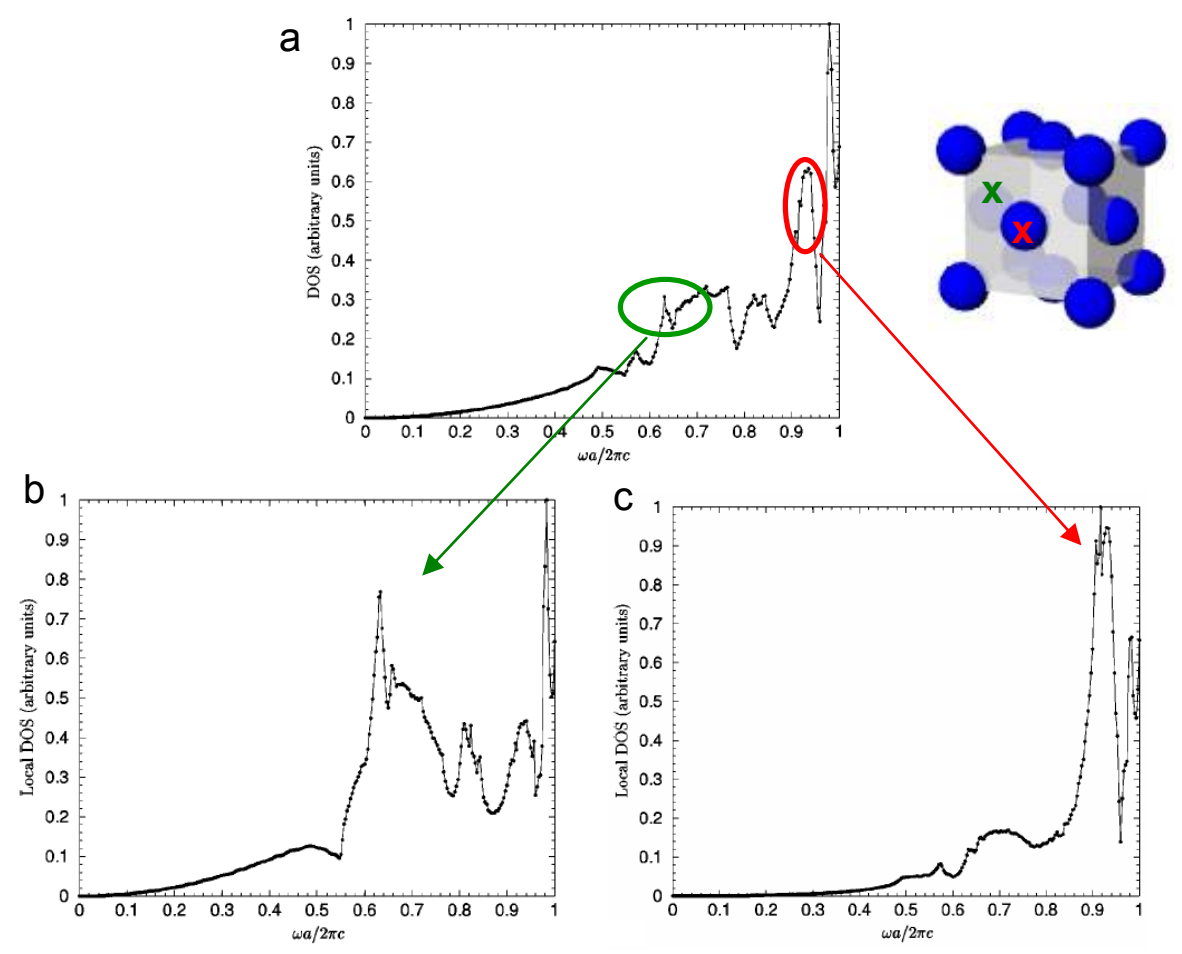

Figure 1.5. A comparison of total DOS (a) and local DOS (b and c) for a fec structure of $\mathrm{TiO}_{2}$ spheres in water. The local DOS calculated at midway between two adjacent spheres (b) and at the center of sphere (c). The filling fraction of $\mathrm{TiO}_{2}$ is $25 \%$. (images reprinted with permission from ref. [40]). Copyright American Physical Society.

When the DOS are depleted for some range of frequency, the corresponding energy of light cannot propagate through the photonic crystal. When this energy gap 
persists throughout all directions, it is referred to as the photonic bandgap; an example of such a complete bandgap is found between the $8^{\text {th }}$ and $9^{\text {th }}$ bands for an inverse Si opal ${ }^{[42]}$, as indicated by the light-grey shaded area in Fig. 1.4. On the other hand, most photonic crystals only have vanishing DOS for a specific direction of light propagation. These gaps are known as photonic stop bands, an example of which can also be found in the $\Gamma$ L Brillouin zone in Fig. 1.4 (dark-grey shaded area). In cubic-closed-packed colloidal photonic crystal, stop bands are a result of light diffraction from the (111) planes. The position of this fundamental stop band can also be calculated using Bragg's law (equation $1.3)$.

The other important property derived from the photonic band diagram is $v_{g}$, in particular the slow-photon phenomenon that arises at energies near the edges of photonic stop band. Slow photon can be perceived as a transition between full transmission of light with energy outside of the photonic band gap to the full attenuation of light with energy within the band gap. With photonic bands flattening out as Bloch states vanish near the band gap, light propagates at a reduced group velocity as $\partial \omega / \partial \mathrm{k}$ approaches 0 theoretically. This phenomenon effectively increases the optical path length of light and the interaction of light with the material. If the material is photoactive, changes in fundamental properties like absorption may occur as a result of slow photons. To better understand the impact of the reduced light group velocity on the gain and loss of the material, a dynamical treatment to the propagation of an envelope of light is required.

\subsubsection{Absorption and Non-linear Optical Properties in Photonic Crystal}


As mentioned above, the calculation of the photonic band diagram generally assumes linear, non-dispersive and lossless material. In other words, the dielectric constant of the photonic crystal is energy-independent and the absorption of light is absent. To account for any absorption or non-linearity that may arise during the propagation of an envelope of light, a dynamical treatment using Maxwell's and Schrödinger's equations was performed by Bhat and Sipe. ${ }^{[43]}$ After extensive mathematical derivation, they arrive at:

$$
\frac{\partial \Psi(s)}{\partial t}+v_{g}(\vec{k}) \frac{\partial \Psi(s)}{\partial s}=\frac{1}{2} i \frac{\partial^{2} \omega}{\partial^{2} k} \frac{\partial^{2} \Psi(s)}{\partial^{2} s}+i \omega \sigma \Psi(s)+i \frac{\alpha}{\varepsilon_{0} v_{g}(\vec{k})} \Psi(s)|\Psi(s)|^{2}
$$

for a wave packet moving in the $\hat{s}$ direction through the photonic crystal. $\Psi$ is the Schrödinger's envelope function of the field, $v_{g}$ is the group velocity as before, and $\varepsilon_{0}$ is the permittivity in vacuum. The dynamical treatment now modifies the solutions in terms of two important factors: dielectric-dispersion and non-linearity of the material. First, the effective index modification to the linear optical properties is represented by $\sigma$, which is defined as

$$
\sigma=\frac{1}{2} \int \frac{d^{3} r}{\Omega_{\text {cell }}} \frac{\varepsilon_{c}(\vec{r})}{\varepsilon_{0}}\left|E_{\alpha \vec{k}}(\vec{r})\right|^{2}
$$

where $\varepsilon_{\mathrm{c}}$ is the corrective dielectric constant that has both real and imaginary parts. Second, the non-linearity, which may arise due to localization of electromagnetic field, is taken into account by $\alpha$, also known as the effective Kerr coefficient. It again is an integral of functions in Maxwell's equations and other variables (the details of which can be found in ref. 40). 
Now suppose the field is time independent and group velocity dispersion $\left(\partial^{2} \omega / \partial k^{2}\right)$ and non-linearity are negligible, equation 1.12 simplifies to

$$
v_{g}(\vec{k}) \frac{\partial \Psi(s)}{\partial s}=i \omega \sigma \Psi(s)
$$

By writing

$$
\Psi(s)=\Psi(0) \exp [\tilde{i n}(\omega s) / c]
$$

we arrive at the following relationship

$$
\tilde{n}=\sigma\left(\frac{c}{v_{g}(\vec{k})}\right)
$$

where $\tilde{n}$ is the effective refractive index. In other words, the normal refractive index of a material upon being incorporated into a photonic structure will now be altered to $\tilde{n}$. The real part of $\tilde{n}$ is associated with changes in effective phase velocity while the imaginary part describes the effect of gain or loss on propagation. Equation 1.16 shows that $\tilde{n}$ is inversely proportional to $v_{g}$; it suggests that absorption, which is governed by the imaginary part of $\tilde{n}$, may be significantly increased for light traveling at reduced group velocity. This derivation is very important as it provides the basis for all of the work presented in this thesis.

\subsection{Scalar-wave Approximation for Modeling Photonic Properties}

Solutions to the full vectorial non-linear Schrödinger's equation require extensive computational power and time; such calculation is also not easily interpreted by scientists who are not expert in optical physics. As a result, a less rigorous modeling method Scalar Wave Approximation (SWA) - has been used to describe some of the optical 
properties of photonic crystal. ${ }^{[38,44]}$ The SWA neglects the polarization of light, the vector nature of light, thereby simplifying a three-dimensional problem into a onedimensional scalar one. The electric field for the propagation of light for each constituents of the sample is calculated ${ }^{[45]}$ :

$$
E(x)=\left\{\begin{array}{l}
\text { incident medium } \rightarrow e^{i k_{i} x}+r e^{-i k_{i} x} \\
\text { photonic crystal } \rightarrow C_{1}\left(e^{i k_{c} x}+\eta e^{i\left(k_{c}-G_{L}\right) x}\right)+C_{2}\left(e^{-i k_{c} x}+\eta e^{-i\left(k_{c}-G_{L}\right) x}\right) \\
\text { outgoing medium } \rightarrow t e^{i k_{t} x}
\end{array}\right\}
$$

where $k_{i}, k_{c}$ and $k_{t}$ indicate the wavevectors for the incident, photonic crystal and outgoing media; $G_{L}$ is the reciprocal lattice vector (e.g. $2 \pi / d_{(111)}$ for light impinging perpendicular to the surface of the colloidal photonic crystal); and $\eta$ relates to the coefficients of the field within the lattice. Other coefficients like $r, t$ and $C_{i}$ are solved by imposing the continuity of the electric field and its derivative between media. Then, an important relation between the phase difference of incident and outgoing light $(\Delta \phi)$ and group velocity of light arises:

$$
\frac{\partial \Delta \phi(\omega)}{\partial \omega}=L\left(\frac{1}{v_{g}(\omega)}-\frac{n_{t}}{c}\right)
$$

where $\mathrm{L}$ is the finite thickness of the photonic crystal.

Based on equation 1.18, the group velocity of light traveling through a colloidal crystal in [111] direction can be modeled accurately and has in fact been corroborated experimentally by measuring the phase difference of incident and outgoing light using white-light interferometry. ${ }^{[46,47]}$ Different than the calculation of the photonic band diagram, the SWA takes into account the finite thickness of the photonic crystal. Therefore SWA can model changes in $v_{g}$ with respect to the number of layers in an opal 
structure. It has been shown that significant reduction in $v_{\mathrm{g}}$ is achieved with $\sim 30$ layers of spheres in an opal, and discrepancies in $v_{\mathrm{g}}$ between theory and experiment for thicker samples are the result of light scattering due to lattice imperfections. This discrepancy can also be accounted for in SWA by introducing an effective absorption coefficient. ${ }^{[46]}$ Figure 1.6 demonstrates the close agreement between the optical spectra obtained from SWA and experiment. The $v_{g}$ of this respective sample calculated by SWA is also shown. Note that $v_{g}$ reaches a minimum right at the band-edges of the stop band. Although SWA models the fundamental stop band to high conformity as experimental optical spectra, it neglects higher-order photonic bands, which require full vectorial analysis.

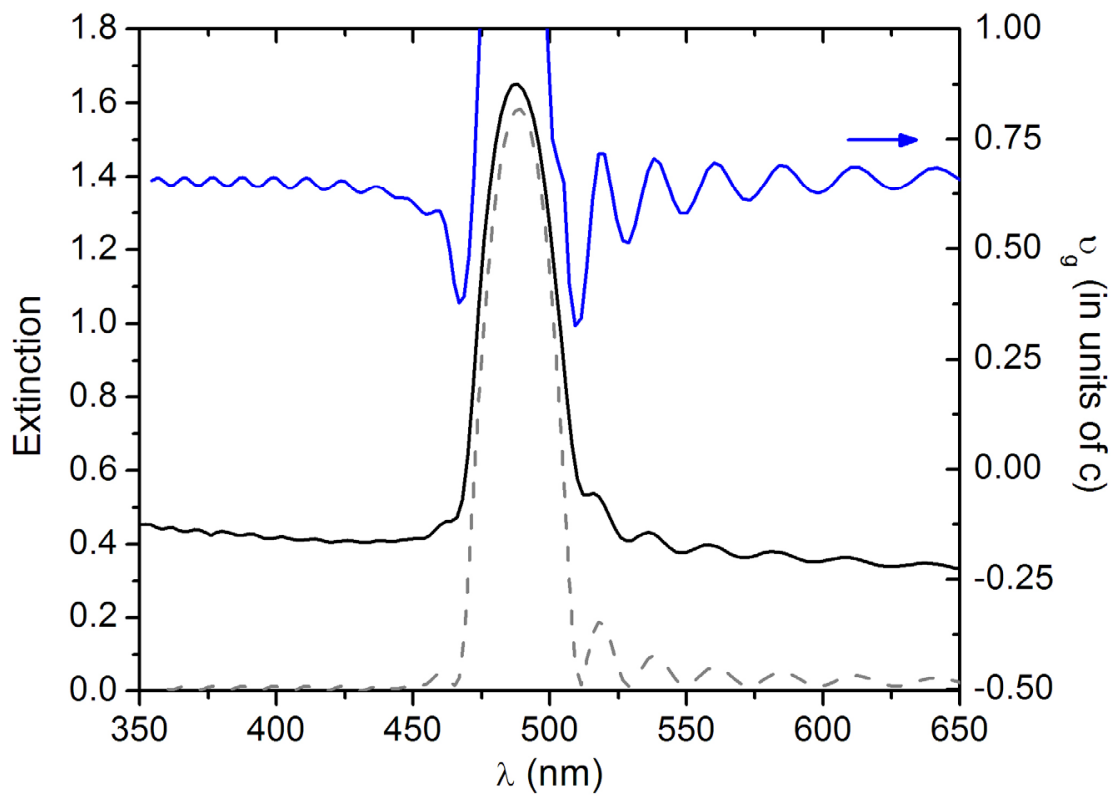

Figure 1.6. Extinction spectra of a 27-layer opal of $205 \mathrm{~nm}$ spheres measured experimentally (solid black line) vs calculated by SWA (grey dashed-line) assuming sphere size of $205 \mathrm{~nm}$, and $15 \%$ of hydration layer in the void space. The vertical offset is due to the scattering of light by defects in the opal. The group velocity, expressed as a fraction of the speed of light, is plotted above in solid blue line. 


\subsection{Slow Photons in Opal and Inverse Opal}

The concept of slow photon and light localization will now be applied to the specific structure of opal (and inverse opal). Since work reported herein only deals with the properties near the fundamental photonic stop band, those found at higher energy or other wave vector (direction) will not be addressed here. The photonic band diagram indicates a stop band in $\Gamma$-L Brillouin zone, corresponding to the [111] direction of the opal. The band immediately below the stop band is referred to as the dielectric band while that just above is the air band. The terms - dielectric and air bands - indicate where the maximum amplitude of the propagating wave exists with respect to the components of the opal. Following the dispersion relation towards the stop band, the group velocity decreases as the bands flatten out. However, for energy just below the stop band (at the red-edge of the Bragg peak), the electromagnetic field is more localized on the high dielectric part of the opal. Conversely, for energy just above the stop band (blue-edge), the field is more localized on the low dielectric part (air) of the opal. The degree of field localization depend on the contrast between the high and low dielectric material of the opal; the greater the contrast, the higher degree of localization it is on one constituent of the photonic crystal. Figure 1.7 summarizes the generalizations. In order to increase the absorption of the dielectric material, slow photons at the red-edge of the photonic stop band are sought-after. ${ }^{[1]}$ On the other hand, if the absorbing molecules reside on the interface of the dielectric material and air voids, then there should be less difference between the red- and blue-edge enhancements. 

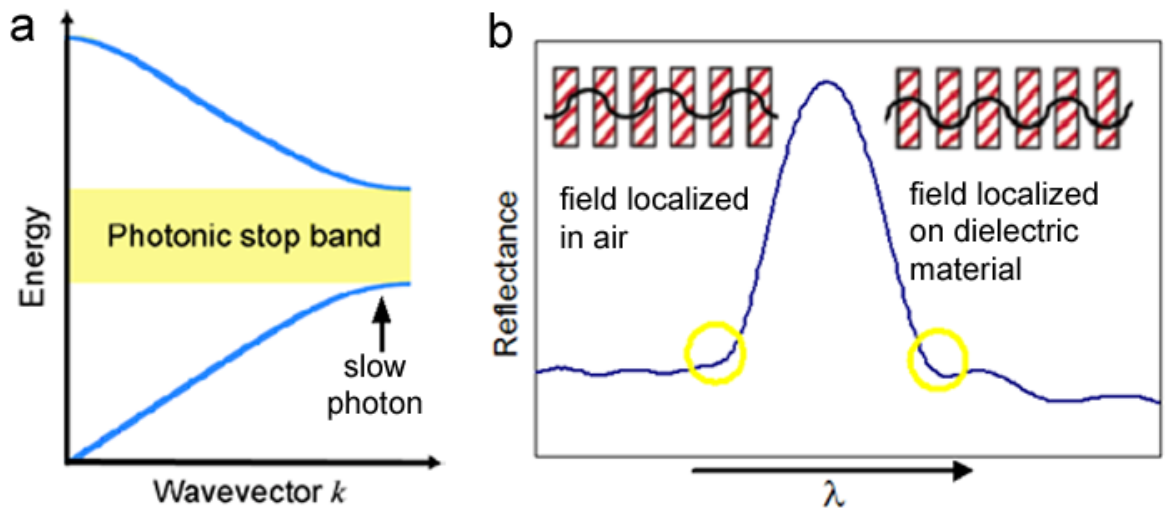

Figure 1.7. Simplified photonic band diagram of a photonic crystal (a). The position of the slow photons, indicated by the circles, with respect to the stop band reflection is shown in (b). (image adapted from ref. [36], reprinted with permission from ref. [1]). Copyright American Chemical Society and Royal Society of Chemistry.

\subsection{Examples of Photonic Band-edge Enhancement}

A significant amount of research has focused on taking advantage of the benefits of slow photons for enhancing dye absorption or emission. ${ }^{[48-50]}$ The excitation of atoms to higher atomic states by light, or the subsequent radiative relaxation, may be different due to the local coupling of photonic mode with the atomic dipole moment. ${ }^{[51]}$ Because of such altered light-matter interaction, dramatic gain-enhancements in light emitting devices and low threshold and high efficiency lasing have been demonstrated, including the simultaneous lasing of various colors in different directions of the photonic crystal ${ }^{[52]}$, and lasing at the band-edge of a cholesteric liquid crystal. ${ }^{[53,54]}$ The emission enhancement arises as a result of the reduced photon group velocity and increased photon density of states at the band edge, combined with the inhibition of emission at the photonic stop band. Slow photons have also been employed to drastically enhance the electro-optic response of lithium niobate, where an observed enhancement of 312 times at 
the band-edge of the photonic crystal suggested the possibility of micron sized nonlinear devices with very low power requirement. ${ }^{[55]}$ Originating as a fascinating optical-physics phenomenon, slow photons have now attracted scientists of a wide range of disciplines as there now exist a myriad of opportunities in enhancing photo processes and photochemical reactions.

\subsection{Recent Progress on Incorporating Photonic Structures in Photovoltaic Cells}

Various photonic structures have been incorporated with photoactive devices in recent years - the most actively pursued being the photovoltaic cells due to their importance as alternative energy resources. The pioneering work arose 5 years ago when Mallouk and co-workers, with theoretical follow-up by Mihi and Miguez, demonstrated an increased path-length of light through $\mathrm{TiO}_{2}$-based dye-sensitized solar cells by using a photonic crystal as a Bragg reflector. They obtained a $60 \%$ increase in the incidentphoton-to-current conversion efficiency. ${ }^{[36,45,56,57]}$ In another approach, O’Brien et al. achieved surface resonant modes in a thin Si film coupled to a 3D photonic crystal that diffracted light longitudinally to increase the photoconductivity by two-fold. ${ }^{[58]}$ Zeng et al. in a related study designed a two-dimensional Bragg reflector that has been shown to localize light in the active layer via total-internal reflection between the two interfaces. ${ }^{[59}$, ${ }^{60]}$ Figure 1.8 shows some of the designed architectures. Slow photons in photonic crystals present an alternative approach to increasing the path length of light, with a promising outlook in solid-state dye-sensitized solar cells ${ }^{[61]}$, thin-film based Si solar cells $^{[62]}$, hydrogen fuel generation from water, and photocatalysis - the main subject of this dissertation. 

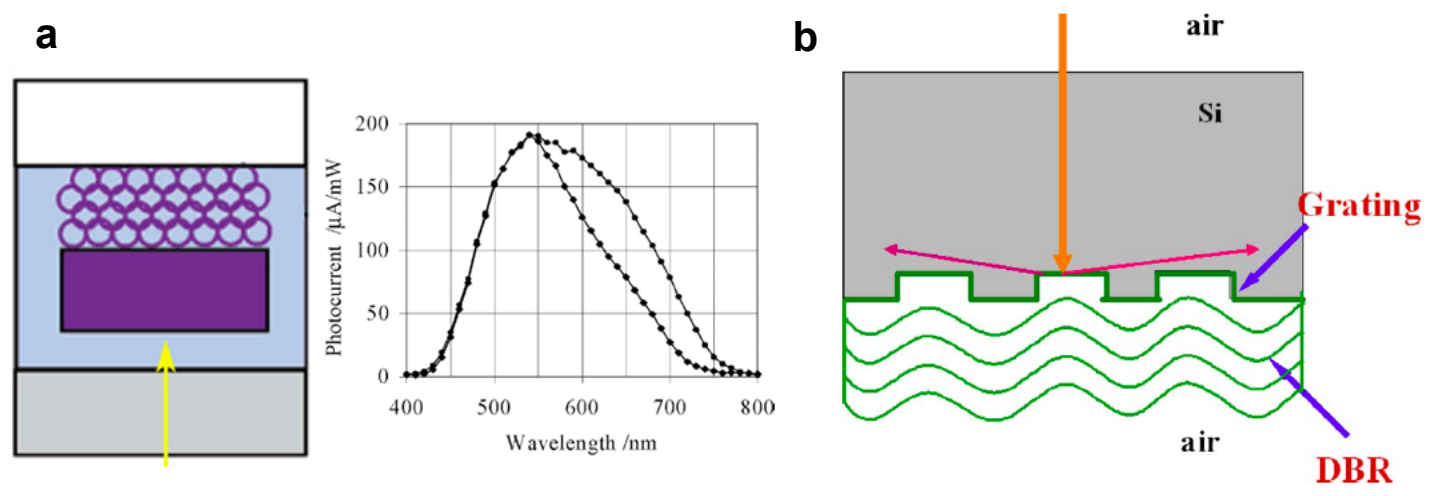

Figure 1.8. Different photonic structures for increasing the path length of light: (a) an inverse $\mathrm{TiO}_{2}$ opal on the back of a thick $\mathrm{TiO}_{2}$ absorbing layer as a wavelength-selective back reflector (an increase in the photocurrent seen on the right); and (b) a designed 2D grating with distributed Bragg reflector at the back of a silicon film. (images reprinted with permission from ref. [36] and [59]). Copyright American Chemical Society and American Institute of Physics.

\subsection{References}

[1] J. I. L. Chen, G. von Freymann, S. Y. Choi, V. Kitaev, G. A. Ozin, J. Mater. Chem. 2008, 18, 369.

[2] E. Yablonovitch, Phys. Rev. Lett. 1987, 58, 2059.

[3] S. John, Phys. Rev. Lett. 1987, 58, 2486.

[4] J. D. Joannopoulos, P. R. Villeneuve, S. Fan, Nature 1997, 386, 143.

[5] J. V. Sanders, Acta Crystallogr., Sect. A: Found. Crystallogr. 1968, 24, 427. http://journals.iucr.org/

[6] L. P. Biro, Z. Balint, K. Kertesz, Z. Vertesy, G. I. Mark, Z. E. Horvath, J. Balazs, D. Mehn, I. Kiricsi, V. Lousse, J. P. Vigneron, Phys. Rev. E 2003, 67.

[7] P. Vukusic, R. Sambles, J. Soc. Dyers Colour. 2000, 116, 376.

[8] J. Y. Huang, X. D. Wang, Z. L. Wang, Nano Lett. 2006, 6, 2325. 
[9] A. R. Parker, V. L. Welch, D. Driver, N. Martini, Nature 2003, 426, 786.

[10] J. W. Galusha, L. R. Richey, J. S. Gardner, J. N. Cha, M. H. Bartl, Phys. Rev. E 2008, 77, 050904. http://dx.doi.org/10.1103/PhysRevE.77.050904

[11] V. Agarwal, J. A. del Rio, Appl. Phys. Lett. 2003, 82, 1512.

[12] A. Birner, U. Gruning, S. Ottow, A. Schneider, F. Muller, V. Lehmann, H. Foll, U. Gosele, Phys. Status Solidi A 1998, 165, 111.

[13] S. Wong, M. Deubel, F. Perez-Willard, S. John, G. A. Ozin, M. Wegener, G. von Freymann, Adv. Mater. 2006, 18, 265.

[14] S. Wong, V. Kitaev, G. A. Ozin, J. Am. Chem. Soc. 2003, 125, 15589.

[15] S. Colodrero, M. Ocana, H. Miguez, Langmuir 2008, 24, 4430.

[16] W. M. Robertson, G. Arjavalingam, R. D. Meade, K. D. Brommer, A. M. Rappe, J. D. Joannopoulos, J. Opt. Soc. Am. B: Opt. Phys. 1993, 10, 322.

[17] E. Yablonovitch, T. J. Gmitter, R. D. Meade, A. M. Rappe, K. D. Brommer, J. D. Joannopoulos, Phys. Rev. Lett. 1991, 67, 3380.

[18] D. M. Beggs, M. A. Kaliteevski, R. A. Abram, D. Cassagne, J. P. Albert, J. Phys.: Condens. Matter 2005, 17, 1781.

[19] M. A. Kaliteevski, J. Manzanares Martinez, D. Cassagne, J. P. Albert, Phys. Rev. $B$ 2002, 66, 1131011.

[20] P. Jiang, J. F. Bertone, K. S. Hwang, V. L. Colvin, Chem. Mater. 1999, 11, 2132.

[21] D. M. Mittleman, J. F. Bertone, P. Jiang, K. S. Hwang, V. L. Colvin, J. Chem. Phys. 1999, 111, 345.

[22] J. E. G. J. Wijnhoven, W. L. Vos, Science 1998, 281, 802. 
[23] R. C. Schroden, M. Al-Daous, C. F. Blanford, A. Stein, Chem. Mater. 2002, 14, 3305.

[24] A. Stein, R. C. Schroden, Curr. Opin. Solid State Mater. Sci. 2001, 5, 553.

[25] M. K. Maurer, I. K. Lednev, S. A. Asher, Adv. Func. Mater. 2005, 15, 1401.

[26] M. Kamenjicki, I. K. Lednev, A. Mikhonin, R. Kesavamoorthy, S. A. Asher, Adv. Func. Mater. 2003, 13, 774.

[27] K. Lee, S. A. Asher, J. Am. Chem. Soc. 2000, 122, 9534.

[28] A. C. Arsenault, T. J. Clark, G. von Freymann, L. Cademartiri, R. Sapienza, J. Bertolotti, E. Vekris, S. Wong, V. Kitaev, I. Manners, R. Z. Wang, S. John, D. Wiersma, G. A. Ozin, Nature Mater. 2006, 5, 179.

[29] A. C. Arsenault, D. P. Puzzo, I. Manners, G. A. Ozin, Nature Photonics 2007, 1, 468.

[30] M. Ben-Moshe, V. L. Alexeev, S. A. Asher, Anal. Chem. 2006, 78, 5149.

[31] A. V. Goponenko, S. A. Asher, J. Am. Chem. Soc. 2005, 127, 10753.

[32] K. W. Kimble, J. P. Walker, D. N. Finegold, S. A. Asher, Anal. Bioanal. Chem. 2006, 385, 678 .

[33] N. S. Ergang, J. C. Lytle, K. T. Lee, S. M. Oh, W. H. Smyrl, A. Stein, Adv. Mater. 2006, $18,1750$.

[34] K. T. Lee, J. C. Lytle, N. S. Ergang, S. M. Oh, A. Stein, Adv. Func. Mater. 2005, $15,547$.

[35] J. Lee, W. Choi, J. Phys. Chem. B 2005, 109, 7399.

[36] S. Nishimura, N. Abrams, B. A. Lewis, L. I. Halaoui, T. E. Mallouk, K. D. Benkstein, J. Van de Lagemaat, A. J. Frank, J. Am. Chem. Soc. 2003, 125, 6306. 
[37] G. A. Umeda, W. C. Chueh, L. Noailles, S. M. Haile, B. S. Dunn, Energy Environ. Sci. 2008, 1, 484.

[38] S. Satpathy, Z. Zhang, M. R. Salehpour, Phys. Rev. Lett. 1990, 64, 1239.

[39] K. M. Ho, C. T. Chan, C. M. Soukoulis, Phys. Rev. Lett. 1990, 65, 3152.

[40] K. Busch, S. John, Phys. Rev. E 1998, 58, 3896.

http://dx.doi.org/10.1103/PhysRevE.58.3896

[41] R. D. Meade, A. M. Rappe, K. D. Brommer, J. D. Joannopoulus, J. Opt. Soc. Am. B: Opt. Phys. 1993, 10, 328.

[42] A. Blanco, E. Chomski, S. Grabtchak, M. Ibisate, S. John, S. W. Leonard, C. Lopez, F. Meseguer, H. Miguez, J. P. Mondia, G. A. Ozin, O. Toader, H. M. van Driel, Nature 2000, 405, 437.

[43] N. A. R. Bhat, J. E. Sipe, Phys. Rev. E 2001, 64, 056604.

[44] K. W. K. Shung, Y. C. Tsai, Phys. Rev. B 1993, 48, 11265.

[45] A. Mihi, H. Miguez, J. Phys. Chem. B 2005, 109, 15968.

[46] G. von Freymann, S. John, S. Wong, V. Kitaev, G. A. Ozin, Appl. Phys. Lett. 2005, $86,053108$.

[47] J. F. Galisteo-Lopez, M. Galli, M. Patrini, A. Balestreri, L. C. Andreani, C. Lopez, Phys. Rev. B 2006, 73, 125103.

[48] L. Bechger, P. Lodahl, W. L. Vos, J. Phys. Chem. B 2005, 109, 9980.

[49] K. Yoshino, S. B. Lee, S. Tatsuhara, Y. Kawagishi, M. Ozaki, A. A. Zakhidov, Appl. Phys. Lett. 1998, 73, 3506.

[50] K. Yoshino, S. Tatsuhara, Y. Kawagishi, M. Ozaki, A. A. Zakhidov, Z. V. Vardeny, Appl. Phys. Lett. 1999, 74, 1648. 
[51] S. John, J. Wang, Phys. Rev. Lett. 1990, 64, 2418.

[52] M. N. Shkunov, Z. V. Vardeny, M. C. DeLong, R. C. Polson, A. A. Zakhidov, R. H. Baughman, Adv. Func. Mater. 2002, 12, 21.

[53] W. Cao, A. Munoz, P. Palffy-Muhoray, B. Taheri, Nature Mater. 2002, 1, 111.

[54] Y. Matsuhisa, Y. Huang, Y. Zhou, S. Wu, R. Ozaki, Y. Takao, A. Fujii, M. Ozaki, Appl. Phys. Lett. 2007, 90, 091114.

[55] M. Roussey, M. P. Bernal, N. Courjal, D. V. Labeke, F. I. Baida, R. Salut, Appl. Phys. Lett. 2006, 89, 241110.

[56] L. I. Halaoui, N. M. Abrams, T. E. Mallouk, J. Phys. Chem. B 2005, 109, 6334.

[57] A. Mihi, M. E. Calvo, J. A. Anta, H. Miguez, J. Phys. Chem. C 2008, 112, 13.

[58] P. G. O'Brien, N. P. Kherani, S. Zukotynski, G. A. Ozin, E. Vekris, N. Tetreault, A. Chutinan, S. John, A. Mihi, H. Miguez, Adv. Mater. 2007, 19, 4177.

[59] L. Zeng, Y. Yi, C. Hong, J. Liu, N. Feng, X. Duan, L. C. Kimerling, B. A. Alamariu, Appl. Phys. Lett. 2006, 89, 111111.

[60] P. Bermel, C. Luo, L. Zeng, L. C. Kimerling, J. D. Joannopoulos, Opt. Express 2007, 15, 16986.

[61] P. R. Somani, C. Dionigi, M. Murgia, D. Palles, P. Nozar, G. Ruani, Sol. Energy Mater. 2005, 87, 513.

[62] T. Suezaki, P. G. O'Brien, J. I. L. Chen, E. Loso, N. P. Kherani, G. A. Ozin, Adv. Mater. 2009, 21, 559. 


\title{
CHAPTER 2
}

\section{Tracing the Effect of Slow Photon in Photoisomerization of Azobenzene}

(Reproduced in part with permission from Adv. Mater. 2008, 20, 4784. Copyright 2008

Wiley-VCH Verlag GmbH \& Co. KGaA.)

\begin{abstract}
Theory predicted that slow photons at the band-edges of the photonic stop band can increase the probability of absorption of a photoactive material. Subsequently, the increased absorption may increase the rate of the photoactivated process. To investigate this hypothesis, the photoisomerization of azobenzene anchored on silica opal (azo- $\mathrm{SiO}_{2}$ o) was studied. Here, the effect of photonic crystal properties on the rate of trans-cis photoisomerization was examined, and the enhancement arising from slow photons was explicitly traced out with respect to the band-edges of the photonic stop band. Fundamental aspect of the effect of thickness on the optical enhancement, a critical factor for applications, was further investigated. The finding indeed supports previous measurements on the group velocity of light traversing through an opal. The work demonstrates the versatility of slow photons for enhancing the photochemistry of organic-based systems. It provides insight into the fundamental understanding of slow photons and a blueprint for designing optically enhanced photochemical systems.
\end{abstract}




\subsection{Introduction}

Since the first observation by Hartley ${ }^{[1]}$, the mechanism, kinetics and theory of photoisomerization of azobenzene have been investigated in detail $\left.{ }^{[2,} 3\right]$ making azobenzene the reactive group of choice for many photochemical applications. They include: the photocontrolable solutions that turn from clear to cloudy due to isomerization-induced aggregation of colloids or formation of micelles ${ }^{[4-7]}$; optical actuators that change dimension or thickness in response to light to produce either mechanical work ${ }^{[8,9]}$ or optical signals ${ }^{[10]}$; photoresponsive ion transport membrane ${ }^{[11,12]}$; and photoregulation of biomolecules such as $\mathrm{DNA}^{[13]}$ and peptides ${ }^{[14,15]}$ (Fig. 2.1). The function of all of the systems relies on the isomerization of the thermodynamically more stable trans-azobenzene to the cis-form under irradiation of UV light. Upon trans to cis photoisomerization, the distance between para-positions of the two benzene rings decreases from $1.0 \mathrm{~nm}$ to $0.59 \mathrm{~nm}$, the dipole moment increases from 0 to ca. 3 Debye ${ }^{[16]}$ and the absorption spectrum changes considerably. The back reaction (cis-trans) occurs thermally or can be accelerated by visible light, typically with wavelength of ca. $450 \mathrm{~nm}$. Figure 2.2 shows the absorbance spectra of the isomers of azobenzene. Although the absorption spectra between the two isomers are quite different, there is no energy range that one isomer absorbs exclusively. Hence the overall rate of photoisomerization is governed by the difference in kinetics between the forward and backward reactions. With regard to the photoisomerization mechanism, some suggested that the trans-cis isomerization proceeds via rotation while the cis-trans occurs via an inversion route ${ }^{[1,3,17]}$, as shown in scheme 2.1; though this proposal remains an active debate. 


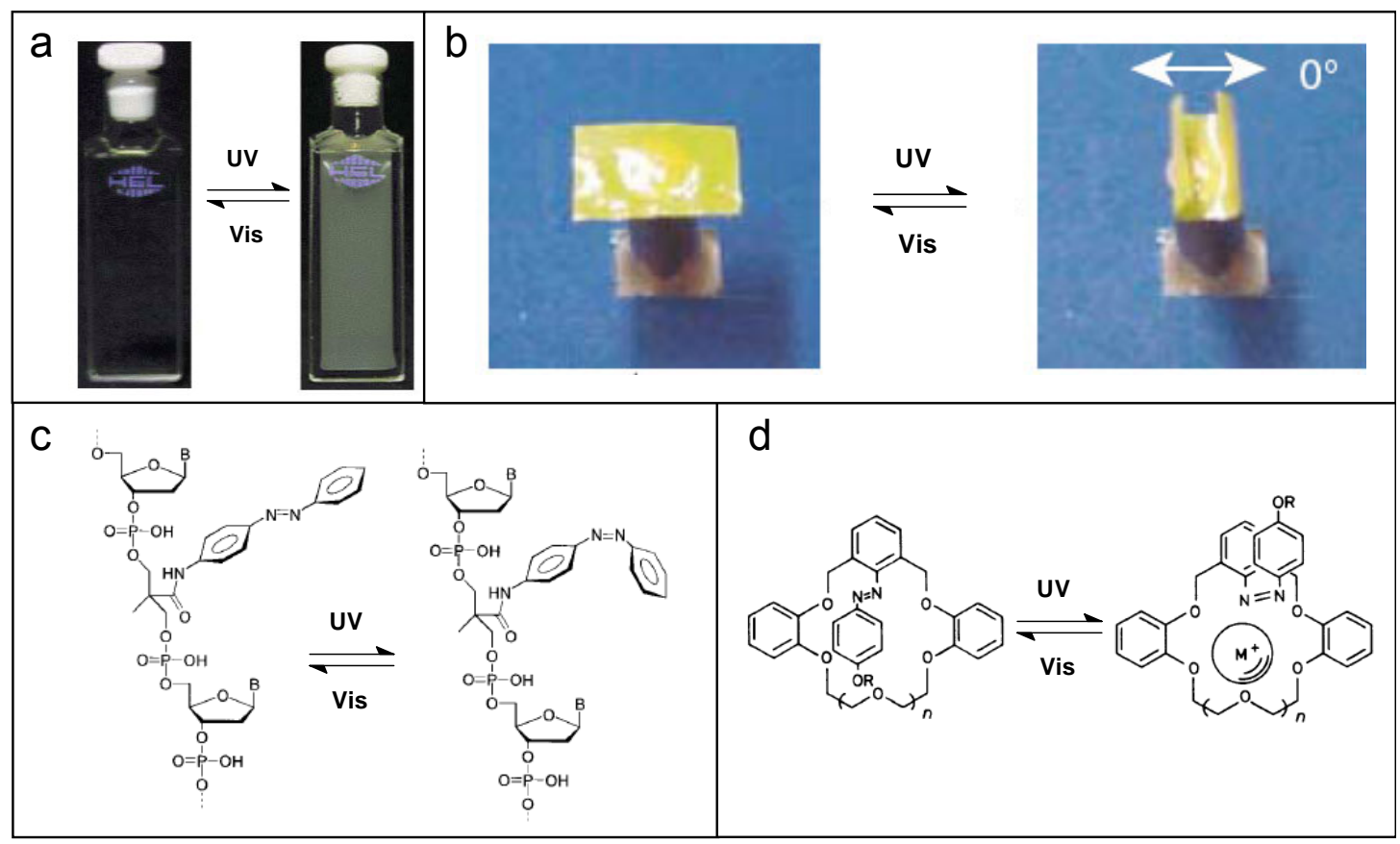

Figure 2.1. Examples of applications of azobenzene: (a) photoinduced microemulsion of heptane and water using azobenzene derived surfactant; (b) direct bending of a liquid-crystal polymer film containing azobenzene moiety under uni-directional irradiation; (c) photoisomerization of azobenzene-incorporated DNA for controlled duplex formation and (d) photoresponsive iontransport membrane. (images reprinted with permission from ref. [6], [8] and [13]). Copyright American Chemical Society, Macmillan Publishers and Wiley-VCH.

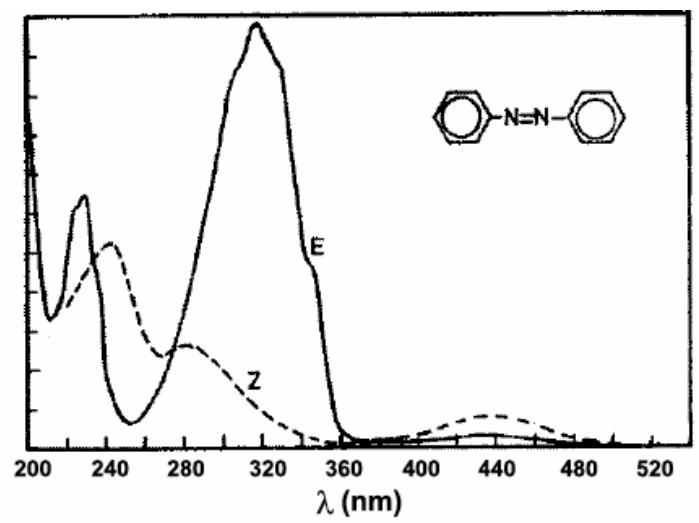

Figure 2.2. Absorption spectra of trans- (E) and cis- (Z) azobenzene in ethanol. (image reprinted with permission from ref. [2]). Copyright Elsevier Science. 
Scheme 2.1. Two proposed mechanisms for the photoisomerization of azobenzene - rotation of ring along the $\mathrm{N}-\mathrm{N}$ double bond or via a planar transition state in which the N-N-C bond angle does not vary much (known as “inversion”).

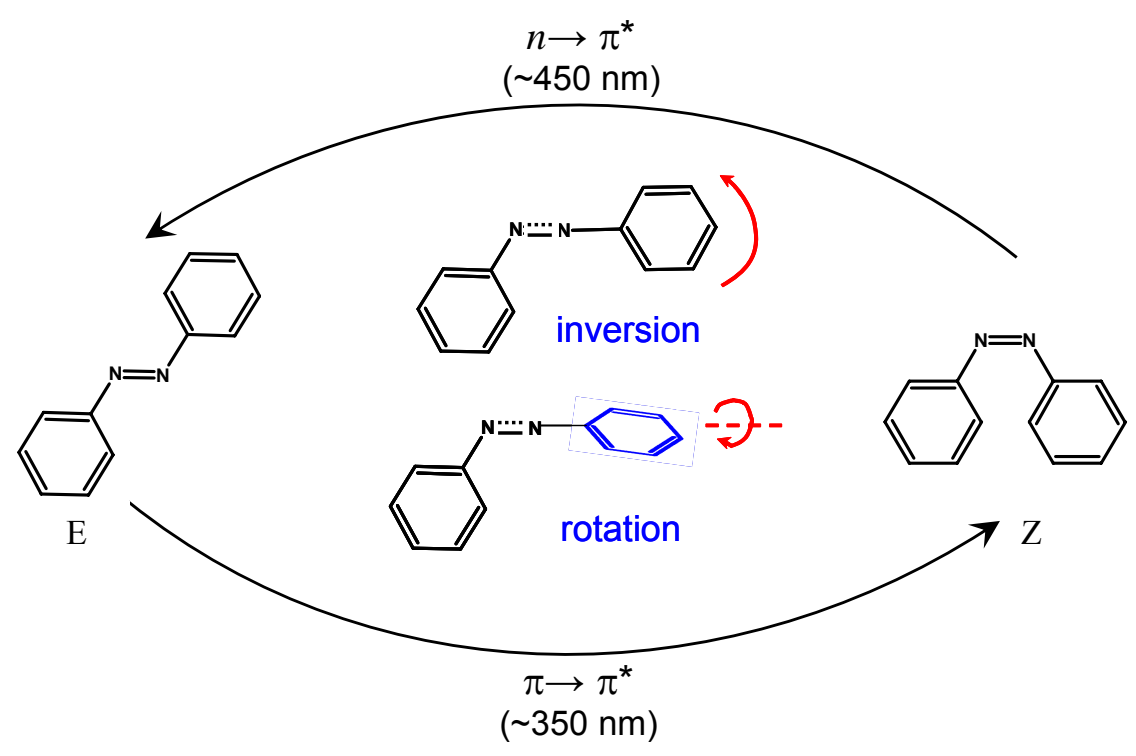

The kinetics of photoisomerization (neglecting thermal back reaction) is described by $^{[2]}$

$$
\ln \frac{\mathrm{A}_{\infty}^{\xi}-\mathrm{A}^{\xi}}{\mathrm{A}_{\infty}^{\xi}-\mathrm{A}_{0}^{\xi}}=-1000 \mathrm{I}_{0}^{\lambda}\left(\varepsilon_{\mathrm{E}}^{\lambda} \phi_{\mathrm{E}}^{\lambda}+\varepsilon_{\mathrm{Z}}^{\lambda} \phi_{\mathrm{Z}}^{\lambda}\right) \int \frac{1-10^{-\mathrm{A}^{\lambda}(\mathrm{t})}}{\mathrm{A}^{\lambda}(\mathrm{t})} \mathrm{dt}
$$

where $\mathrm{A}$ is the absorbance either at irradiation wavelength $\lambda$ or monitoring wavelength $\xi$ (note that $\mathrm{A}_{\infty}$ is the absorbance at photostationary state), $\mathrm{I}_{0}{ }^{\lambda}$ is the irradiation intensity, $\phi$ is the quantum yield of photoisomerization $\left(\phi_{\mathrm{E}}\right.$ for $\mathrm{E} \rightarrow \mathrm{Z}$ and $\phi_{\mathrm{Z}}$ for $\mathrm{Z} \rightarrow \mathrm{E}$ ) and $\varepsilon$ is the absorption coefficient for each isomer at the irradiation wavelength. The fraction $\frac{1-10^{-A^{*}(t)}}{A^{\lambda}(t)}$ is referred as the photokinetic factor, which takes into account the changes in the number of photons absorbed over time. Rearranging eq. 2.1 gives 


$$
\mathrm{A}^{\xi}=\mathrm{A}_{\infty}^{\xi}+\left(\mathrm{A}_{0}^{\xi}-\mathrm{A}_{\infty}^{\xi}\right) \exp \left(-k \int \frac{1-10^{-\mathrm{A}^{\lambda}(\mathrm{t})}}{\mathrm{A}^{\lambda}(\mathrm{t})} \mathrm{dt}\right)
$$

where

$$
k=1000 \mathrm{I}_{0}^{\lambda}\left(\varepsilon_{\mathrm{E}}^{\lambda} \phi_{\mathrm{E}}^{\lambda}+\varepsilon_{\mathrm{Z}}^{\lambda} \phi_{\mathrm{Z}}^{\lambda}\right)
$$

The first-order rate constant, $k$, can therefore be obtained by plotting the absorbance as a function of the time integral of the photokinetic factor.

For supported self-assembled azobenzene monolayer, factors that affect the isomerization, such as chain length, functional groups and spacer molecules, have been well studied in the last decade, particularly on gold substrates. ${ }^{[18,19]}$ Azobenzene groups have also been incorporated into photonic crystals for applications such as photoswitchable wettability ${ }^{[20]}$ and optical signals. ${ }^{[21]}$ In the latter, Asher and co-workers explored photochemically tunable photonic crystal consisting of azobenzenefunctionalized hydrogel and $\mathrm{SiO}_{2}$ spheres. While they demonstrated tuning of the photonic stop band by application of light that lead to the swelling of the hydrogel, there was no interplay of photonic properties and the absorption of azobenzene. By anchoring azobenzene molecules directly on $\mathrm{SiO}_{2}$ opals, the present study looks at the effects of the optical properties of the photonic crystal on the kinetics of the photoisomerization. The aim of this work is to investigate such a relationship and demonstrate the feasibility of slow photons at enhancing the rate of reaction due to the longer path length of light.

\subsection{Experimental Methods}

\subsubsection{Fabrication of Azobenzene-functionalized $\mathrm{SiO}_{2}$ Opal}


Silica opal films $\left(\mathrm{SiO}_{2}-\mathrm{o}\right)$ of sphere sizes 85,145 and $175 \mathrm{~nm}$ were obtained using the vertical deposition method of EISA. ${ }^{[22]}$ Briefly, a cleaned glass substrate was placed vertically in a vial containing ethanolic solution of the spheres. The solvent was evaporated over $\sim 3$ days at $35{ }^{\circ} \mathrm{C}$, during which time silica spheres deposited on the substrate. The thickness was controlled by adjusting the concentration of the sphere solutions. The azobenzene group was chemically anchored by reacting 4phenylazomaleinanil with thiol groups that have been functionalized on the $\mathrm{SiO}_{2}$ opal ( $\left.\mathrm{SH}-\mathrm{SiO}_{2}-\mathrm{o}\right)$. Briefly, the $\mathrm{SiO}_{2}-\mathrm{o}$ was exposed to air plasma for 5 minutes in order to increase the number of hydroxyl groups prior to being immersed in a hexane solution containing 1 vol\% of (3-mercaptopropyl)-trimethoxysilane (MPTES) for 2 hours. The film was rinsed with anhydrous ethanol and dried under nitrogen. Successful silanization was judged by the increase in the contact angle of water on the film. The $\mathrm{SH}-\mathrm{SiO}_{2}-\mathrm{O}$ was then immersed in a DMSO solution of $7 \mathrm{mM}$ 4-phenylazomaleianil overnight to yield azobenzene-functionalized silica opal $\left(\mathrm{azo}-\mathrm{SiO}_{2}-\mathrm{o}\right)$. For experiments involving the spacer, a mixture of 4-phenylazomaleianil and $N$-methylmaleimide was used with the total concentration of molecules unchanged $(7 \mathrm{mM})$. All chemicals were purchased from Aldrich.

\subsubsection{Photoisomerization Experiment}

The photoisomerization of azo- $\mathrm{SiO}_{2}-\mathrm{o}$ was performed using a Xe lamp (60 - 120 W) in combination with an Oriel monochromator. The beam was $\sim 1 \mathrm{~cm} \times 2 \mathrm{~mm}$ in irradiation area. Extinction spectra of the films over time under irradiation wavelengths ranging from 320 to $370 \mathrm{~nm}$ were measured using a Perkin Elmer Lambda 900 UV-Vis- 
NIR spectrometer; the kinetics of photoisomerization was obtained by measuring the extinction of the film at $330 \mathrm{~nm}$. Upon reaching the photostationary state, the azo- $\mathrm{SiO}_{2}-\mathrm{O}$ was switched back using light of $450 \mathrm{~nm}$. The films can be reused without noticeable change in the reaction rate.

To compare the kinetics of photoisomerization of different films, the rate constant $k$ was determined according to equation 2.2 using Microcal Origin as the curve-fitting software. The errors in $k$ were obtained from the fitting, which had R-squares over 0.995 for all data. The rate constants of azo- $\mathrm{SiO}_{2}-\mathrm{o}$ of 145 and $175 \mathrm{~nm}$ spheres were divided by that of the reference $\mathrm{SiO}_{2}$ film ( $85 \mathrm{~nm}$ spheres) under each irradiation wavelength to give the enhancement factor (EF).

\subsection{Photoisomerization of Azobenzene Anchored on $\mathrm{SiO}_{2} \mathrm{Opal}$}

Azobenzene was anchored on silica opals with sphere diameters of 85, 145 and $175 \mathrm{~nm}$. Scheme 2.2 shows the process of functionalization; the extinction spectra after each step are shown in Fig. 2.3. A small red-shift of $\sim 2 \mathrm{~nm}$ in the stop band position is

Scheme 2.2. Functionalization of $\mathrm{SiO}_{2}$ opal films with azobenzene and spacer molecule based on the reactivity of maleimide with thiol group.

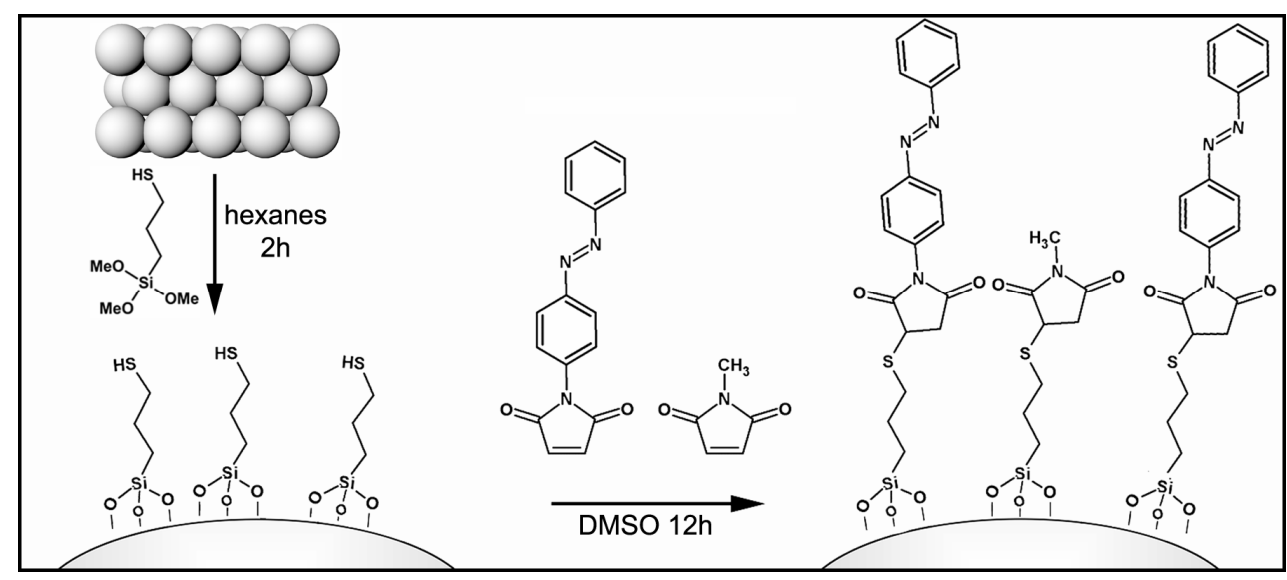



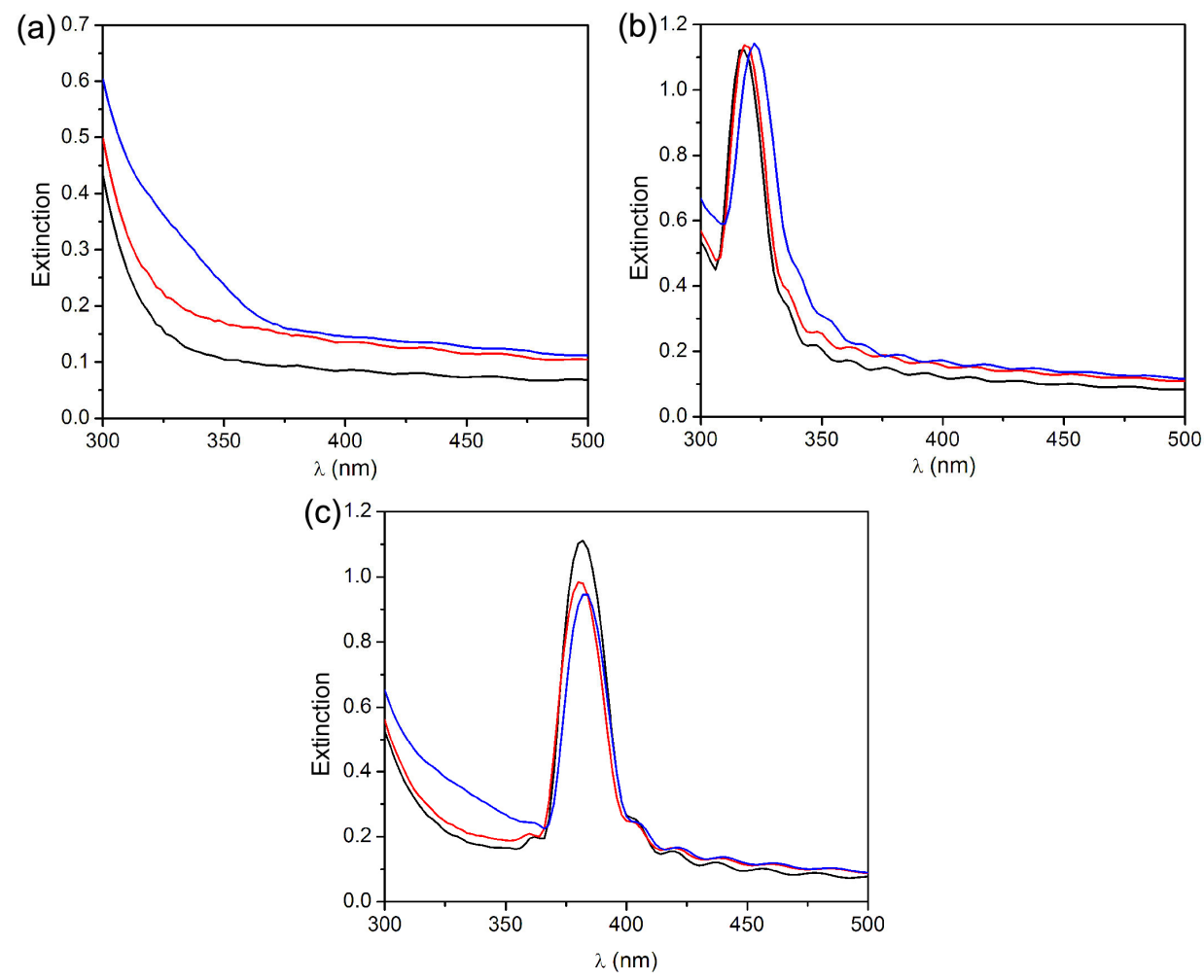

Figure 2.3. Extinction spectra of reference $\mathrm{SiO}_{2}-\mathrm{o}$ of $85 \mathrm{~nm}$ sphere size (a), $318-\mathrm{SiO}_{2}-\mathrm{o}$ (b) and $380-\mathrm{SiO}_{2}$-o (c) prior to functionalization (black), after reacting with MPTES (red) and with 4phenylazomaleinanil (blue). A shift in the stop band position was observed after surface functionalization.

observed after functionalization. From Fig. 2.3, the increase in absorption arising from trans-azobenzene (300-360 nm) is clearly evident upon functionalization. Moreover, $\mathrm{SiO}_{2}-\mathrm{O}$ with $85 \mathrm{~nm}$ spheres has no photonic stop band in the observable ultraviolet-visible range due to the small size of the silica spheres, effectively serving as the reference sample. The opals with 145 and $175 \mathrm{~nm}$ spheres have photonic stop bands at 318 and 380 $\mathrm{nm}$, respectively (herein they are denoted as 318- and 380 -azo- $\mathrm{SiO}_{2}-\mathrm{o}$, according to their stop band position). Their positions were strategically chosen to be just red and blue of 
the peak absorption of trans-azobenzene; hence slow photons at both the red- and blueedge of the stop bands can be investigated.

The amount of azobenzene anchored on the opal can be controlled by changing the concentration of MPTES, and all samples were treated with 1 vol\% of MPTES. This concentration was chosen according to Fig. 2.4, which shows the amount of azobenzene chemically bonded to $\mathrm{SiO}_{2}-\mathrm{O}$ as a function of the concentration of MPTES. At $1-5 \mathrm{vol} \%$ of MPTES, the surface coverage of azobenzene remains constant - all of the hydroxyl groups on silica have reacted to form a monolayer of azobenzene. Higher concentration (e.g. 10 vol\%) often lead to excess surface deposition during the rinsing and drying steps, also evident by the increase in light scattering of the film. By choosing monolayer coverage (using 1 vol\% MPTES), the spatial density of azobenzene on $\mathrm{SiO}_{2}$-o with different sphere sizes should be comparable and therefore not a factor in the comparison between samples.

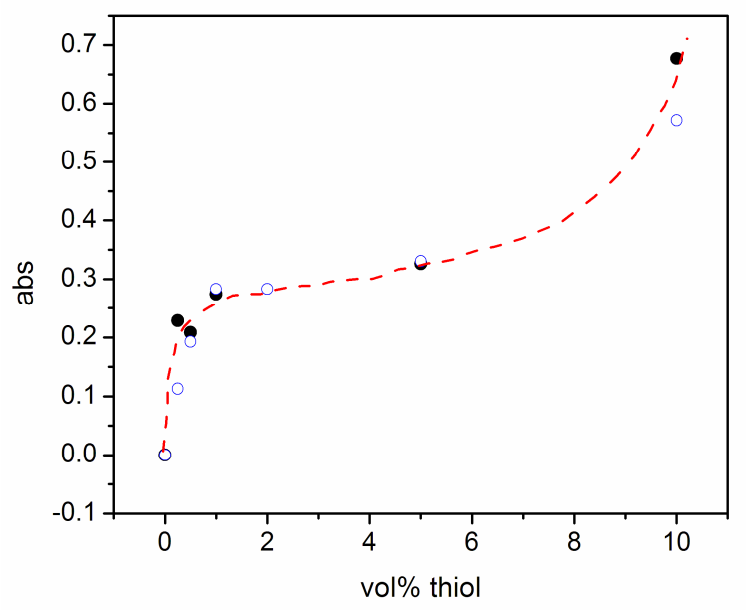

Figure 2.4. Absorbance of $380-\mathrm{azo}_{-} \mathrm{SiO}_{2}$-o (open) and reference azo- $\mathrm{SiO}_{2}$-o (filled) films after background subtraction with respect to the concentration of (3-mercaptopropyl)-trimethoxysilane. The dashed line is a guide to the eye. 
As-functionalized trans-azo- $\mathrm{SiO}_{2}-\mathrm{O}$ was photoisomerized to the cis form by irradiating with monochromatic UV light (wavelengths ranging from 320 to $370 \mathrm{~nm}$ ). Representative spectra that outline the evolution of the absorption over irradiation time are shown in Fig. 2.5. In all cases, the $\pi$ to $\pi^{*}$ absorption peak $(325 \mathrm{~nm})$ decreases in intensity while the $\mathrm{n}$ to $\pi^{*}$ transition (450 $\mathrm{nm}$ ) arising from the cis isomer becomes more apparent. Note that the absorbance profile of azobenzene is altered by the photonic properties in 318- and 380-azo- $\mathrm{SiO}_{2}-\mathrm{o}$; a strong dip is observed at the wavelengths of the stop band.
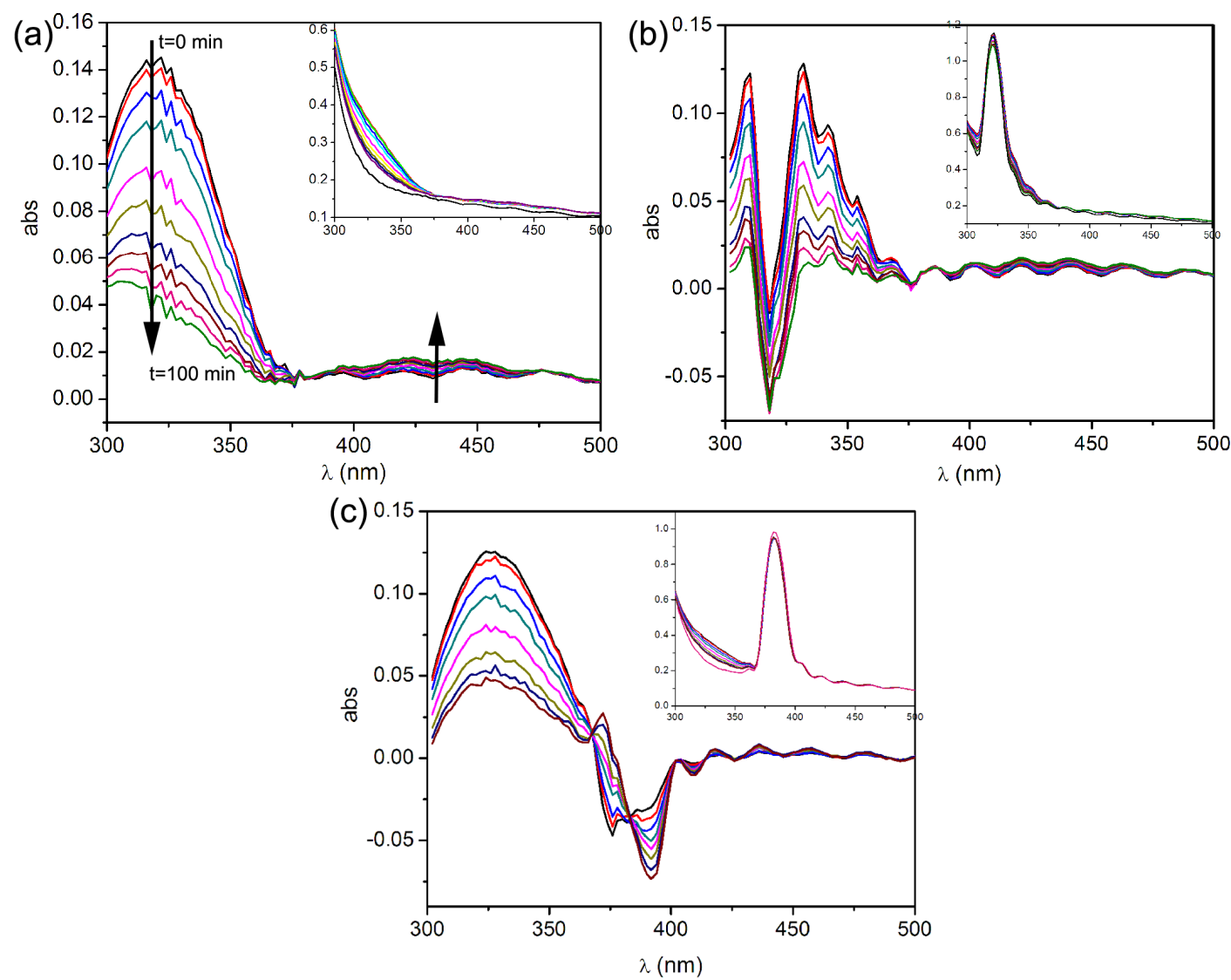

Figure 2.5. Evolution of the absorbance spectra of azo- $\mathrm{SiO}_{2}-\mathrm{o}$ of $85 \mathrm{~nm}$ (a), 318-azo- $\mathrm{SiO}_{2}-\mathrm{o}$ (b) and $380-\mathrm{azo}_{-} \mathrm{SiO}_{2}$-o (c) under UV-irradiation after background subtraction (inset shows the extinction of the films). 
According to equation 2.2, the kinetics of photoisomerization follows a first-order rate law with respect to the integral of the photokinetic factor over time, and this relationship can be established by measuring the changes in the absorbance of azobenzene upon irradiation. Figure 2.6 shows such a correlation from which the rate constant, $k$, of trans-cis photoisomerization for each sample at different irradiation wavelengths is derived. The rate constant contains information about $\mathrm{I}_{0}, \phi_{\mathrm{E}}, \phi_{\mathrm{Z}}, \varepsilon_{\mathrm{E}}$ and $\varepsilon_{\mathrm{Z}}$. Given that the irradiation intensity and quantum yields, which depend on the chemical nature of the molecules, are the same in all cases, any change in the rate constant reflects changes in the absorption coefficients. In the following section, wavelength-dependent $k$ for 318- and 380-azo- $\mathrm{SiO}_{2}-\mathrm{o}$ is investigated and for the first time the changes in the absorption coefficient of the photoactive moiety is indirectly traced out.

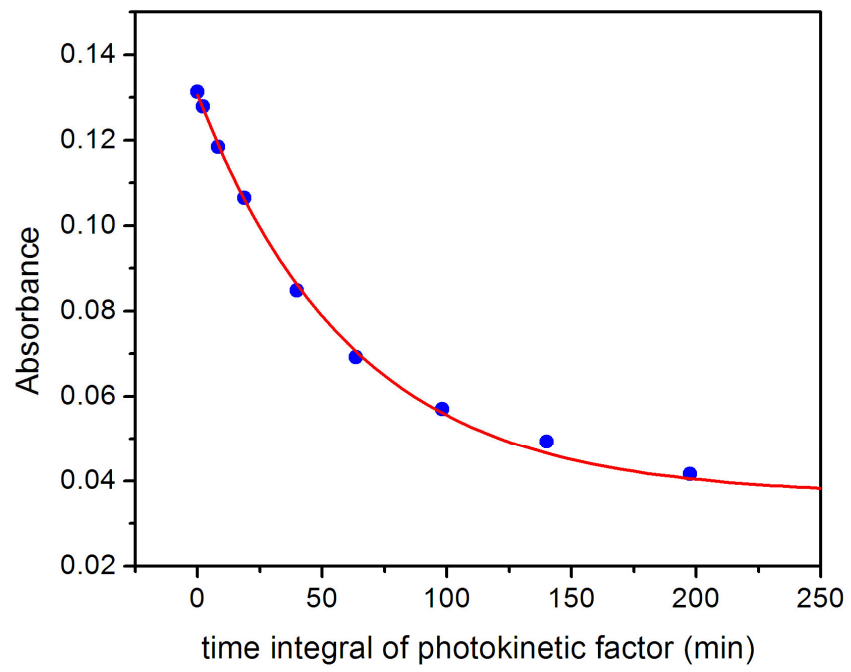

Figure 2.6. Absorbance of azo- $\mathrm{SiO}_{2}-\mathrm{o}$ at $330 \mathrm{~nm}$ as a function of $\int \frac{1-10^{-\mathrm{A}^{\lambda}(\mathrm{t})}}{\mathrm{A}^{\lambda}(\mathrm{t})} \mathrm{dt}$. A first-order exponential decay rate law was used to fit the data to obtain rate constants (see equation 2.2). 


\subsubsection{Effect of Slow Photons and Photonic Stop Band}

To study quantitatively the effect of slow photons on the rate of photoisomerization, the rate constants of 318 - and 380 -azo- $\mathrm{SiO}_{2}$-o were divided by that of the reference sample (azo- $\mathrm{SiO}_{2}$-o with $85 \mathrm{~nm}$ spheres) to give the value of the enhancement factor (EF). The wavelength-dependent EF for 318- and 380-azo-SiO shown in Fig. 2.7; the optical spectra are also plotted for easy reference of EF to the position of the photonic stop band. Figure 2.7 shows that the EF peaks at $340 \mathrm{~nm}$ near the red-edge of 318-azo- $\mathrm{SiO}_{2}-\mathrm{o}$. Similarly, the EF peaks at $355 \mathrm{~nm}$ near the blue-edge of 380-azo-SiO 2 -opal. The enhancements can be attributed to slow photons that can increase the effective optical path length of light thereby enhancing the interaction of light with matter. Since azobenzene is anchored on $\mathrm{SiO}_{2}$ spheres and is at the interface of the high $\left(\mathrm{SiO}_{2}\right)$ and low (air) dielectric media, slow photons at both the blue-edge (with electromagnetic field more localized in air) and the red-edge (with electromagnetic field more localized on $\mathrm{SiO}_{2}$ ) show an enhancement effect. As the refractive index contrast between $\mathrm{SiO}_{2}$ and air is not large the highest EF achieved was 1.35, consistent with the theoretical SWA calculations for films of 27 layers in thickness. However, the enhancement for 318-azo-SiO 2 -o is slightly lower than expected, which may be due to the electronic band edge absorption of $\mathrm{SiO}_{2}$ that begins in this range. Some researchers have also reported that structural disorder can lead to a higher amount of diffuse light scattering at the low-energy edge than the high-energy edge. ${ }^{[23,24]}$ These two factors may contribute to the lower-than-expected red-edge enhancement in $318-\mathrm{azo}_{-} \mathrm{SiO}_{2}-\mathrm{o}$. In addition to the slow-photon enhancement, Fig. 2.7 shows that the EF decreases to below 1 for both 318 - and 380 -azo- $\mathrm{SiO}_{2}$-o as the irradiation wavelength approaches the center 
of the photonic stop band. Because the photonic stop band reflects light strongly, light of this energy cannot propagate through the film and the rate of photoisomerization becomes lower than the reference film. By mapping out the wavelength-dependent $k$, the effects of slow photons and stop band on the kinetics of photoisomerization are elucidated.

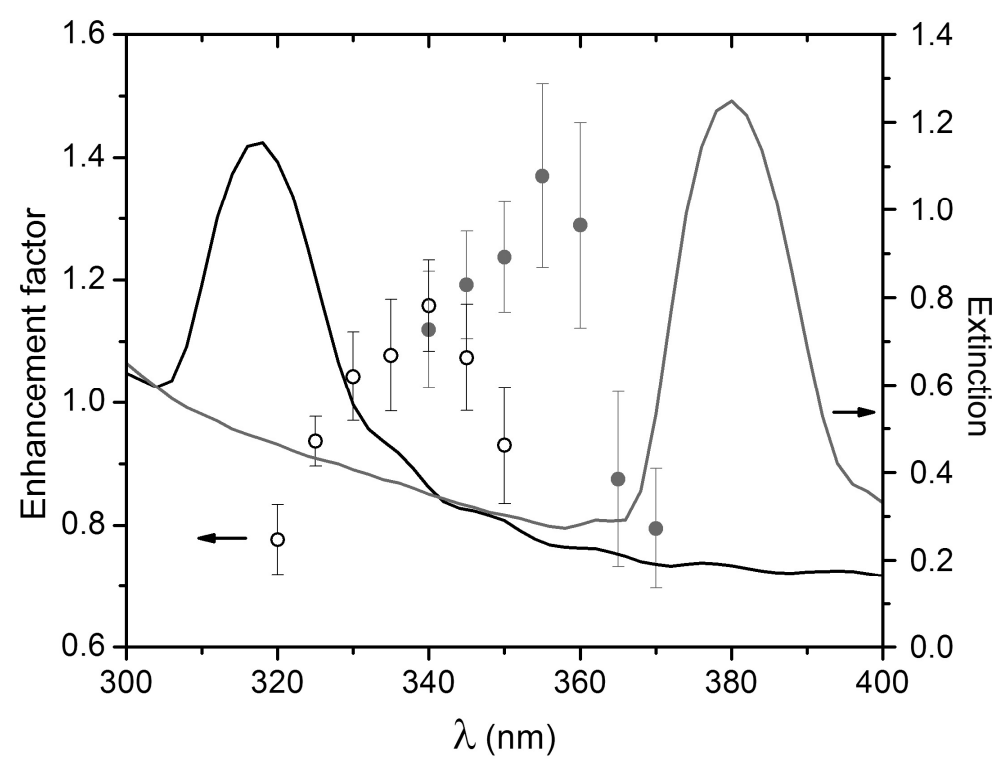

Figure 2.7. Wavelength-dependent enhancement factor for the trans-cis photoisomerization of 318-azo- $\mathrm{SiO}_{2}$-o and 380-azo- $\mathrm{SiO}_{2}$-o (open and solid circles respectively). The extinction spectra of the corresponding azo- $\mathrm{SiO}_{2}-\mathrm{O}$ are shown as references for comparing EF with respect to the position of the photonic stop band.

\subsubsection{Effect of Thickness of Opal}

Previous measurements have revealed that the finite thickness of the opal film governs the degree of reduction in the group velocity of light near the edges of the stop band. Using white-light interferometry, von Freymann et al. and Lopez et al. measured the group velocity of slow light with respect to the number of layers of spheres and found 
that group velocity decreases with increasing number of layers up to 30. The group velocity then reaches a limit as light scattering becomes more dominant with increasing thickness. ${ }^{[23,24]}$ Figure 2.8 shows the group delay (a measure of the transit time of light through a material) of light traveling through silica opals of 7,13 and 19 layers as measured by interferometry with theoretical calculation from SWA. Notably, as group delay increases at both the red- and blue-edge of the photonic stop band with increasing number of layers up to $\sim 20$ layers, there exists a greater discrepancy between theory and

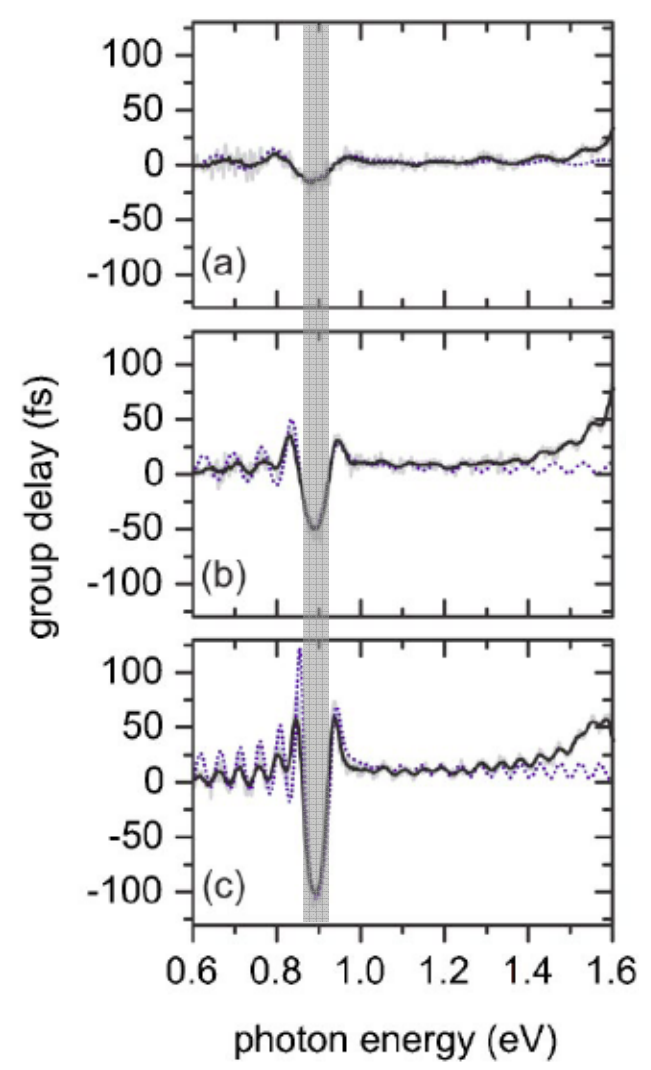

Figure 2.8. Group delay for opals with 7 (a), 13 (b) and 19 (c) layers. Increased group delay, in other words reduced group velocity, is observed at the edges of the photonic stop band (stop band position indicated by the shaded area). The experimental data is smoothed and shown as black lines while theoretical calculation is shown as dotted lines. (image reprinted with permission from ref. [24]). Copyright American Institute of Physics. 
experiment for the low energy edge. This discrepancy may be due to the higher diffuse scattering intensity at the red-edge ${ }^{[25]}$, where a small change in $\mathrm{k}$ for wavelengths within the stop band can lead to propagating states in dielectric bands of other Brillouin zones. ${ }^{[26]}$ As a result of the increased diffuse scattering at the red-edge, the enhancement at the blue-edge is higher than at the red-edge, as discussed previously.

To investigate the thickness-dependence of the rate of photoisomerization, 380azo- $\mathrm{SiO}_{2}$-o with thicknesses varying from 9 to 50 layers were irradiated with light of wavelength near the blue-edge of the stop band $(355 \mathrm{~nm})$ where slow photons are found according to Fig. 2.7. Figure 2.9 shows that EF increases almost linearly with increasing number of layers from 9 to 27 , and then it stays constant at $1.3-1.35$ for thicknesses up to 50 layers. A large deviation of experimental EF from theoretical prediction starts at $\sim 30$ layers in agreement with previous studies that suggest the onset of significant amount of light scattering at this thickness. However, if scattering prevented light from propagating through the whole thickness of the film, then the constant EF above 30 layers might be due to an averaging effect of azobenzenes that isomerized at the top of the film and those that did not isomerize at the bottom of the film. It is therefore important to examine the amount of azobenzene that photoisomerized, proportional to the change in absorbance at photostationary state (PSS), with respect to film thickness. As shown in Fig. 2.10, the amount of photoisomerizable azobenzene increases with increasing number of layers without saturation. The slope of the linear fit suggests that for each layer of spheres, azobenzene molecules equivalent to an absorbance of 0.0026 isomerized under irradiation. Based on the changes in absorbance at PSS, a complete isomerization of azobenzene molecules was achieved in all samples. As a result, it can be concluded that 
light still propagated through the entire film and the scattering of light indeed affects the group velocity for samples thicker than 30 layers. This finding further provides indirect support to the trend in group velocity measured previously.

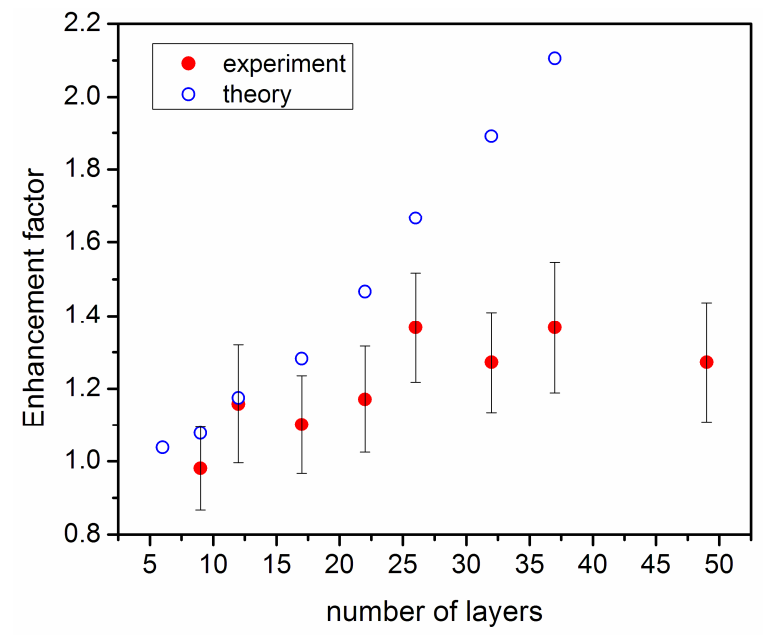

Figure 2.9. Enhancement factor for $380-\mathrm{azo}_{-} \mathrm{SiO}_{2}$-o of various thicknesses under $355 \mathrm{~nm}$ irradiation in comparison to a reference azo- $\mathrm{SiO}_{2}-\mathrm{o}$. Theoretical EF was calculated based on the SWA as the ratio of group velocity of light through a film of same effective refractive index to that of the $\mathrm{SiO}_{2}$ opal.

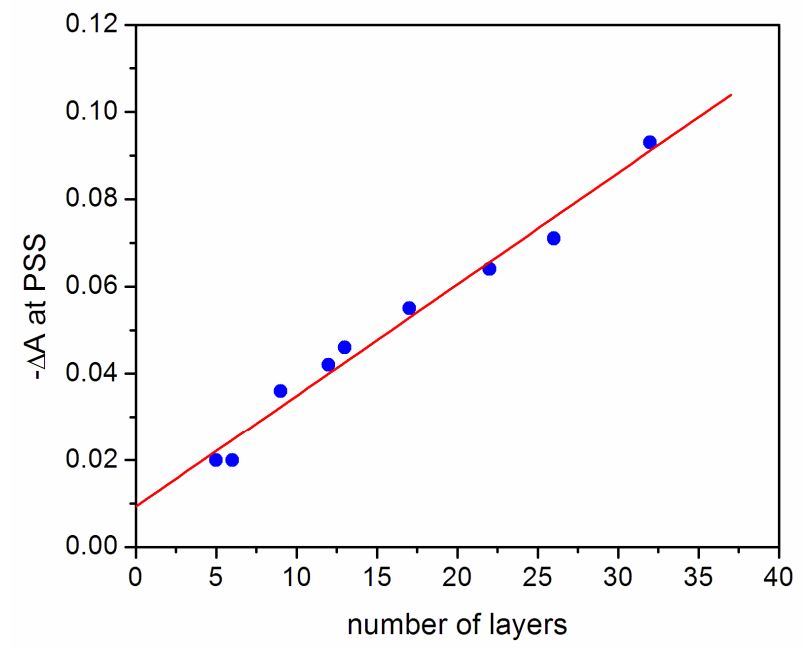

Figure 2.10. A plot of the difference in the absorbance at PSS and initial state as a function of the number of layers. The linear fit is $y=0.0093+0.0026 x$. 


\subsubsection{Effect of Azobenzene Packing Density}

In self-assembled monolayers, in particular on flat surfaces, steric constraint between neighboring molecules can impede the photoisomerization process. Evans et al. showed that single-component self-assembled monolayer containing only azobenzenederived alkanethiols on gold substrate do not photoisomerize ${ }^{[18]}$ Photoswitching behavior, however, can be restored when spacer molecules are incorporated to give a mixed monolayer. Figure 2.11 shows examples of the alkanethiol, corresponding spacer molecule and an optimized mixed-monolayer system. ${ }^{[19]}$ For colloids, the degree of steric hindrance depends on the nature of the substrate surface which in turn governs the packing order of the monolayer. The enhancements shown in Fig. 2.9 are in general lower than theoretical prediction, giving speculations on the role of steric hindrance in the azo- $\mathrm{SiO}_{2}$-o system. Therefore spacer molecules were incorporated in azo- $\mathrm{SiO}_{2}-\mathrm{O}$ as shown in Scheme 2.2, and their effect studied on the photoisomerization.

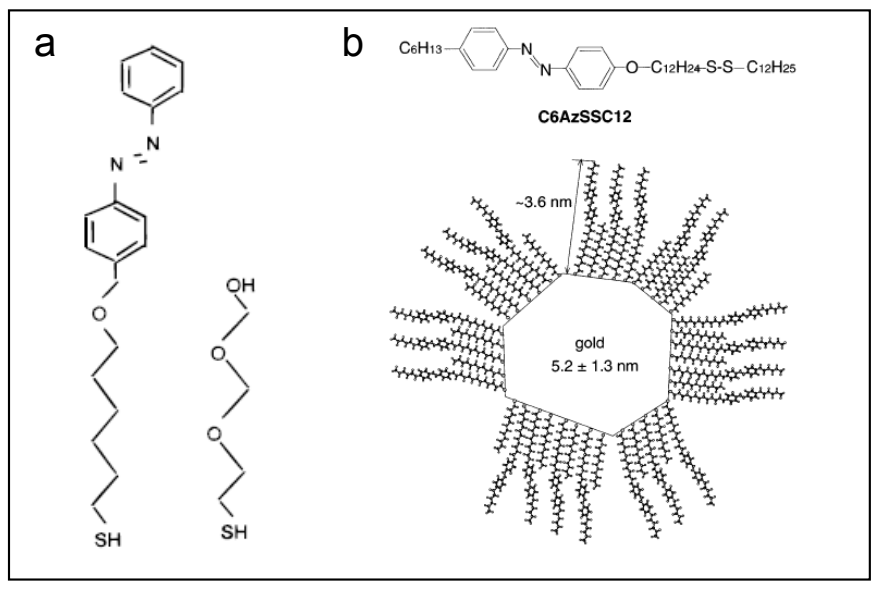

Figure 2.11. Photoswitchable gold substrate can be obtained from a mixed-monolayer containing azobenzene alkanethiol and spacer molecule (a). An optimized photoswitchable gold nanoparticle system using unsymmetrical azobenzene disulfides (b). (image reprinted with permission from ref. [18] and [19]). Copyright American Chemical Society. 
Figure 2.12 shows that by reacting $\mathrm{SiO}_{2}$-o with solutions containing mixtures of the spacer and azobenzene, the absorbance of the film due to $\pi-\pi^{*}$ transition of azobenzene decreases as the fraction of spacer molecules increases. The spacer effectively dilutes the density of azobenzene on $\mathrm{SiO}_{2}-\mathrm{O}$. The kinetics of trans-cis photoisomerization of these films, however were all comparable to that of pure azo- $\mathrm{SiO}_{2}$ o. The spacer does not increase the rate of the reaction suggesting that the azobenzene molecules are rather disordered on the $\mathrm{SiO}_{2}-\mathrm{O}$ and steric hindrance is negligible in transcis isomerization. This observation is understandable given the amorphous nature and surface roughness of the $\mathrm{SiO}_{2}$ spheres, and the random distribution of the hydroxyl groups that are used for anchoring the molecules. On the other hand, an improvement on the initial reversibility of the azobenzenes was found upon incorporation of more than $25 \%$ of spacer molecules (fraction of spacer can be estimated from the absorbance of the

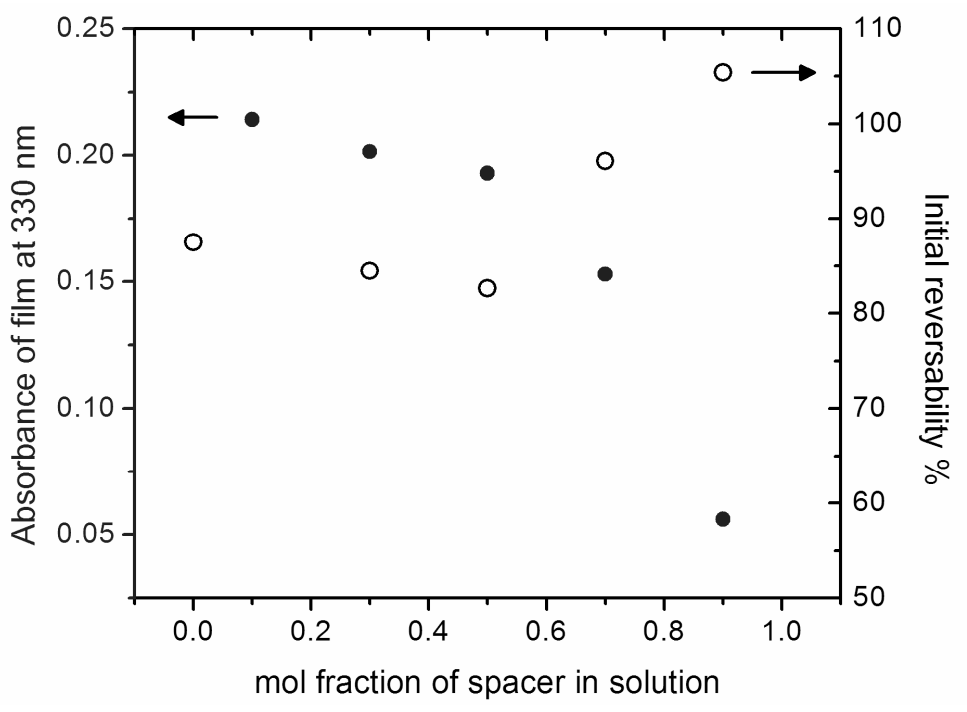

Figure 2.12. Absorbance of the films and reversibility of azobenzene on $\mathrm{SiO}_{2}$ opal of $85 \mathrm{~nm}$ spheres after dilution with the spacer. 
film). For azo-SiO $\mathrm{S}_{2}$-o without any spacer, the samples typically show a reversibility of $80-90 \%$ after the first cycle. By diluting the surface density of azobenzene with the spacer, a complete reversibility can be achieved. It can be concluded that incorporating the spacer affects the back reaction while having limited effect on the forward reaction. Although the details of the mechanisms are still under debate, it may be postulated that the reverse isomerization requires more space and therefore by reducing steric hindrance via the addition of spacer molecule, the cis-trans reaction proceeds to completion.

\subsection{Conclusions}

This work has demonstrated the effects of photonic properties on the photochemistry of organic molecules chemically tethered to the surface of a photonic crystal. The enhancements in a photochemical reaction from slow photons at both the red- and blue-edge of the photonic stop band were traced out for the first time. The present study provides insight to the rapidly developing field of optically enhanced chemical processes and demonstrates the versatility of slow photons at enhancing photochemistry of a wide range of systems.

\subsection{References}

[1] G. S. Hartley, J. Chem. Soc. 1938, 633.

[2] H. Rau, in Photoreactive organic thin films, (Eds: Z. Sekkat, W. Knoll), Elsevier Science, 2002, 3 .

[3] M. L. Tiago, S. Ismail-Beigi, S. G. Louie, J. Chem. Phys. 2005, 122. 
[4] D. S. Sidhaye, S. Kashyap, M. Sastry, S. Hotha, B. L. V. Prasad, Langmuir 2005, $21,7979$.

[5] M. Ueda, N. Fukushima, K. Kudo, K. Ichimurak, J. Mater. Chem. 1997, 7, 641.

[6] J. Eastoe, M. S. Dominguez, H. Cumber, P. Wyatt, R. K. Heenan, Langmuir 2004, $20,1120$.

[7] J. Eastoe, A. Vesperinas, Soft Matter 2005, 1, 338.

[8] Y. L. Yu, M. Nakano, T. Ikeda, Nature 2003, 425, 145.

[9] M. Yamada, M. Kondo, J.-i. Mamiya, Y. Yu, M. Kinoshita, C. J. Barrett, T. Ikeda, Angew. Chem. Int. Ed. 2008, 47, 4986.

[10] F. Fleischhaker, A. C. Arsenault, V. Kitaev, F. C. Peiris, G. Von Freymann, I. Manners, R. Zentel, G. A. Ozin, J. Am. Chem. Soc. 2005, 127, 9318.

[11] S. Shinkai, T. Minami, Y. Kusano, O. Manabe, J. Am. Chem. Soc. 1983, 105, 1851.

[12] S. Shinkai, K. Miyazaki, O. Manabe, J. Chem. Soc., Perkin Trans. 1 1987, 449.

[13] H. Asanuma, T. Ito, T. Yoshida, X. G. Liang, M. Komiyama, Angew. Chem. Int. Ed. 1999, 38, 2393.

[14] L. Chi, O. Sadovski, G. A. Woolley, Bioconj. Chem. 2006, 17, 670.

[15] G. A. Woolley, A. S. I. Jaikaran, M. Berezovski, J. P. Calarco, S. N. Krylov, O. S. Smart, J. R. Kumita, Biochem. 2006, 45, 6075.

[16] J. J. De Lange, J. M. Robertson, I. Woodward, Proc. Roy. Soc. London, Sect. A 1939, 171, 398.

[17] I. Conti, M. Garavelli, G. Orlandi, J. Am. Chem. Soc. 2008, 130, 5216. 
[18] S. D. Evans, S. R. Johnson, H. Ringsdorf, L. M. Williams, H. Wolf, Langmuir 1998, 14, 6436.

[19] A. Manna, P. L. Chen, H. Akiyama, T. X. Wei, K. Tamada, W. Knoll, Chem. Mater. 2003, 15, 20.

[20] H. Ge, G. Wang, Y. He, X. Wang, Y. Song, L. Jiang, D. Zhu, Chem. Phys. Chem. 2006, 7, 575.

[21] M. Kamenjicki, I. K. Lednev, A. Mikhonin, R. Kesavamoorthy, S. A. Asher, Adv. Func. Mater. 2003, 13, 774.

[22] P. Jiang, J. F. Bertone, K. S. Hwang, V. L. Colvin, Chem. Mater. 1999, 11, 2132.

[23] J. F. Galisteo-Lopez, M. Galli, M. Patrini, A. Balestreri, L. C. Andreani, C. Lopez, Phys. Rev. B 2006, 73, 125103.

[24] G. von Freymann, S. John, S. Wong, V. Kitaev, G. A. Ozin, Appl. Phys. Lett. 2005, 86, 053108.

[25] J. F. Galisteo-Lopez, E. Palacios-Lidon, E. Castillo-Martinez, C. Lopez, Phys. Rev. B 2003, 68, 115109.

[26] M. A. Kaliteevski, J. Manzanares Martinez, D. Cassagne, J. P. Albert, Phys. Rev. B 2002, 66, 1131011. 


\title{
CHAPTER 3
}

\section{Slow-Photon Enhanced Photodegradation Efficiency of Titania}

(Reproduced in part with permission from Adv. Mater. 2006, 18, 1915. Copyright 2006 Wiley-VCH Verlag GmbH \& Co. KGaA.)

\begin{abstract}
It has been more than a decade since the inception of photonic crystal and the establishment of the theory for slow photon and photonic band-edge enhancement. Since then, various researchers investigated the photonic properties for enhancing the absorption or emission of dyes ${ }^{[1]}$ and quantum dots. ${ }^{[2]}$ However, the feasibility of using slow photons to increase the yield of a photochemical process, in which the enhanced absorption actually leads to a chemical reaction or "work", remained relatively unexplored. Therefore, this work aims to demonstrate an optically amplified photochemical process using anatase Titania $\left(\mathrm{TiO}_{2}\right)$ as the photoactive material. In this chapter, photoactive anatase $\mathrm{TiO}_{2}$ is molded into a three-dimensional colloidal photonic crystal and its photodegradation efficiency is investigated with respect to the photonic properties. Slow photon optical amplification in the nanocrystalline inverse $\mathrm{TiO}_{2}$ opal (denoted $\mathrm{i}-\mathrm{nc}-\mathrm{TiO}_{2}-\mathrm{o}$ ) is demonstrated via carefully designed solid-state photodegradation experiments where the photo-oxidation of adsorbed dye under monochromatic and whitelight irradiation is monitored. When the slow-photon wavelength is optimized with respect to the electronic excitation energy of $\mathrm{i}-\mathrm{nc}-\mathrm{TiO}_{2}-\mathrm{o}$, the photo-oxidation rate of the dye is doubled compared to conventional nanocrystalline $\mathrm{TiO}_{2}\left(\mathrm{nc}-\mathrm{TiO}_{2}\right)$ due to increased
\end{abstract}


absorption that lead to a higher population of electron-hole pairs. As anatase $\mathrm{TiO}_{2}$ is widely used for degrading organic pollutants, the finding has immediate technological importance.

\subsection{Introduction}

Titania exists in three polymorphs: brookite, rutile, and anatase. The three crystal structures differ in the arrangement and connectivity of the octahedral building blocks (TiO6), with anatase having chains of four-edge sharing octahedra and rutile having chains of two-edge sharing octahedra. Figure 3.1 shows the crystal structure of the three polymorphs. The thermodynamically most stable state of $\mathrm{TiO}_{2}$ is rutile, which is less photoactive than anatase. Rutile $\mathrm{TiO}_{2}$ is commonly used as an opacifier in various consumer products such as paints and cosmetics. Anatase $\mathrm{TiO}_{2}$, on the other hand, is 2.61 $\mathrm{kJ} / \mathrm{mol}$ higher in enthalpy than rutile but has the lowest surface energy of all three

polymorphs. ${ }^{[3]}$ When the grain size of $\mathrm{TiO}_{2}$ is decreased to tens of nanometers and the surface area is increased, an interesting energy crossover takes place where anatase becomes the most stable form of $\mathrm{TiO}_{2}$. Figure 3.2 shows the total free energy of the three polymorphs with respect to the surface area of $\mathrm{TiO}_{2}$. As the particle size decreases to $<15$ $\mathrm{nm}$, the surface free energy becomes a significant part of the total free energy and the minimization of which therefore leads to the stability of anatase over rutile. To the advantage of many applications, high surface area combined with high photoactivity is desirable thus making nanocrystalline anatase $\mathrm{TiO}_{2}$ an extremely valuable material. 


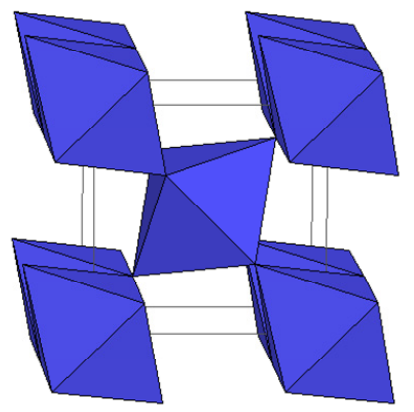

Rutile

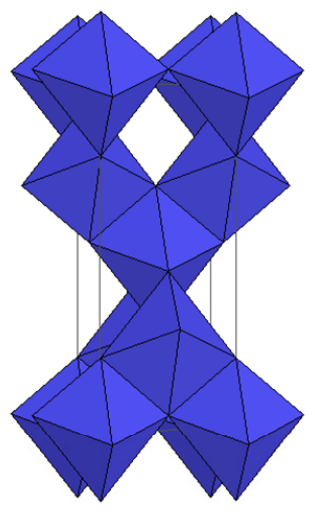

Anatase

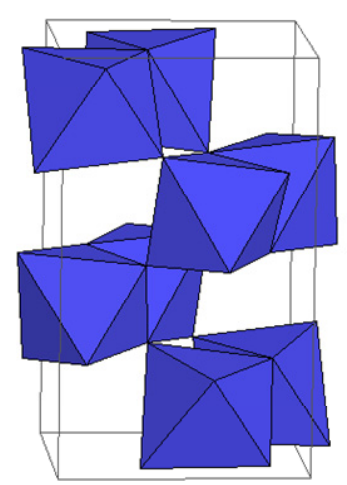

Brookite

Figure 3.1. Crystal structures of the polymorphs of $\mathrm{TiO}_{2}$.

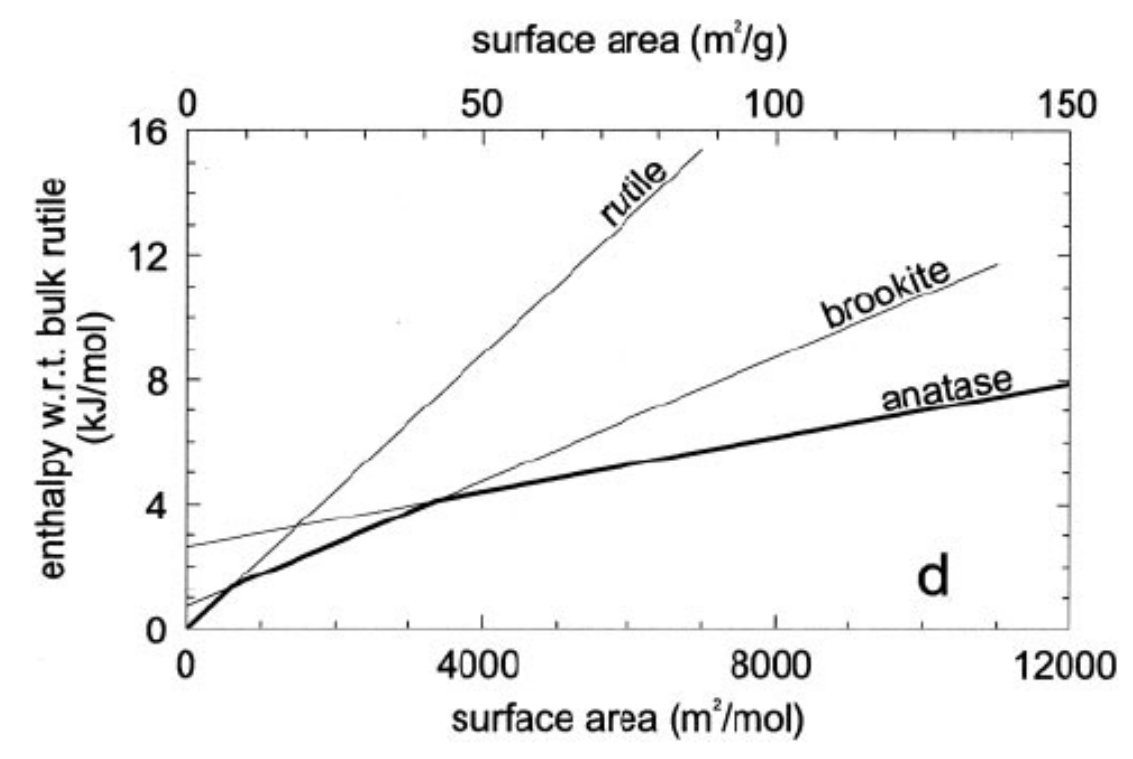

Figure 3.2. Graphical representation of the enthalpy of nanocrystalline $\mathrm{TiO}_{2}$ with respect to bulk rutile as a function of surface area. The darker line segments indicate the energetically stable phase. The phase stability crossover is observed from rutile to brookite to anatase with increasing surface area. (image reprinted with permission from ref. [3]). Copyright National Academy of Sciences, U.S.A. 


\subsubsection{Optical and Electronic Properties of Anatase $\mathrm{TiO}_{2}$}

Anatase $\mathrm{TiO}_{2}$ is a semiconductor with an indirect-electronic bandgap of $3.2 \mathrm{eV}$. The energy level diagram in Fig. 3.3a shows several allowed direct transitions and allowed but phonon-assisted indirect transitions between the valence and conduction band states in $\mathrm{TiO}_{2} \cdot{ }^{[4]}$ The energy of the valence and conduction band is at $-7.8 \mathrm{eV}$ and $4.4 \mathrm{eV}$ respectively (Fig. 3.3b), with the valence band comprising of mostly $\mathrm{O}^{2-} 2 \mathrm{p}$ states and the conduction band of mainly $\mathrm{Ti}^{4+} 3 \mathrm{~d}$ states. However, $\mathrm{TiO}_{2}$ does not have full ionicity and significant amount of hybridization between $\mathrm{O}^{2-}$ and $\mathrm{Ti}^{4+}$ states occur leading to a covalent-bond character and wide electronic bands. The energy levels of the valence and conduction bands make the photogenerated electrons highly reducing and holes highly oxidizing. For this reason, anatase $\mathrm{TiO}_{2}$ is able to degrade a variety of organic

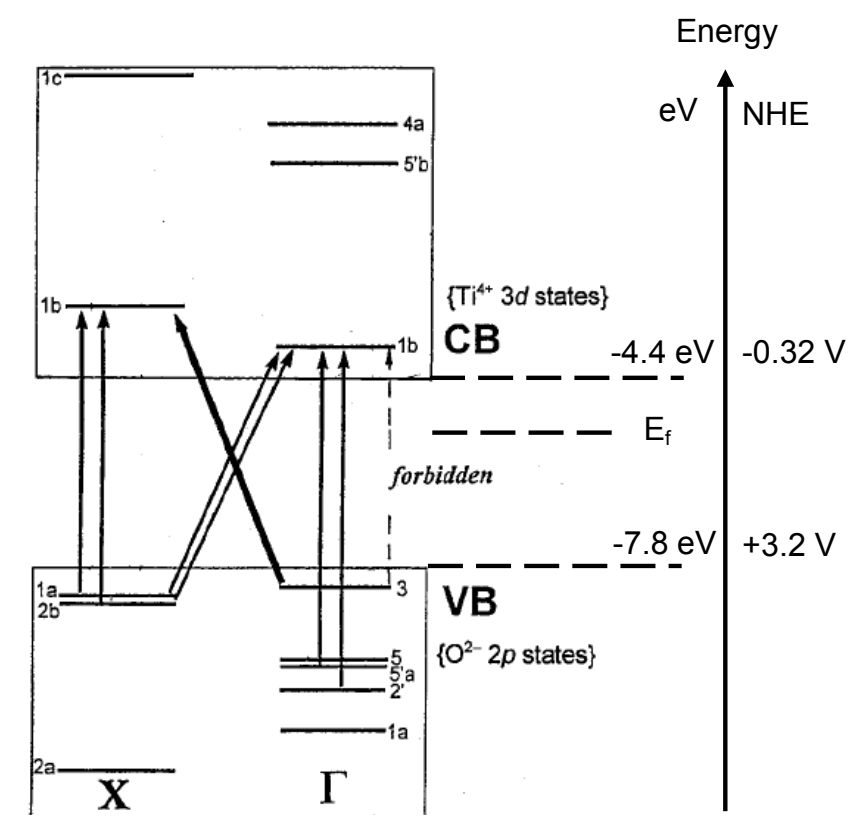

Figure 3.3. Energy level band diagram of anatase $\mathrm{TiO}_{2}$ showing the electronic transitions between the valence and conduction band. The energy scale in $\mathrm{eV}$ and normal hydrogen electrode is shown on the right. (image reprinted with permission from ref. [4]). Copyright American Physical Society. 
compounds making it ideal for environmental applications. ${ }^{[5,6]}$ However, the large bandgap also brings a shortcoming as only a few percent of solar radiation has energy greater than $3.2 \mathrm{eV}$ and can be absorbed by anatase $\mathrm{TiO}_{2}$. Hence increasing the absorption of anatase is highly important and relevant.

\subsubsection{Photocatalysis with Anatase $\mathrm{TiO}_{2}$}

Upon absorbing UV light (energy $>3.2 \mathrm{eV}$ ), electron-hole pairs are produced in $\mathrm{TiO}_{2}$ within a few femtoseconds. With no inactive background absorption, the quantum yield for the generation of electron-hole pair is 1 . However, the quantum yield of photocatalysis, defined as

$$
\Phi_{\lambda}=\frac{\text { moles of reactant transformed }}{\text { moles of photons (Einsteins) absorbed by catalyst at } \lambda}
$$

is usually on the order of 0.1 for $\mathrm{TiO}_{2}{ }^{[7,8]}$, meaning that most of the photogenerated electron-hole pairs do not participate in the reaction. Instead, they undergo several different recombination pathways in the bulk or on the surface. ${ }^{[9]}$ For example, the electron can be trapped by lattice defects as $\mathrm{Ti}^{\mathrm{III}}$ or by adsorbed $\mathrm{O}_{2}$ to form superoxide radical anion $\mathrm{O}_{2}^{-\bullet}$. The hole can be trapped by lattice defects as $\mathrm{Ti}^{\mathrm{IV}}-\mathrm{O}^{-\bullet}-\mathrm{Ti}^{\mathrm{IV}}$ or by surface $\mathrm{OH}^{-}$or $\mathrm{H}_{2} \mathrm{O}$ to give the surface-bound hydroxide radical $\left(\mathrm{Ti}-\mathrm{OH}^{\bullet}\right)$. Both the hydroxide and superoxide radicals are highly reactive and are the main species responsible for the degradation of organic compounds by $\mathrm{TiO}_{2} .^{[10,11]}$ The average electron-hole pair lifetime is $\sim 30 \mathrm{~ns}$ with the hole having higher mobility and diffusion length comparable to the size of the nanocrystals. ${ }^{[12]}$ Direct photooxidation of organic molecules by the hole is possible on the $100 \mathrm{~ns}$ scale, while direct photoreduction by the electron is a very slow process on the order of milliseconds. As the main recombination 
pathways occur on the surface, the surface chemistry of $\mathrm{TiO}_{2}$ is crucial in governing the charge carrier dynamics. In fact $\mathrm{TiO}_{2}$ made by different synthetic methods show varying lifetimes of the electron-hole, with the commercially available Degussa P25 exhibiting one of the highest charge-separation lifetimes ${ }^{[13]}$ and therefore regarded as the most efficient photocatalyst.

\subsubsection{Surface Chemistry of $\mathrm{TiO}_{2}$}

Metal and oxide ions on the surface of a particle have lower coordination than those in the bulk. To increase the coordination number and decrease the chemical potential of the surface ions, water adsorbs and dissociates on the surface to give hydroxyl groups. Chemisorption of $\mathrm{H}_{2} \mathrm{O}$ on $\mathrm{TiO}_{2}$ occurs on 5 -coordinated $\mathrm{Ti}^{4+}$ that are Lewis acids and 2-coordinated $\mathrm{O}^{2-}$ that are Lewis bases as shown in Fig. 3.4. ${ }^{[14]}$

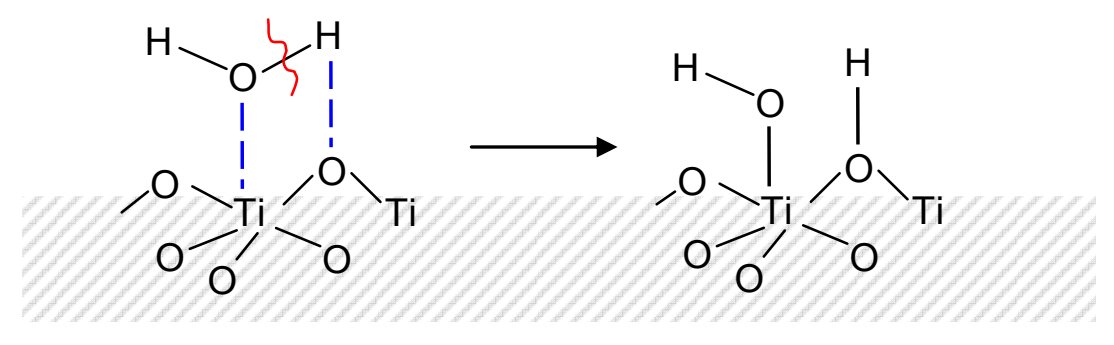

Figure 3.4. Illustration of the proposed mechanism of chemisorption of $\mathrm{H}_{2} \mathrm{O}$ on the surface of $\mathrm{TiO}_{2}$ to yield charge-active hydroxyl groups.

The resultant hydroxyl groups can ionize in solution to give charges on the surface according to the following:

$$
\mathrm{M}-\mathrm{O}^{-}+\mathrm{H}_{3} \mathrm{O}^{+} \rightleftharpoons \mathrm{M}-\mathrm{OH}+\mathrm{H}_{2} \mathrm{O} \rightleftharpoons \mathrm{M}_{2} \mathrm{OH}_{2}^{+}+\mathrm{OH}^{-}
$$


The hydroxyl groups can be mono, di, or tricoordinated depending on the structure and crystal face of the metal oxide, and on average there are $5-7$ charge-active sites per $\mathrm{nm}^{2}$ of $\mathrm{TiO}_{2} \cdot{ }^{[15]}$ The surface charge of $\mathrm{TiO}_{2}$, therefore, can be controlled by changing the $\mathrm{pH}$ of the solution it is in contact with. The point of zero charge of $\mathrm{TiO}_{2}$ has been reported between $\mathrm{pH}$ of 4 and 7 , above which the surface is negatively charged and below which it is positively charged. The ability to control the surface charge of $\mathrm{TiO}_{2}$ facilitates the adsorption of molecules via electrostatic interaction - a key factor in the photocatalytic efficiency.

\subsubsection{Enhancing the Absorption of Anatase $\mathrm{TiO}_{2}$}

As only a few percent of solar irradiation has energy greater than $3.2 \mathrm{eV}$ that can be absorbed by anatase $\mathrm{TiO}_{2}$ to generate the electrons and holes, an extensive amount of work has been devoted to lowering the bandgap through chemical doping or sensitization. The most promising results have come in the last decade where elemental doping with $\mathrm{N}$, $\mathrm{C}$ and $\mathrm{S}$ have yielded $\mathrm{TiO}_{2}$-based photocatalysts that absorb in the visible range (up to $500 \mathrm{~nm}) \cdot{ }^{[16-18]}$ Aside from chemical doping, increasing the active surface area by making $\mathrm{TiO}_{2}$ mesoporous ${ }^{[19-21]}$ and enhancing electron-hole separation via the incorporation of metal nanoparticles have also been actively pursued. ${ }^{[22,23]}$

On the other hand, with the inception of various photonic structures and collaboration between physicists and material chemists, there is an emerging field of optical amplification of absorption by increasing the path-length of light through the photoactive material. Some examples on incorporating designed photonic structures with photoactive materials are outlined in Section 1.6. Alternatively, Meseguer and co- 
workers increased multiple light scattering in $\mathrm{TiO}_{2}$ film via a photonic sponge structure ${ }^{[2]}$; this effect can also be achieved by incorporating large $\mathrm{TiO}_{2}$ particles or spherical voids (Fig. 3.5). ${ }^{[25]}$
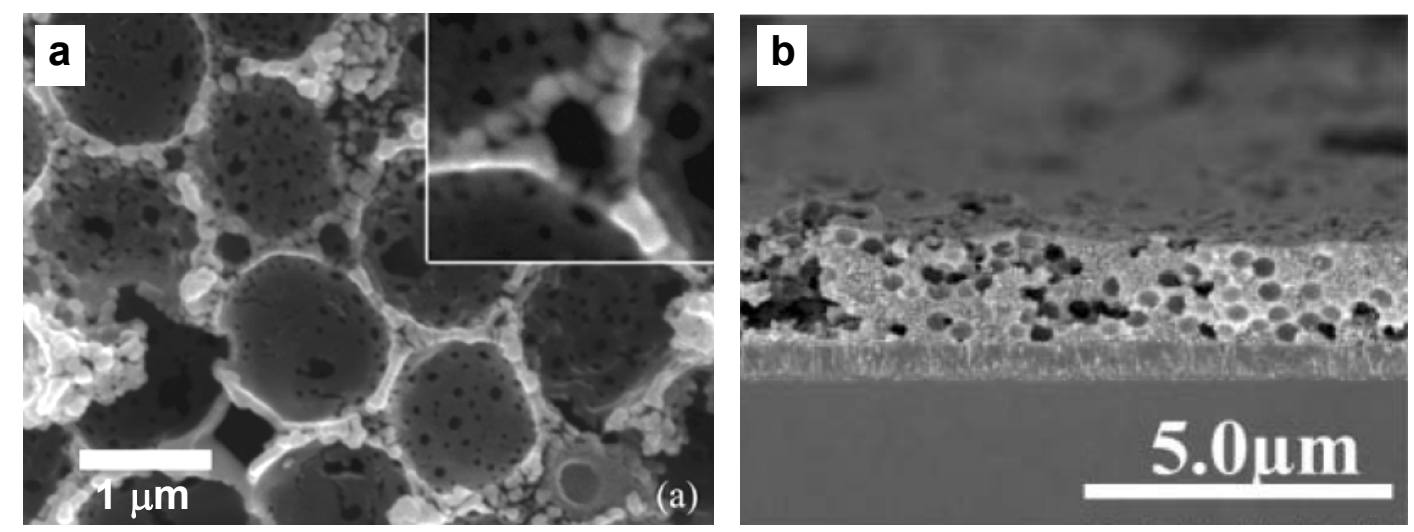

Figure 3.5. Different approaches for increasing the path length of light through $\mathrm{TiO}_{2}$ via random light scattering in photonic sponge structure (a) and incorporation of spherical voids (b). (images reprinted with permission from ref. [24] and [25]). Copyright Royal Society of Chemistry.

In this chapter, the photoactivity of $\mathrm{i}-\mathrm{nc}-\mathrm{TiO}_{2}-\mathrm{O}$ with photonic properties at different energies is investigated with respect to that of a non-structured nc- $\mathrm{TiO}_{2}$. By optimizing the overlap of the photonic energy with the anatase absorption band-edge, the probability of absorbing photons in $\mathrm{i}-\mathrm{nc}-\mathrm{TiO}_{2}-\mathrm{o}$ relative to $\mathrm{nc}-\mathrm{TiO}_{2}$ is increased, leading to a larger population of electron-hole pairs and a more efficient photo-oxidation.

\subsection{Experimental Methods}

\subsubsection{Fabrication of Inverse $\mathrm{TiO}_{2}$ Opals}

The films of i-nc- $\mathrm{TiO}_{2}$-o with stop-band maxima centered at 280, 300, 325, 345, 370,430 and $500 \mathrm{~nm}$ were prepared by infiltrating polystyrene sphere opal templates of 
diameters 130, 150, 180, 210, 240, 300 and $380 \mathrm{~nm}$ with titanium butoxide $\left(\mathrm{Ti}(\mathrm{OBut})_{4}\right)$. The templates for the inverse opals had thicknesses varying from 15 to 20 layers of polystyrene spheres self-assembled via the solvent evaporation method. Infiltration was done by placing the opal templates in ethanol solutions containing $0.5-1 \mathrm{vol} \%$ of $\mathrm{Ti}(\mathrm{OBut})_{4}$ under low pressure. As ethanol evaporated, the alkoxide precursor filled the voids and hydrolyzed there. The infiltrated samples were then exposed to air plasma in order to remove the polystyrene spheres while maintaining good structural integrity. Finally, the amorphous samples were calcined at $450{ }^{\circ} \mathrm{C}$ for 4 hours to produce pure anatase phase.

\subsubsection{Fabrication of Reference Nanocrystalline $\mathrm{TiO}_{2}$}

Reference nc- $\mathrm{TiO}_{2}$ films were obtained from two methods: i) depositing $\mathrm{Ti}(\mathrm{OBut})_{4}$ on glass slide via the evaporation of ethanol solvent under reduced pressure, as in the infiltration method followed by the same air plasma and calcination treatment; ii) redepositing fine powder of crushed-i-nc- $\mathrm{TiO}_{2}-\mathrm{o}$ into a film. Briefly, the crushed i-nc$\mathrm{TiO}_{2}$-o was prepared by sonicating 8 inverse opals (of different air sphere sizes) in ethanol to break up the inverse opal structure. A glass substrate was then placed in the suspension and the powder redeposited as a film while ethanol evaporated.

\subsubsection{Materials Characterization}

\subsubsection{Scanning (Transmission) Electron Microscopy}

Scanning electron microscopy (SEM) images were obtained using Hitachi S-5200 operating at $1 \mathrm{kV}$ for films on glass substrate, or at $30 \mathrm{kV}$ for films scraped-off onto a 
carbon-coated copper grid. High-resolution scanning transmission electron microscopy (STEM) images were obtained using Hitachi HD-2000 in the Z-contrast mode at an accelerating voltage of $200 \mathrm{kV}$ and emission current of $50 \mu \mathrm{A}$.

\subsubsection{X-ray Diffraction and Raman Spectroscopy}

The crystal phase and particle size of the films were analyzed by X-ray diffraction (XRD) using a Siemens D5000 diffractometer and $\mathrm{Cu}-\mathrm{k}_{\alpha}$ line as the X-ray source. The Rietveld refinement was carried out with Bruker AXS general profile fitting software Topas $^{\mathrm{TM}}$. Raman spectra were obtained at ambient temperature on a Raman spectrometer with a frequency-doubled Nd:YAG laser source $(532 \mathrm{~nm})$.

\subsubsection{UV-Vis Spectroscopy}

Optical spectra were measured using a Perkin Elmer UV/VIS/NIR spectrometer Lambda 900. Normal transmission mode was used to measure the extinction of the films while the diffuse reflectance and real absorbance spectra were obtained using an integrating sphere detector. For the latter, a highly reflecting plate of $\mathrm{BaSO}_{4}$ was placed at the back of the film so that the probe light passes through the sample twice and only light absorbed by the sample will be absent in the detection process. ${ }^{[11]}$

\subsubsection{Dye Adsorption Study}

The films were immersed in methylene blue solutions with increasing concentrations of $0.5-7 \mu \mathrm{M}$ at $\mathrm{pH}$ of 8 (adjusted with ammonia) for $60 \mathrm{~min}$. The excess dye was rinsed away with water and ethanol. After drying, the extinction of dye on the films was measured. The peaks in the extinction spectra were deconvoluted using Microcal Origin software to give the extinction of the monomer molecules at $660 \mathrm{~nm}$. 


\subsubsection{Photodegradation Experiment}

After dye adsorption, the films were irradiated with light and the dye extinction monitored over time. Monochromatic irradiation was carried out using a 180W Xe lamp in combination with a monochromator. White light irradiation was performed using a 120W Xe lamp with a neutral density filter that cuts off $<300 \mathrm{~nm}$. Extinction spectra were taken at 30 s intervals over a total time of three minutes for the white light photodegradation experiments and at 1-min intervals for 7 minutes for the monochromatic experiments. The decay rate constants were obtained from at least two different samples and averaged over a total of three runs or more to obtain statistically meaningful data. For angle-dependent photo-degradation experiments, the films were first irradiated at the specified angle off-normal of the films, and then spectral recordings normal to the films were obtained using the UV-Vis spectrometer. Additional control experiments showed negligible degradation of dye on meso- $\mathrm{SiO}_{2}$ under white light and on $\mathrm{i}-\mathrm{nc}-\mathrm{TiO}_{2}-\mathrm{o}$ under visible light $(>400 \mathrm{~nm})$, thus excluding the possibility of dye bleaching and back sensitization as the pathway for degradation. Heating the films with the adsorbed dye at $70{ }^{\circ} \mathrm{C}$ also resulted in little thermal degradation in the time frame of our experiments.

\subsection{Characterization of Inverse $\mathrm{TiO}_{2}$ Opals}

\subsubsection{Optical Properties}

Polystyrene opals with sphere sizes ranging from 130 to $380 \mathrm{~nm}$ were used as templates for making i-nc- $\mathrm{TiO}_{2}-\mathrm{o}$. Figure 3.6 shows the extinction spectra of the opal templates. Fabry-Perot fringes arising from the constructive interference of light at the top and bottom of the films are observed, indicative of the high quality of the templates. 
Upon infiltration with $\operatorname{Ti}(\mathrm{OBut})_{4}$, the Bragg reflection of the opal disappears due to the dielectric matching of the polystyrene spheres with porous amorphous $\mathrm{TiO}_{2}$. Using SWA to model the experimental optical spectra, the interstitial of the opal template is filled with $50 \mathrm{vol} \%$ of air and $50 \mathrm{vol} \%$ of amorphous $\mathrm{TiO}_{2}$ (refractive index of 2.1 from ellipsometry) upon infiltration. The infiltrated $\mathrm{TiO}_{2}$-PS opal composite was then treated in air plasma to remove the polystyrene spheres and calcined at $450{ }^{\circ} \mathrm{C}$ to yield nanocrystalline anatase $\mathrm{TiO}_{2}$. Figure 3.7 shows a comparison of the extinction spectra of the bare, infiltrated, plasma-treated and calcined inverse opal. After calcination, the framework of the inverse opal was determined to be $\sim 30$ vol $\%$ anatase $\mathrm{TiO}_{2}(\mathrm{RI}=2.7)$ and $\sim 70 \mathrm{vol} \%$ air, giving an overall $\sim 7 \mathrm{vol} \%$ of $\mathrm{TiO}_{2}$ in the film. The low filling fraction and shrinkage of $\mathrm{TiO}_{2}$ precursor was advantageous, such that $\mathrm{i}-\mathrm{nc}-\mathrm{TiO}_{2}-\mathrm{o}$ with stop-band energies well into the absorption of $\mathrm{TiO}_{2}$ may be obtained with template spheres no smaller than $130 \mathrm{~nm}$, which were synthesized surfactant-free with very good monodispersity. As a result, $\mathrm{i}-\mathrm{nc}-\mathrm{TiO}_{2}-\mathrm{o}$ with high optical quality that extends well into UV wavelengths is achieved for the first time.

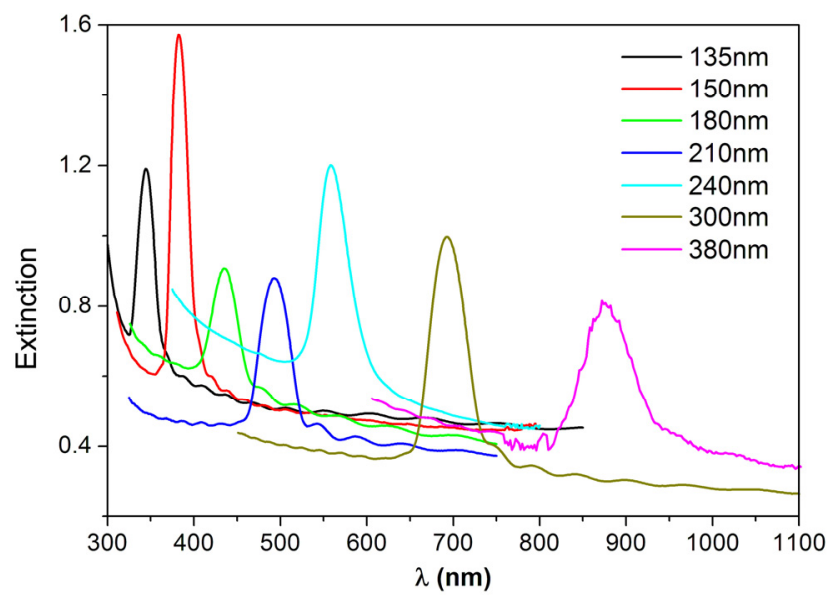

Figure 3.6. Extinction spectra of opal templates made from different polystyrene spheres (size indicated in the legend). 


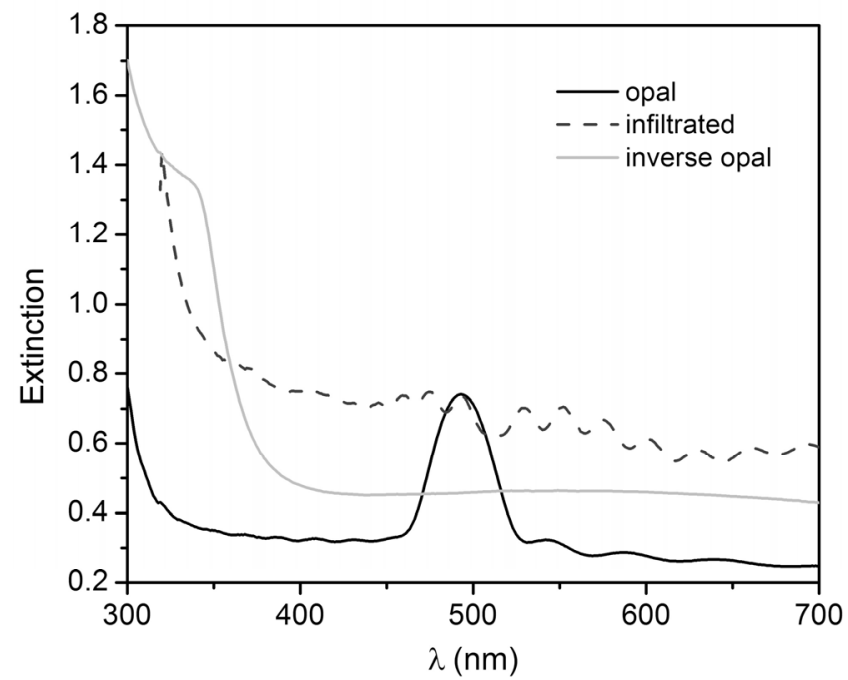

Figure 3.7. Extinction spectra of bare, infiltrated and inverse opal made from $210 \mathrm{~nm}$ spheres. The shoulder at $350 \mathrm{~nm}$ indicates the stop band of the $\mathrm{i}-\mathrm{nc}-\mathrm{TiO}_{2}-\mathrm{O}$.

To better characterize the photonic stop band, UV-Vis reflectance spectroscopy was employed. Figure 3.8 shows the reflectance spectra of $\mathrm{i}-\mathrm{nc}-\mathrm{TiO}_{2}-\mathrm{o}$ with different photonic stop-band energies measured in air and water. The inverse opals have stopbands centered at 280, 300, 325, 345, 370, 430 and $500 \mathrm{~nm}$ (herein the prefix before i-nc$\mathrm{TiO}_{2}-\mathrm{o}$ denotes the stop-band maximum of the inverse opal). Since the energy of the photonic stop-band for most $\mathrm{i}-\mathrm{nc}-\mathrm{TiO}_{2}$-o overlaps with that of the electronic band gap of nc- $\mathrm{TiO}_{2}$, the extinction spectra feature only a shoulder rather than a well-defined maximum (e.g. Fig. 3.7, spectrum in grey). However, by detecting in reflectance mode, Bragg reflection down to $325 \mathrm{~nm}$ can be seen and that of $300-\mathrm{i}-\mathrm{nc}-\mathrm{TiO}_{2}$-o is observable by filling the inverse opal structure with water to shift the stop-band to $325 \mathrm{~nm}$. The energy of the slow photons of interest (at the red-edge of the stop band) is approximated by the arrows for each i-nc- $\mathrm{TiO}_{2}-\mathrm{o}$ shown in Fig. 3.8a. Moreover, a linear relationship between stop-band wavelength (in the range of $300-400 \mathrm{~nm}$ ) of the inverse opals and 
template sphere size was observed (Fig. 3.9); this linear relation was used to interpolate the stop-band position of the $280-\mathrm{i}-\mathrm{nc}-\mathrm{TiO}_{2}-\mathrm{O}$.
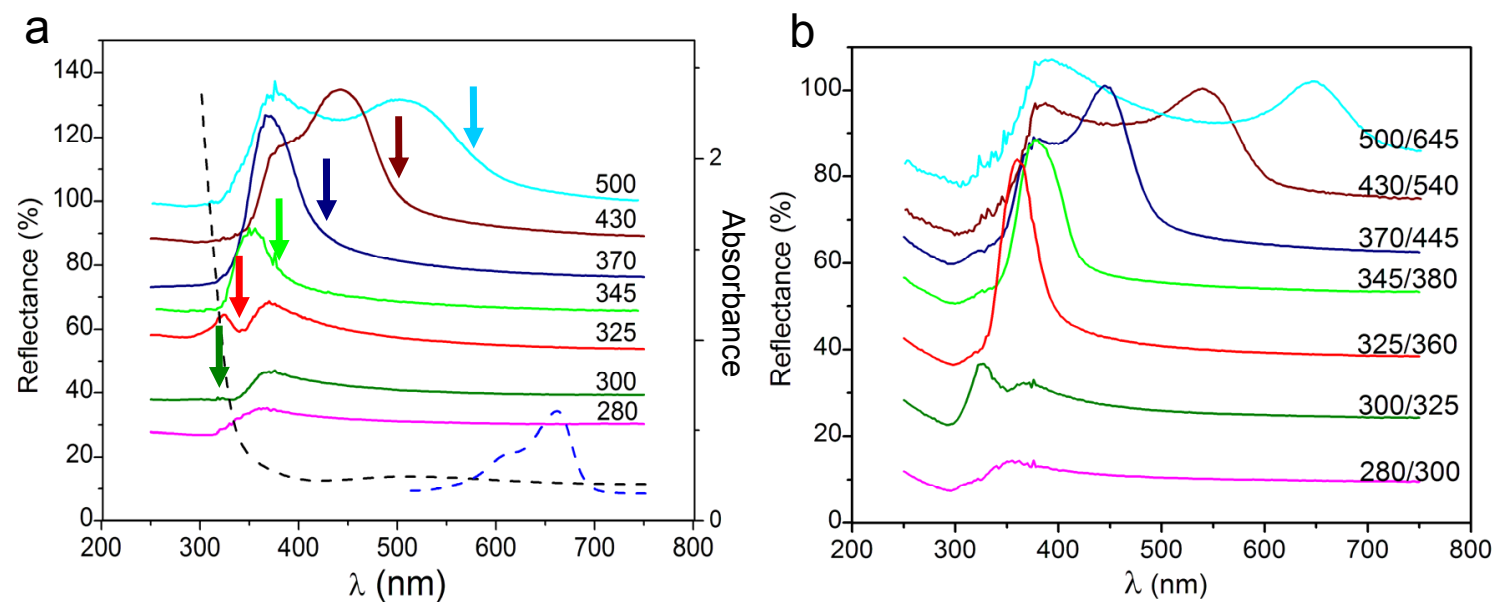

Figure 3.8. Reflectance spectra of $\mathrm{i}-\mathrm{nc}-\mathrm{TiO}_{2}-\mathrm{o}$ in air (a) and water (b). The positions of the stop band are labeled (first/second in (b) correspond to stop band position in air and water, respectively). The energy of the slow photons for each sample is approximated by the arrows. The absorption of nc- $\mathrm{TiO}_{2}$ and methylene blue are shown as dashed lines in (a).

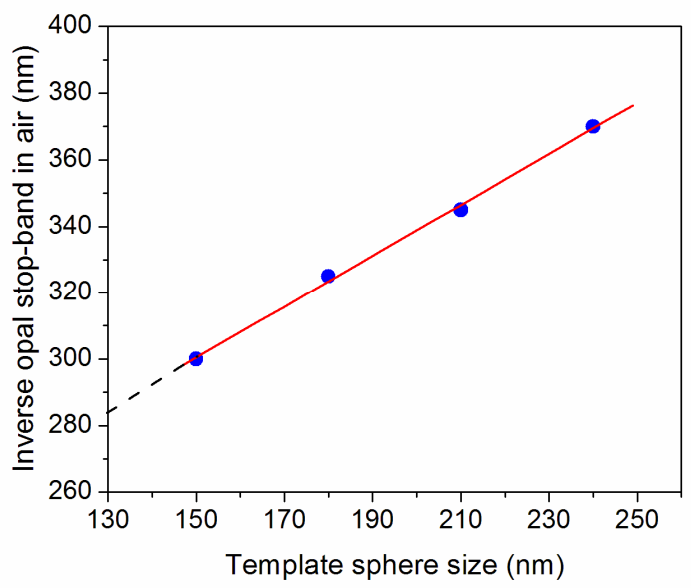

Figure 3.9. Linear relationship between stop band position and template sphere size. 
Due to the scattering nature of $\mathrm{i}-\mathrm{nc}-\mathrm{TiO}_{2}-\mathrm{o}$, the real absorbance cannot be determined from the transmission spectra, where by definition absorbance is $-\log \mathrm{T}$ for a non-scattering sample. As a result, extinction, which includes both absorbed and scattered light, instead of pure absorbance of $\mathrm{i}-\mathrm{nc}-\mathrm{TiO}_{2}-\mathrm{o}$ was measured by the conventional method. However, Sun and Bolton demonstrated that diffuse reflectance obtained from integrating sphere when taken with a highly reflecting reference at the rear of the sample corresponds to the real absorbance of a scattering sample. ${ }^{[11]}$ Figure 3.10 shows the absorbance of $\mathrm{i}-\mathrm{nc}-\mathrm{TiO}_{2}-\mathrm{o}$ obtained using this method. For 300- and 325-i-nc- $\mathrm{TiO}_{2}-\mathrm{o}$, the onset of the absorption edge is clearly red-shifted with respect to 280 - $\mathrm{i}-\mathrm{nc}-\mathrm{TiO}_{2}-\mathrm{o}$ (which does not have any photonic effect). The $280-\mathrm{i}-\mathrm{nc}-\mathrm{TiO}_{2}-\mathrm{o}$ is a suitable reference for comparison as the amount of $\mathrm{TiO}_{2}$ is comparable to the other $\mathrm{i}-\mathrm{nc}-\mathrm{TiO}_{2}$-o while lacking photonic properties due to the small diffraction periodicity. On the other hand, a slight decrease in absorbance at $370 \mathrm{~nm}$ due to the Bragg reflection can be observed for $370-\mathrm{i}-$ nc- $\mathrm{TiO}_{2}$-o. To better demonstrate the changes in the absorbance due to photonic effects, the differences in absorbance of 300-, 325-, 345- and 370-i-nc- $\mathrm{TiO}_{2}-\mathrm{o}$ with respect to 280-i-nc- $\mathrm{TiO}_{2}-\mathrm{O}$ are shown in Fig. 3.10b. Enhanced absorption at $320-370 \mathrm{~nm}$ is clearly observed in 300- and 325-i-nc- $\mathrm{TiO}_{2}$-o showing the red-edge slow photon enhancement for these two samples. Note that while theory predicts a very narrow spectral range that slow photons are found $(\Delta \lambda / \lambda \sim 1-2 \%)^{[26]}$, Fig. 3.10b shows the enhancement spans $\sim 50$ $\mathrm{nm}(\Delta \lambda / \lambda>10 \%)$. On the contrary, for $345-$ and $370-\mathrm{i}-\mathrm{nc}-\mathrm{TiO}_{2}-\mathrm{o}$ both a dip and a small increase in absorption are observed at wavelengths corresponding to their stop band reflection and slow photon enhancement. 
a

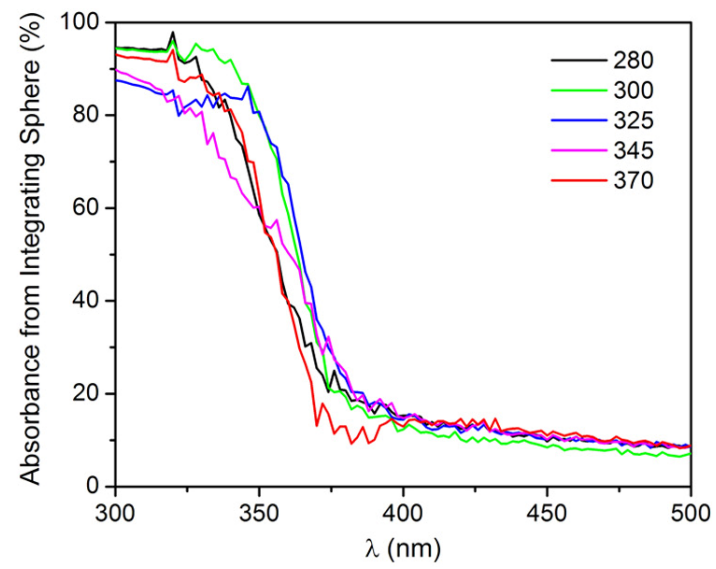

b

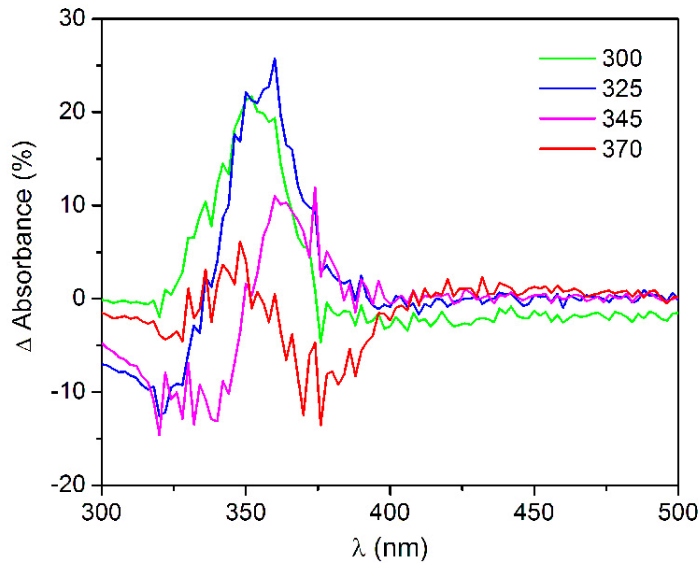

Figure 3.10. Absorbance of $\mathrm{i}-\mathrm{nc}-\mathrm{TiO}_{2}-\mathrm{o}$ measured using an integrating sphere (a). Changes in absorbance of $\mathrm{i}$-nc- $\mathrm{TiO}_{2}-\mathrm{o}$ with photonic effects in the UV range with respect to 280 -i-nc- $\mathrm{TiO}_{2}-\mathrm{O}$ (b). The stop band positions are labeled.

\subsubsection{Structural Morphology}

Figure 3.11 shows the SEM and STEM images of $\mathrm{i}-\mathrm{nc}-\mathrm{TiO}_{2}-\mathrm{o}$ of different sphere sizes, with the nanocrystallites in the framework of the inverse opal clearly visible at high magnification. Upon calcination at $450{ }^{\circ} \mathrm{C}$ for $4 \mathrm{~h}$, anatase phase was obtained with most crystallites ranging from 6 to $12 \mathrm{~nm}$ in size, as evidenced by the STEM (Fig. 6e and f). The air sphere diameter of $\mathrm{i}-\mathrm{nc}-\mathrm{TiO}_{2}-\mathrm{o}$ is approximately $80 \%$ of the original polystyrene sphere in agreement with $\mathrm{i}-\mathrm{nc}-\mathrm{TiO}_{2}-\mathrm{O}$ previously obtained from alkoxide precursor $^{[27,28]}$, where shrinkage during hydrolysis, condensation and calcination is unavoidable. The topview SEM characterization confirms the high structural ordering of the inverse opals and the absence of a $\mathrm{TiO}_{2}$ overlayer that may impede the slow photon effect. In addition, the morphologies of the nc- $\mathrm{TiO}_{2}$ and crushed-i-nc- $\mathrm{TiO}_{2}-\mathrm{o}$ that serve as the reference in the photodegradation experiment are shown in Fig. 3.12. 

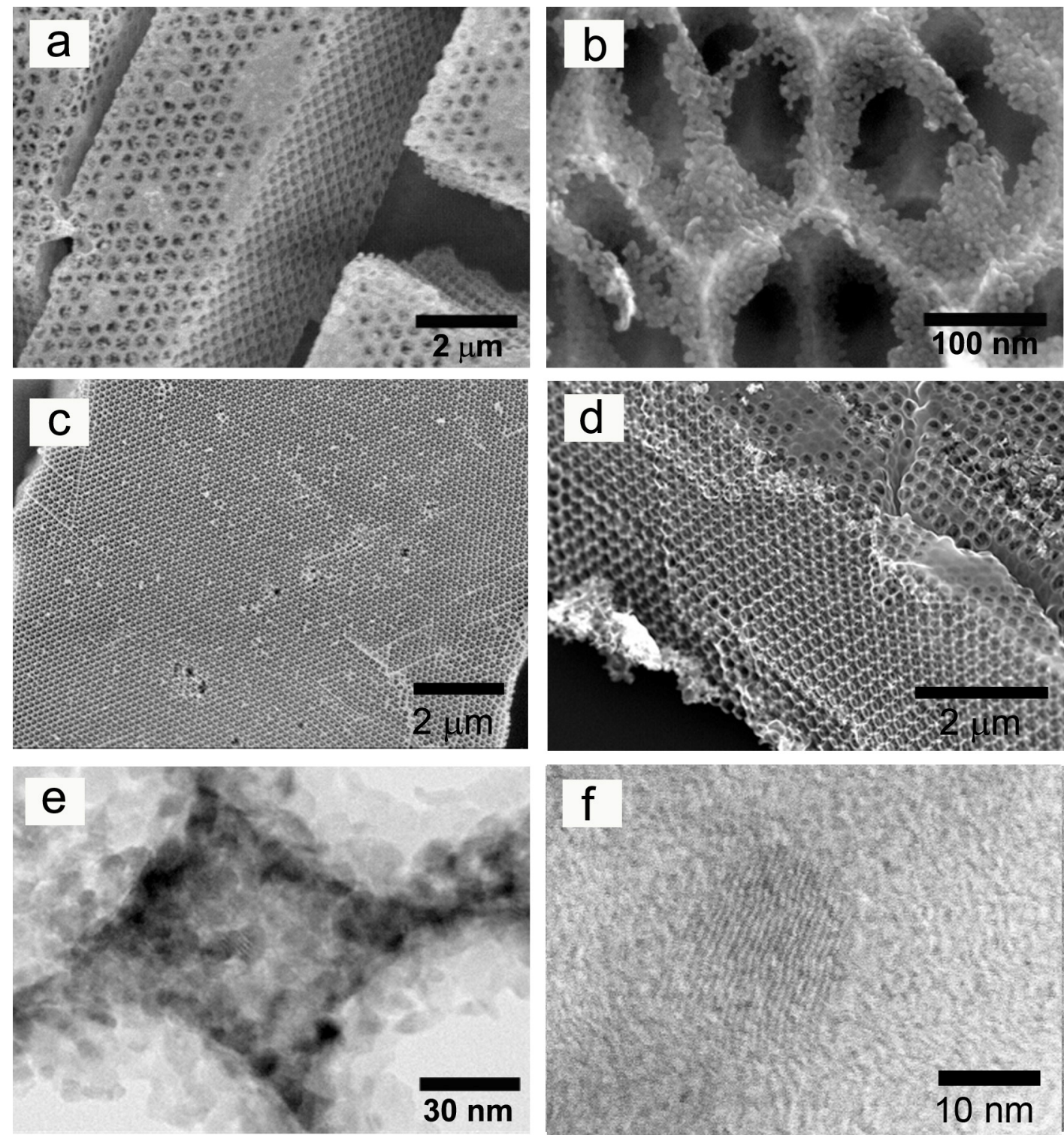

Figure 3.11. SEM images of: $345-\mathrm{i}-\mathrm{nc}-\mathrm{TiO}_{2}$-o on glass substrate as viewed from the top (a); 300i-nc- $\mathrm{TiO}_{2}$-o free-standing domains at different magnifications showing the nanocrystal framework and the long-range ordering ( $b$ and $c$ ); and cross-sectional view of 430-i-nc- $\mathrm{TiO}_{2}-\mathrm{o}(\mathrm{d})$. The nanocrystals with sizes ranging from 6-12 nm (e) and the crystal lattice (f) are seen at higher magnifications under STEM. 

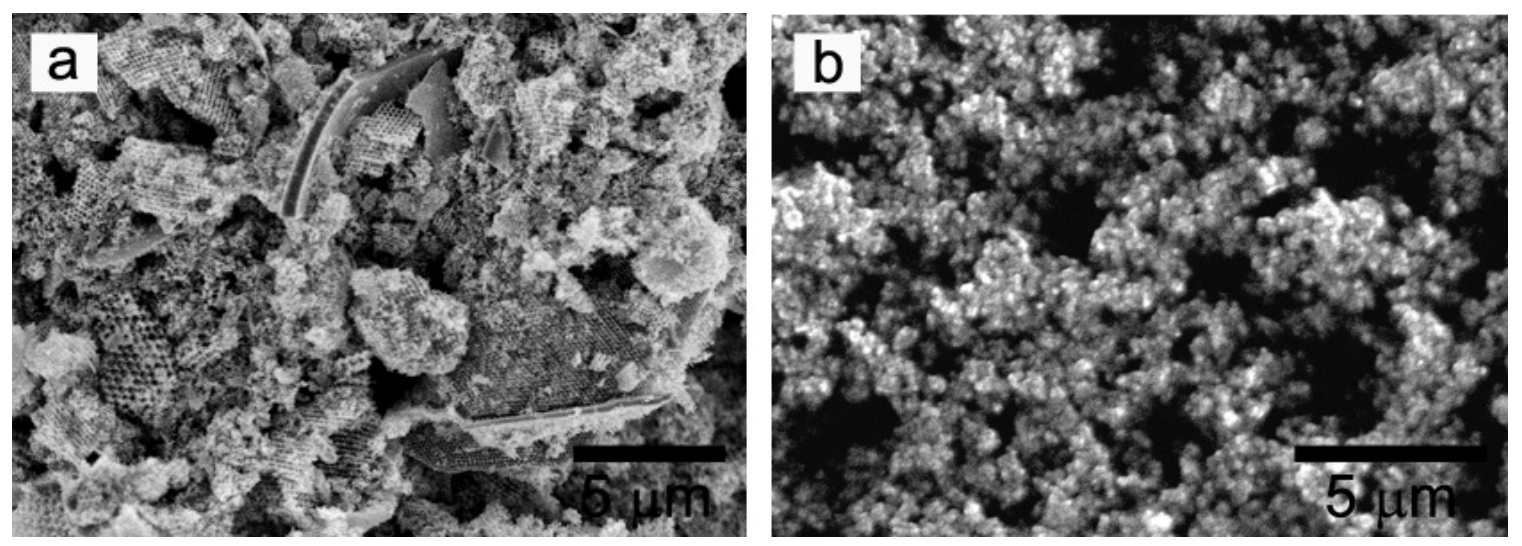

Figure 3.12. $\mathrm{SEM}$ images of crushed-i-nc- $\mathrm{TiO}_{2}-\mathrm{o}$ (a) and $n c-\mathrm{TiO}_{2}$ (b) that were used as references.

\subsubsection{Crystal Phase}

The anatase phase of $\mathrm{i}-\mathrm{nc}-\mathrm{TiO}_{2}-\mathrm{o}$ was confirmed by both XRD and Raman spectroscopy (Fig. 3.13). The Rietveld refinement on XRD data further suggests that the average crystal size is $12 \mathrm{~nm}$, in close agreement with the observations under STEM.
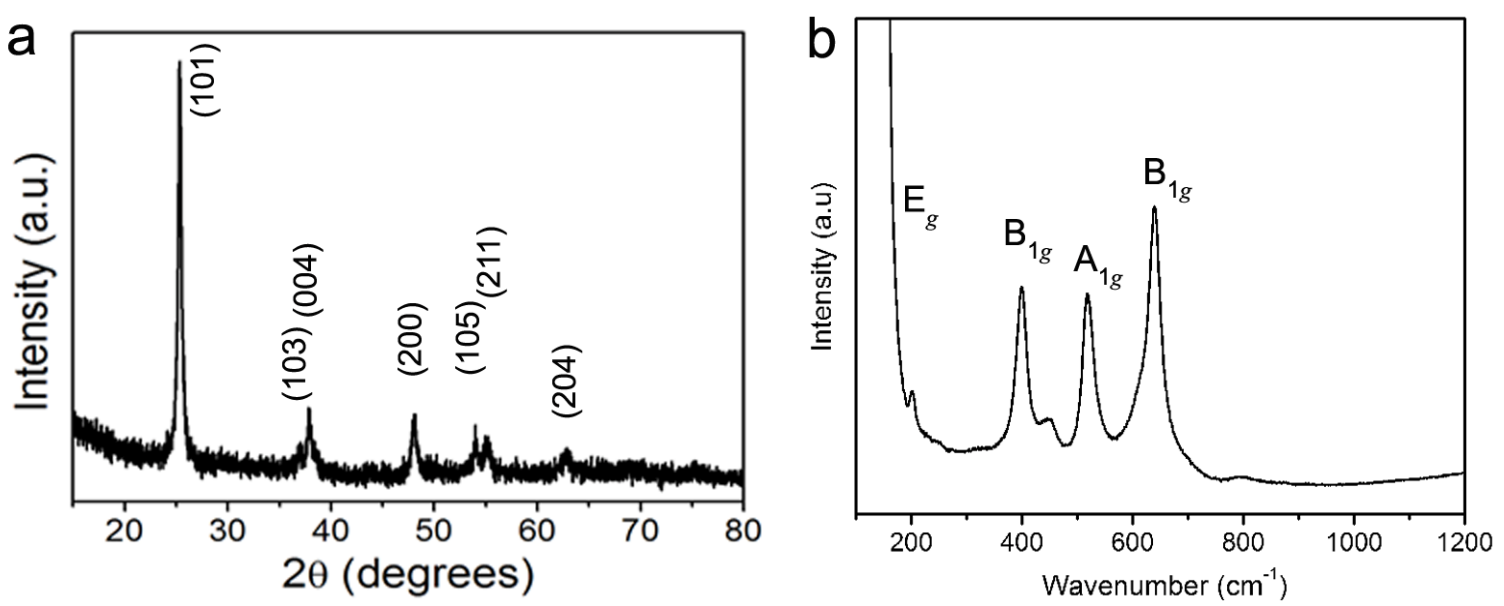

Figure 3.13. Powder XRD (a) and Raman spectrum (b) of i-nc- $\mathrm{TiO}_{2}-\mathrm{o}$ calcined at $450{ }^{\circ} \mathrm{C}$. All peaks correspond to the diffraction and phonon modes of anatase $\mathrm{TiO}_{2}$ respectively. 


\subsection{Solid-State Photodegradation Efficiency}

To demonstrate the influence of the photonic structure on the photo-activity of $\mathrm{TiO}_{2}$, a solid-state photo-degradation experiment with adsorbed methylene blue as probe molecule was employed. The absence of solvent in the experiment ensures the high dielectric contrast of the photonic crystals and eliminates other interfering factors found in a heterogeneous system, such as the diffusion of dye molecules within a highly porous $\mathrm{TiO}_{2}$ inverse opal. The photo-activity of $\mathrm{i}-\mathrm{nc}-\mathrm{TiO}_{2}-\mathrm{o}$ with different photonic stop-band energies is compared to a crushed i-nc- $\mathrm{TiO}_{2}-\mathrm{o}$ film and a conventional nc- $\mathrm{TiO}_{2}$ film. Since modes with slow photons for which the electromagnetic fields are localized in the high dielectric part of the material are found at the red-edge of the stop-band, changing the stop-band position moves the energy of slow photons in-and-out of the anatase electronic absorption. Two key concepts are explored herein: (i) the influence of slow photons on enhancing the absorption and photo-activity of $\mathrm{TiO}_{2}$, examined using monochromatic irradiation, and (ii) the practical use of slow photons in "real world" photo-degradation condition, namely under white light irradiation $(>300 \mathrm{~nm})$. Photoactivity under monochromatic light irradiation at $370 \pm 10 \mathrm{~nm}$ was first examined because the effects of stop-band reflection, slow photons and random light scattering can be independently studied using this narrow irradiation window and i-nc- $\mathrm{TiO}_{2}-\mathrm{o}$ having different stop-band energies. Moreover, the absorption of anatase $\mathrm{TiO}_{2}$ is low in the spectral region of $360-380 \mathrm{~nm}$, so the enhancement there can be readily detected and modeled. White light irradiation, on the other hand, involves a complex interplay of various photonic effects, but through the strategic tuning of the stop-band energy, slow 
photons can dominate over other effects to give an overall enhancement under real world condition.

\subsubsection{Adsorption of Methylene Blue}

Methylene blue is a cationic dye and adsorption on $\mathrm{TiO}_{2}$ is through electrostatic interaction. Therefore only under basic condition would the surface of $\mathrm{TiO}_{2}$ be negatively-charged and methylene blue adsorb. The monomeric form has peak absorbance at $660 \mathrm{~nm}$ while the dimers and multi-molecular aggregates have absorbance blue-shifted at 610 and $\sim 570 \mathrm{~nm}$ due to the $\pi-\pi$ interaction between molecules. ${ }^{[29,30]}$ Hence the nature of the adsorbed dye molecule can be judged by the shape of the absorbance spectra. In order to compare different $\mathrm{i}-\mathrm{nc}-\mathrm{TiO}_{2}-\mathrm{o}$ films, the dye loading was standardized with respect to the surface area (estimated to be $\sim 100 \mathrm{~m}^{2} / \mathrm{g}$, see Chapter 5). Achieving only a monolayer of adsorbed methylene blue on the films was crucial since the photo-degradation rate depends on the coverage of the dye. To determine the concentration of methylene blue required to obtain the monolayer coverage, an adsorption isotherm was measured. Figure 3.14 shows the Langmuir isotherms for the adsorption of methylene blue on all films, in agreement with previous reports. ${ }^{[31]}$ The adsorption isotherms suggest dye coverage approaches saturation (monolayer) at an equilibrium concentration of $7 \mu \mathrm{M}$, above which dye dimerization becomes dominant. At $7 \mu \mathrm{M}$, the absence of multilayer or aggregates of dye molecules further ensures the direct contact of each molecule to the surface of $\mathrm{TiO}_{2}$, giving reproducible photo-degradation kinetics. 


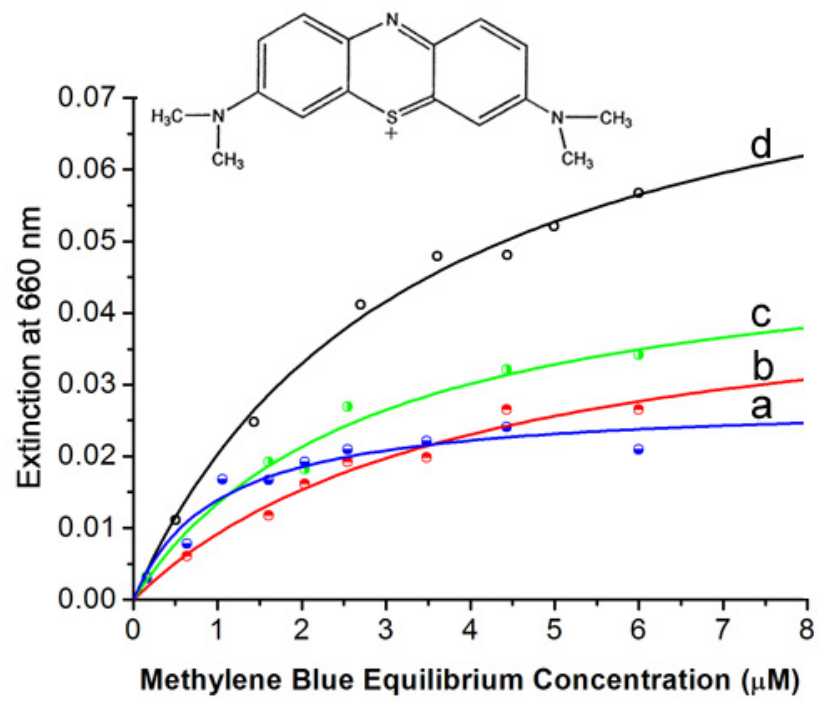

Figure 3.14. Adsorption behavior of methylene blue on $\mathrm{TiO}_{2}$ films plotted as Langmuir isotherms for nanocrystalline $\mathrm{TiO}_{2}$ (a), and inverse opals with photonic stop-band at $345 \mathrm{~nm}(\mathrm{~b}), 370 \mathrm{~nm}$ (c) and $500 \mathrm{~nm}(\mathrm{~d})$. The chemical structure of methylene blue is shown on the top.

\subsubsection{Monochromatic Irradiation}

To elucidate the effect of the photonic crystal structure on the photo-degradation activity of $\mathrm{TiO}_{2}$, the kinetics of methylene blue decomposition under monochromatic irradiation at $370 \pm 10 \mathrm{~nm}$ was first examined. Irradiation in one-minute intervals immediately followed dye adsorption. Changes in the dye extinction spectra with increasing irradiation time are shown in Fig. 3.15a. In general, the dye extinction decreases in intensity and blue-shifts as the decomposed intermediates have less extended $\pi$-orbital system. ${ }^{[32,33]}$ Figure 3.15 b shows a logarithmic plot of the relative extinction as a function of irradiation time for three representative inverse opals and nc- $\mathrm{TiO}_{2}$, confirming the first-order exponential decay of the adsorbed dye molecules. Mesoporous $\mathrm{SiO}_{2}$ was used as the blank, which showed insignificant degradation of methylene blue upon irradiation. The enhancement factor $(\mathrm{EF})$, calculated as the ratio of decay rate 

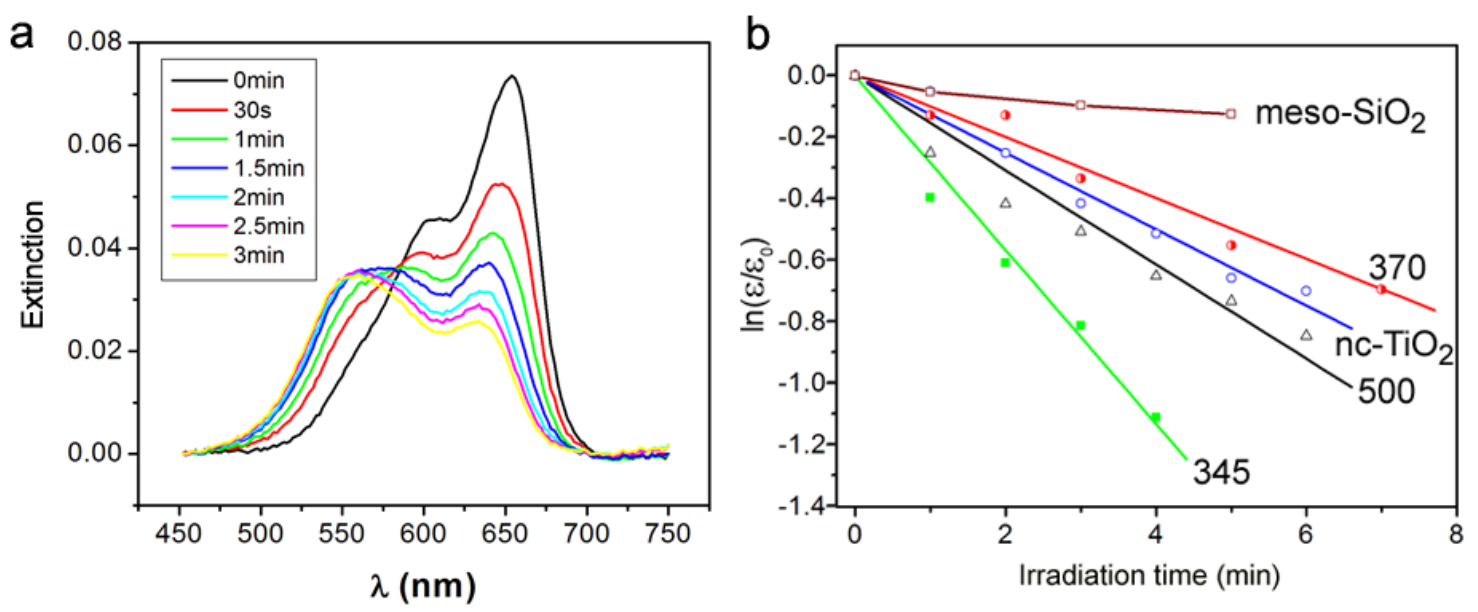

Figure 3.15. Typical extinction spectra of methylene blue during photo-degradation under UV irradiation (a). Logarithmic plot of relative dye extinction (b) showing the first order decay rate for nc- $\mathrm{TiO}_{2}$ and inverse opals with stop-band at 345, 370 and $500 \mathrm{~nm}$. Meso- $\mathrm{SiO}_{2}$ was used as the blank.

constant of $\mathrm{i}-\mathrm{nc}-\mathrm{TiO}_{2}-\mathrm{o}$ to $\mathrm{nc}-\mathrm{TiO}_{2}$ provides a quantitative comparison of the photodegradation. Figure 3.16 shows a plot of enhancement factor vs. stop-band position for $370 \mathrm{~nm}$ irradiation at 0,10 and 20 degrees relative to the normal of the films. When irradiation was normal to the surface ( $0 \mathrm{deg})$, the samples exhibit three distinct scenarios: (i) photonic stop-band in the illumination window, represented by $370-\mathrm{i}-\mathrm{nc}-\mathrm{TiO}_{2}-\mathrm{o}$; (ii) slow photons at $370 \mathrm{~nm}$, found at the red-edge of 345-i-nc- $\mathrm{TiO}_{2}-\mathrm{o}$, and (iii) absence of the photonic effect, represented by 280-, 300-, 325-, 430- and 500-i-nc- $\mathrm{TiO}_{2}-\mathrm{o}$. For i-nc$\mathrm{TiO}_{2}-\mathrm{O}$ that have no photonic effect at $370 \mathrm{~nm}$, the EF are $1-1.2$. The slight increase of photo-activities observed for some samples may originate from random light scattering. This finding points out that the intrinsic properties of the $\mathrm{TiO}_{2}$ crystallites of the inverse opals and conventional nanocrystalline films are similar and that light scattering alone can only account for a small enhancement in the photo-activity. 


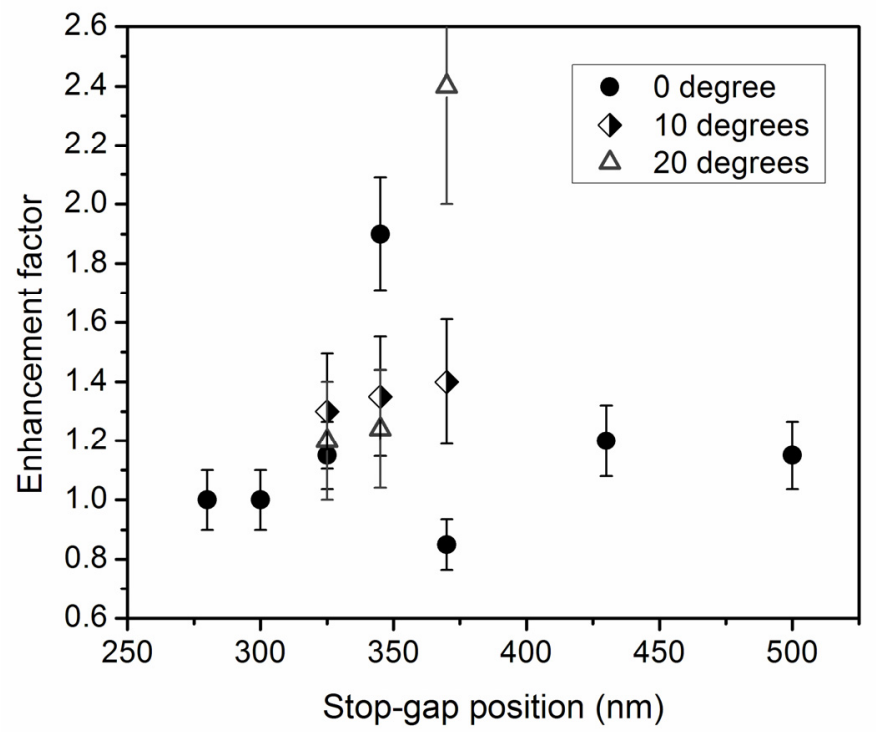

Figure 3.16. Photo-activity enhancement factor for all $\mathrm{i}-\mathrm{nc}-\mathrm{TiO}_{2}-\mathrm{o}$ plotted as a function of stopband energies under the same monochromatic irradiation. Photo-activity with irradiation offnormal at 10 and 20 deg shows strong angle-dependency for 345 - and $370-\mathrm{i}-\mathrm{nc}-\mathrm{TiO}_{2}$-o due to photonic effects near the irradiation wavelength.

The effect of the photonic crystal on the degradation rate is clearly evident for 345- and 370-i-nc- $\mathrm{TiO}_{2}-\mathrm{o}$. When the energy of the photonic stop-band coincides with that of the irradiating light, as is the case for $370-\mathrm{i}-\mathrm{TiO}_{2}-\mathrm{o}$, partial light reflection leads to a lower decay rate constant compared to $n c-\mathrm{TiO}_{2}$. Consequently, the film of $370-\mathrm{i}-\mathrm{TiO}_{2}-\mathrm{o}$ is the least active for the degradation of methylene blue, with an EF of 0.85 . To achieve optically amplified photo-oxidation, the red-edge of the photonic stop-band, where slow photons propagate in the dielectric part of the photonic crystal, needs to be in the illumination window. This requirement is realized in $345-\mathrm{i}-\mathrm{TiO}_{2}-\mathrm{O}$. The decay rate of methylene blue on $345-\mathrm{i}-\mathrm{TiO}_{2}-\mathrm{o}$ is indeed twice faster compared to nc- $\mathrm{TiO}_{2}$, with an $\mathrm{EF}$ of 1.9. It represents the first optically amplified photochemical reaction through the direct coupling of slow photons to $\mathrm{TiO}_{2}$. 
To further support these findings and interpretation, the group velocity of a finite thickness inverse opal was modeled using a SWA approach. Since the group velocity can only be properly defined for a non-absorbing system, the numerical calculations were done for the transparent region of $\mathrm{TiO}_{2}$. The maximum amplification factor ${ }^{[34]}$, calculated as the ratio of the speed of light in a nc- $\mathrm{TiO}_{2}$ film of same thickness to the group velocity of light traveling through the $\mathrm{i}-\mathrm{nc}-\mathrm{TiO}_{2}-\mathrm{o}$, reaches a value of 2.1. This maximum value is attained at the red edge of the stop-band, approximately $0.15 \mathrm{eV}$ away from the stop-band maximum. The largest optical enhancement should therefore be roughly $20 \mathrm{~nm}$ redshifted from the stop-band maximum of $345-\mathrm{i}-\mathrm{nc}-\mathrm{TiO}_{2}-\mathrm{o}$, in good agreement with the experimental observations. Although the applied model is effectively one-dimensional and can only approximate the three-dimensional behavior of the experiments, the results agree almost quantitatively. The twofold enhancement of the decay rate for 345-i-nc$\mathrm{TiO}_{2}$-o can therefore be attributed almost exclusively to slow photons at the photonic crystal stop-band edge.

\subsubsection{Effect of Incidence Angle}

To confirm the origin of the enhancement, angle-dependent photo-degradation was carried out. As photonic properties arise from the interferences of light with crystal planes in the periodic structure, they change depending on the angle of incidence. The fundamental stop band blue-shifts with increasing angle of incidence (from normal of the film). ${ }^{[35]}$ So depending how photodegradation efficiency changes (or not) with different irradiation angle, one can determine if the enhancement originates from photonic properties or isotropic random light scattering. The photo-activities for irradiation at 10 
and 20 deg off-normal for 325-, 345- and 370-i-nc- $\mathrm{TiO}_{2}-\mathrm{o}$ are shown in Fig. 3.16. Increasing the angle of incidence leads to a blue-shift in the stop-band. Consequently, the energy of slow photon for 345-i-nc- $\mathrm{TiO}_{2}$-o moves out of the irradiation window at 10 and $20 \mathrm{deg}$ and causes a significant decrease in its photo-activity. In contrast the EF for 370-inc- $\mathrm{TiO}_{2}$-o increases drastically to 2.4 at 20 deg off-normal. Calculation using modified Bragg's equation (equation 1.3) suggests that the stop-band of this sample shifts from 370 $\mathrm{nm}$ at $0 \mathrm{deg}$ to $350 \mathrm{~nm}$ at $20 \mathrm{deg}$, which according to the SWA is the position for maximum amplification due to slow photons. Therefore, the angle-dependent photoactivity of $\mathrm{i}-\mathrm{nc}-\mathrm{TiO}_{2}$-o strongly supports that the optical enhancement is indeed due to the slow photons. Disordered samples did not show any angle dependence in the EF, as expected (see Chapter 4).

\subsubsection{White Light Irradiation}

After having demonstrated the slow photon enhancement in the monochromatic experiment, the practicality of slow photons in realistic photo-degradation condition needs to be addressed. Which of the two competing effects, slow-photon enhancement or suppressed photo-activity due to higher reflectivity in the stop-band, will dominate under white light irradiation? According to Miguez and co-workers ${ }^{[26]}$ the suppression due to reflectivity always dominates, as the spectral width of the stop-band is argued to be much wider than the region in which enhancement due to slow-photons occurs. On the other hand, it is well known that absorption is able to highly suppress features arising from photonic band structure. ${ }^{[36]}$ If one now designs a system, in which the unwanted reflectivity is suppressed due to high absorption, while at the same time the red edge used 
for enhancement still resides in a sufficiently transparent region, then slow photon enhancement can be expected to dominate. To this end, $\mathrm{TiO}_{2}$ with its long absorption tail is an excellent model system to explore this hypothesis. Samples with stop-bands at 325, 300 and $280 \mathrm{~nm}$ would experience much stronger absorption in the stop-band region than at the red-edge used for slow-photon enhancement, so that absorption by $\mathrm{TiO}_{2}$ effectively decreases the reflection from the photonic structure, as can be seen in Fig. 3.8a.

Figure 3.17 shows the photo-activity of $\mathrm{i}-\mathrm{nc}^{-} \mathrm{TiO}_{2}$-o under white light irradiation as a plot of EF vs. stop-band position. For irradiation normal to the films, an increase in the enhancement factor with the stop-band moving from 370 to $300 \mathrm{~nm}$ was observed as a result of slow photon coupling and the suppression of stop-band reflection. Referring back to the absorption spectra in Fig. 3.10, the higher EF for 300- and 325-i-nc- $\mathrm{TiO}_{2}-\mathrm{O}$ coincide with the enhanced absorption. Therefore, one can conclude that the increased absorption due to slow photons leads to an increase in the photodegradation efficiency. Further blue-shifting of the stop-band to $280 \mathrm{~nm}$ did not yield any enhancement as light with wavelengths $>300 \mathrm{~nm}$ can no longer probe the periodic structure. The $280-\mathrm{i}-\mathrm{nc}-$ $\mathrm{TiO}_{2}-\mathrm{o}$ effectively acted like the conventional nc- $\mathrm{TiO}_{2}$ since both stop-band reflection and slow photons are eliminated by the high absorbance. Furthermore, crushed i-nc- $\mathrm{TiO}_{2}$ o was prepared and its photodegradation activity measured in order to exclude the influence of unwanted effects, which might have arisen due to the treatment of the material during the inversion procedure. The degradation rate for the crushed $\mathrm{i}-\mathrm{nc}-\mathrm{TiO}_{2}-\mathrm{O}$ was within the experimental error and essentially the same as the nc- $\mathrm{TiO}_{2}$ reference film.

As expected, i-nc- $\mathrm{TiO}_{2}-\mathrm{o}$ with stop-band in the absorption edge of $\mathrm{TiO}_{2}(340-400$ $\mathrm{nm})$ have the lowest photoactivity under white light illumination due to the competition 


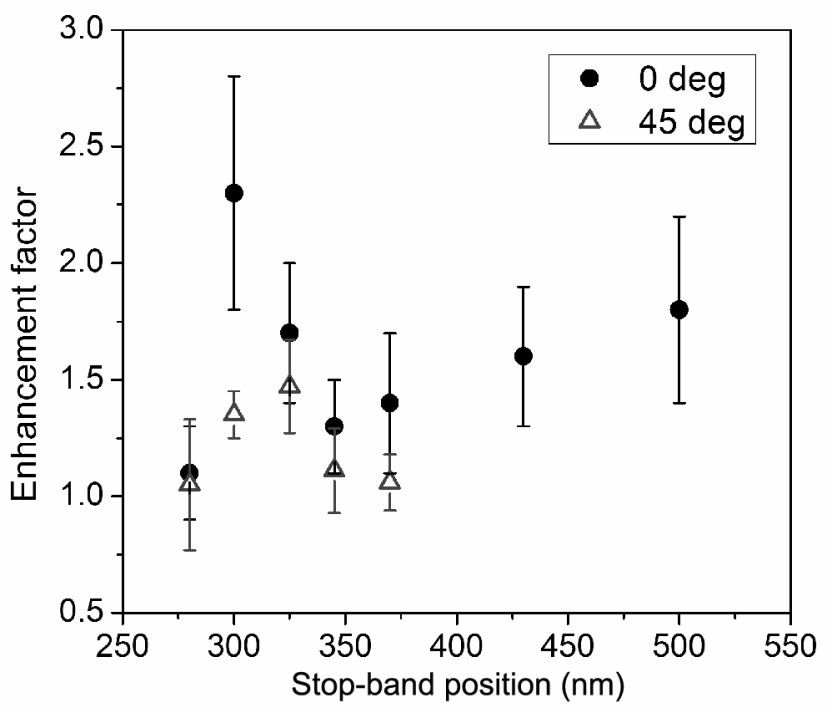

Figure 3.17. Photo-activity enhancement factor for $\mathrm{i}-\mathrm{nc}-\mathrm{TiO}_{2}-\mathrm{o}$ with different stop-band energies, under white light irradiation $(\lambda>300 \mathrm{~nm})$ at 0 and $45 \mathrm{deg}$.

competition between stop-band reflection and slow photons. It is interesting to note here the difference between the monochromatic and the white light experiment: the most effective sample in the monochromatic experiment turned out to be the least effective in the white light experiment, demonstrating the importance of the efficient suppression of reflection. In addition, an enhancement in photo-activity was also found for 430- and 500-i-nc- $\mathrm{TiO}_{2}-\mathrm{O}$. Although the stop-bands are centered away from the absorption of $\mathrm{TiO}_{2}$, the influence of higher order diffraction bands with low group velocity at $300-350 \mathrm{~nm}$ in the photonic structure could have contributed to the faster decay rate. In this case the reflectance of the stop-band plays no role at all, as it is in a spectral region in which $\mathrm{TiO}_{2}$ has no absorption.

Based on the findings, the optimal stop-band position of $\mathrm{i}-\mathrm{nc}-\mathrm{TiO}_{2}$-o for photodegrading adsorbed organic molecules under white light irradiation is $300 \mathrm{~nm}$ with an enhancement factor of 2.3 in comparison to conventional nc- $\mathrm{TiO}_{2}$. To confirm that 
photonic effect is the origin of the enhancement, photo-degradation under irradiation at 45 deg off-normal was investigated for samples with the stop-band in the absorption of $\mathrm{TiO}_{2}$ (Fig. 3.18). All samples except for $280-\mathrm{i}-\mathrm{nc}-\mathrm{TiO}_{2}-\mathrm{o}$ showed different behavior as photonic effects change with angle, whereas multiple scattering effects are expected to be isotropic. The photo-activity of $280-\mathrm{i}-\mathrm{nc}-\mathrm{TiO}_{2}$-o is independent of incident angle since photonic effects play no role in this sample. In comparison to irradiation at normal incidence, the enhancement at $45 \mathrm{deg}$ is much smaller due to the fact that light encounters a less efficient photonic structure. The change in group velocity with respect to the incidence angle is best described by the band diagram. As the samples were rotated along an axis that is perpendicular to the growth direction of the film, the wave vector is moving away from $\Gamma-\mathrm{L}$ to $\Gamma-\mathrm{W}$ in the Brillouin zone. ${ }^{[37]}$ The band diagram for a $f c c$ structure shows little change to the photonic bands near L point (at incidence angle $<20^{\circ}$ ) in L-W zone where the bands remain relatively flat. However, as the angle is increased such that $\mathrm{k}$ increases towards the $\mathrm{W}$ point, the bands are no longer flat, the group velocity increases and the degenerate bands split. Therefore, the slow-photon effect is weaker at $45^{\circ}$ than 10 or $20^{\circ}$. As the stop-band is blue-shifted at $45 \mathrm{deg}$, the most photochemically active inverse opal changes from 300- to 325 i-nc- $\mathrm{TiO}_{2}$-o.

\subsection{Conclusions}

The photo-activity of $\mathrm{TiO}_{2}$ when fashioned as inverse opals was significantly enhanced by slow photons. The energy of the photonic stop-band was optimized with respect to the semiconductor electronic band gap in order to effectively harvest slow photons in the dielectric part of the material, while suppressing unwanted reflectivity 
losses. A twofold increase in the rate of photooxidation of methylene blue by $\mathrm{TiO}_{2}$ was achieved under white light irradiation using inverse opal with stop band at $300 \mathrm{~nm}$.

\subsection{References}

[1] K. Yoshino, S. B. Lee, S. Tatsuhara, Y. Kawagishi, M. Ozaki, A. A. Zakhidov, Appl. Phys. Lett. 1998, 73, 3506.

[2] Y. A. Vlasov, K. Luterova, I. Pelant, B. Honerlage, V. N. Astratov, Appl. Phys. Lett. 1997, 71, 1616.

[3] M. R. Ranade, A. Navrotsky, H. Z. Zhang, J. F. Banfield, S. H. Elder, A. Zaban, P. H. Borse, S. K. Kulkarni, G. S. Doran, H. J. Whitfield, Proc. Natl. Acad. Sci. U. S. A. 2002, 99, 6476 .

[4] N. Daude, C. Gout, C. Jouanin, Phys. Rev. B 1977, 15, 3229.

http://link.aps.org/abstract/PRE/v15/p3229

[5] M. A. Fox, M. T. Dulay, Chem. Rev. 1993, 93, 341.

[6] M. R. Hoffmann, S. T. Martin, W. Y. Choi, D. W. Bahnemann, Chem. Rev. 1995, $95,69$.

[7] A. Emeline, A. Salinaro, N. Serpone, J. Phys. Chem. B 2000, 104, 11202.

[8] A. Salinaro, A. V. Emeline, J. C. Zhao, H. Hidaka, V. K. Ryabchuk, N. Serpone, Pure Appl. Chem. 1999, 71, 321.

[9] A. L. Linsebigler, G. Lu, J. T. Yates Jr, Chem. Rev. 1995, 95, 735.

[10] A. R. Nicolaescu, O. Wiest, P. V. Kamat, J. Phys. Chem. A 2005, 109, 2822.

[11] L. Z. Sun, J. R. Bolton, J. Phys. Chem. 1996, 100, 4127. 
[12] D. W. Bahnemann, M. Hilgendorff, R. Memming, J. Phys. Chem. B 1997, 101, 4265.

[13] D. C. Hurum, A. G. Agrios, K. A. Gray, T. Rajh, M. C. Thurnauer, J. Phys. Chem. $B$ 2003, 107, 4545 .

[14] F. M. Vichi, M. I. Tejedor-Tejedor, M. A. Anderson, Chem. Mater. 2000, 12, 1762.

[15] K. Bourikas, M. Stylidi, D. I. Kondarides, X. E. Verykios, Langmuir 2005, 21, 9222.

[16] R. Asahi, T. Morikawa, T. Ohwaki, K. Aoki, Y. Taga, Science 2001, 293, 269.

[17] Q. Li, J. K. Shang, J. Am. Ceram. Soc. 2008, 91, 660.

[18] T. Ohno, T. Mitsui, M. Matsumura, Chem. Lett. 2003, 32, 364.

[19] Y. Sakatani, D. Grosso, L. Nicole, C. Boissiere, G. J. D. A. A. Soler-Illia, C. Sanchez, J. Mater. Chem. 2006, 16, 77.

[20] V. F. Stone Jr, R. J. Davis, Chem. Mater. 1998, 10, 1468.

[21] J. C. Yu, X. Wang, X. Fu, Chem. Mater. 2004, 16, 1523.

[22] G. R. Bamwenda, S. Tsubota, T. Nakamura, M. Haruta, J. Photochem. Photobiol. A: Chem. 1995, 89, 177.

[23] G. R. Bamwenda, S. Tsubota, T. Nakamura, M. Haruta, Catal. Lett. 1997, 44, 83.

[24] F. Ramiro-Manzano, P. Atienzar, I. Rodriguez, F. Meseguer, H. Garcia, A. Corma, Chem. Comm. 2007, 242.

[25] S. Hore, P. Nitz, C. Vetter, C. Prahl, M. Niggemann, R. Kern, Chem. Comm. 2005, 2011.

[26] A. Mihi, H. Miguez, J. Phys. Chem. B 2005, 109, 15968. 
[27] R. C. Schroden, M. Al-Daous, C. F. Blanford, A. Stein, Chem. Mater. 2002, 14, 3305.

[28] J. E. G. J. Wijnhoven, W. L. Vos, Science 1998, 281, 802.

[29] S. M. Ohline, S. Lee, S. Williams, C. Chang, Chem. Phys. Lett. 2001, 346, 9.

[30] E. Braswell, J. Phys. Chem. 1968, 72, 2477.

[31] R. W. Matthews, J. Chem. Soc., Faraday Trans. 1 1989, 85, 1291.

[32] H. Gnaser, M. R. Savina, W. F. Calaway, C. E. Tripa, I. V. Veryovkin, M. J. Pellin, Int. J. Mass Spectrom. 2005, 245, 61.

[33] A. Houas, H. Lachheb, M. Ksibi, E. Elaloui, C. Guillard, J. M. Herrmann, Appl. Cat. B 2001, 31, 145.

[34] N. A. R. Bhat, J. E. Sipe, Phys. Rev. E 2001, 64, 056604.

[35] H. Miguez, V. Kitaev, G. A. Ozin, Appl. Phys. Lett. 2004, 84, 1239.

[36] G. von Freymann, S. John, M. Schulz-Dobrick, E. Vekris, N. Tetreault, S. Wong, V. Kitaev, G. A. Ozin, Appl. Phys. Lett. 2004, 84, 224.

[37] J. F. Galisteo-Lopez, E. Palacios-Lidon, E. Castillo-Martinez, C. Lopez, Phys. Rev. B 2003, 68, 115109. 


\title{
CHAPTER 4
}

\section{Effect of Disorder on the Photodegradation Efficiency of Inverse Titania}

\section{Opals}

(Reproduced in part with permission from J. Am. Chem. Soc. 2007, 129, 1196. Copyright 2007 American Chemical Society)

\begin{abstract}
In Chapter 3, optically amplified photochemistry with slow photons has been realized when a photoactive material like $\mathrm{TiO}_{2}$ was molded into a photonic crystal and the corresponding energy of photonic bands overlaps with the electronic excitation. While numerous applications of photonic crystals have been proposed, the real practicality depends on the extent of structural imperfection that can be tolerated before significant deterioration in the optical response deems it unrealistic to use. As a result, it is important to evaluate the amount of structural disorder that can be tolerated in inverse $\mathrm{TiO}_{2}$ opals if they are to be used as amplified photocatalysts for photolytic degradation of organics in environmental remediation. This chapter presents a systematic study on the effect of disorder with relation to the photodegradation efficiency $\mathrm{i}$-nc- $\mathrm{TiO}_{2}-\mathrm{O}$, where controlled disorder is achieved by introducing different fractions and sizes of guest spheres into the opal template. The results show that half of the enhancement originally achieved by the inverse opal made from monodisperse $150 \mathrm{~nm}$ spheres is conserved when the domain size of the host spheres remains above a critical threshold. The substitution fraction can be as high as 0.4 when the guest spheres are 1.2 times larger than the host
\end{abstract}


spheres. Such a high tolerance to structural disorder provides strong support for the potential use of inverse $\mathrm{TiO}_{2}$ opals in environmental cleanup applications.

\subsection{Introduction}

The success in the development of photonic crystals in the last decade suggests their indispensable role in a wide range of fields, from optical computing and telecommunication $^{[1]}$ to photovoltaics. ${ }^{[2-4]}$ The optical response of photonic crystals is prone to any structural disorder which is intrinsic in all fabrication methods. In selfassembled opal, a high monodispersity of the spheres is the key to achieving good optical response. ${ }^{[5,6]}$ However, the presence of intrinsic defects such as stacking faults and dislocations is inherent and unavoidable (examples shown in Fig. 4.1). Through detailed confocal and SEM imaging, Vekris et al. have determined that such intrinsic defects arise due to individual spheres that are minutely larger or smaller than the others. ${ }^{[7]}$ However, they found that at very low "impurity" level, the stacking faults merely affect high order photonic bands and not the fundamental stop band. To understand the effect of disorder on the photonic stop band, theoretical modeling of two-dimensional photonic crystal comprised of cylinders with deviation in either lattice position or size has been investigated by Albert et al. ${ }^{[8,9]}$ It was found that low amounts of disorder introduces states near the upper and lower edges of the photonic stop band, leading to a narrowing of the energy range of light attenuation. When the amount of disorder becomes large, defect states in the center of the stop band are introduced and the transmission dip arising from the photonic stop band broadens and becomes asymmetric in shape. From the theoretical modeling, a threshold-like behavior in the amount of disorder was observed, above which 
the optical quality of photonic crystal rapidly decreases. They concluded that the propagation direction of photonic states can further change as a result of disorder.
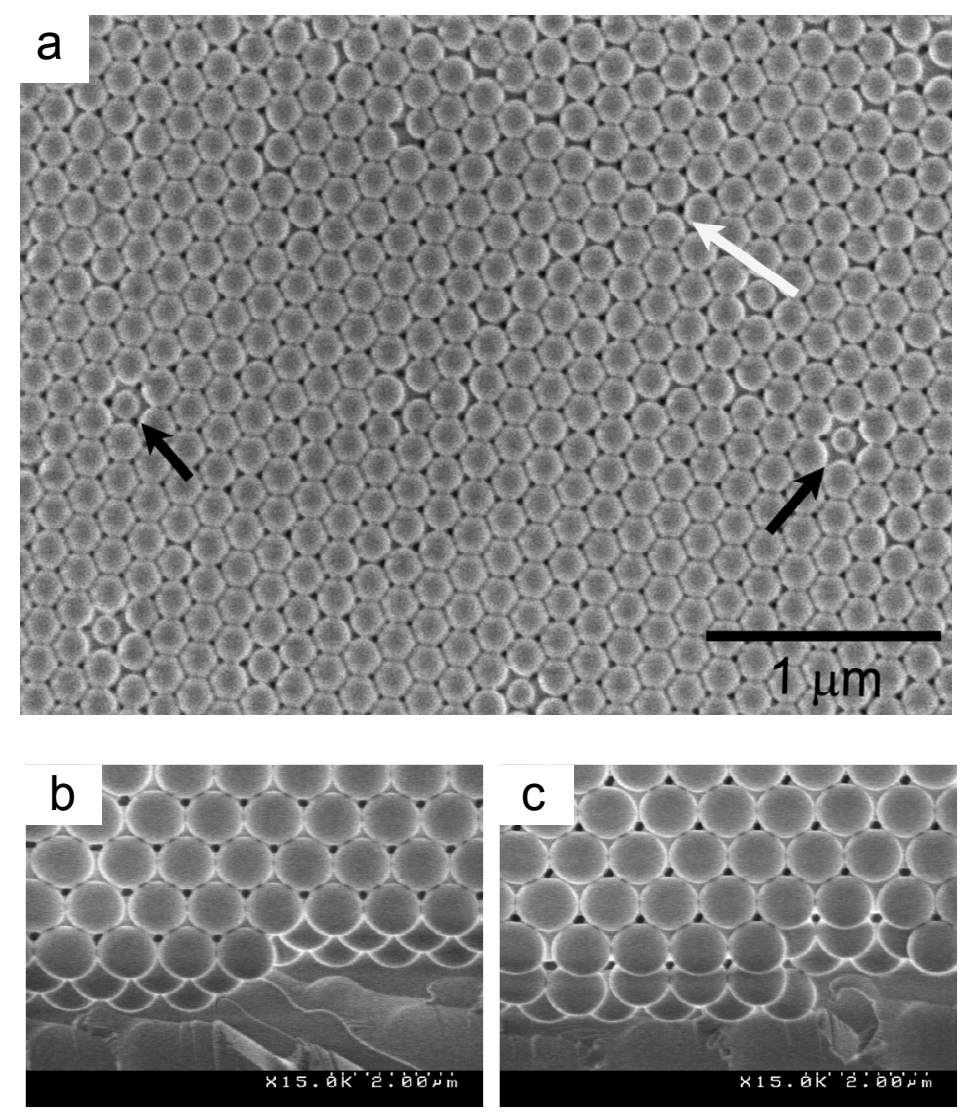

Figure 4.1. Intrinsic defects in colloidal crystal: (a) point defects due to small spheres (black arrow); dislocation (along direction of white arrow) and stacking faults where (b) shows the normal fcc packing of $a b c$ while (c) shows stacking order of $a b a$ of a three-layered opal. (images (b) and (c) reprinted with permission from ref. [7]). Copyright Wiley-VCH.

Work on disorder in 3D photonic crystal has also been active, with most findings obtained from experimental results rather than theoretical calculation due to the massive computer resource required for 3D structures, though this feat has recently been achieved. ${ }^{[10]}$ Experimentally, various researchers have explored the dependence of the 
optical properties of colloidal crystals on the polydispersity of spheres ${ }^{[6]}$ or upon doping with different sizes and amounts of guest spheres. ${ }^{[11-13]}$ In the former case, several studies have concluded that a polydispersity of 6-8 \% causes significant deterioration in the quality and optical response of the colloidal crystal ${ }^{[6,14]}$ while in the latter, both the concentration and size of the guest spheres affect the optical properties and the degree of structural disorder (Fig. 4.2a). In addition, doping the host opal with smaller or larger spheres introduce different defect modes. Adding dielectric material creates donor defect modes while removing dielectric material creates acceptor defect modes (Fig. 4.2b), much like a donor or acceptor atom in semiconductors. ${ }^{[15]}$ The donor modes have their origin at the bottom of the air band (analogous to the conduction band for electrons) while the acceptor modes originate at the top of the dielectric band (analogous to the valence band). These defect modes, in particular the acceptor mode, can act as microresonator cavities that may be useful for lasing applications. To interpret the structural formation of the binary colloidal systems, analogy to atomic alloying has been drawn. X-ray diffraction theory has further been applied to explain the changes in domain size with increasing structural order brought on by the doping spheres. ${ }^{[16]}$ While these studies contribute to the fundamental understanding of the effect of disorder on the optical properties, the actual impact of structural imperfection on a practical application of photonic crystals remains unexplored. Herein the first systematic study of the effect of disorder on the optical properties and the slow-photon enhanced photodegradation efficiency of $\mathrm{i}-\mathrm{nc}-\mathrm{TiO}_{2}-\mathrm{o}$ is presented. The potential real-life performance of inverse titania opal catalysts made from sub-micron spheres with limited monodispersity is addressed. 

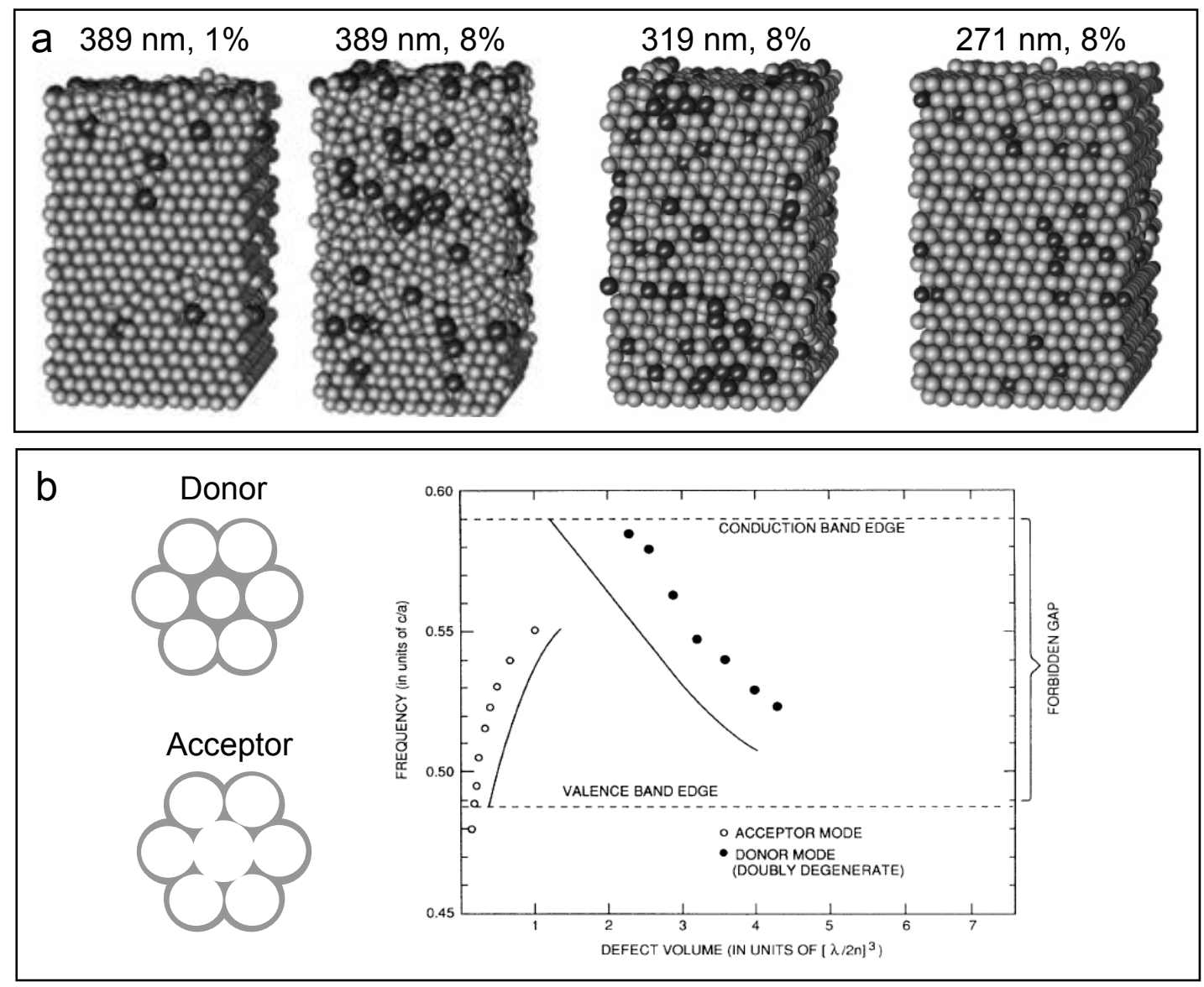

Figure 4.2. (a) Computer simulated images of guest-host colloidal crystal with $295 \mathrm{~nm}$ host spheres and guest spheres of different sizes at different concentrations (as labeled in a). (b) Schematic of donor and acceptor defect modes in a colloidal system where the dielectric material is in grey, and a plot of the defect mode frequencies as a function of defect volume. The acceptor mode starts at the lower energy side of the photonic bandgap while the donor mode appears at the high energy side. (images reprinted with permission from ref. [12] and [15]). Copyright American Chemical Society and American Physical Society.

\subsection{Experimental Methods}

Growth of binary opal template and subsequent infiltration with $\mathrm{Ti}(\mathrm{OBut})_{4}$ were performed using the same techniques as outlined in Chapter 3. Spheres of 130, 150, 180, 
210 and $240 \mathrm{~nm}$ in diameter were used to produce the binary template. The sphere solutions were mixed in ethanol with the total volume fraction of the spheres kept constant at $0.5 \mathrm{vol} \%$. Due to the less efficient packing in the binary system, the concentration of $\mathrm{Ti}(\mathrm{OBut})_{4}$ was increased to $0.7-1.2 \mathrm{vol} \%$.

Solid-state photodegradation experiment was carried as previously mentioned using only white light $\left(40 \mathrm{~mW} / \mathrm{cm}^{2}\right)$ as irradiation source. Both $\mathrm{nc}-\mathrm{TiO}_{2}$ and crushed-inc- $\mathrm{TiO}_{2}-\mathrm{o}$ were used as references.

\subsection{Characterization of Binary Polystyrene Opal and Inverse $\mathrm{TiO}_{2}$ Opals}

In Chapter 3, i-nc- $\mathrm{TiO}_{2}$-o with stop-band position at $300 \mathrm{~nm}$ proved to be the most efficient photocatalyst owing to the slow-photon enhancement and the suppression of stop-band reflection. The template used in this case was $150 \mathrm{~nm}$ polystyrene spheres. Since the enhancement comes from the photonic structure, one would anticipate its effect to diminish with increasing structural disorder. Hence a series of experiments was devised, where the disorder was introduced by substituting different fractions of $150 \mathrm{~nm}$ spheres with different sphere sizes such as $180 \mathrm{~nm}$ or $210 \mathrm{~nm}$. The binary system of 150 $\mathrm{nm}$ and $180 \mathrm{~nm}$ spheres is denoted as $(1-x) 150-x 180$ where $x$ is the mole fraction of 180 nm spheres (other binary systems follow the same notation but with different sphere sizes). The suffix $\mathrm{i}-\mathrm{nc}-\mathrm{TiO}_{2}-\mathrm{o}$ is appended when the structure is the inverse opal. For simplicity, the size of the spherical voids in the inverse structure is considered the same as the template sphere size; the voids are actually $\sim 80 \%$ of the original template size as a result of precursor polymerization-induced shrinkage during the inversion process. This chapter first presents the optical properties of both the opal template and inverse structure 
of (1-x)150-x180 and (1-x)150-x210 systems with the focus on the effect of disorder on photonic strengths. The trend in the photocatalytic efficiency of the binary i-nc- $\mathrm{TiO}_{2}-\mathrm{o}$ is then discussed in relation to the optical data. Lastly, other binary systems, such as 130180 , are investigated to address the effect of light scattering.

\subsubsection{Optical Properties of Binary Polystyrene Opal Templates}

The optical spectra of (1-x)150-x180 templates show Bragg diffraction for all compositions, as seen in Fig. 4.3a. With increasing fraction of $180 \mathrm{~nm}$ spheres, the stopband position red-shifts and the transmission dips become weaker, broader and skewed to longer wavelengths, consistent with observations of previous research. A plot of stopband position as a function of $x$ shows deviation from linear dependency (Fig. 4.3c). At

small $x$, the stop-band position remains relatively constant while the transmission dip decreases in magnitude. The optical spectra of (1-x)150-x180 templates feature stop bands for all compositions, in close agreement with work done by Lopez and co-workers, in which they reported a decrease in stop-band reflection by half when 0.2 fraction of 350 $\mathrm{nm}$ spheres were incorporated into the $270-\mathrm{nm}$ host template. For small spheres under investigation $(<200 \mathrm{~nm})$, the packing may be even more tolerant to variations in particle size as a result of the hydration layer around the highly-charged polystyrene spheres. Hence photonic properties remain intact for all $x$ in $(1-x) 150-x 180$. On the contrary, the optical spectra of (1-x)150-x210, seen in Fig. 4.3b, suggest a much higher degree of disorder than (1-x)150-x180. As $210 \mathrm{~nm}$ spheres are much larger than $150 \mathrm{~nm}$, significant amount of disorder is introduced and even at very small $x$, stop-band diffraction vanishes. 

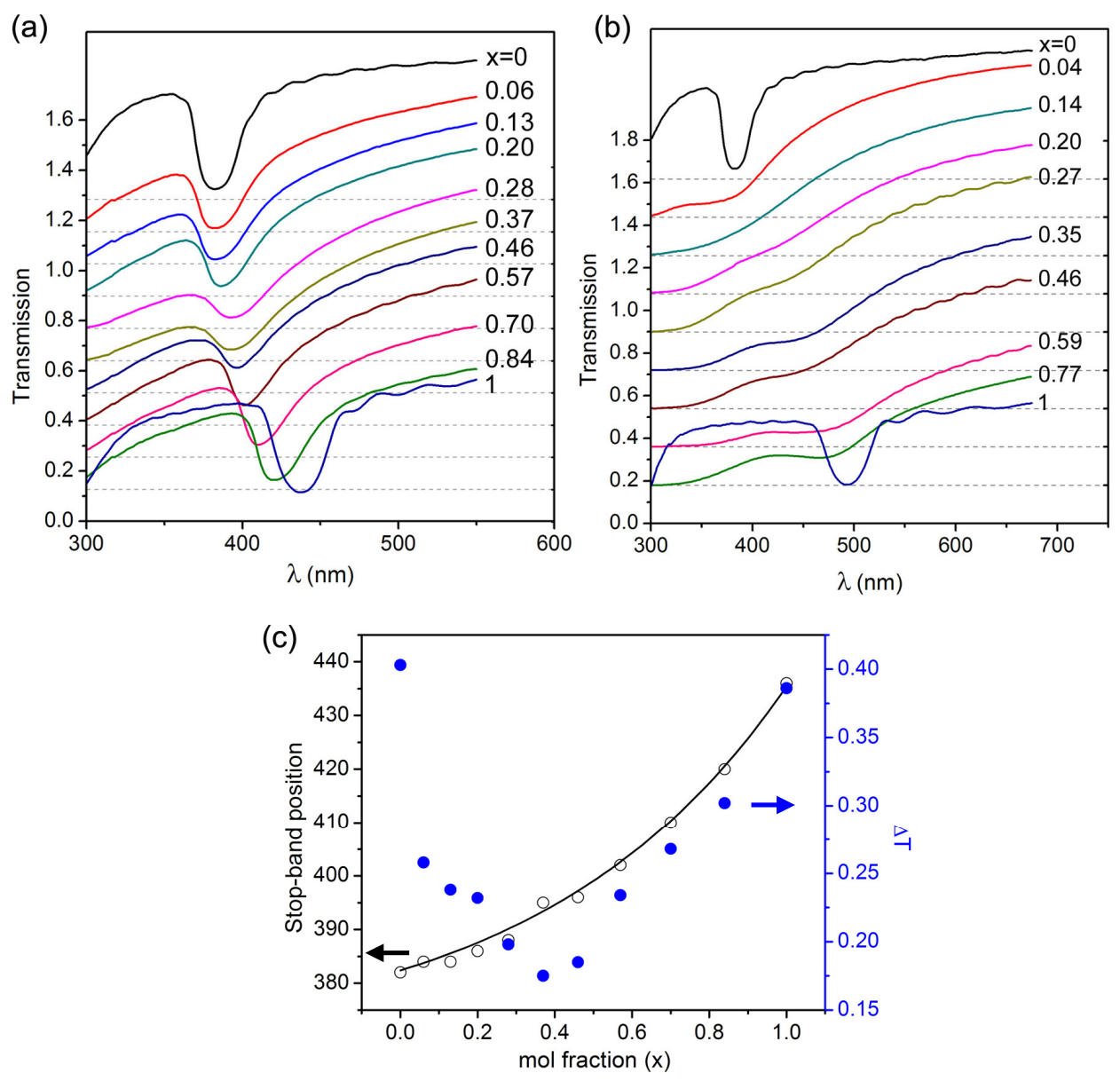

Figure 4.3. Transmission spectra of (1-x)150-x180 (a) and (1-x)150-x210 (b) polystyrene opal templates, and trends in stop-band position and the transmission difference $\left(T_{\text {background }}-T_{\min }\right.$ at midgap) as a function of $x$ for (1-x)150-x180 opal templates (c). The transmission spectra are displaced vertically for clarity with the dashed lines marking the 0 transmission level for each spectrum.

\subsubsection{Domain Size of (1-x)150-x180 Opal Template}

The decrease in light attenuation of (1-x)150-x180 shown in Fig. 4.3a can be explained in terms of the decreasing domain size, as reported by Gates and Xia. ${ }^{[1]}$ In their study, the optical properties of opals $(230 \mathrm{~nm})$ doped with smaller spheres $(150 \mathrm{~nm})$ were found to correlate with equations of dynamic X-ray diffraction and light scattering 
derived by Spry and Koshan. ${ }^{[16]}$ The derivation shows that the transmittance at midgap ( $T$ ) depends on the "effective" thickness of the (111) packing $(t)$ according to the following:

$$
T_{\lambda}=\left[\cosh \left(t / t_{0}\right)\right]^{-2}
$$

and

$$
t_{0}=\left[w_{y}\left(\lambda_{\min }\right)^{2} \sin \theta\right] /[\pi(\Delta \lambda)]
$$

where $\lambda_{\min }$ is the wavelength of transmittance minima, $\theta$ the angle between incident light and (111) planes (assumed to be $90^{\circ}$ ) and $\Delta \lambda$ the bandwidth of the stop band, taken as the full width at half maximum. The numerical factor, $w_{y}$, was determined as 0.723 in our (1$x$ )150-x180 system by solving the equation using known $t$ for $x=0$ and $x=1$. Due to significant Rayleigh scattering below $400 \mathrm{~nm}$, the transmission of all compositions have been corrected to that of $x=0$ for calculation. Figure 4.4 shows the effective number of (111) layers of (1-x)150-x180 as a function of $x$ assuming the diffraction originates from perfect closed-packing of either $150 \mathrm{~nm}$ or $180 \mathrm{~nm}$ domains. The number of (111) layers in the domain drastically decreases from 21 to 13 with the incorporation of just $6 \mathrm{~mol} \%$ of $180 \mathrm{~nm}$ spheres. The domain size however decreases only slightly upon further doping $(0.1<x<0.4)$ to a minimum of 9 layers. It should be noted that an accurate determination of the number of (111) layers for $0.4<x<0.6$ is not feasible as one must assume an inter-planar distance for calculating the number of layers from $t$. It is difficult to judge whether the diffraction for $0.4<x<0.6$ arises from $150 \mathrm{~nm}$ or $180 \mathrm{~nm}$ domains, or possibly from unit cells comprised of the two spheres. As a result, the domain size is best approximated for compositions near the end members. 


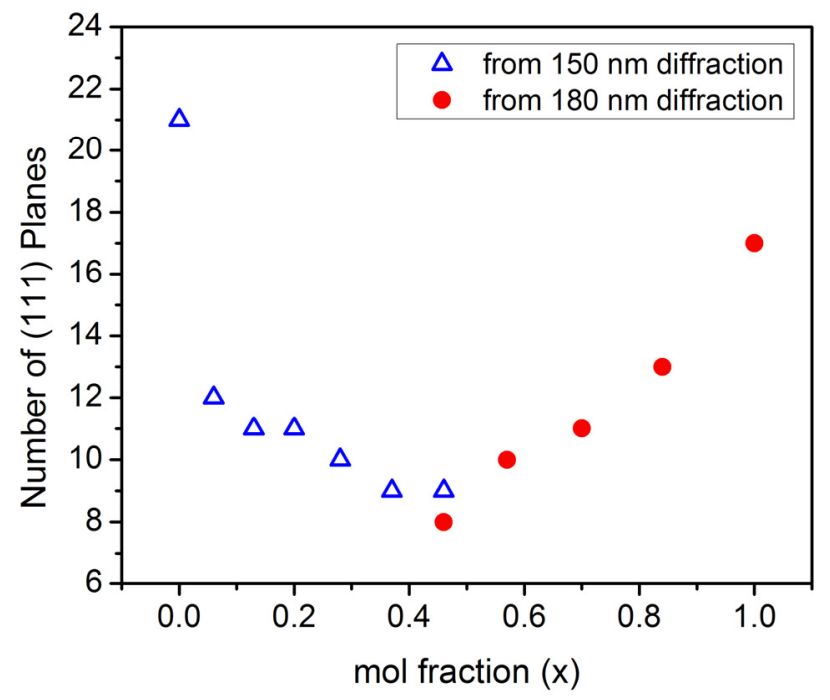

Figure 4.4. The relationship between the number of (111) planes and composition in $(1-x) 150$ $x 180$-i-nc- $\mathrm{TiO}_{2}-\mathrm{o}$, assuming that the diffraction comes from (111) planes of closed-packing of either $150 \mathrm{~nm}$ or $180 \mathrm{~nm}$ spheres.

Data on the effective thickness show a more gradual decrease for $180 \mathrm{~nm}$ spheres doped with $150 \mathrm{~nm}$ ( $x$ closer to 1$)$ than $150 \mathrm{~nm}$ doped with $180 \mathrm{~nm}$ ( $x$ closer to 0$)$. Doping with larger spheres introduces defects that destroy long-range order much more significantly than doping with smaller spheres. Paquet et al. noted that large-host spheres form a cage-like structure around small-guest spheres without causing much change in the lattice at low doping concentrations. ${ }^{[12]}$ A smaller sphere effectively occupies a position of a larger host sphere in the lattice increasing the void space and decreasing the overall refractive index of the template. In this case, substitution with smaller spheres does not change the lattice dimension significantly as the physical contact between the larger spheres governs the unit cell lattice. The domains giving rise to (111) diffraction from $180 \mathrm{~nm}$ sphere-packing may well contain $150 \mathrm{~nm}$ spheres as point defects so that the domain size decreases more gradually. Therefore for compositions going away from $x$ 
$=1$, the void space increases leading to the blue-shift of the stop band and a decrease in the magnitude of the transmission dip (Fig. 4.3a).

\subsubsection{Optical Properties of Binary Inverse Titania Opals}

While the optical response of the template is easily monitored, that of the inverse structure is difficult to observe due to the strong absorption of anatase $\mathrm{TiO}_{2}$ at $300-350$ $\mathrm{nm}$. Indeed none of the compositions other than $x=1$ features stop-band reflection in air. However, in Chapter 3 it was shown that the stop-band reflection is more distinguishable when the samples are immersed in water. By increasing the overall refractive index, the stop band red-shifts to the more transparent region of $\mathrm{TiO}_{2}$. Figure $4.5 \mathrm{a}$ shows the reflectance spectra of $(1-x) 150-x 180-\mathrm{i}-\mathrm{nc}-\mathrm{TiO}_{2}-\mathrm{O}$ in water. For $x=0$, the stop-band maximum in water is at $325 \mathrm{~nm}$. It diminishes in intensity at $x=0.06$ and becomes undetectable for $0.13<x<0.57$. At the other end of the composition range, the stop-band red-shifts when $x$ decreases from 1 . The reflectance maximum moves from 360 to 364 to 370 for $x=1,0.84$ and 0.70 , respectively. The red-shift in the stop-band position of the inverse structure is expected considering the void space in the original template increases when $150 \mathrm{~nm}$ spheres substitute host spheres in the 180-nm lattice. Therefore the inverse structure with partial disorder, e.g. $x=0.84$, has a higher filling fraction of $\mathrm{TiO}_{2}$ than one with monodisperse spherical voids, e.g. $x=1$. As the amount of disorder increases, the stop band of the inverse structure vanishes much more quickly than that of the template due to the difficulty in detecting photonic structure at the absorption edge of $\mathrm{TiO}_{2}$ and the fact that structural defects and disorder may be accentuated in the inverse structure as a result of the shrinking of $\mathrm{TiO}_{2}$ precursor. Figure $4.5 \mathrm{~b}$ shows that no stop-band reflection 
could be detected for any disordered $(1-x) 150-x 210-\mathrm{i}-\mathrm{nc}-\mathrm{TiO}_{2}-\mathrm{o}$ in air or water, supporting the higher extent of disorder caused by the much larger $210 \mathrm{~nm}$ spheres.
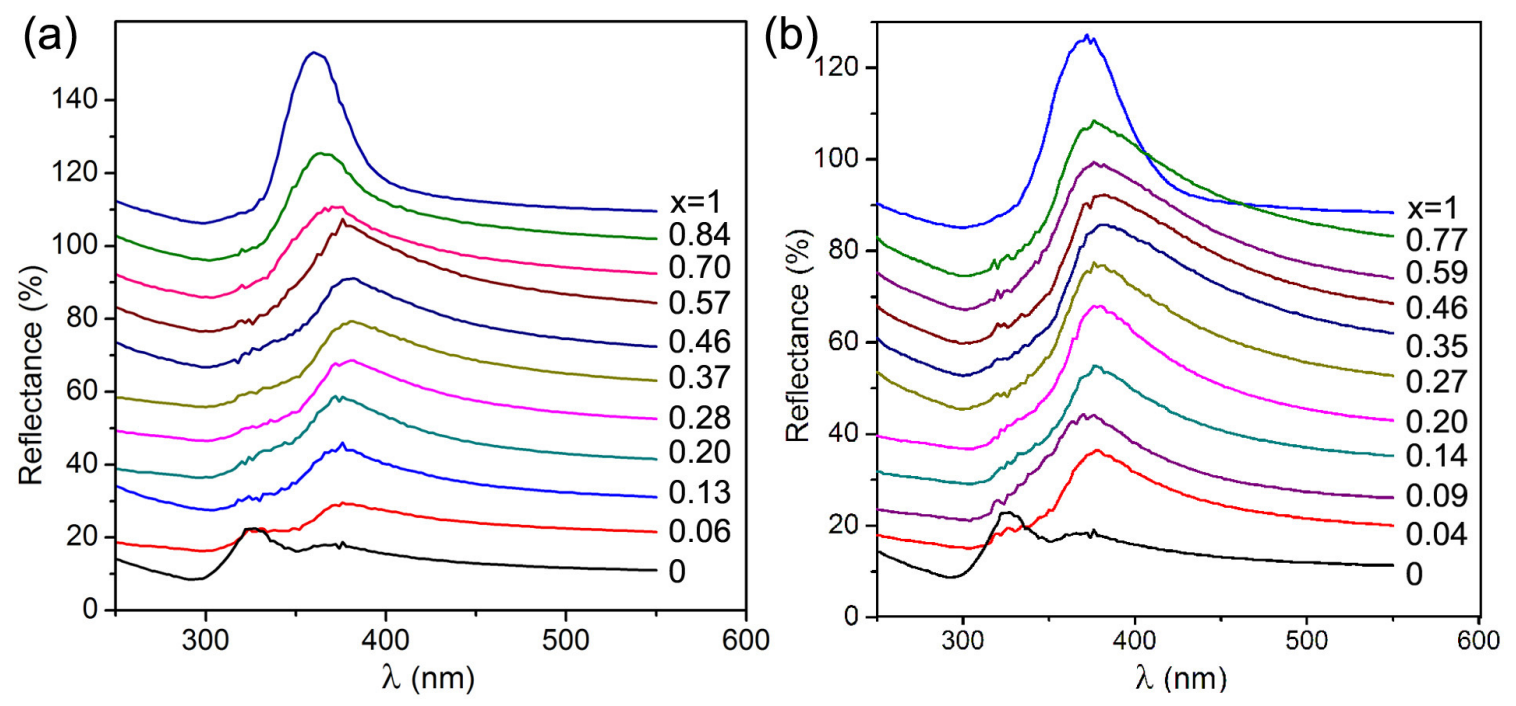

Figure 4.5. Reflectance spectra of (1-x)150-x180-i-nc- $\mathrm{TiO}_{2}$-o (a) and (1-x)150-x210-i-nc- $\mathrm{TiO}_{2}-\mathrm{o}$ (b) in water. Spectra are displaced vertically for clarity.

As diffuse scattering is significant in $\mathrm{i}-\mathrm{nc}-\mathrm{TiO}_{2}-\mathrm{O}$ especially in disordered samples, which visibly are more opaque than ordered $\mathrm{i}-\mathrm{nc}-\mathrm{TiO}_{2}-\mathrm{o}$, real absorbance of the film cannot be deduced from the transmission spectra. As shown in Chapter 3, the true absorbance can be measured using an integrating sphere. Figure $4.6 \mathrm{a}$ and $\mathrm{b}$ show the absorption spectra of $(1-x) 150-x 180-\mathrm{i}-\mathrm{nc}^{-\mathrm{TiO}_{2}-\mathrm{o}}$ and $(1-x) 150-x 210-\mathrm{i}-\mathrm{nc}-\mathrm{TiO}_{2}-\mathrm{o}$ with respect to a film without photonic properties. Enhanced absorption was detected for all $x$ in $(1-x) 150-x 180-\mathrm{i}-\mathrm{nc}-\mathrm{TiO}_{2}-\mathrm{o}$, with a small red-shift in peak position from that of $150-\mathrm{i}-$ nc- $\mathrm{TiO}_{2}$-o. Although absorption peak at $\sim 360 \mathrm{~nm}$ persists throughout all compositions of $(1-x) 150-x 180$-i-nc- $\mathrm{TiO}_{2}-\mathrm{o}$, a decrease in absorption for $\lambda<325 \mathrm{~nm}$ is observed for $x=$ 0.57 and 0.7 . For these compositions, the increased light scattering may prevent light 
from penetrating the sample as the wavelength of light approaches close to the void size. On the other hand, changes in the absorbance spectra of (1-x)150-x210-i-nc- $\mathrm{TiO}_{2}-\mathrm{o}$ are much more significant than $(1-x) 150-x 180-\mathrm{i}-\mathrm{nc}-\mathrm{TiO}_{2}-\mathrm{o}$. With $210 \mathrm{~nm}$ as doping spheres, the peak position red-shifts with increasing $x$, from $350 \mathrm{~nm}$ (for $x=0$ ) to $365 \mathrm{~nm}$ (for $x=$ 1) and a dramatic decrease in the magnitude is observed. In addition, for compositions
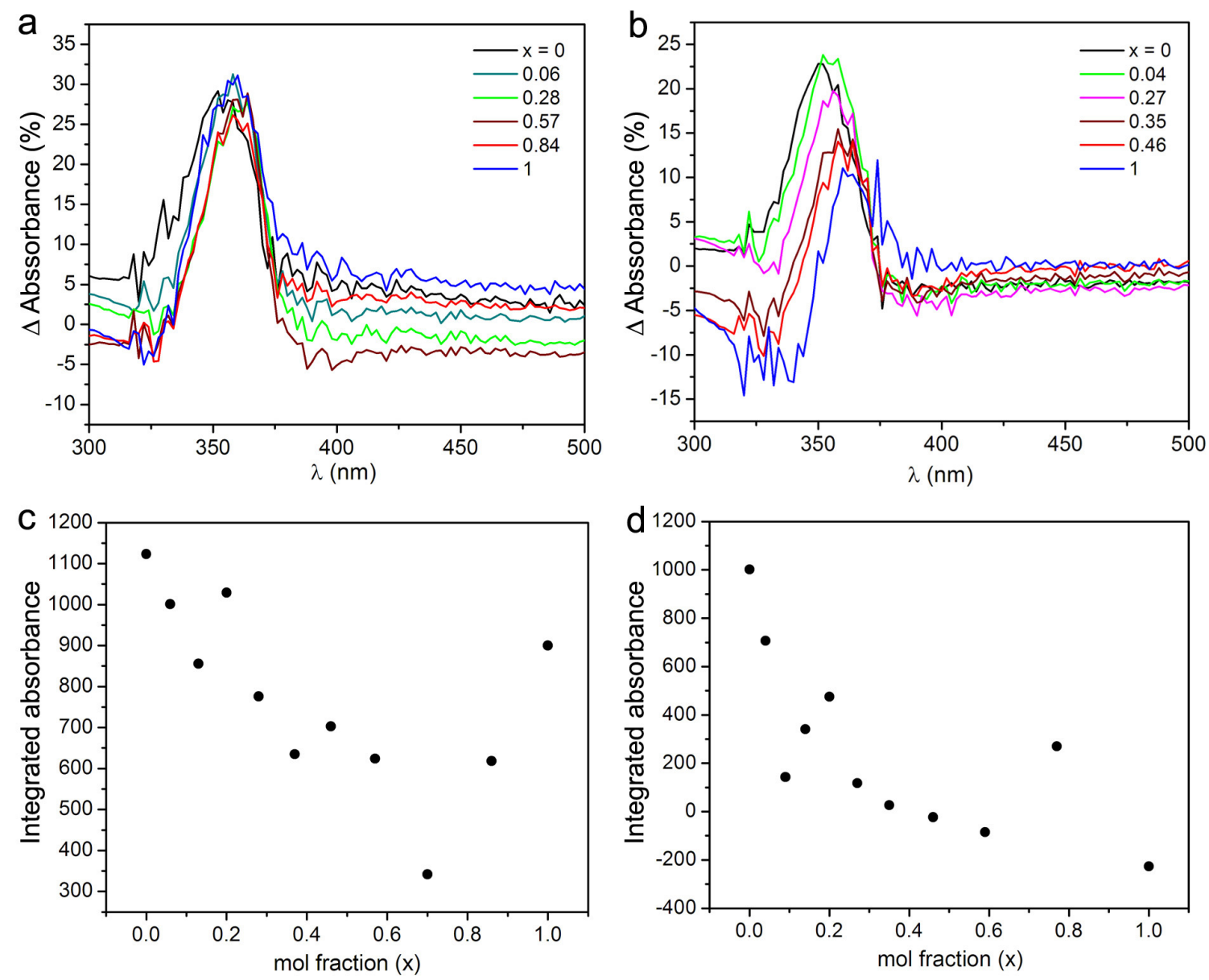

Figure 4.6. Changes in the absorbance spectra of $(1-x) 150-x 180-\mathrm{i}-\mathrm{nc}-\mathrm{TiO}_{2}-\mathrm{o}$ (a) and $(1-x) 150-$ $x 210$-i-nc- $\mathrm{TiO}_{2}-\mathrm{o}(\mathrm{b})$ after subtracting the absorbance of a reference $\mathrm{TiO}_{2}$ film. Total absorbance obtained from integration plotted as a function of $x$ for $(1-x) 150-x 180-\mathrm{i}-\mathrm{nc}^{-\mathrm{TiO}_{2}-\mathrm{o}}$ (c) and (1$x) 150-x 210-\mathrm{i}-\mathrm{nc}-\mathrm{TiO}_{2}-\mathrm{o}(\mathrm{d})$. 
with $x>0.35$ the absorbance of wavelengths shorter than $330 \mathrm{~nm}$ becomes lower than the reference film, suggesting that the disordered $(1-x) 150-x 210-\mathrm{i}-\mathrm{nc}-\mathrm{TiO}_{2}-\mathrm{O}$ absorb much less high energy UV light than $\mathrm{nc}^{-\mathrm{TiO}_{2}}$. In the case of $x=1$ (210-i-nc- $\left.\mathrm{TiO}_{2}-\mathrm{o}\right)$, the decrease in absorbance centered at $345 \mathrm{~nm}$ is the result of stop-band reflection. To obtain a clearer view of the changes in the total absorbance of the films, the areas under the absorbance curve were integrated and plotted as a function of $x$ (Fig. $4.6 \mathrm{c}$ and d). For (1$x$ )150-x 180-i-nc- $\mathrm{TiO}_{2}-\mathrm{o}$, total absorbance gradually decreases to about half of the original $x=0$ value before dipping further at $x=0.7$ and rising back up as composition nears $x=$

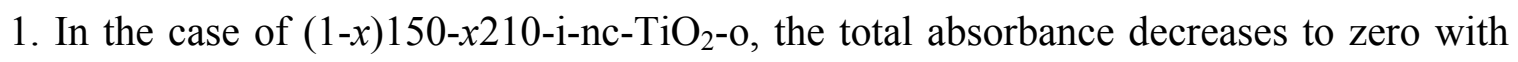
increasing $x$.

\subsubsection{Structure of Binary Inverse Titania Opals}

Figure 4.7 shows the SEM images of various compositions of (1-x)150-x180-i-nc$\mathrm{TiO}_{2}$-o. On visual inspection, all of the inverse structures show good short-range order; little deviation from the ideal closed-packing in the (111) plane is observed. The anatase nanocrystals $(\sim 10 \mathrm{~nm})$ forming the framework of the inverse structure are displayed in Fig. 4b, which also illustrates how the voids from $180 \mathrm{~nm}$ spheres are dispersed within those of $150 \mathrm{~nm}$. On the other hand, Fig. $4.7 \mathrm{c}$, where $x=0.37$, shows that the registry between the (111) lattice planes varies within an imaging area of $\sim 2 \mu \mathrm{m}^{2}$ as a result of the different sphere sizes. The observation of SEM images combined with optical spectra suggest that the short-range order is conserved in $(1-x) 150-x 180-\mathrm{i}-\mathrm{nc}^{-\mathrm{TiO}_{2}-\mathrm{o}}$ but the domain size of perfect fcc-packing of $150 \mathrm{~nm}$ spheres decreases with increasing $x$. The 
direction of [111] for most domains remains close to perpendicular to the glass substrate since Bragg diffraction is detected for all of the (1-x)150-x 180 templates.
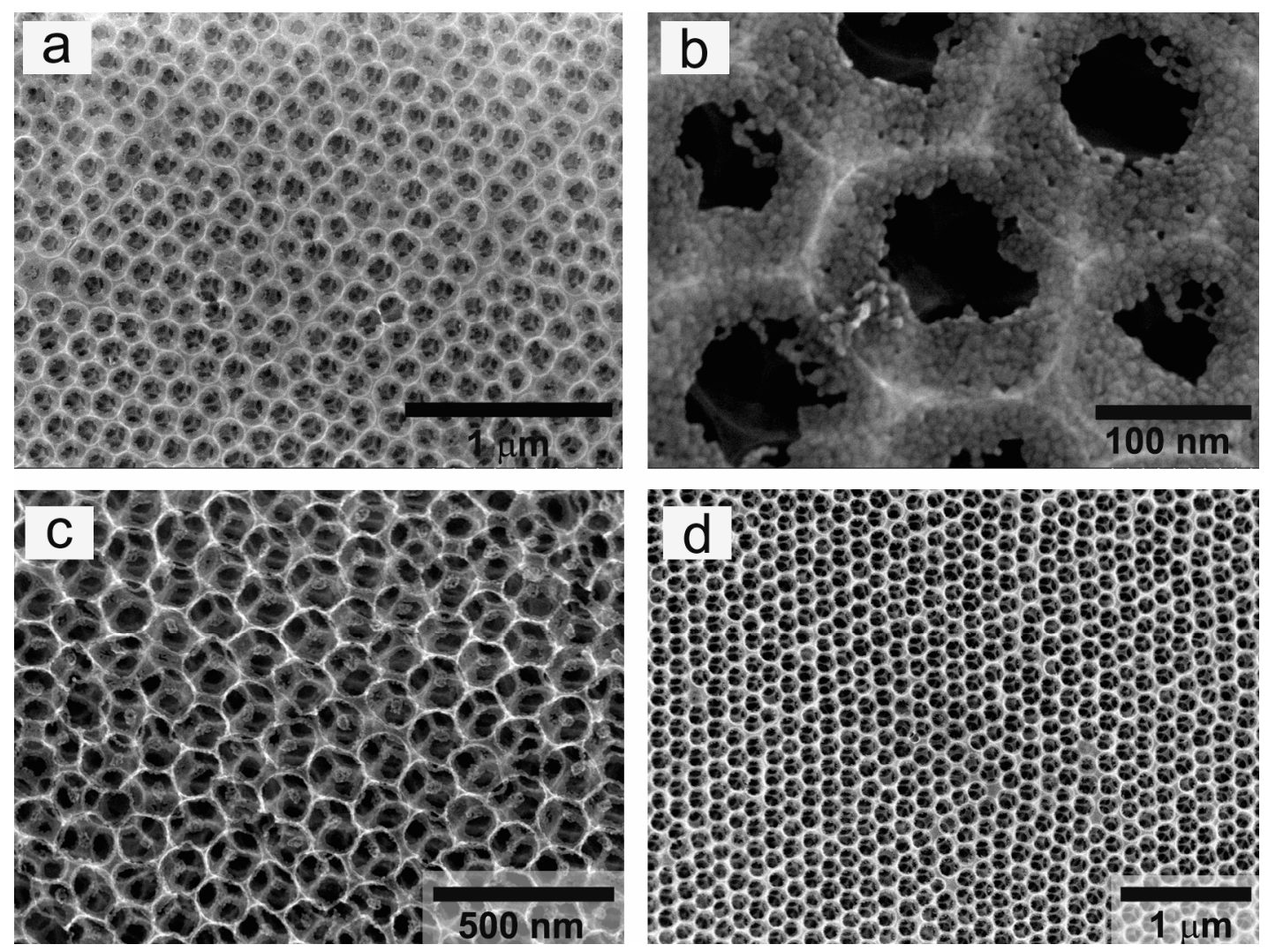

Figure 4.7. SEM images of $(1-x) 150-x 180-\mathrm{i}-\mathrm{nc}^{-\mathrm{TiO}_{2}-\mathrm{o}}$ with $x=0.13$ (a and b); 0.37 (c) and 0.57 (d).

The major difference in the structural disruption brought on by the $210 \mathrm{~nm}$ template spheres can be clearly seen in the SEM images in Fig. 4.8. At $x=0.10$, regions of ordered $150 \mathrm{~nm}$ voids interrupted by $210 \mathrm{~nm}$ voids can be observed (Fig. 4.8a). Unlike the $(1-x) 150-x 180$-i-nc- $\mathrm{TiO}_{2}$-o system where the void-packing maintains a hexagonal arrangement and the lattice appears relatively undisturbed, the packing of the $150 \mathrm{~nm}$ spherical voids in $(1-x) 150-x 210-\mathrm{i}-\mathrm{nc}-\mathrm{TiO}_{2}$-o rearranges upon even minor disruption by 
$210 \mathrm{~nm}$ voids. As a result, the closed-packing directions along [111] of the $150 \mathrm{~nm}$ domains vary to a greater extent, which also correlates well with the absence of stop-band diffraction of the (1-x)150-x210 template even at very small $x$ (Fig. 4.4c). Close examination of a series of SEM images of low $x$ suggests some phase segregation between $150 \mathrm{~nm}$ and $210 \mathrm{~nm}$ voids, also reported by Gates and Xia. ${ }^{[11]}$ The $210 \mathrm{~nm}$ voids are often found to be neighboring at least one other $210 \mathrm{~nm}$ void, as indicated by the arrows in Fig. 4.8a, while statistically it should not be a likely scenario at such low $x$. With increasing $x$, the packing of the spheres becomes very irregular and the packing efficiency drops greatly from $74 \%$ as can be noticed from the increased interstitial space
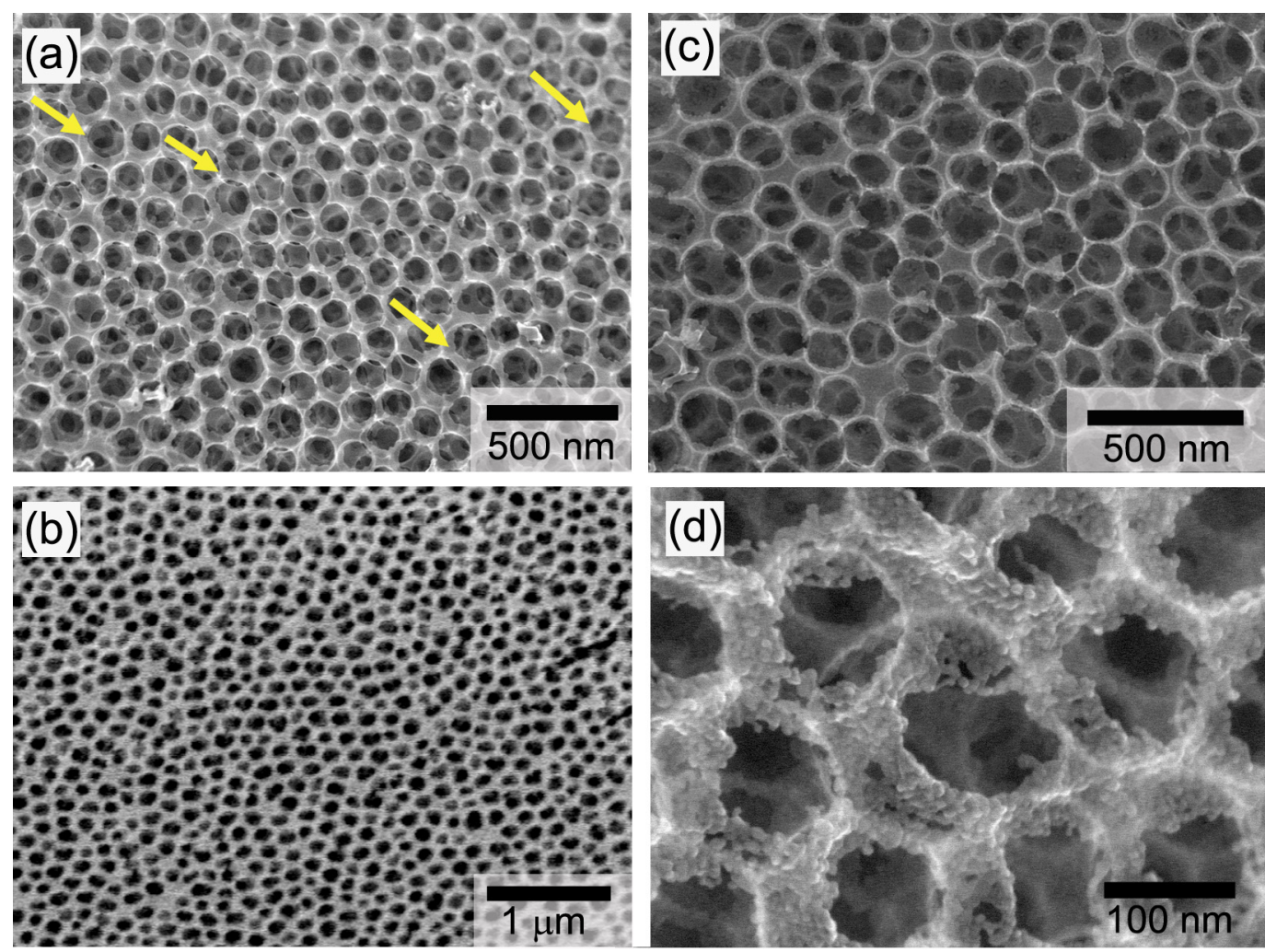

Figure 4.8. SEM images of $(1-x) 150-x 210-\mathrm{i}-\mathrm{nc}^{-} \mathrm{TiO}_{2}-\mathrm{o}$ with $x=0.09$ (a); 0.35 (b and c) and 0.77

(d). Some aggregation of the larger $210 \mathrm{~nm}$ voids is observed, as indicated by the arrows in (a). 
between the voids in Fig. $4.8 \mathrm{~b}(x=0.35)$. The packing efficiency of binary systems of different sphere sizes at fixed volume fraction has been theoretically studied by Kansal et al. ${ }^{[17]}$ According to their data, the composition for $x \sim 0.6$ in $(1-x) 150-x 180$ should have a packing efficiency of $65 \%$ if the spheres are randomly packed. However, the evaporativeself-assembly deposition method used in this work precludes such random packing and therefore the packing efficiency of $65 \%$ can only be regarded as a lower bound. Nonetheless one can expect the packing efficiency to decrease when binary compositions deviate from the end members as a result of more random packing of the spheres. Experimentally, the increase in void space of the template for compositions away from $x$ $=0$ or 1 was qualitatively observed when more titanium butoxide precursor was needed to completely infiltrate these templates.

\subsection{Photodegradation by Disordered Inverse Titania Opals}

\subsubsection{Binary Systems of (1-x)150-x180 and (1-x)150-x210}

The photodegradation efficiency of (1-x)150-x180-i-nc-TiO ${ }_{2}$-o under white light (>300 nm) was investigated employing the same method as before. Namely, the photodegradation of an adsorbed monolayer of methylene blue on $\mathrm{i}$-nc- $\mathrm{TiO}_{2}$-o was monitored by UV-Vis spectroscopy and the ratio of the decay constant of $\mathrm{i}-\mathrm{nc}-\mathrm{TiO}_{2}$-o to reference nanocrystalline $\mathrm{TiO}_{2}$, termed the enhancement factor $(\mathrm{EF})$, is calculated as a measure of the efficiency. The adsorption of monolayer dye molecules standardizes all samples with respect to their surface area. Figure 4.9 shows the EF as a function of $x$ for (1-x)150- $x 180$-i-nc- $\mathrm{TiO}_{2}$-o. For $x=0$, i.e. $150-\mathrm{i}-\mathrm{nc}-\mathrm{TiO}_{2}-\mathrm{o}$, an $\mathrm{EF}$ of 2.3 was achieved and attributed to slow photons of the photonic crystal while stop band reflection was 
effectively suppressed by anatase absorption. With increasing $x$, the overall enhancement decreases roughly by a factor of two from EF of 2.3 at $x=0$ to EF of $\sim 1.6$ at $x=0.13$ and then plateaus for $0.20<x<0.37$. The exponential-like decrease of EF from $x=0$ to 0.37 can be attributed to an increase of disorder in the system. The trend in EF follows closely with the decrease in domain size in Fig. 4.4 since the optical properties of the template should directly correlate with the photonic properties of the inverse structure. The domain size of fcc-packing of $150 \mathrm{~nm}$ remains above a critical threshold at $\sim 10$ layers, where multiple diffraction that gives rise to slow photons still dominates, also evidenced by the enhanced absorption in Fig. 4.6a. Hence the EF remains at 1.5 for $x$ up to 0.37. A further increase in the concentration of large spheres leads to a final drop of the EF at $0.46<x<$ 0.57. This composition range may represent the breakdown point at which the clusters of $150 \mathrm{~nm}$ spherical voids become smaller than the critical size. Photonic effects attributed to domains formed by $150 \mathrm{~nm}$ voids consequently vanish.

Looking at compositions near the other end member, i.e. 180 -i-nc- $\mathrm{TiO}_{2}-\mathrm{o}$, the $\mathrm{EF}$ decreases with decreasing $x$ from $x=1$ which correlates with the increasing amount of disorder as $150 \mathrm{~nm}$ spheres are incorporated into $180 \mathrm{~nm}$ lattice. The substitution of 150 $\mathrm{nm}$ spheres into $180 \mathrm{~nm}$ lattice increases the filling fraction of $\mathrm{TiO}_{2}$ in the inverse structure, as evidenced in the red-shift of the stop-band for $x=0.84$ and 0.70 in comparison to $x=1$ in Fig. 4.5a. This increase in the volume fraction of $\mathrm{TiO}_{2}$ leads to an increase in the number of defects in the high dielectric part of the photonic crystal, hence disturbing the modes which are utilized for the photodegradation. The current observation, where (1-x)150-x180-i-nc- $\mathrm{TiO}_{2}$-o with $x=0.84$ is less efficient than $x=1$, is also consistent with the finding in Chapter 3. Namely, the EF decreases when stop-band 
(in air) red-shifts in the wavelength range of $300-370 \mathrm{~nm}$. The overall dependence of EF on the composition in the binary system of $(1-x) 150-x 180-\mathrm{i}-\mathrm{nc}-\mathrm{TiO}_{2}-\mathrm{o}$ shows that substantial amount of disorder can be tolerated while maintaining respectable $50 \%$ photodegradation enhancement.

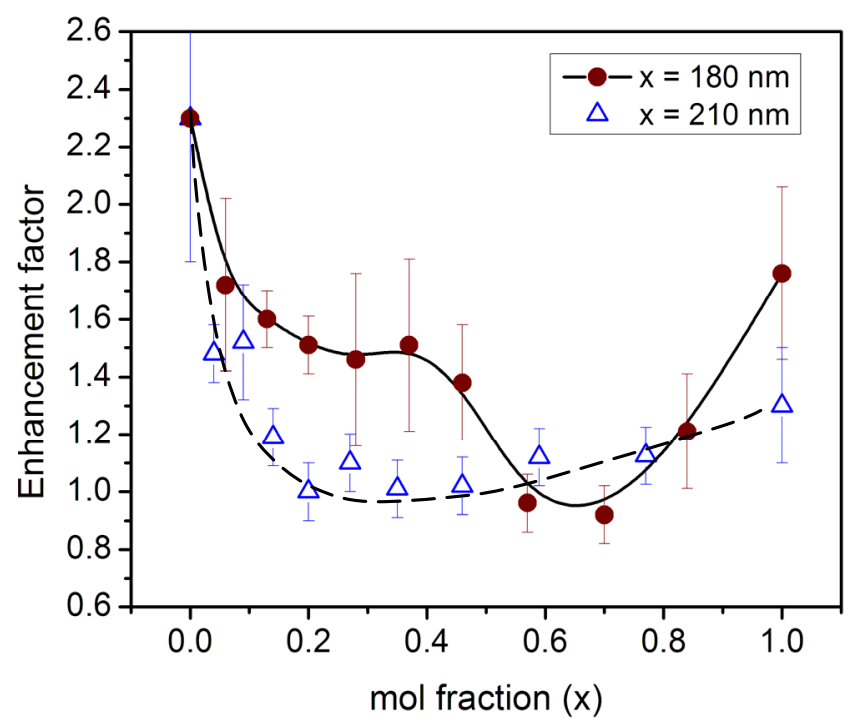

Figure 4.9. Enhancement factor of the photocatalytic efficiency of $(1-x) 150-x 180-\mathrm{i}-\mathrm{nc}-\mathrm{TiO}_{2}-\mathrm{O}$ and (1-x)150-x210-i-nc- $\mathrm{TiO}_{2}-\mathrm{o}$ as a function of composition. The solid and dashed lines are guides to the eye.

The effect of disorder on the photodegradation efficiency is further explored with the binary system of $(1-x) 150-x 210-\mathrm{i}-\mathrm{nc}-\mathrm{TiO}_{2}-\mathrm{o}$. As the optical spectra and SEM images suggest, the larger $210 \mathrm{~nm}$ spheres disrupt the lattice of $150 \mathrm{~nm}$ spheres much more drastically than $180 \mathrm{~nm}$ spheres. Therefore one would anticipate the domain size of 150 $\mathrm{nm}$ spheres to decrease at a faster rate in $(1-x) 150-x 210-\mathrm{i}-\mathrm{nc}-\mathrm{TiO}_{2}$-o compared to (1x) 150- $x 180$-i-nc- $\mathrm{TiO}_{2}$-o. Figure 4.9 in fact shows a much faster decrease in $\mathrm{EF}$ without 
any plateau region for the binary system $(1-x) 150-x 210-\mathrm{i}-\mathrm{nc}-\mathrm{TiO}_{2}-\mathrm{o}$. At $x=0.1$, small domains of $150 \mathrm{~nm}$ voids can still be observed (Fig. 4.8a) and some enhancement is observed. However, no short-range order of $150 \mathrm{~nm}$ or $210 \mathrm{~nm}$ voids can be seen for middle compositions, and the EF effectively decreases to $\sim$. Interestingly, the trend in EF closely resembles the trend in the total absorbance for the two binary systems (Fig. 4.6c and d). As the photodegradation efficiency not only depends on the amount of absorption but also quantum efficiency, which has been shown to be spectraldependent, ${ }^{[18]}$ the correlation can be looked upon as qualitative but not quantitative. For example, although there exist some absorption enhancements at $350-370 \mathrm{~nm}$ in (1$x) 150-x 210$-i-nc- $\mathrm{TiO}_{2}$-o for $0.1<x<0.3$, the overall photodegradation efficiency for these compositions was not higher than $\mathrm{EF} \sim 1$. This difference may arise from the lower quantum yield of low energy UV light. The correlation between the EF and the total absorbance for different compositions attests that structural disorder affects the slowphoton enhanced absorption, which in turn relates directly to the photodegradation efficiency of $\mathrm{TiO}_{2}$.

Since the opal templates were prepared from solutions containing a fixed total volume of the spheres, increasing $x$ corresponds to a decrease in the volume fraction of $150 \mathrm{~nm}$ spheres. Consequently the enhancement may be proposed to relate to the density of $150 \mathrm{~nm}$ voids rather than the packing order. To test this hypothesis, a thicker film of (1-x) $150-x 210-\mathrm{i}-\mathrm{nc}-\mathrm{TiO}_{2}$-o with $x=0.14$ and volume fraction of $150 \mathrm{~nm}$ spheres the same as $x=0$ was prepared. The EF for the thick film was 1.14 , within the experimental error of the thinner film. Therefore the density argument can be dismissed, and one can conclude that structural order is the main factor responsible for the enhancement, which 
is also supported by the observation that EF decays at a faster rate with increasing $x$ in (1x)150-x210-i-nc- $\mathrm{TiO}_{2}$-o than in $(1-x) 150-x 180-\mathrm{i}-\mathrm{nc}-\mathrm{TiO}_{2}$-o.

\subsubsection{Effect of Light Scattering}

Past research has shown that structural disorder can increase the amount of light scattering within the photonic crystal. Subsequently, light scattering and the corresponding longer path length of light in the material have been explored to enhance the efficiency of dye-sensitized $\mathrm{TiO}_{2}$ solar cells. ${ }^{[19-21]}$ In $(1-x) 150-x 180-\mathrm{i}-\mathrm{nc}-\mathrm{TiO}_{2}-\mathrm{o}$ and (1-x)150-x210-i-nc- $\mathrm{TiO}_{2}-\mathrm{o}$, the samples become visibly more opaque with increasing disorder. However, none of the disordered samples show a higher enhancement than the end members ( $x=0$ or 1$)$, suggesting that forward scattering at the wavelengths near the $\mathrm{TiO}_{2}$ absorption has limited contribution to the enhancement. Furthermore if forward scattering at the disordered region was a key factor, then $(1-x) 150-x 210-\mathrm{i}-\mathrm{nc}-\mathrm{TiO}_{2}-\mathrm{O}$ should have a higher EF than $(1-x) 150-x 180-\mathrm{i}-\mathrm{nc}-\mathrm{TiO}_{2}$-o for a given $x$ as $(1-x) 150-x 210-\mathrm{i}$ nc- $\mathrm{TiO}_{2}$-o has a much higher degree of disorder. Conversely, it is possible that scattering from disordered regions decreases the amount of light penetrating through the material, thereby decreasing the amount of $\mathrm{TiO}_{2}$ effectively interacting with light. Unfortunately, the interplay of photonic effect and light scattering could not be deconvoluted in the binary systems containing $150 \mathrm{~nm}$ voids to clearly distinguish their contributions to the overall enhancement. To overcome this problem, a study of scattering samples in the absence of any photonic properties is needed. In Chapter 3, it has demonstrated that inverse opal made from $130 \mathrm{~nm}$ template sphere did not exhibit any photonic effect since the strong absorption of $\mathrm{TiO}_{2}$ effectively eliminated the existence of both stop-band 
(extrapolated to be at $280 \mathrm{~nm}$ ) and slow photons. A disordered inverse structure containing mostly $130 \mathrm{~nm}$ voids thus should serve as a good system to investigate the role of scattering from disordered regions since no photonic effect is present. The EF of disordered $130-180-\mathrm{i}-\mathrm{nc}-\mathrm{TiO}_{2}-\mathrm{o}(x=0.1)$ is comparable to the inverse opal of wellordered $130 \mathrm{~nm}$ voids (EF of 1.05 vs. 1.10). This observation combined with the trend of EF for the (1-x)150-x $180-\mathrm{i}-\mathrm{nc}-\mathrm{TiO}_{2}-\mathrm{o}$ and $(1-x) 150-x 210-\mathrm{i}-\mathrm{nc}-\mathrm{TiO}_{2}$-o strongly support the idea that short-range structural order is the dominant factor in the photocatalytic enhancement. Additionally, the photodegradation activity of $180-240-\mathrm{i}-\mathrm{nc}-\mathrm{TiO}_{2}-\mathrm{o}(x=$ 0.1) was examined, and it did not show any enhancement either when compared to the reference. The EF for i-nc- $\mathrm{TiO}_{2}$-o with different combination of sphere sizes obtained from irradiation at normal incidence and 45-degrees off-normal are shown in Fig. 4.10.

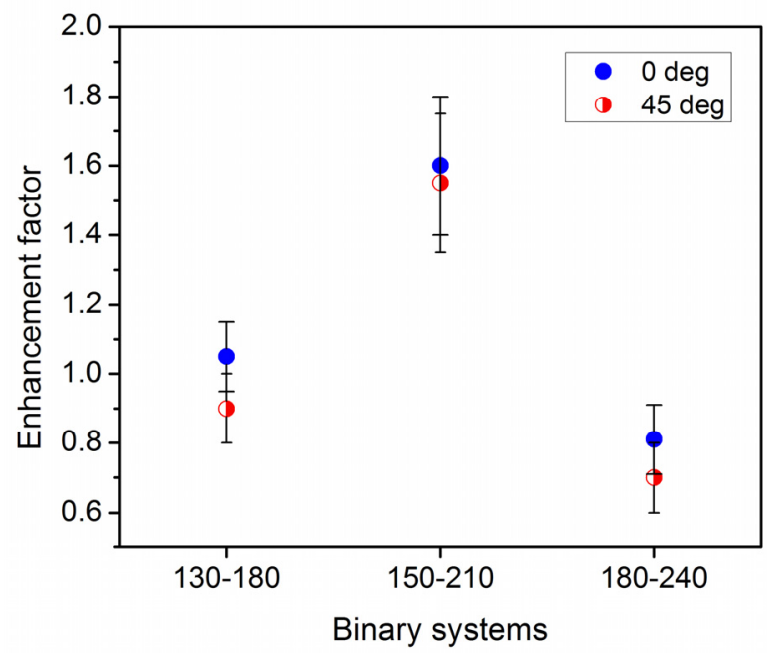

Figure 4.10. Enhancement factor for inverse structures of various binary systems with different sphere size combination $(x=0.1)$ obtained at irradiation angles normal and 45-degrees off-normal of the films. 
The photodegradation efficiency of these samples is found to be angle-independent due to the absence of photonic properties or the random orientation of small $150 \mathrm{~nm}$ domains in the case of $150-210-\mathrm{i}-\mathrm{nc}-\mathrm{TiO}_{2}-\mathrm{o}$, contrary to the angle-dependent behavior of the ordered inverse opals. Unlike other studies which suggest multiple scattering being favorable for increasing the path length of light, the present study indicates ordered structures to be the most advantageous architecture for enhancing the photodegradation efficiency of $\mathrm{TiO}_{2}$.

\subsection{Conclusions}

Based on the systematic studies of the two binary systems (1-x)150-x180-i-nc$\mathrm{TiO}_{2}-\mathrm{o}$ and $(1-x) 150-x 210-\mathrm{i}-\mathrm{nc}-\mathrm{TiO}_{2}-\mathrm{o}$, the photodegradation efficiency of anatase titania is governed by the domain size of close-packed $150 \mathrm{~nm}$ spherical voids, which determine the amount of optical enhancement. The domain size remains sufficiently large for slow photons to prevail to give $\sim 50 \%$ enhancement even when fractions as high as 0.4 of 150 $\mathrm{nm}$ voids were substituted by $180 \mathrm{~nm}$. Increasing the disorder decreased the photodegradation efficiency and thus no advantage of increasing the amount of light scattering was found. The findings demonstrate firsthand the effect of disorder on photonic properties and how structural imperfection can impact the practical application of $\mathrm{TiO}_{2}$-based photonic crystals. While highly monodisperse template spheres are desired, they are not absolutely necessary for practical environmental remediation applications using $\mathrm{TiO}_{2}$ inverse opals. The high tolerance to structural disorder opens the door for the realistic use of colloidal photonic crystals that may be assembled from spheres of limited-monodispersity or fabricated by means that are more versatile and less time-consuming such as air-spraying or spin-coating. 


\subsection{References}

[1] J. D. Joannopoulos, P. R. Villeneuve, S. Fan, Nature 1997, 386, 143.

[2] L. I. Halaoui, N. M. Abrams, T. E. Mallouk, J. Phys. Chem. B 2005, 109, 6334.

[3] S. Nishimura, N. Abrams, B. A. Lewis, L. I. Halaoui, T. E. Mallouk, K. D. Benkstein, J. Van de Lagemaat, A. J. Frank, J. Am. Chem. Soc. 2003, 125, 6306.

[4] A. Mihi, H. Miguez, J. Phys. Chem. B 2005, 109, 15968.

[5] Z. Y. Li, Z. Q. Zhang, Phys. Rev. B 2000, 62, 1516.

[6] R. Rengarajan, D. Mittleman, C. Rich, V. Colvin, Phys. Rev. E 2005, 71.

[7] E. Vekris, V. Kitaev, D. D. Perovic, J. S. Aitchison, G. A. Ozin, Adv. Mater. 2008, $20,1110$.

[8] D. M. Beggs, M. A. Kaliteevski, R. A. Abram, D. Cassagne, J. P. Albert, J. Phys.: Condens. Matter 2005, 17, 1781.

[9] M. A. Kaliteevski, J. Manzanares Martinez, D. Cassagne, J. P. Albert, Phys. Rev. B 2002, 66, 1131011.

[10] C. Conti, A. Fratalocchi, Nature Phys. 2008, 4, 794

[11] B. Gates, Y. Xia, Appl. Phys. Lett. 2001, 78, 3178.

[12] C. Paquet, M. Allard, G. Gledel, E. Kumacheva, J. Phys. Chem. B 2006, 110, 1605.

[13] E. Palacios-Lidon, B. H. Juarez, E. Castillo-Martinez, C. Lopez, J. Appl. Phys. 2005, 97, 063502 .

[14] P. Jiang, J. F. Bertone, K. S. Hwang, V. L. Colvin, Chem. Mater. 1999, 11, 2132.

[15] E. Yablonovitch, T. J. Gmitter, R. D. Meade, A. M. Rappe, K. D. Brommer, J. D. Joannopoulos, Phys. Rev. Lett. 1991, 67, 3380. 
http://dx.doi.org/10.1103/PhysRevLett.67.3380

[16] R. J. Spry, D. J. Kosan, Appl. Spectrosc. 1986, 40, 782.

[17] A. R. Kansal, S. Torquato, F. H. Stillinger, J. Chem. Phys. 2002, 117, 8212.

[18] A. Emeline, A. Salinaro, N. Serpone, J. Phys. Chem. B 2000, 104, 11202.

[19] A. Usami, Sol. Energy Mater. 1999, 59, 163.

[20] G. Rothenberger, P. Comte, M. Gratzel, Sol. Energy Mater. 1999, 58, 321.

[21] S. Hore, P. Nitz, C. Vetter, C. Prahl, M. Niggemann, R. Kern, Chem. Comm. 2005, 2011. 


\title{
CHAPTER 5
}

\section{Heterogeneous Photocatalysis with Inverse Titania Opals}

(Reproduced in part with permission from J. Mater. Chem. 2009, DOI:

10.1039/B900965E. Copyright 2009 Royal Society of Chemistry)

\begin{abstract}
The first example of slow-photon amplified photochemical reaction using inverse $\mathrm{TiO}_{2}$ opals and the sensitivity of such optical amplification to structural disorder were demonstrated in the previous two chapters. However thus far a solid-state photodegradation experiment was implemented in order to elucidate unambiguously the photonic effect; it eliminated all other factors that may influence the rate of photodegradation. Putting the fundamental scientific inquiry aside, much of the interest of $\mathrm{TiO}_{2}$ lies in environmental remediation where applications like water treatment and air purification are actively pursued. Therefore it is of practical importance to investigate how the $\mathrm{i}-\mathrm{nc}-\mathrm{TiO}_{2}$-o performs in a heterogeneous environment. In a solid-liquid system, there are other factors that influence the rate of photodegradation, such as the rate of diffusion, the surface area of the photocatalyst, and the interaction of molecules in solution with the solid support. In terms of changes in the photonic properties, infiltration of air voids of any photonic structure with other material will effectively decrease the dielectric contrast. Hence a study of the effect of individual factors on the overall efficiency of solution photocatalysis by $\mathrm{i}-\mathrm{nc}-\mathrm{TiO}_{2}-\mathrm{O}$ was carried out and presented herein.
\end{abstract}




\subsection{Introduction}

Heterogeneous photocatalysis entails many different factors that may influence the overall performance of the photocatalyst. With the pollutants in the solution phase and $\mathrm{TiO}_{2}$ as powder or supported-film, the photodegradation proceeds via several steps. ${ }^{[1]}$ First the diffusion of molecules to $\mathrm{TiO}_{2}$ has to occur followed by some surface adsorption or surface interaction that would allow the photogenerated electron-hole (or the $\mathrm{O}_{2}{ }^{-\bullet}$ and $\mathrm{OH}^{\bullet}$ radicals they produce) to react and transform the molecule. Then, the products or intermediates need to diffuse away from the surface of $\mathrm{TiO}_{2}$ so that photocatalytic active sites are regenerated. As a result, not only is the photoactivity of $\mathrm{TiO}_{2}$ important, the diffusion of ions and molecules and surface interactions in a heterogeneous environment also play pivotal roles. The rate of diffusion is closely related to the porosity of the photocatalyst, which in turn correlates with the surface area that is available for reaction. Increasing the surface area by making the $\mathrm{TiO}_{2}$ meso- or macro-porous in fact has lead to a superior photocatalytic activity than dense $\mathrm{TiO}_{2}$ film. ${ }^{[2-6]}$ Detailed study on the pore geometry further suggests that the architecture and connectivity of the pores can influence the rate of photoreaction. ${ }^{[7]}$ On the other hand, the properties of the solution, such as the presence of other metal ions ${ }^{[8]}, \mathrm{pH}^{[9]}$ and degree of aeration ${ }^{[10]}$, affect the interaction of molecules with $\mathrm{TiO}_{2}$ at the solid-liquid interface. In order to probe the slow-photon enhancement of $\mathrm{i}$-nc- $\mathrm{TiO}_{2}-\mathrm{o}$ in solution photocatalysis, the aforementioned factors need to be accounted for. As photonic properties arise from the dielectric contrast in a periodic structure, the presence of water in the voids of $\mathrm{i}$-nc- $\mathrm{TiO}_{2}-\mathrm{o}$ reduces this refractive index contrast and effectively reduces the slow-photon group velocity and concomitant enhancement according to theory. In this chapter, the solution photocatalysis 
using i-nc- $\mathrm{TiO}_{2}-\mathrm{O}$ is investigated with the aim at isolating photonic crystal from diffusion and surface area effects. In addition, a method to overcome the low refractive-index contrast problem is presented.

\subsection{Experimental Methods}

\subsubsection{Increasing the Filling Fraction of $\mathrm{TiO}_{2}$}

Calcined i-nc- $\mathrm{TiO}_{2}-\mathrm{o}$ was obtained using methods outlined in Chapter 3. The i-nc$\mathrm{TiO}_{2}$-o films were immersed in a solution containing $0.015 \mathrm{M}\left(\mathrm{NH}_{4}\right)_{2} \mathrm{TiF}_{6}, 0.011 \mathrm{M} \mathrm{H}_{3} \mathrm{BO}_{3}$ and $0.01 \mathrm{M} \mathrm{HCl}$ at $65{ }^{\circ} \mathrm{C}$ (all chemicals were from Aldrich). After half an hour (or the specified time), the films were removed from the solution, rinsed with water and dried. The as-deposited $\mathrm{TiO}_{2}$ was amorphous therefore a second calcination step to $450{ }^{\circ} \mathrm{C}$ for 4 hours was carried out to crystallize the additional $\mathrm{TiO}_{2}$ to the anatase phase.

\subsubsection{Determination of the Mass of Films}

The mass of the films were measured using a microbalance (ATI/CAHN C-34 model) with $1.0 \mu$ g precision. The mass of the film on a glass substrate was measured first, followed by scraping off the film and reweighing the glass substrate. The difference corresponds to the mass of film of $\sim 1 \times 2 \mathrm{~cm}^{2}$ in dimension. The reported values were obtained from an average over two to three films with error from the standard deviation.

\subsubsection{Specific Surface Area from Dye Adsorption-Desorption}

For dye adsorption, each film was placed in $10-\mathrm{mL}$ solution of $7 \mu \mathrm{M}$ methylene blue at $\mathrm{pH} 8$ in the dark. The absorbance of the films was measured and compared with 
monolayer coverage data (Chapter 3) for reference. To determine the absolute number of molecules of dye adsorbed, each film was immersed into $5 \mathrm{~mL}$ acidic water at $\mathrm{pH} 3$ for 1 hour (in the absence of light) to desorb methylene blue. The absorbance of the solutions was then measured using a UV-Vis spectrophotometer and the concentration extrapolated from a calibration curve. Typical desorbed dye concentrations were in the range of 0.9 $1 \mu \mathrm{M}$ where linear interpolation according to Beer-Lambert's law actually does not apply. So an exponential-like calibration curve was mapped out in this low concentration region for the determination of desorbed dye concentration. The reported values were obtained from an average over three runs with error from the standard deviation.

\subsubsection{Solution Photocatalysis}

White-light photodegradation of $7 \mu \mathrm{M}$ methylene blue in water at pH 8 by i-nc$\mathrm{TiO}_{2}-\mathrm{O}$ was carried out using a Xe lamp with a filter that cuts off $\lambda<300 \mathrm{~nm}$. The solution was in a large homemade rectangular cell $\left(2 \times 2 \times 7 \mathrm{~cm}^{3}\right)$ with the surface of the film and the front wall of the cuvette aligned perpendicular to the light beam. The solution was continuously stirred and samples were taken every 3 minutes and the absorbance measured.

\subsection{Effect of Filling Fraction on the Group Velocity}

Initial investigation of aqueous photocatalysis with $\mathrm{i}-\mathrm{nc}-\mathrm{TiO}_{2}-\mathrm{o}$ suggested insignificant enhancement from photonic effects. On closer examination with SWA modeling, it was revealed that the group velocity depends on the dielectric contrast between material of the inverse framework and that of the spherical voids. Figure 5.1 
shows the theoretical EF of $\mathrm{i}$-nc- $\mathrm{TiO}_{2}-\mathrm{O}$ in water with different filling fraction of $\mathrm{TiO}_{2}$ that occupies the $26 \%$ framework volume of an inverse fcc structure. Shown in Chapter 3 , the calcined i-nc- $\mathrm{TiO}_{2}-\mathrm{O}$ consists of nanocrystals with $\sim 30 \mathrm{vol} \%$ of $\mathrm{TiO}_{2}$ in the framework. The rest of the i-nc- $\mathrm{TiO}_{2}-\mathrm{o}(70 \%$ of $26 \%$ framework and $100 \%$ of $74 \%$ of spherical voids) is the medium that the inverse opal is placed in. In water, the EF is calculated to be just 1.3 at the wavelengths of the slow photons for the as-prepared i-nc$\mathrm{TiO}_{2}-\mathrm{O}$, compared to $\mathrm{EF}$ of 2 in air. Increasing the filling fraction of $\mathrm{TiO}_{2}$, however, can boost the enhancement to almost twofold if dense $\mathrm{TiO}_{2}(70 \%)$ made up the framework. Hence the following sections embark on the goal of increasing the filling fraction of $\mathrm{TiO}_{2}$ in $\mathrm{i}$-nc- $\mathrm{TiO}_{2}-\mathrm{O}$ in order to increase the slow-photon enhancement in an aqueous environment.

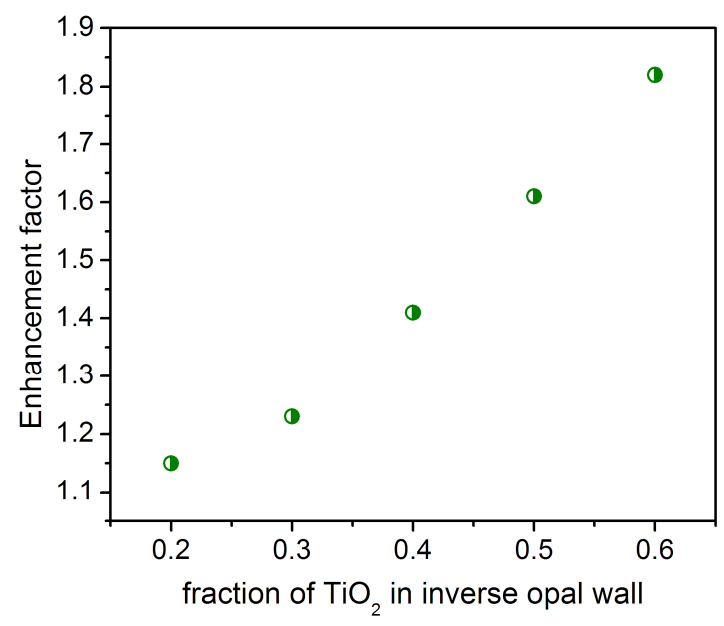

Figure 5.1. Theoretical enhancement factor of $\mathrm{i}-\mathrm{nc}-\mathrm{TiO}_{2}-\mathrm{o}$ in water as a function of the volume fraction of $\mathrm{TiO}_{2}$ in the inverse opal framework, as calculated by SWA.

\subsection{Characterization of Inverse $\mathrm{TiO}_{2}$ Opals with Higher Filling Fraction}

To increase the wall thickness of i-nc- $\mathrm{TiO}_{2}-\mathrm{o}$, additional $\mathrm{TiO}_{2}$ was deposited using the $\mathrm{TiF}_{6}{ }^{2-}$ complex, also known as Liquid Phase Deposition. ${ }^{[11]}$ The ligand- 
exchange equilibrium of the $\mathrm{TiF}_{6}{ }^{2-}$ complex (eqn. 5.1) can be shifted by adding a $\mathrm{F}^{-}$ scavenger such as $\mathrm{H}_{3} \mathrm{BO}_{3}$ to yield hydroxylated $\mathrm{TiO}_{2}$, as shown below. ${ }^{[12,13]}$

$$
\begin{aligned}
& {\left[\mathrm{TiF}_{6}\right]^{2-}+n \mathrm{H}_{2} \mathrm{O} \rightleftharpoons\left[\mathrm{TiF}_{6-n}(\mathrm{OH})_{n}\right]^{2-}+n \mathrm{HF}} \\
& \mathrm{H}_{3} \mathrm{BO}_{3}+4 \mathrm{HF} \rightleftharpoons \mathrm{BF}_{4}^{-}+\mathrm{H}_{3} \mathrm{O}^{+}+2 \mathrm{H}_{2} \mathrm{O}
\end{aligned}
$$

The formation of $\mathrm{TiO}_{2}$ can be finely tuned by controlling the concentration of reactants and duration and temperature of the reaction. The main advantage of this method is the lack of shrinkage during deposition that is most often associated with sol-gel precursors. The direct infiltration of polystyrene opal template using $\mathrm{TiF}_{6}{ }^{2-}$, however, did not yield high quality inverse opal due to a surface overlayer and some delamination of the film from the glass substrate. Instead, $\mathrm{TiF}_{6}{ }^{2-}$ treatment was performed on calcined i-nc- $\mathrm{TiO}_{2}-\mathrm{o}$. Figure 5.2 shows the SEM images of $\mathrm{i}-\mathrm{nc}-\mathrm{TiO}_{2}$-o treated with $\mathrm{TiF}_{6}{ }^{2-}$ for different lengths of time. From the thin walls of the original i-nc- $\mathrm{TiO}_{2}-\mathrm{o}(\mathrm{a})$, the walls thicken as reaction time increases to 30 minutes (b) and 45 minutes (c). The structural integrity is well preserved as can be seen in Fig. 5.2d. Optimal reaction time is determined to be 30 minutes, as the voids start to close with prolonged deposition.

The evolution of the photonic stop band with increasing $\mathrm{TiO}_{2}$ filling fraction is shown in Fig. 5.3. For template sphere size of $130 \mathrm{~nm}$, the original i-nc- $\mathrm{TiO}_{2}$-o does not show Bragg reflection in water due to the small size of the sphere and the low filling fraction of $\mathrm{TiO}_{2}$; the estimated stop-band position of as-prepared 130-nm i-nc- $\mathrm{TiO}_{2}$-o in water is $300 \mathrm{~nm}$, a region where reflection is completely suppressed by the strong $\mathrm{TiO}_{2}$ absorption. By increasing the thickness of the $\mathrm{TiO}_{2}$ wall, the photonic stop band redshifts such that a small reflection peak at $330 \mathrm{~nm}$ emerges and grows in intensity over the reaction time. The higher filling fraction of $\mathrm{TiO}_{2}$ in $\mathrm{i}-\mathrm{nc}-\mathrm{TiO}_{2}-\mathrm{O}$ in theory lowers the 

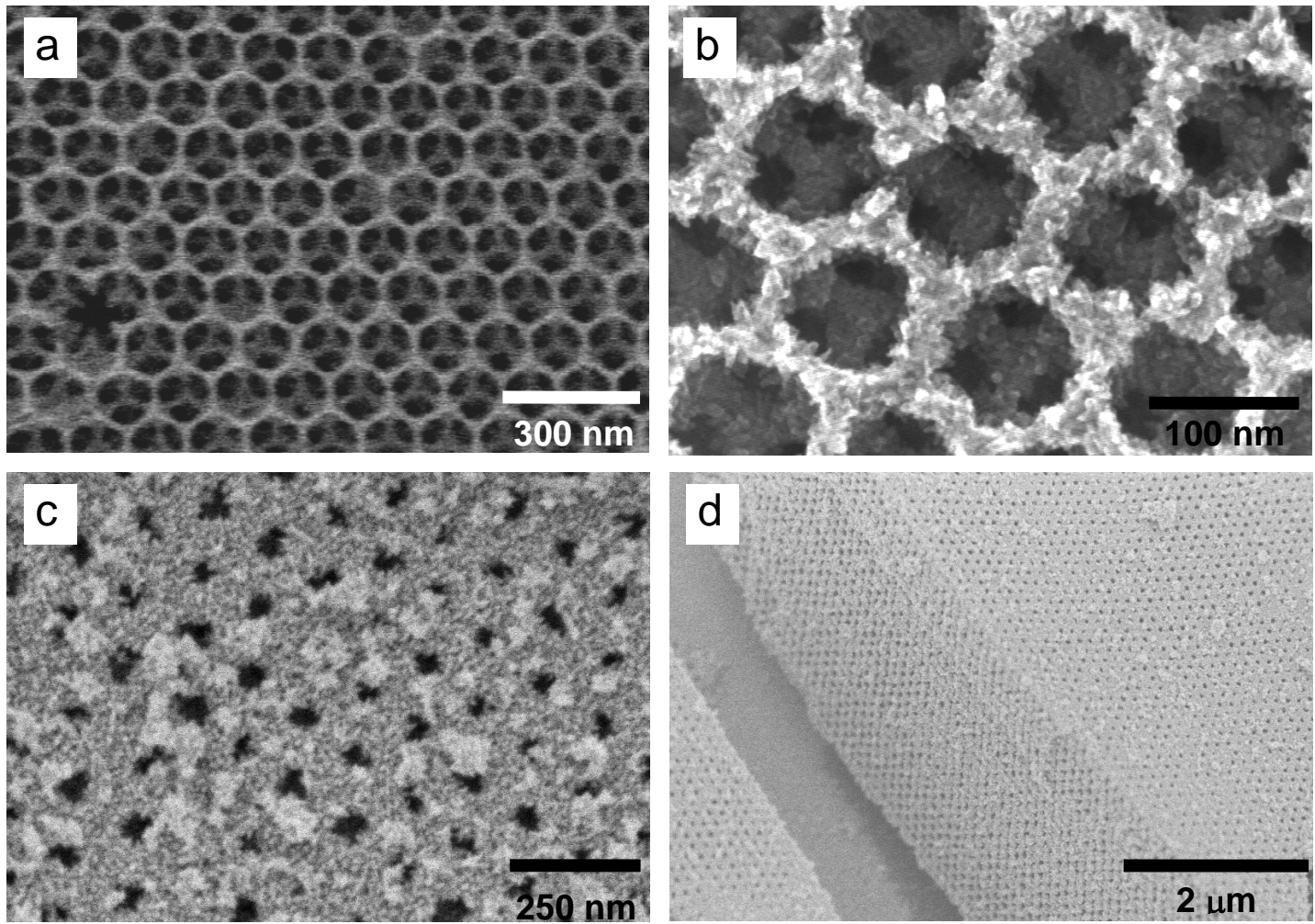

Figure 5.2. SEM images of $\mathrm{i}$-nc- $\mathrm{TiO}_{2}-\mathrm{O}$ as-prepared (a) and after 30 minutes (b) and 45 minutes (c, d) of $\mathrm{TiF}_{6}{ }^{2-}$ treatment. The open structure of the film is maintained.

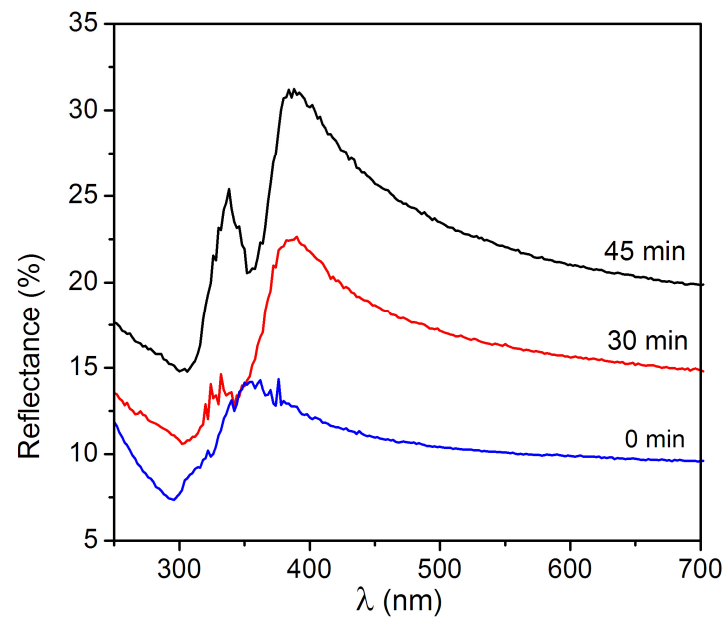

Figure 5.3. Reflectance spectra of $130-\mathrm{nm}$ templated i-nc- $\mathrm{TiO}_{2}-\mathrm{o}$ measured in water upon increasing the filling fraction of $\mathrm{TiO}_{2}$. 
group velocity and should therefore increase the path length of light and further enhance $\mathrm{TiO}_{2}$ absorption.

Figure 5.4 shows the reflectance spectra of $\mathrm{i}-\mathrm{nc}-\mathrm{TiO}_{2}-\mathrm{O}$ of different sphere sizes after treatment with $\mathrm{TiF}_{6}{ }^{2-}$ for 30 minutes. The stop bands are red-shifted by $10-20 \mathrm{~nm}$ as compared to their original positions. Since the refractive index of $\mathrm{TiO}_{2}$ is highly dispersive near its absorption edge, we used SWA to model the changes in the volume fraction of $\mathrm{TiO}_{2}$ as a result of the $\mathrm{TiF}_{6}{ }^{2-}$ treatment. We estimate that the vol\% of $\mathrm{TiO}_{2}$ increased to 45 - $50 \%$ from the original $30 \%$ in the as-prepared $\mathrm{i}-\mathrm{nc}-\mathrm{TiO}_{2}-\mathrm{o}$.

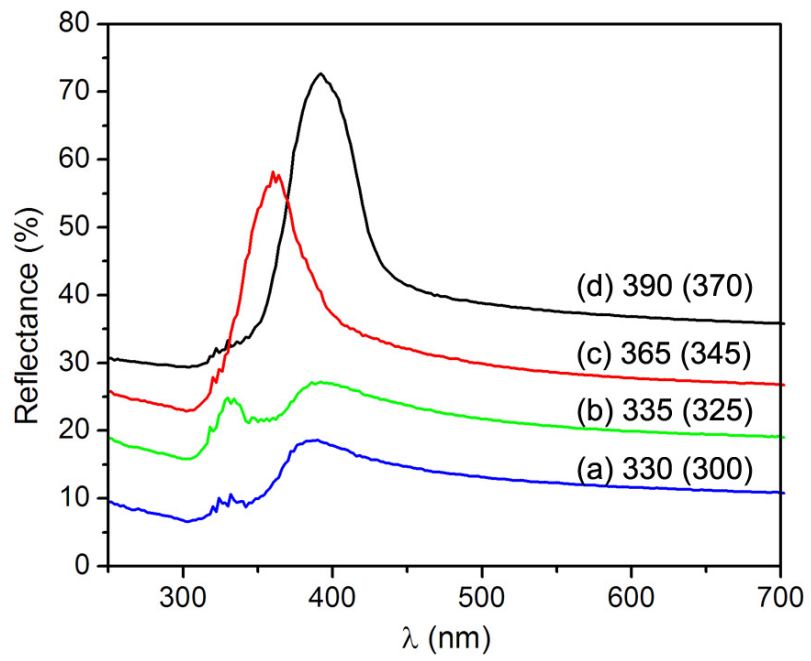

Figure 5.4. Reflectance spectra of $\mathrm{i}$-nc- $\mathrm{TiO}_{2}-\mathrm{o}$ measured in water after 30 minutes of $\mathrm{TiF}_{6}{ }^{2-}$ treatment with template sphere sizes of $130 \mathrm{~nm}$ (a), $150 \mathrm{~nm}$ (b), $180 \mathrm{~nm}$ (c) and $210 \mathrm{~nm}$ (d). The stop-band positions of the original i-nc- $\mathrm{TiO}_{2}-\mathrm{o}$ (in bracket) and after $\mathrm{TiF}_{6}{ }^{2-}$ treatment are labeled.

\subsection{Photocatalysis in Solution Phase}

\subsubsection{Effects of Surface Area and Diffusion}

Solution photocatalysis was carried out using methylene blue in $\mathrm{pH} 8$ water. Since surface area can influence the rate of photodegradation, with higher surface area giving 
more reactive sites and therefore higher efficiency, it is necessary to isolate this effect. Therefore, the accessible surface area to the dye was determined by an adsorptiondesorption experiment, as alternative methods such as $\mathrm{N}_{2}$ adsorption or ellipsometry were not feasible due to limited sample size and the slightly scattering nature of i-nc- $\mathrm{TiO}_{2}-\mathrm{o}$ films. The amount of dye adsorbed was determined spectroscopically after desorbing the dye in acidic water. The accessible area of various i-nc- $\mathrm{TiO}_{2}-\mathrm{o}$ is summarized in Table 1 , where a flat area of $1.2 \mathrm{~nm}^{2}$ was assumed for a methylene blue dye molecule. ${ }^{[14,15]}$ The amount of dye adsorbed depends on the concentration of negatively charged sites and the surface area of the $\mathrm{TiO}_{2}$ particle per unit mass. Since the surface chemistry between the samples is the same and therefore similar charge density can be expected, the amount of dye adsorbed can be interpreted as directly proportional to the surface area of the films. The specific surface area and weight of i-nc- $\mathrm{TiO}_{2}-\mathrm{o}$ of different sphere sizes are all comparable (3.0 $-3.5 \times 10^{-3} \mathrm{~m}^{2}$ for a film of $1 \times 2 \mathrm{~cm}$ in dimension), likely attributed to the similar filling fraction of $\mathrm{TiO}_{2}$ regardless of the template sphere size. The nc- $\mathrm{TiO}_{2}$ film of the same dimension has similar surface area but a much larger mass due to the dense packing of the nanocrystals. From table 5.1, the dye-accessible surface area per gram of inc- $\mathrm{TiO}_{2}-\mathrm{o}$ is $\sim 10 \mathrm{~m}^{2} / \mathrm{g}$, whereas that of $\mathrm{nc}-\mathrm{TiO}_{2}$ is $2.7 \mathrm{~m}^{2} / \mathrm{g}$. In comparison, $\mathrm{N}_{2}$ adsorption method suggests a surface area of $36 \mathrm{~m}^{2} / \mathrm{g}$ for nc- $-\mathrm{TiO}_{2}$, about 10 times higher than that determined by methylene blue adsorption. Therefore the estimated total surface area of inc- $\mathrm{TiO}_{2}-\mathrm{o}$, if determined using $\mathrm{N}_{2}$ adsorption, should be $\sim 100 \mathrm{~m}^{2} / \mathrm{g}$. So when normalized to weight, the $\mathrm{i}-\mathrm{nc}-\mathrm{TiO}_{2}-\mathrm{o}$ are actually 3-4 times higher in surface area than $\mathrm{nc}-\mathrm{TiO}_{2}$. To rule out the effect of differences in the number of active sites, all photocatalytic data shown below are normalized to the surface area rather than mass. 
Table 5.1. Mass and specific surface area of $\mathrm{i}-\mathrm{nc}-\mathrm{TiO}_{2}-\mathrm{o}$ before and after $\mathrm{TiF}_{6}{ }^{2-}$ treatment.

\begin{tabular}{|c|c|c|c|c|c|c|}
\hline & \multicolumn{5}{|c|}{ Template sphere size } & \multirow[b]{2}{*}{ nc- $\mathrm{TiO}_{2}$} \\
\hline & 130 & 150 & 180 & 210 & disordered & \\
\hline & \multicolumn{5}{|c|}{ As-prepared i-nc- $\mathrm{TiO}_{2}-\mathrm{O}$} & \\
\hline mass (mg) & $0.31 \pm 0.01$ & $0.29 \pm 0.08$ & $0.28 \pm 0.04$ & $0.34 \pm 0.04$ & $0.30 \pm 0.04$ & $1.3 \pm 0.2$ \\
\hline $\begin{array}{l}\text { accessible } \\
\text { area } \\
\left(\times 10^{-3} \mathrm{~m}^{2}\right)\end{array}$ & $3.4 \pm 0.1$ & $3.4 \pm 0.2$ & $3.36 \pm 0.05$ & $3.0 \pm 0.6$ & $3.1 \pm 0.4$ & $3.49 \pm 0.08$ \\
\hline \multicolumn{7}{|c|}{ After $\mathrm{TiF}_{6}{ }^{2-}$ treatment } \\
\hline $\begin{array}{l}\text { accessible } \\
\text { area } \\
\left(\times 10^{-3} \mathrm{~m}^{2}\right)\end{array}$ & $2.9 \pm 0.6$ & & & $3.4 \pm 0.3$ & $3.0 \pm 0.3$ & \\
\hline
\end{tabular}

\subsubsection{Rate of Degradation by As-Prepared Inverse $\mathrm{TiO}_{2}$ Opal}

Figure 5.5 shows the rate constants (normalized by the dye accessible area) for nc- $\mathrm{TiO}_{2}$, i-nc- $\mathrm{TiO}_{2}$-o of different sphere sizes and disordered i-nc- $\mathrm{TiO}_{2}-\mathrm{o}$. For solution photocatalysis, slow-photon enhancement is anticipated for 130 and 150-nm templated inc- $\mathrm{TiO}_{2}-\mathrm{O}$ as their photonic stop bands are at $300-325 \mathrm{~nm}$, the optimal energy range under white light irradiation as determined previously in Chapter 3. However, all of the as-prepared $\mathrm{i}-\mathrm{nc}-\mathrm{TiO}_{2}-\mathrm{o}$ show comparable rates at 1.5 times higher than $\mathrm{nc}-\mathrm{TiO}_{2}$. This general increase may be attributed to the increased diffusion rate within the porous structure of i-nc- $\mathrm{TiO}_{2}-\mathrm{o}$. Unexpectedly, the effect of slow photon was not at all detected in as-prepared i-nc- $\mathrm{TiO}_{2}$-o templated by $130 \mathrm{~nm}$ sphere, as its rate constant is comparable to $210 \mathrm{~nm}$ templated and disordered $\mathrm{i}$-nc- $\mathrm{TiO}_{2}-\mathrm{o}$. In addition, $\mathrm{i}$-nc- $\mathrm{TiO}_{2}-\mathrm{o}$ templated by 150 and $180 \mathrm{~nm}$ spheres show a lower degradation efficiency than the other inverse opals, possibly due to their stop-band reflections at 325 and $350 \mathrm{~nm}$, respectively, in water. From theoretical modeling, the slow-photon effect is weak due to the low refractive index contrast between the porous $\mathrm{TiO}_{2}$ wall and the aqueous-filled voids. Its effect, however, should be more pronounced in i-nc- $\mathrm{TiO}_{2}-\mathrm{o}$ with higher filling fraction. 


\subsubsection{Rate of Degradation by Inverse $\mathrm{TiO}_{2}$ Opals with Higher Filling Fraction}

To investigate slow-photon enhancement and the effect of $\mathrm{TiO}_{2}$ filling fraction independent of other factors, the photocatalytic rate of three specific $\mathrm{i}$-nc- $\mathrm{TiO}_{2}$-o were studied: that templated with (i) $130 \mathrm{~nm}$ spheres; (ii) $210 \mathrm{~nm}$ spheres and (iii) disordered opal. Samples (ii) and (iii) act as reference samples since they do not have photonic properties in the 300-350 $\mathrm{nm}$ range but possess similar porosity and surface area as (i). Note that the deposition of additional $\mathrm{TiO}_{2}$ by $\mathrm{TiF}_{6}{ }^{2-}$ is directly seeded by nanocrystals of as-prepared i-nc- $\mathrm{TiO}_{2}-\mathrm{o}$. Therefore samples with same porosity and surface area can be expected to have similar amount of increase in $\mathrm{TiO}_{2}$ filling fraction under the same reaction conditions. Interestingly after $\mathrm{TiF}_{6}{ }^{2-}$ treatment, the surface area did not change much even though the $\mathrm{TiO}_{2}$ loading increased (Table 1). With the increase in wall thickness and densification between the nanocrystals in $\mathrm{i}-\mathrm{nc}-\mathrm{TiO}_{2}-\mathrm{o}$, it is plausible that surface area does not increase in parallel with $\mathrm{TiO}_{2}$ loading.

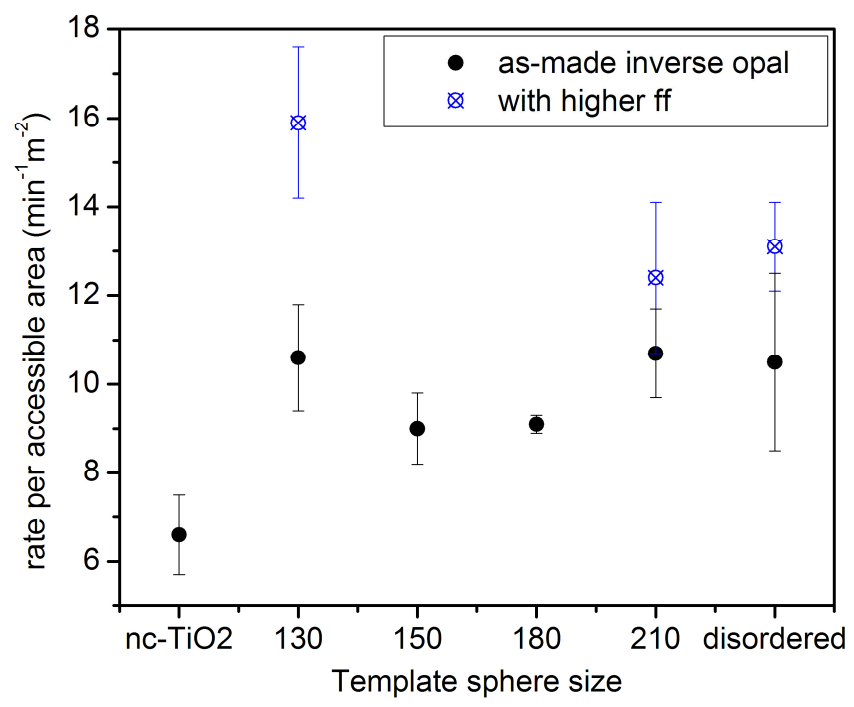

Figure 5.5. Rate of solution photocatalysis normalized with respect to the dye accessible area of various films: nc- $\mathrm{TiO}_{2}$, as-prepared $\mathrm{i}-\mathrm{nc}-\mathrm{TiO}_{2}-\mathrm{o}$ and those after increasing the $\mathrm{TiO}_{2}$ wall thickness. 
Figure 5.5 shows that the $\mathrm{TiF}_{6}{ }^{2-}$ treatment increased the photocatalytic efficiency for all three samples. The greatest increase is observed for $\mathrm{i}-\mathrm{nc}-\mathrm{TiO}_{2}-\mathrm{o}$ with $130-\mathrm{nm}$ spheres, whose degradation rate is now 1.3 times higher than $210-\mathrm{nm}$ and disordered inc- $\mathrm{TiO}_{2}-\mathrm{o}$ after $\mathrm{TiF}_{6}{ }^{2-}$ treatment. As all factors were held constant, the additional gain in the photodegradation efficiency of 130 -nm templated $\mathrm{i}$-nc- $\mathrm{TiO}_{2}$-o can be attributed to the slow-photon effect brought on by the higher refractive index contrast. Although theory suggests EF could be as high as 2 if $70 \%$ of the walls were made of $\mathrm{TiO}_{2}$, such a high densification is not really achievable in the current method given that the overgrowth of the $\mathrm{TiO}_{2}$ walls can close the interconnecting windows between the voids in the inverse opal, thus creating a non-closed packed fcc structure. SWA modeling suggests that the reduction in group velocity for a non-closed packed fcc structure is less than that of a cubic-closed packed structure; therefore closing the interconnecting pores is not beneficial for increasing the slow photon effect. For the modest increase of filling fraction presented here ( $\sim 50$ vol\% of $\mathrm{TiO}_{2}$ in the wall after $\mathrm{TiF}_{6}{ }^{2-}$ treatment), the observed increase of $\sim 30 \%$ in photocatalytic efficiency seems reasonable in comparison to the theoretical calculation. Another factor that is inherent in a solution system is the dispersive nature of light propagation. As the slow-photon effect is angle-dependent, light impinging on the film after traveling through the solution $(\sim 2 \mathrm{~cm})$ may have a much larger cone of irradiation, thus diluting the effect of orthogonal light. Nevertheless, the finding demonstrates the feasibility of increasing the slow-photon enhancement by increasing the filling fraction of $\mathrm{TiO}_{2}$. 


\subsection{Conclusions}

The various factors that contribute to the higher solution phase photocatalytic efficiency of $\mathrm{i}-\mathrm{nc}-\mathrm{TiO}_{2}-\mathrm{o}$ were investigated. By weight, $\mathrm{i}-\mathrm{nc}-\mathrm{TiO}_{2}-\mathrm{o}$ was shown to have four times the surface area as nc- $\mathrm{TiO}_{2}$, providing a greater number of active sites for degradation of molecules in solution. In addition, the macroporous structure of $\mathrm{i}$-nc- $\mathrm{TiO}_{2}$ o facilitates the diffusion of molecules. The amount of slow-photon enhancement under these conditions, although limited, can be increased by boosting the filling fraction of $\mathrm{TiO}_{2}$ in $\mathrm{i}-\mathrm{nc}-\mathrm{TiO}_{2}-\mathrm{o}$. For applications in water treatment, $\mathrm{i}$-nc- $\mathrm{TiO}_{2}-\mathrm{o}$ presents many fold higher efficiency due to its physical structure. However until highly densified $\mathrm{TiO}_{2}$ inverse opal framework can be achieved to bring about significant slow-photon enhancement, it is less crucial to have optimum stop-band energy or even an ordered structure.

\subsection{References}

[1] A. V. Emeline, V. K. Ryabchuk, N. Serpone, Catal. Today 2007, 122, 91.

[2] J. C. Yu, X. Wang, X. Fu, Chem. Mater. 2004, 16, 1523.

[3] F. Iskandar, A. B. D. Nandiyanto, K. M. Yun, C. J. Hogan, K. Okuyama, P. Biswas, Adv. Mater. 2007, 19, 1408.

[4] M. Alvaro, C. Aprile, M. Benitez, E. Carbonell, H. Garcia, J. Phys. Chem. B 2006, $110,6661$.

[5] Y. Sakatani, D. Grosso, L. Nicole, C. Boissiere, G. J. D. A. A. Soler-Illia, C. Sanchez, J. Mater. Chem. 2006, 16, 77.

[6] J. C. Yu, L. Zhang, Z. Zheng, J. Zhao, Chem. Mater. 2003, 15, 2280. 
[7] M. A. Carreon, S. Y. Choi, M. Mamak, N. Chopra, G. A. Ozin, J. Mater. Chem. 2007, 17, 82.

[8] C. C. Chen, X. Z. Li, W. H. Ma, J. C. Zhao, H. Hidaka, N. Serpone, J. Phys. Chem. B 2002, 106, 318.

[9] S. Lakshmi, R. Renganathan, S. Fujita, J. Photochem. Photobio. A 1995, 88, 163.

[10] A. Mills, J. S. Wang, J. Photochem. Photobio. A 1999, 127, 123.

[11] S. Nishimura, A. Shishido, N. Abrams, T. E. Mallouk, Appl. Phys. Lett. 2002, 81, 4532.

[12] S. Deki, Y. Aoi, J. Mater. Res. 1998, 13, 883.

[13] H. Kishimoto, K. Takahama, N. Hashimoto, Y. Aoi, S. Deki, J. Mater. Chem. 1998, 8, 2019.

[14] G. M. Chen, J. J. Pan, B. X. Han, H. Yan, J. Disp. Sci. Tech. 1999, 20, 1179.

[15] P. V. Sivapullaiah, B. Guru Prasad, M. M. Allam, Geotech. Test. J. 2008, 31, GTJ101429. 


\title{
CHAPTER 6
}

\section{Synergy of Slow Photon and Chemically Amplified Photochemistry in}

\section{Platinum-loaded Inverse Titania Opals}

(Reproduced in part with permission from J. Am. Chem. Soc. 2008, 130, 5420. Copyright 2008 American Chemical Society)

\begin{abstract}
While the field of photonic crystals has matured, there is an increasing need to apply the optical phenomena found only in designed photonic architectures to widespread photoprocesses like photovoltaics and photocatalysis. On the other hand, there are extensive resources on the chemical modifications of existing materials in order to improve the beneficial properties for technological importance. The potential to bring photoprocesses to new heights through the union of optical and chemical enhancements, however, has not been demonstrated to date. As a result, a study aimed at demonstrating such cooperativity was carried out. In this chapter, the synergy of slow photon optical amplification with chemical enhancement is demonstrated in inverse $\mathrm{TiO}_{2}$ opals loaded with Pt nanoclusters (i-Pt-TiO ${ }_{2}-\mathrm{o}$ ). By incorporating Pt nanoparticles on the surface of inverse $\mathrm{TiO}_{2}$ opals, more light is absorbed due to the slow photon effect while the lifetimes of the UV-excited electron and holes are extended by the Pt. A fourfold increase in the photodegradation efficiency was achieved.
\end{abstract}




\subsection{Introduction}

The metal-semiconductor junction is one of the most fundamental effects in solid state that has had great implications on industrial applications involving transistors or any other semiconductor device. The principles behind bulk metal-semiconductor interactions, such as the formation of the Schottky barrier and aligning of the Fermi level, apply also to a colloidal system but the small size and geometry of nanoparticles can additionally introduce interesting new properties. For example, band bending of the electronic states is negligible for $\mathrm{TiO}_{2}$ nanoparticles in contact with a metal or electrolyte as the particle size is much smaller than the width of the depletion layer. ${ }^{[1]}$ In colloidal metal- $\mathrm{TiO}_{2}$ systems, various metals, in particular nanoparticle forms of noble metals like $\mathrm{Au}, \mathrm{Pt}$ and $\mathrm{Ag}$, have been shown to enhance the separation of UV-excited electrons and holes. ${ }^{[2,3]}$ In these colloidal metal-semiconductor systems, the excited electrons in the conduction band of the semiconductor travels to the nanoparticle metal centers and are subsequently scavenged rapidly by $\mathrm{O}_{2}$ to form $\mathrm{O}_{2}^{-\bullet}$ (see Fig. 6.1). ${ }^{[4-7]}$ Note that since the electron diffusion time is on the order of $0.1 \mathrm{ps}$ for a $\sim 10 \mathrm{~nm}$ particle, the electron transfer from $\mathrm{TiO}_{2}$ to the metal island is rapid enough to compete with recombination processes. ${ }^{[8]}$

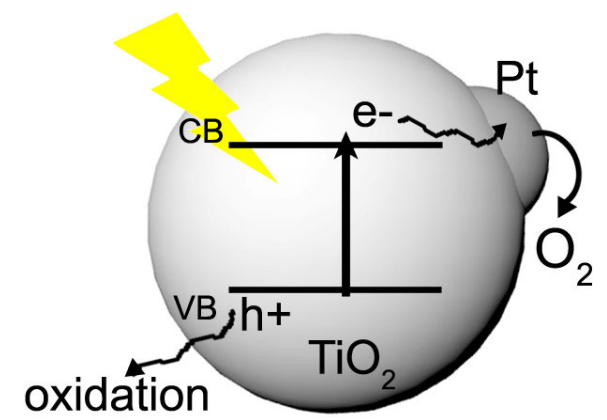

Figure 6.1. Schematic showing the pathways of the photogenerated electron and hole on a $\mathrm{TiO}_{2}$ particle whose surface is modified with Pt. 
Several researchers have studied the electron transfer process at the interface of metal-semiconductor nanoparticles. For example, photoinduced electron transfer from colloidal $\mathrm{ZnO}$ to surface-attached $\mathrm{Au}$ and $\mathrm{Ag}$ nanoparticles was detected by monitoring the plasmonic absorption wavelength (which is sensitive to the electron density) of the metal nanoparticles. ${ }^{[3]}$ The electron transfer from photoexcited $\mathrm{TiO}_{2}$ to metal was revealed by the changes in the photoactivity of $\mathrm{TiO}_{2}$, such as an increase in the photocurrent of a photoelectrochemical cell ${ }^{[9]}$ and a more efficient photocatalytic process for metal-loaded colloidal $\mathrm{TiO}_{2}$. Since there are various ways of depositing metal nanoparticles on colloidal $\mathrm{TiO}_{2}$, from impregnation, photodeposition ${ }^{[10]}$, thermal reduction of metal salts ${ }^{[11]}$ to pre-synthesizing ligand-capped metal nanoparticles, the chemical properties of the metal nanoparticles can be rather different leading to nonconsistent effects in earlier research. However, work has now established that metal nanoparticles like $\mathrm{Pt}$ can enhance the electron transfer from $\mathrm{TiO}_{2}$ to $\mathrm{O}_{2}$ and that the nature of the metal centers, especially the oxidation state, is very important. ${ }^{[12,13]}$

As the fabrication of $\mathrm{i}-\mathrm{nc}-\mathrm{TiO}_{2}$-o involves straightforward sol-gel chemistry using a colloidal crystal as template, it may be possible to integrate chemical modifications to bring the slow photon photocatalytic enhancement to further heights. Of the different metals, Pt has been found to be the most efficient for reducing the electron-hole recombination and is therefore adopted for the chemical modification to i-nc- $\mathrm{TiO}_{2}-\mathrm{o}$. Unlike $\mathrm{Au}$ or Ag, Pt lacks size-dependent surface plasmons due to the d-d damping effect, ${ }^{[14]}$ and should present less influence on the photonic properties. By incorporating Pt nanoparticles on the surface of $\mathrm{i}-\mathrm{nc}-\mathrm{TiO}_{2}-\mathrm{o}$, the lifetimes of the higher population of $\mathrm{UV}$-excited electron and holes arising from slow-photon enhanced absorption of $\mathrm{TiO}_{2}$ 
can be extended. The judicious control of the Pt loading through the photodeposition of $\mathrm{PtCl}_{6}{ }^{2-[10]}$ leads to a fourfold increase in the photodegradation efficiency.

\subsection{Experimental Methods}

\subsubsection{Fabrication of Pt-loaded Inverse $\mathrm{TiO}_{2}$ Opals}

The $\mathrm{i}-\mathrm{nc}-\mathrm{TiO}_{2}-\mathrm{o}$ were fabricated by using $185 \mathrm{~nm}$ spheres as templates (see Chapter 3). ${ }^{[15]}$ The disordered i-nc- $\mathrm{TiO}_{2}-\mathrm{O}$ was obtained from the binary template of 150 and $210 \mathrm{~nm}$ spheres with 0.2 fractions of $210 \mathrm{~nm}$ (Chapter 4). ${ }^{[16]}$ Chloroplatinic acid $\left(\mathrm{H}_{2} \mathrm{PtCl}_{6}\right)$ and methanol from Aldrich were used as received. For Pt photodeposition, a calcined film of $\mathrm{i}-\mathrm{TiO}_{2}$-o was immersed in a solution containing 50:50 volumes of water and methanol at $\mathrm{pH} 4$ (adjusted by $\mathrm{HCl}$ ) with $\mathrm{Pt}$ concentration varying from $1 \times 10^{-5}$ to $5 \times 10^{-4} \mathrm{M}$, and degassed under nitrogen for $30 \mathrm{~min}$. The $\mathrm{i}-\mathrm{nc}-\mathrm{TiO}_{2}-\mathrm{O}$ film suspended in the Pt solution was then irradiated by light with wavelengths greater than $300 \mathrm{~nm}$ (using a 120W Xe lamp and a cutoff filter) for $30 \mathrm{~min}$. The film was removed from the solution, rinsed with ethanol and dried under nitrogen.

\subsubsection{X-ray Photoelectron Spectroscopy}

X-ray photoelectron spectroscopy (XPS) of films on glass substrate was performed using Thermo Scientific K-Alpha XPS spectrometer with a spot area of 300 $\mu \mathrm{m}$. A monochromatic Al $\mathrm{K}_{\alpha} \mathrm{X}$-ray source was used and the spectra were shifted using adventitious carbon as the internal reference (binding energy of $285.0 \mathrm{eV}$ ). The peak fitting was performed by the software Avantage, using Shirley background and Lorentzian peaks. The amount of Pt loading was determined from the XPS results. 


\subsubsection{Dye Adsorption and Photodegradation}

Adsorption behavior of Acid Orange 7 was studied at $\mathrm{pH}$ 3. Photodegradation of adsorbed acid orange under white light irradiation was carried out using a 120W Xe lamp and a neutral density filter that cuts off $\lambda<300 \mathrm{~nm}$. Extinction spectra were taken at $30 \mathrm{~s}$ intervals over a total time of three minutes. No dye degradation was observed under visible light irradiation $(\lambda>400 \mathrm{~nm})$ in the time frame of the experiments.

\subsection{Photodeposition and Characterization of Pt-loaded Inverse $\mathrm{TiO}_{2}$ Opals}

$\mathrm{Pt}$ nanoparticles were photodeposited on $\mathrm{TiO}_{2}$ from a methanol/water mixture containing $\mathrm{PtCl}_{6}{ }^{2-}$ as starting complex. The photogenerated electron of $\mathrm{TiO}_{2}$ can reduce the platinum complex while the hole is scavenged by methanol to yield $\mathrm{CH}_{3} \mathrm{OH}^{\bullet+}$ radicals, which are also highly reducing. Studies have shown that most of the reducing power actually comes from the $\mathrm{CH}_{3} \mathrm{OH}^{\circ}$ radicals rather than the conduction band electrons of $\mathrm{TiO}_{2}{ }^{[17]}$ Hence methanol not only acts as a hole scavenger but also a reducing agent. On the other hand, the platinum complex undergoes hydrolysis readily to give $\mathrm{Pt}(\mathrm{OH}) \mathrm{Cl}_{5}{ }^{2-}$, $\mathrm{Pt}(\mathrm{OH})_{2} \mathrm{Cl}_{4}{ }^{2-}$ or $\mathrm{Pt}(\mathrm{OH})_{4} \mathrm{Cl}_{2}{ }^{2-}$ species. ${ }^{[18,19]}$ The rate of hydrolysis can be slowed down by making the solution acidic and indeed research has shown that the photodeposited product depends greatly on the $\mathrm{pH}$ of the solution. At neutral or basic condition, only $\mathrm{Pt}(\mathrm{OH})_{2}, \mathrm{PtO}$ and $\mathrm{PtO}_{2}$ have been photodeposited. However by lowering the $\mathrm{pH}$ to 3 , one can obtain nanoparticles of metallic Pt. These observations have been confirmed in a separate experiment; when the $\mathrm{pH}$ is not low enough, photodeposited oxides and hydroxides of $\mathrm{Pt}$ essentially serve as recombination centers on $\mathrm{TiO}_{2}$, dramatically decreasing the photodegradation efficiency. In the following sections, the 
characterization and properties of $\mathrm{Pt}$ clusters in $\mathrm{i}-\mathrm{Pt}-\mathrm{TiO}_{2}-\mathrm{O}$ obtained under acidic condition are presented.

\subsubsection{Morphological Study of Pt Clusters by STEM}

Figure 6.2a shows the SEM image of the ordered structure of $\mathrm{i}-\mathrm{Pt}-\mathrm{TiO}_{2}-\mathrm{O}$. Higher magnification using scanning transmission electron microscopy (STEM) in the Z-contrast mode reveals the $\mathrm{Pt}$ nanoclusters on the $\mathrm{TiO}_{2}$ framework (Fig. 6.2b - d). A significant fraction of the clusters exhibits anisotropic geometry, suggesting preferential orientation of $\mathrm{Pt}$ on $\mathrm{TiO}_{2}$ during the photodeposition process. Furthermore, the STEM images reveal changes in the size distribution of the Pt particles for different loading amount, as
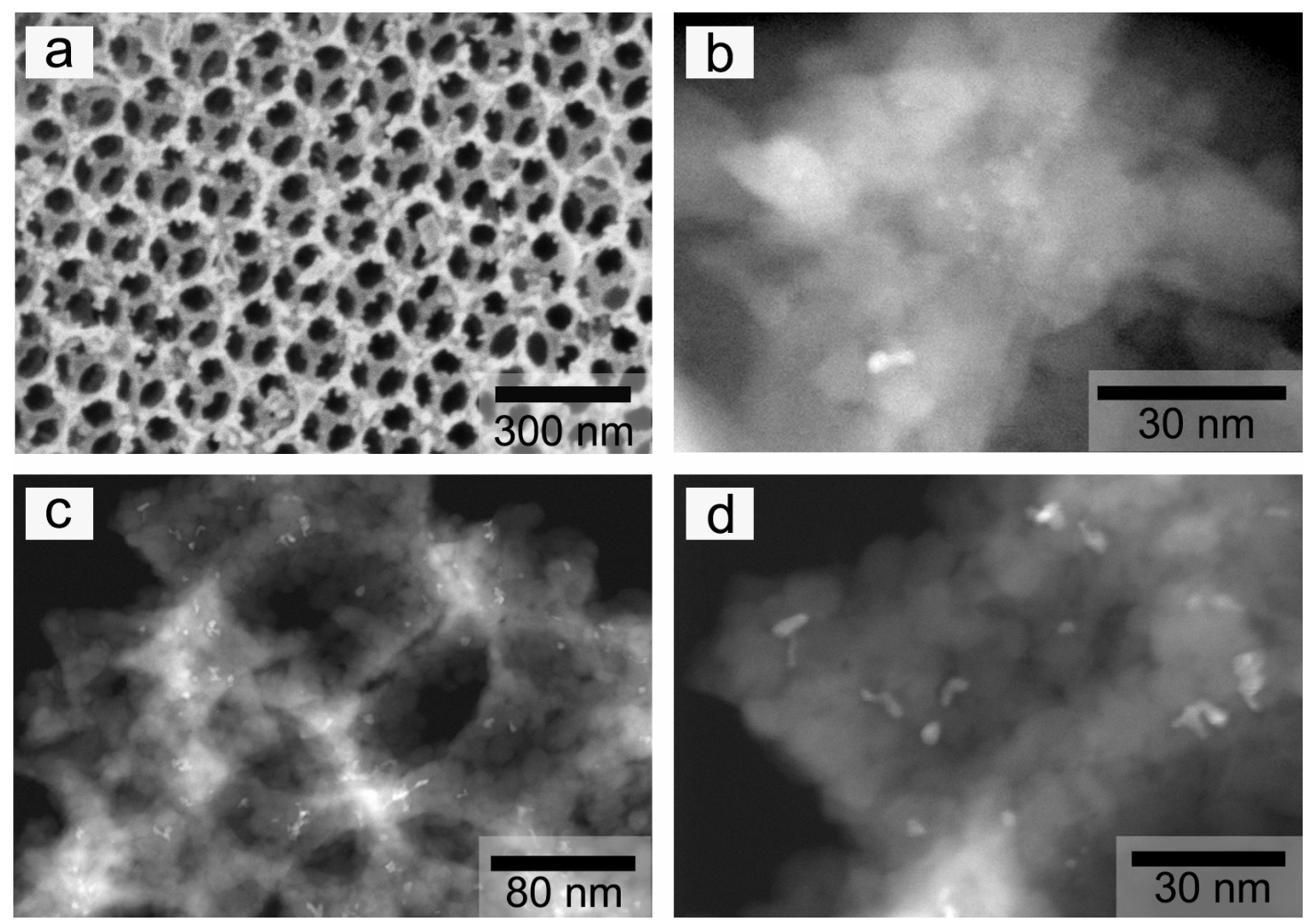

Figure 6.2. SEM image of $\mathrm{i}-\mathrm{Pt}-\mathrm{TiO}_{2}-\mathrm{o}(\mathrm{a})$, showing the ordered structure. STEM images of i-Pt$\mathrm{TiO}_{2}-\mathrm{o}$ with $1.3 \mathrm{wt} \%$ of $\mathrm{Pt}(\mathrm{b})$, and $2.7 \mathrm{wt} \%$ of $\mathrm{Pt}(\mathrm{c})$ and (d). 
summarized in Fig. 6.3. At $1.3 \mathrm{wt} \%$ the majority of the clusters are smaller than $1 \mathrm{~nm}$ but the bimodal distribution suggests a second peak around particle size of 2 to $2.5 \mathrm{~nm}$. Increasing the Pt loading to $2.3 \mathrm{wt} \%$ shows a decrease in the number of sub-nanometer clusters and an increase in the number of particles centered around $2.5 \mathrm{~nm}$. The average particle size increases from $1.5 \pm 0.8$ for $1.3 \mathrm{wt} \%$ Pt to $2.5 \pm 0.7 \mathrm{~nm}$ for $2.3 \mathrm{wt} \% \mathrm{Pt}$ loading. Further increase in Pt loading does not yield larger nanoparticles but merely increase the number of particles on the $\mathrm{TiO}_{2}$ framework.

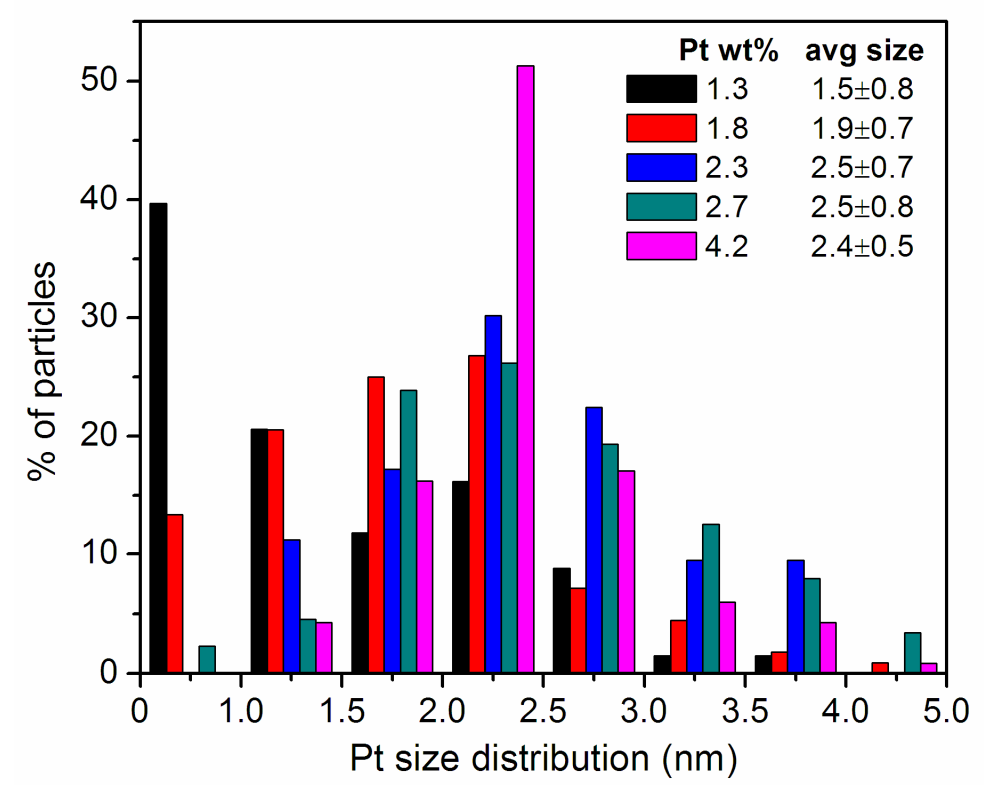

Figure 6.3. Size distribution of $\mathrm{Pt}$ nanoparticles on inverse $\mathrm{TiO}_{2}$ opals obtained from HR-STEM images. For simplicity, the dimension of a superficial spherical shape was used when determining the size of an irregularly shaped particle.

\subsubsection{Chemical Analysis by XPS}

XPS was employed to investigate the chemical nature of the Pt nanoparticles as the effect on photodegradation is greatly governed by the oxidation state of Pt. Metallic 
Pt facilitates electron transfer to $\mathrm{O}_{2}$ and thereby suppresses electron-hole recombination in $\mathrm{TiO}_{2}$, while higher oxidation states like $\mathrm{Pt}^{2+}$ or $\mathrm{Pt}^{4+}$ in hydroxide or oxide species may enhance recombination and have adverse effect on the photodegradation rate. ${ }^{[13]}$

Figure 6.4 shows the binding energies (B.E.) of $\mathrm{Pt} 4 f$ electrons in i-Pt-TiO ${ }_{2}-\mathrm{O}$ obtained from XPS. The analysis of pure i-nc- $\mathrm{TiO}_{2}$-o reveals loss structures at higher B.E. than the principle peaks in both Ti $2 p$ and $3 p$ spectra, ${ }^{[20]}$ with the latter overlapping the Pt $4 f$ signals. Therefore the Ti $3 p$ loss structure was included in the analysis of Pt $4 f$ signal as a single peak at $74.87 \mathrm{eV}$ (Fig. 6.5). The signals arising from different types of Pt species were deconvoluted; each signal comprises two peaks $\left(4 f_{7 / 2}\right.$ and $\left.4 f_{5 / 2}\right)$ as a result of the spin-orbit coupling. In general XPS shows two to three Pt species in i-Pt-TiO ${ }_{2}-\mathrm{o}$. At a loading of $1.3 \mathrm{wt} \%$, Pt $4 f_{7 / 2}$ peaks at B.E. 72.4 and $73.4 \mathrm{eV}$ were fitted (Fig. 6.4a) suggesting the formation of oxidized $\mathrm{Pt}^{2+}$ species like $\mathrm{Pt}(\mathrm{OH})_{2}$ and $\mathrm{PtO}$, whose reported
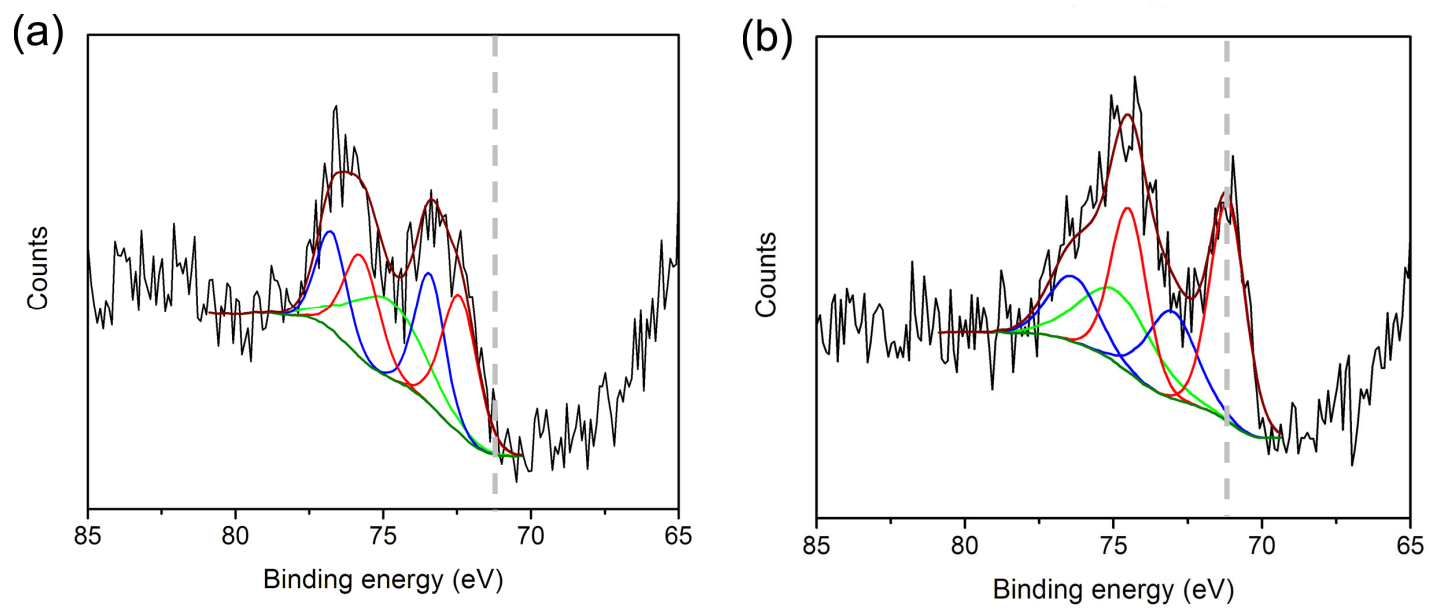

Figure 6.4. XPS spectra of Pt $4 f$ for $\mathrm{i}-\mathrm{Pt}-\mathrm{TiO}_{2}-\mathrm{o}$ with $1.3 \mathrm{wt} \% \mathrm{Pt}$ (a) and $2.7 \mathrm{wt} \% \mathrm{Pt}(\mathrm{b})$. Spinorbit coupling gives each $\mathrm{Pt}$ species 2 peaks $\left(4 f_{7 / 2}\right.$ and $\left.4 f_{5 / 2}\right)$ at $3.33 \mathrm{eV}$ apart; each pair of the fitted peaks is shown in the same color. A single peak at $74.87 \mathrm{eV}$ accounts for the loss structure of Ti $3 p$. The gray dashed line indicates the B.E. of metallic Pt $4 f_{7 / 2}$ orbital. 

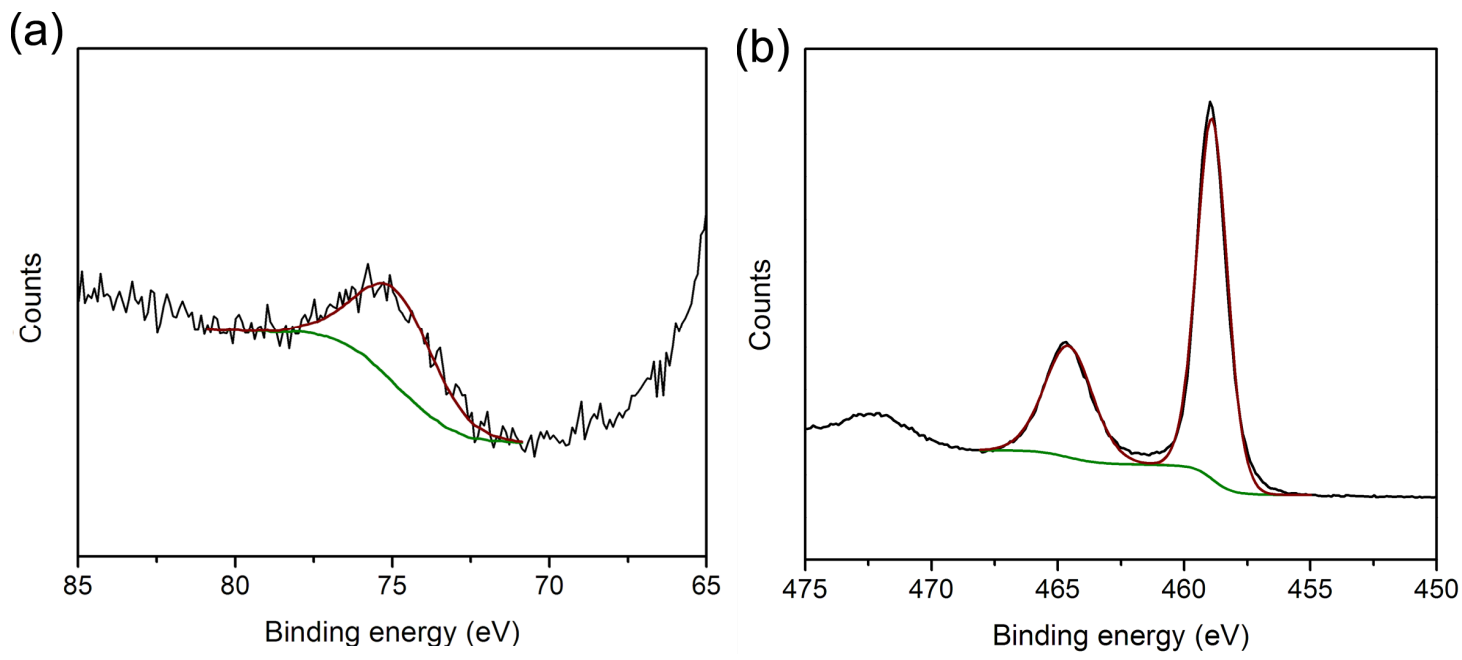

Figure 6.5. XPS spectra of the loss structure of $\mathrm{Ti} 3 p$ in $\mathrm{i}-\mathrm{TiO}_{2}-\mathrm{o}$ (a) and $\mathrm{Ti} 2 p$ in $2.7 \mathrm{wt} \% \mathrm{i}-\mathrm{Pt}-$ $\mathrm{TiO}_{2}-\mathrm{o}(\mathrm{b})$. The loss structure in (a) of $\mathrm{i}-\mathrm{TiO}_{2}-\mathrm{o}$ was fitted by a single peak at binding energy of $74.87 \mathrm{eV}$ with FWHM of $3 \mathrm{eV}$. The loss structure is taken into account in all Pt $4 f$ fittings by including this peak, with the peak area ratio fixed to the Ti $2 p$ signal of each sample. No change in the binding energy of $\mathrm{Ti}$ in $\mathrm{i}-\mathrm{Pt}-\mathrm{TiO}_{2}$-o was observed; a single $\mathrm{Ti}^{4+}$ with $2 p_{3 / 2}$ binding energy of $458.8 \mathrm{eV}$ was found for all samples.

B.E. are 72.6 and $73.8 \mathrm{eV}$ respectively. ${ }^{[21,22]}$ Since there are no ligand capping groups on the Pt nanoparticles, it is plausible that hydroxides and oxides cover the surface and make up all of the composition of sub-nanometer nanoparticles. Figure $6.4 \mathrm{~b}$ shows the $\mathrm{Pt} 4 f$ spectrum of i-Pt-TiO ${ }_{2}-\mathrm{O}$ with $2.7 \mathrm{wt} \% \mathrm{Pt}$. The fitted peaks have $4 f_{7 / 2}$ B.E. of 71.2 and $73.0 \mathrm{eV}$, corresponding to $\mathrm{Pt}^{0}$ and $\mathrm{Pt}^{2+}$ species. The main $4 f_{7 / 2}$ peak at $71.2 \mathrm{eV}$ suggests that by increasing $\mathrm{Pt}$ loading to $>2 \mathrm{wt} \%, \mathrm{Pt}^{0}$ is deposited as the particles grow in size.

Since these nanoparticles are high in surface area, hydroxides and oxides are still present even at higher Pt loadings. The fitted $4 f_{7 / 2}$ B.E. of $\mathrm{Pt}^{0}$ in all i-Pt- $\mathrm{TiO}_{2}-\mathrm{o}$ varies from 71.2 to $71.6 \mathrm{eV}$, close to the reported value of $71.2 \mathrm{eV}$ for bulk $\mathrm{Pt}^{\left[{ }^{[23]}\right.} \mathrm{A}$ B.E. shift 
from 71.2 to $71.5 \mathrm{eV}$ is suggested to occur for nanoparticles $2-5 \mathrm{~nm}$ in size, as the average coordination number of $\mathrm{Pt}$ is $10,{ }^{[11]}$ slightly lower than the bulk coordination number of 12. Due to contributions from other Pt species, the peak fitting is inconclusive about the extent of the quantum size effect. However the STEM and XPS results unambiguously demonstrate that nanoparticles of $\mathrm{Pt}$ were deposited on the $\mathrm{TiO}_{2}$ inverse opals, with a significant amount of $\mathrm{Pt}^{0}$ at higher loadings. Moreover, the oxidation state of $\mathrm{Ti}^{4+}$ is unchanged after the photodeposition.

\subsubsection{Optical Properties}

Figure 6.6 shows the reflectance and extinction spectra of inverse opal before and after Pt deposition. As can be seen, there is negligible change in the spectra, and the films also appear identical by eye. The photonic properties should therefore be comparable for i-Pt- $\mathrm{TiO}_{2}-\mathrm{o}$ and i-nc- $\mathrm{TiO}_{2}-\mathrm{o}$.
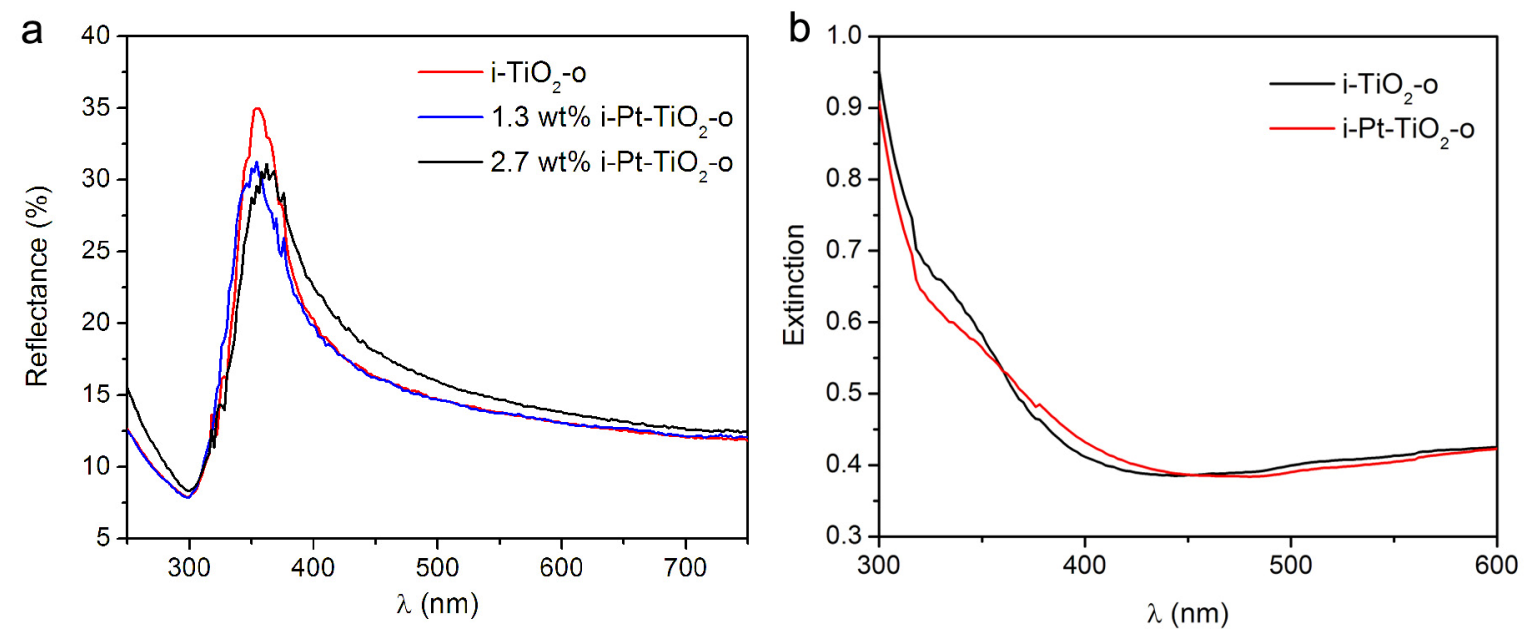

Figure 6.6. Reflectance spectra of $\mathrm{i}-\mathrm{TiO}_{2}-\mathrm{o}$ and $\mathrm{i}-\mathrm{Pt}-\mathrm{TiO}_{2}-\mathrm{o}$ in water (a) and extinction spectra in air before and after Pt photodeposition (b). 


\subsection{Adsorption of Acid Orange on Pt-loaded Inverse $\mathrm{TiO}_{2}$ Opals}

To investigate the photodegradation efficiency of $\mathrm{i}-\mathrm{Pt}-\mathrm{TiO}_{2}-\mathrm{o}$, the solid-state experiment employing acid orange as probe molecules was carried out. Methylene blue was not suitable for $\mathrm{i}-\mathrm{Pt}-\mathrm{TiO}_{2}-\mathrm{O}$ as the dye molecules tend to aggregate on the surface of the inverse opal when Pt is present. It may be due to the electrostatic interaction between positively charged methylene blue and the slightly negatively charged surface of $\mathrm{Pt}$ nanoparticles. On the contrary, acid orange chemisorbs on $\mathrm{TiO}_{2}{ }^{[24,25]}$ and in fact its adsorption behavior on pure $\mathrm{i}-\mathrm{nc}-\mathrm{TiO}_{2}-\mathrm{o}$ and $\mathrm{i}-\mathrm{Pt}-\mathrm{TiO}_{2}-\mathrm{o}$ was found to be similar. Because of its negative charge, acid orange adsorbs on $\mathrm{TiO}_{2}$ at low $\mathrm{pH}$. The adsorption isotherm in Fig. 6.7 shows saturation is achieved at equilibrium concentration of $6 \times 10^{-4} \mathrm{M}$, where monolayer coverage is obtained. In general there was a decrease of $15-30 \%$ in the amount of dye adsorbed after Pt deposition. This observation can be understood as a portion of the $\mathrm{TiO}_{2}$ surface is covered by Pt species, effectively reducing the number of
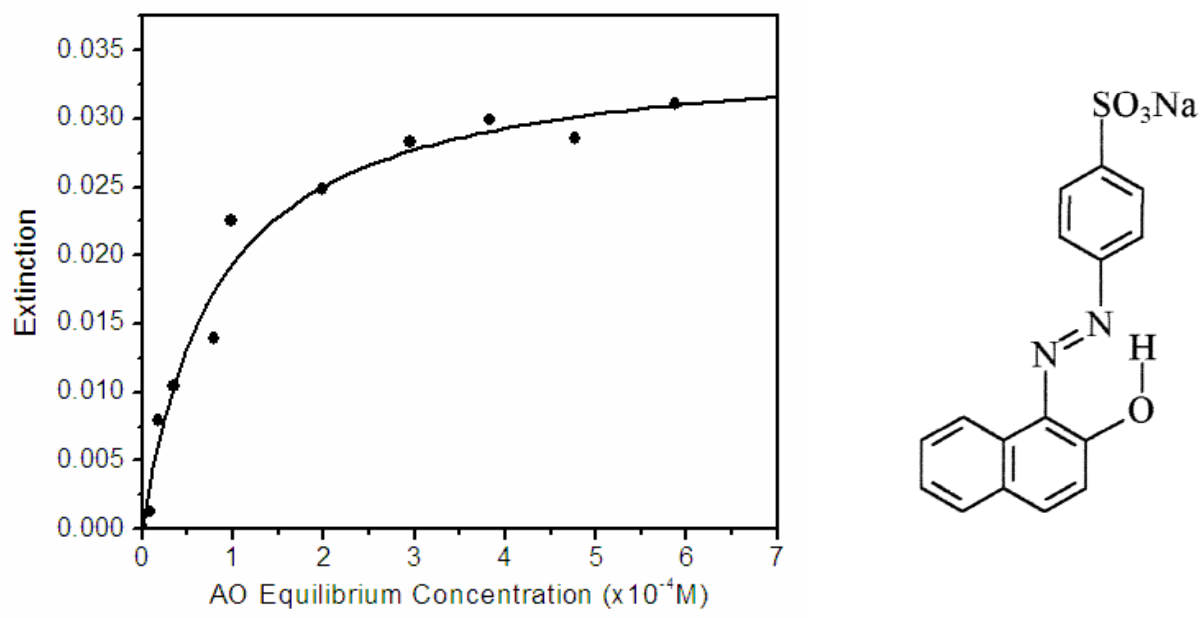

Figure 6.7. Adsorption isotherm of acid orange (structure shown on the right) on $\mathrm{i}-\mathrm{TiO}_{2}-\mathrm{o}$ at $\mathrm{pH} 3$. Saturation of dye adsorption is achieved at $6 \times 10^{-4} \mathrm{M}$ for all films. 
adsorption sites for the dye molecule. As the dye coverage was controlled to be monolayer for all films, the photodegradation experiment has been standardized with respect to the surface area of $\mathrm{TiO}_{2}$.

\subsection{Photodegradation Efficiency of Pt-loaded Inverse $\mathrm{TiO}_{2}$ Opals}

In order to test the hypothesis of cooperative amplification, the kinetics of photodegradation of acid orange on various samples were studied: i) nanocrystalline $\mathrm{TiO}_{2}$ $\left(\right.$ nc- $\left.\mathrm{TiO}_{2}\right)$ which serves as the reference sample; ii) $\mathrm{i}$-nc- $\mathrm{TiO}_{2}-\mathrm{o}$ with slow photon enhancement according to the previous work, and iii) i-Pt- $\mathrm{TiO}_{2}-\mathrm{o}$ of different $\mathrm{Pt}$ loadings derived from (ii). Figure 6.8a shows the logarithmic plot of relative extinction of acid orange on each sample as a function of irradiation time. First-order decay is observed and the decay rate constants are extrapolated and plotted in Fig. 6.8b. The enhancement factor, defined as the ratio of the decay rate constant of inverse opal to that of nc- $\mathrm{TiO}_{2}$, provides a clearer view of the amount of enhancement and is given as the right axis in Fig. $6.8 \mathrm{~b}$. The i-nc- $\mathrm{TiO}_{2}$-o fabricated from template spheres of $185 \mathrm{~nm}$ alone gives an $\mathrm{EF}$ of $\sim 1.7$, in agreement with the previous work (Chapter 3). The photodeposition of Pt on i-nc$\mathrm{TiO}_{2}-\mathrm{o}$ at $1.3 \mathrm{wt} \%$ initially did not yield any additional enhancement. As discussed above, low loadings of $\mathrm{Pt}$ lead to negligible amount of $\mathrm{Pt}^{0}$ deposition and the sub-nanometer clusters of Pt hydroxides and oxides species were not beneficial for facilitating UVexcited electron transfer to $\mathrm{O}_{2}$. However, a dramatic and abrupt increase in EF is observed when Pt loading reaches $\sim 2 \mathrm{wt} \%$, and then the enhancement only increases slightly with further increase of Pt loading. A similar trend had been observed for the oxidation of $\mathrm{CO}$ by Pt, where Heiz et al. suggested that a critical cluster size must be 
reached in order for the Fermi level of Pt to align or be above the $\mathrm{HOMO}$ of $\mathrm{O}_{2}$ for fast electron transfer. ${ }^{[26]}$ The significant increase in EF for compositions $>2 \mathrm{wt} \% \mathrm{Pt}$ can therefore be attributed to the presence of $\mathrm{Pt}^{0}$ that has grown beyond the critical cluster size. The $\mathrm{i}-\mathrm{Pt}-\mathrm{TiO}_{2}-\mathrm{O}$ with $\mathrm{Pt}$ loading up to $4 \mathrm{wt} \%$ resulted in an additional twofold chemical enhancement in comparison to $\mathrm{i}-\mathrm{TiO}_{2}-\mathrm{O}$. An overall $\mathrm{EF}$ of 4 was achieved with the highest Pt loading in i-Pt- $\mathrm{TiO}_{2}-\mathrm{O}$.
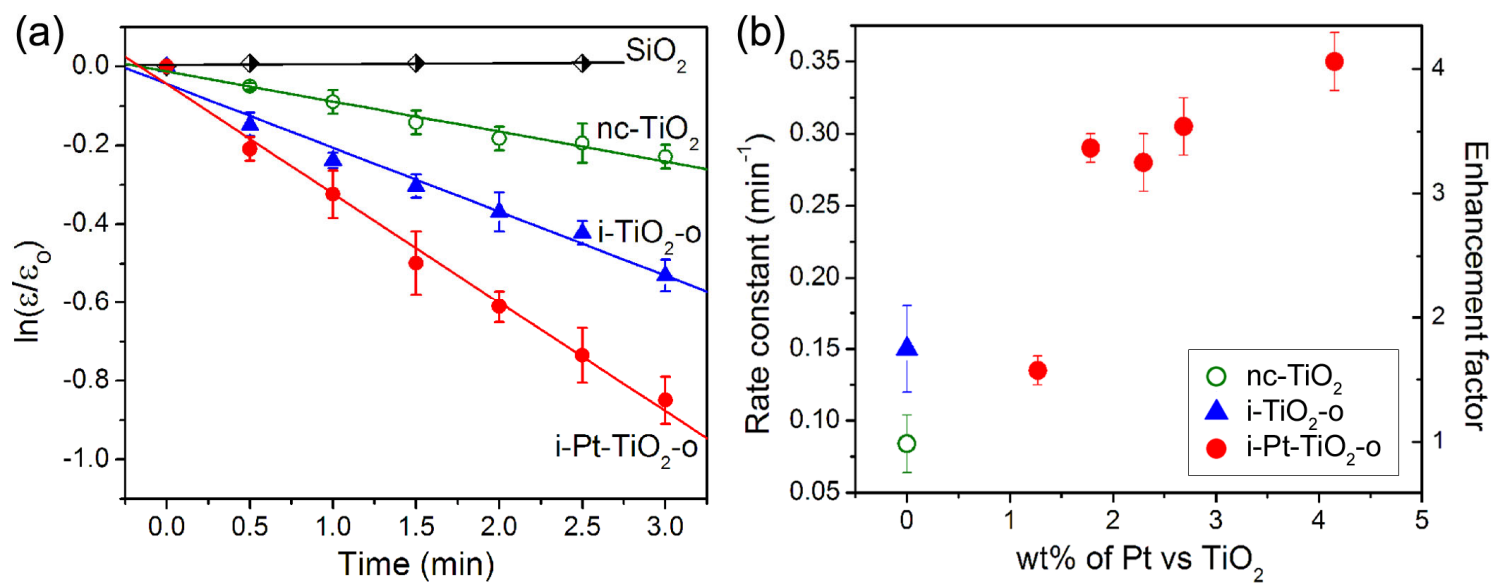

Figure 6.8. Photodegradation of acid orange under white-light irradiation. a) Logarithmic plot of relative dye extinction as a function of irradiation time for the blank $\left(\mathrm{SiO}_{2}\right), \mathrm{nc}-\mathrm{TiO}_{2}, \mathrm{i}-\mathrm{TiO}_{2}-\mathrm{o}$ and i-Pt-TiO ${ }_{2}$-o with $2.7 \mathrm{wt} \%$ Pt loading. b) First-order decay rate constant, also shown as the enhancement factor, of $\mathrm{i}-\mathrm{Pt}-\mathrm{TiO}_{2}-\mathrm{o}$ with different $\mathrm{Pt}$ loadings in comparison to $\mathrm{nc}-\mathrm{TiO}_{2}$.

In order to study the effect of $\mathrm{Pt}$ without the photonic effects, the photodegradation efficiency of disordered i-Pt- $\mathrm{TiO}_{2}-\mathrm{o}$ was examined. The disordered inc- $\mathrm{TiO}_{2}-\mathrm{o}$ was obtained according to Chapter 4 , with binary sphere composition chosen so that no photonic properties were observed. Such a disordered porous structure provides similar surface area and porosity, so that the photodeposition condition yielded a 
comparable loading of $\mathrm{Pt}$ as in an ordered inverse structure. The disordered $\mathrm{i}-\mathrm{nc}-\mathrm{TiO}_{2}-\mathrm{O}$ initially showed a comparable rate as $n c-\mathrm{TiO}_{2}$ without any $\mathrm{Pt}(\mathrm{EF}=0.8-1.1)$, but upon the incorporation of $2.5 \mathrm{wt} \% \mathrm{Pt}$, a maximum chemical enhancement of $76 \%$ resulted. This finding suggests that Pt alone cannot bring about the fourfold increase in the efficiency of the ordered i-Pt- $\mathrm{TiO}_{2}-\mathrm{o}$; there must exist both the slow-photon optical and the Pt-chemical enhancements. Quantitatively, the overall enhancement does not appear to be a direct additive effect of the two contributions, as the amount of the additional enhancement would be $150 \%$ ( $70 \%$ from slow photons and $80 \%$ from $\mathrm{Pt}$ ), or an $\mathrm{EF}$ of 2.5. Rather, the incorporation of $\mathrm{Pt}$ nearly doubles the rate of the initial $\mathrm{TiO}_{2}$ photocatalyst, such that disordered i-nc- $\mathrm{TiO}_{2}$-o with original EF close to 1 becomes 1.8 times more efficient with the presence of Pt, while ordered $\mathrm{i}-\mathrm{nc}-\mathrm{TiO}_{2}-\mathrm{o}$ with initial $\mathrm{EF}$ of 1.7 becomes $3.5-4$ times more efficient. Table 6.1 summarizes a comparison of rate constants for various samples demonstrating the synergistic effect of slow photons and Pt modification. The results suggest that the increase in the population of electrons and

Table 6.1. Comparison of rate constants for various samples demonstrating the synergistic effect of slow photons and Pt modification.

\begin{tabular}{|c|c|c|c|c|c|}
\hline & \multicolumn{4}{|c|}{ rate constant $k\left(\mathrm{~min}^{-1}\right)$} \\
\hline & & experimental & $\begin{array}{l}\Delta k, \\
\text { difference in } \\
\text { rate constant } \\
\text { with respect } \\
\text { to } 1\end{array}$ & $\begin{array}{c}\text { sum of } \\
\text { photonic }+\mathrm{Pt} \\
\text { enhancement } \\
(\Delta k \text { of } 2+3)\end{array}$ & $\begin{array}{c}\text { synergistic } \\
\text { enhancement } \\
\text { in } k \\
(\Delta k \text { of } 4-2-3)\end{array}$ \\
\hline 1 & $\mathrm{nc}-\mathrm{TiO}_{2}{ }^{\mathrm{a}}$ & 0.09 & & & \\
\hline 2 & ordered $\mathrm{i}-\mathrm{TiO}_{2}-\mathrm{O}^{b}$ & 0.15 & 0.06 & & \\
\hline 3 & $\begin{array}{l}\text { disordered } \mathrm{i}-\mathrm{Pt}-\mathrm{TiO}_{2}-\mathrm{O} \\
\text { with } 2.5 \mathrm{wt} \% \mathrm{Pt}^{\mathrm{c}}\end{array}$ & 0.17 & 0.08 & & \\
\hline 4 & $\begin{array}{l}\text { ordered i-Pt- } \mathrm{TiO}_{2}-\mathrm{O} \text { with } \\
\sim 2.5 \mathrm{wt} \% \mathrm{Pt}^{d}\end{array}$ & 0.30 & 0.21 & 0.14 & 0.07 \\
\hline
\end{tabular}


holes from the enhanced absorption does not impede the rapid electron transfer from $\mathrm{Pt}$ clusters to $\mathrm{O}_{2}$.

The hypothesis that slow photon is unaffected by Pt is further supported by scalarwave approximation modelling that suggests a negligible change in the group velocity of light at $<5 \mathrm{wt} \%$ of Pt. Figure 6.9 shows scalar wave approximation of the theoretical amplification factor as a function of $\mathrm{Pt}$ loading. The filling fraction of $\mathrm{TiO}_{2}$ in the walls of the inverse structure is modeled as $30 \%$, and the metallic $\mathrm{Pt}$ is incorporated in the wall as well. The bulk refractive index value of Pt was used for the modeling. At a Pt loading of $2-4 \mathrm{wt} \%$, the group velocity changed by less than $10 \%$ suggesting such low loadings of Pt should have minimal effect on slow photons. The overall enhancement of fourfold in i-Pt- $\mathrm{TiO}_{2}-\mathrm{o}$ is then likely the cooperative effect of slow photon and extended electronhole lifetime by the incorporation of Pt clusters.

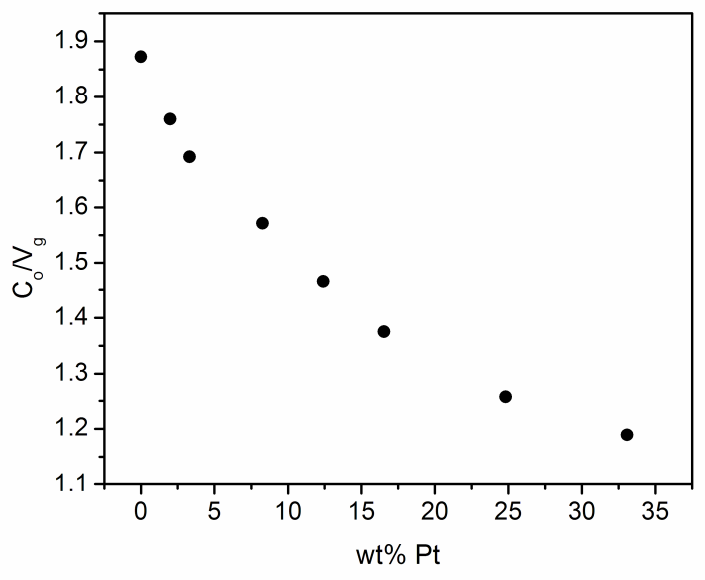

Figure 6.9. Scalar wave approximation of the theoretical amplification factor as a function of $\mathrm{Pt}$ loading. The bulk refractive index value of $\mathrm{Pt}$ is used for the modeling. At a Pt loading of 2 - 4 $\mathrm{wt} \%$, the group velocity changed by less than $10 \%$ suggesting such low loadings of Pt should have minimal effect on slow photons. 


\subsection{Conclusions}

The inverse $\mathrm{TiO}_{2}$ opal structure is versatile towards further chemical modifications like the incorporation of metal nanoparticles. By tuning photonic properties with respect to the anatase absorption and varying the amount of Pt loading on the surface of the anatase nanocrystals, a synergistic photochemical enhancement in $\mathrm{TiO}_{2}$ based inverse opals was realized. This proof of principle opens the door to the integration of different physical or chemical enhancements like slow photon amplified plasmonics that may prove extremely valuable in photochemistry and photocatalysis.

\subsection{References}

[1] J. S. Curran, D. Lamouche, J. Phys. Chem. 1983, 87, 5405.

[2] J. Disdier, J. M. Herrmann, P. Pichat, J. Chem. Soc., Faraday Trans. 1983, 79, 651.

[3] A. Wood, M. Giersig, P. Mulvaney, J. Phys. Chem. B 2001, 105, 8810.

[4] A. J. Bard, M. A. Fox, Acc. Chem. Res. 1995, 28, 141.

[5] M. A. Fox, M. T. Dulay, Chem. Rev. 1993, 93, 341.

[6] A. Fujishima, K. Honda, Nature 1972, 238, 37.

[7] N. Serpone, E. Perlizzetti, Photocatalysis: fundamentals and applications, John Wiley and Sons, New York 1989.

[8] M. Gratzel, Heterogeneous Photochemical Electron Transfer, CRC Press, Boca Raton, FL 1989.

[9] V. Subramanian, E. Wolf, P. V. Kamat, J. Phys. Chem. B 2001, 105, 11439.

[10] B. Kraeutler, A. J. Bard, J. Am. Chem. Soc. 1978, 100, 4317. 
[11] P. J. Chupas, K. W. Chapman, G. Jennings, P. L. Lee, C. P. Grey, J. Am. Chem. Soc. 2007, 129, 13822.

[12] G. R. Bamwenda, S. Tsubota, T. Nakamura, M. Haruta, Catal. Lett. 1997, 44, 83.

[13] J. Lee, W. Choi, J. Phys. Chem. B 2005, 109, 7399.

[14] P. Mulvaney, Langmuir 1996, 12, 788.

[15] J. I. L. Chen, G. von Freymann, S. Y. Choi, V. Kitaev, G. A. Ozin, Adv. Mater. 2006, 18, 1915 .

[16] J. I. L. Chen, G. von Freymann, V. Kitaev, G. A. Ozin, J. Am. Chem. Soc. 2007, $129,1196$.

[17] C. J. Xi, Z. S. Chen, Q. L. Li, Z. S. Jin, J. Photochem. Photobiol., A 1995, 87, 249.

[18] E. Blasius, W. Preetz, R. Schmitt, J. Inorg. Nuc. Chem. 1961, 19, 115.

[19] L. E. Cox, E. L. Wehry, D. G. Peters, J. Inorg. Nuc. Chem. 1972, 34, 297.

[20] M. A. Khan, A. Kotani, J. C. Parlebas, J. Phys.: Condens. Matter 1991, 3, 1763.

[21] J. E. Drawdy, G. B. Hoflund, S. D. Gardner, E. Yngvadottir, D. R. Schryer, Surf. Interface Anal. 1990, 16, 369.

[22] K. S. Kim, N. Winograd, R. E. Davis, J. Am. Chem. Soc. 1971, 93, 6296.

[23] M. G. Mason, Phys. Rev. B 1983, 27, 748.

[24] K. Bourikas, M. Stylidi, D. I. Kondarides, X. E. Verykios, Langmuir 2005, 21, 9222.

[25] C. Bauer, P. Jacques, A. Kalt, Chem. Phys. Lett. 1999, 307, 397.

[26] U. Heiz, A. Sanchez, S. Abbet, W. D. Schneider, J. Am. Chem. Soc. 1999, 121, 3214. 


\section{CHAPTER 7}

\section{Outlook and Future Directions}

\subsection{Application of Inverse $\mathrm{TiO}_{2}$ Opals in Sterilizing Light Bulbs}

The potential to amplify the photodegradation efficiency of $\mathrm{TiO}_{2}$ by slow photons has been explored in detail. While progress is still needed for applications in an aqueous environment (as discussed in Chapter 5), the use of inverse opals in air purification may be more promising as there exists no dielectric contrast problem involving gaseous phase photocatalysis. Preliminary work on this concept has been explored by Valsaraj and coworkers, in which the degradation of dichlorobenzene in a closed-loop reactor by i-nc$\mathrm{TiO}_{2}-\mathrm{O}$ under UV irradiation was investigated. ${ }^{[1]}$ They showed that i-nc- $\mathrm{TiO}_{2}-\mathrm{o}$ was more efficient than Degussa P25; however they did not elucidate the contribution of the different enhancement effects on the overall efficiency.

Of the various environments that require air purification, the indoors of buildings where daily activities are carried out have immediate importance to the public's health. This is especially true in hospitals. Since illumination provided by light bulbs contain a small fraction of UV light, it may be possible to use $\mathrm{TiO}_{2}$ to clean the air. ${ }^{[2]}$ In fact, fluorescent light bulbs coated with thin-film $\mathrm{TiO}_{2}$ are commercially available. Fresh ${ }^{2}$ claims that it can get rid of odors in a room when the consumer leaves the light on for a few hours. ${ }^{[3]}$ By making the $\mathrm{TiO}_{2}$ coating into an inverse opal, the efficiency could in theory increase due to the presence of slow photons of suitable energy. Moreover as described in Chapter 6 platinum nanoclusters could enhance the effectiveness of 
sterilization light bulbs even further. Therefore investigating the prospect of using i-nc$\mathrm{TiO}_{2}$-o for indoor air purification is technologically relevant and important.

\subsubsection{Gas-phase Photocatalysis Using Inverse $\mathrm{TiO}_{2}$ Opals and Incandescent Light Bulb}

The degradation of acetone by i-nc- $\mathrm{TiO}_{2}$-o under illumination of a household light bulb was investigated. Briefly, $5 \mu \mathrm{L}$ of acetone was injected into $20 \mathrm{~mL}$ septum-capped vials, which contained films of $\mathrm{i}-\mathrm{nc}-\mathrm{TiO}_{2}-\mathrm{o}$ or $\mathrm{nc}-\mathrm{TiO}_{2}$ on glass substrates. The concentration of acetone was monitored over time by gas chromatography (GC) using a gas-tight syringe as the sampling method. An empty vial containing $5 \mu \mathrm{L}$ of acetone under identical irradiation condition was used as the control; all GC signals were normalized accordingly. The experimental errors reflect the reproducibility of the GC measurements, rather than deviation of different films or experimental trials.

The amount of photodegradation of acetone by i-nc- $\mathrm{TiO}_{2}$-o of different template sphere sizes is summarized in Fig. 7.1. The efficiency of 345-i-nc- $\mathrm{TiO}_{2}$-o (prefix denotes the stop-band position in air, as in Chapter 3) was slightly higher than the other inverse opals; the enhancement likely arises from the red-edge slow-photon enhancement at 370$380 \mathrm{~nm}$, around the cut-off range of the UV component of the light bulb. On the other hand, the nc- $\mathrm{TiO}_{2}$ performed just as efficiently as $345-\mathrm{i}-\mathrm{nc}-\mathrm{TiO}_{2}-\mathrm{o}$.

\subsubsection{Prototype Light Bulb Coated with Inverse $\mathrm{TiO}_{2}$ Opal}

A method for depositing i-nc- $\mathrm{TiO}_{2}-\mathrm{o}$ on various types of light bulbs was developed and the prototypes are shown in Fig. 7.2. The photodegradation efficiency of the light bulb coated with i-nc- $\mathrm{TiO}_{2}-\mathrm{o}$ was compared to that dip-coated with nc- $\mathrm{TiO}_{2}$. 


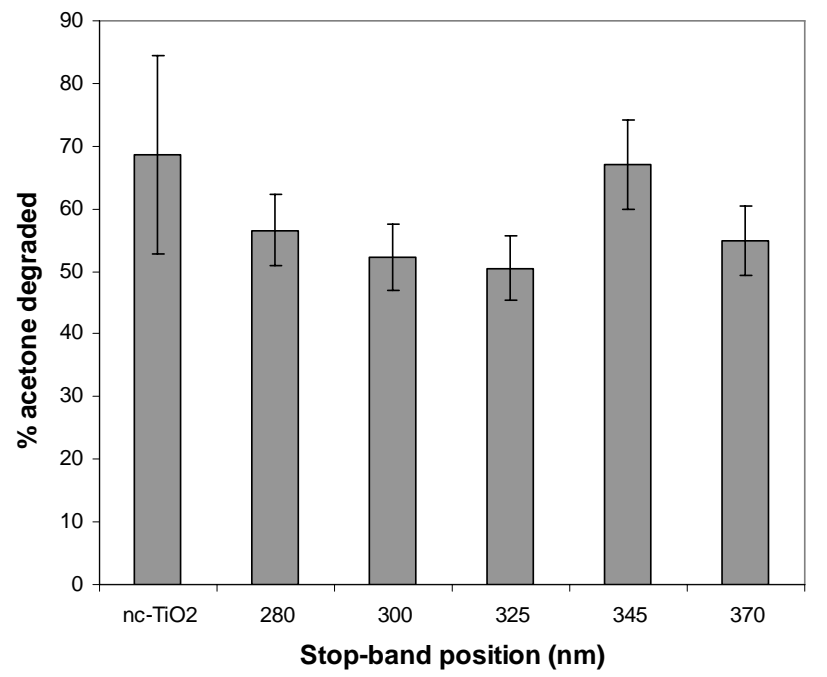

Figure 7.1. The percentage of acetone degraded by nc- $\mathrm{TiO}_{2}$ and $\mathrm{i}-\mathrm{nc}-\mathrm{TiO}_{2}-\mathrm{o}$ of different stopband positions after $50 \mathrm{~h}$ of irradiation using a 25W, $160 \mathrm{~V}$ incandescent light bulb.
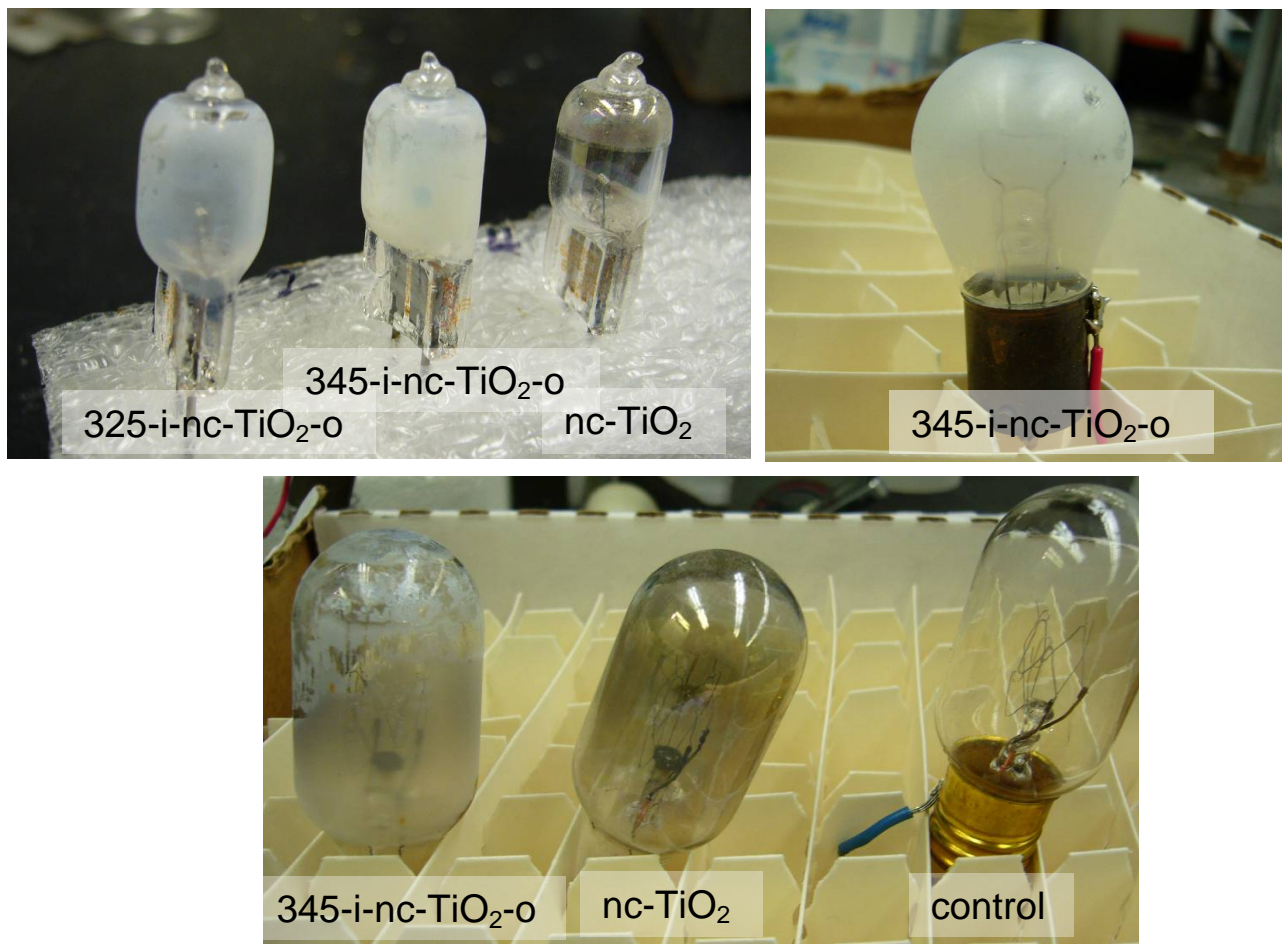

Figure 7.2. Photographs of various types of light bulbs coated with nc- $\mathrm{TiO}_{2}$ or i-nc- $\mathrm{TiO}_{2}-\mathrm{o}$. 
Figure 7.3 shows the custom-made chamber that was used for the photodegradation experiment; it consists of electrical connections and sampling outlets, from which air samples were taken and the signal from acetone was monitored periodically.

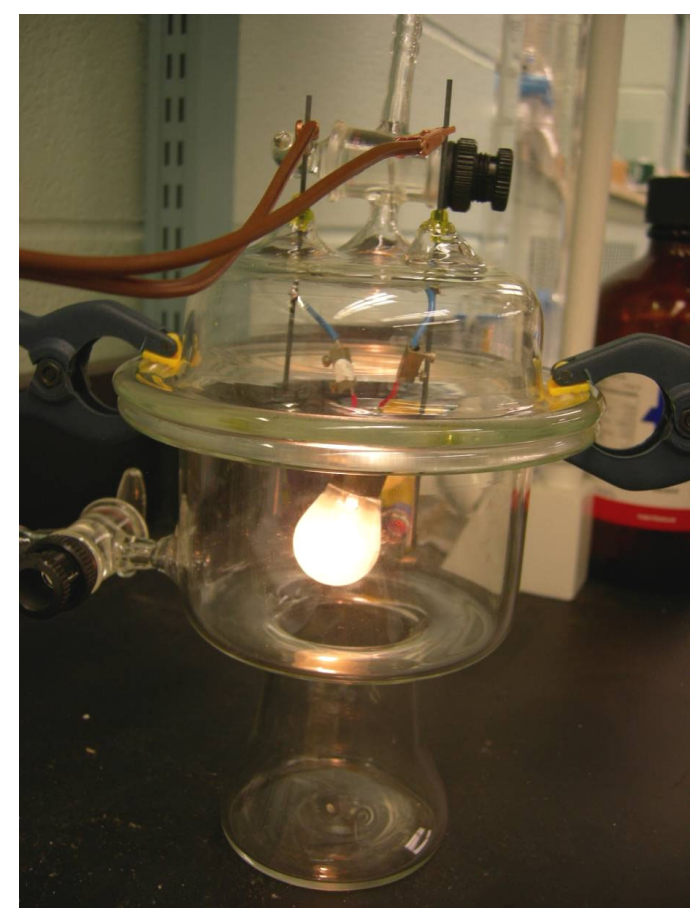

Figure 7.3. Photograph of the experimental set-up for studying the photodegradation efficiency of $\mathrm{TiO}_{2}$-coated light bulbs.

Figure 7.4 shows the changes in the amount of acetone as a function of illumination time using the functionalized light bulbs. From GC analysis, the degradation of acetone by both $\mathrm{TiO}_{2}$-coated light bulbs is relatively low, albeit faster than without the photocatalyst. However the rate of degradation by nc- $\mathrm{TiO}_{2}$ and $\mathrm{i}-\mathrm{nc}-\mathrm{TiO}_{2}-\mathrm{o}$ is entirely the same. Since the amount of nc- $\mathrm{TiO}_{2}$ on the light bulb was controlled to be similar to the inc- $\mathrm{TiO}_{2}-\mathrm{o}$, the comparison is normalized in terms of the mass. The macroporous structure 
of $\mathrm{i}$-nc- $\mathrm{TiO}_{2}$-o does not seem to offer enhancements in terms of the diffusion rate of molecules under the testing conditions, possibly a result of the relatively high concentration of acetone that is employed (for the ease of quantification with GC). In addition, the slow-photon amplification that was present in $345-\mathrm{i}-\mathrm{nc}-\mathrm{TiO}_{2}-\mathrm{o}$ on flat glass substrates is not detected in the light bulb experiment. This shortcoming may be due to the lower optical quality of the template opal on the curved surface of the light bulb. Moreover, the increase in photodegradation efficiency at $>370 \mathrm{~nm}$ may be limited by the low absorbance of $\mathrm{TiO}_{2}$ and the low quantum yield in this energy range. For applications using an incandescent light source, doped- $\mathrm{TiO}_{2}$ having extended visible absorption may be more promising. ${ }^{[4]}$

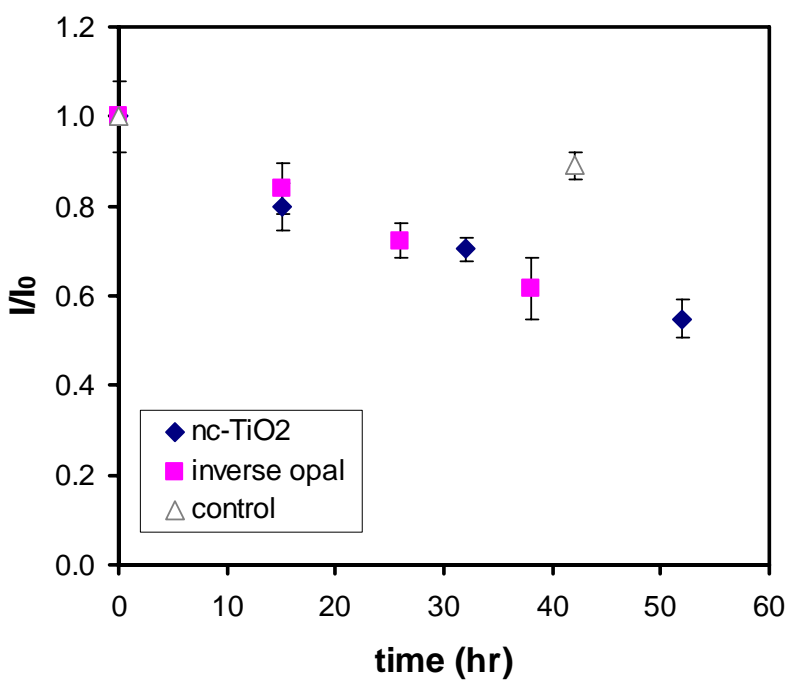

Figure 7.4. The relative intensity of acetone (measured by GC) as a function of illumination time for the light bulbs coated with nc- $\mathrm{TiO}_{2}$ or $345-\mathrm{i}-\mathrm{nc}-\mathrm{TiO}_{2}-\mathrm{o}$, or as is (control light bulb).

Several challenges need to be overcome for the use of $\mathrm{i}-\mathrm{nc}-\mathrm{TiO}_{2}$-o in indoor air purification. Due to the low fraction of UV light in the incandescent light source, a substantial amount of $\mathrm{TiO}_{2}$ is needed for achieving a notably high efficacy. While nc- 
$\mathrm{TiO}_{2}$ on a light bulb is readily attainable by dip-coating, with easy build-up in thickness by repeating the procedure, the making of $\mathrm{i}-\mathrm{nc}-\mathrm{TiO}_{2}-\mathrm{O}$ on the curved surface is challenging, constituting several stages, and the thickness of the film is not easily increased without the i-nc- $\mathrm{TiO}_{2}$-o peeling off. The highly porous inorganic framework of $\mathrm{i}$-nc- $\mathrm{TiO}_{2}$-o further poises the undesirable fragility to the coating; the mechanical instability against normal handling requires further modification to the constituents of the film. In conclusion, proof of concept and prototype i-nc-TiO ${ }_{2}$-o-coated light bulbs have been demonstrated, but continued efforts are needed to better understand the feasibility and commercial viability of such products.

\subsection{Other Photonic Structures for Optical Amplification}

\subsubsection{Tandem Layer Structure of Multiple Inverse $\mathrm{TiO}_{2}$ Opals}

The spectral window of the slow-photon enhancement in absorption is $\sim 40 \mathrm{~nm}$ for 300-i-nc- $\mathrm{TiO}_{2}$-o (Chapter 3). The energy range is larger than theoretical prediction but still rather limited. To increase the bandwidth of the enhanced absorption, inverse opals with different periodicities can be combined in series as stacks, creating a tandem layer structure. ${ }^{[5]}$ Each layer comprises of one $\mathrm{i}-\mathrm{nc}-\mathrm{TiO}_{2}-\mathrm{o}$, and the periodicity is varied between the layers so that the overall enhancement is the sum of the enhancements at different wavelengths of the individual layers. Figure 7.5 shows a bi-layer structure comprised of 325-i-nc- $\mathrm{TiO}_{2}-\mathrm{o}$ on top of 300-i-nc- $\mathrm{TiO}_{2}-\mathrm{o}$. High quality bi-layer i-nc- $\mathrm{TiO}_{2}-$ o can be obtained by sequentially depositing opals of different sphere sizes followed by the inversion process. However, while the individual layers are well ordered, the interface between the layers is rather disordered. It is unclear if such an interfacial layer creates 
photonic defects or affects the overall combination of the slow photons from different layers.

The photodegradation efficiency of the bi-layer inverse opal was examined; however no additional enhancement compared to 300-i-nc- $\mathrm{TiO}_{2}-\mathrm{o}$ was achieved. This observation may arise from the fact that $300-\mathrm{i}-\mathrm{nc}-\mathrm{TiO}_{2}-\mathrm{o}$ already absorbs as much of the light as possible; hence 325-i-nc- $\mathrm{TiO}_{2}$-o can offer very little additional absorption enhancement. As the absorption tail of $\mathrm{TiO}_{2}$ spans only from $320-380 \mathrm{~nm}$, the variation in the periodicity of the two layers is rather limited. Therefore, the tandem layer approach for enhancing the photodegradation efficiency of $\mathrm{TiO}_{2}$ may not be plausible. On the other hand, the layered structure should be promising for enhancing systems that have a wider absorption spectrum range, such as the dye-sensitized solar cells shown by Mihi and Miguez. ${ }^{[6]}$

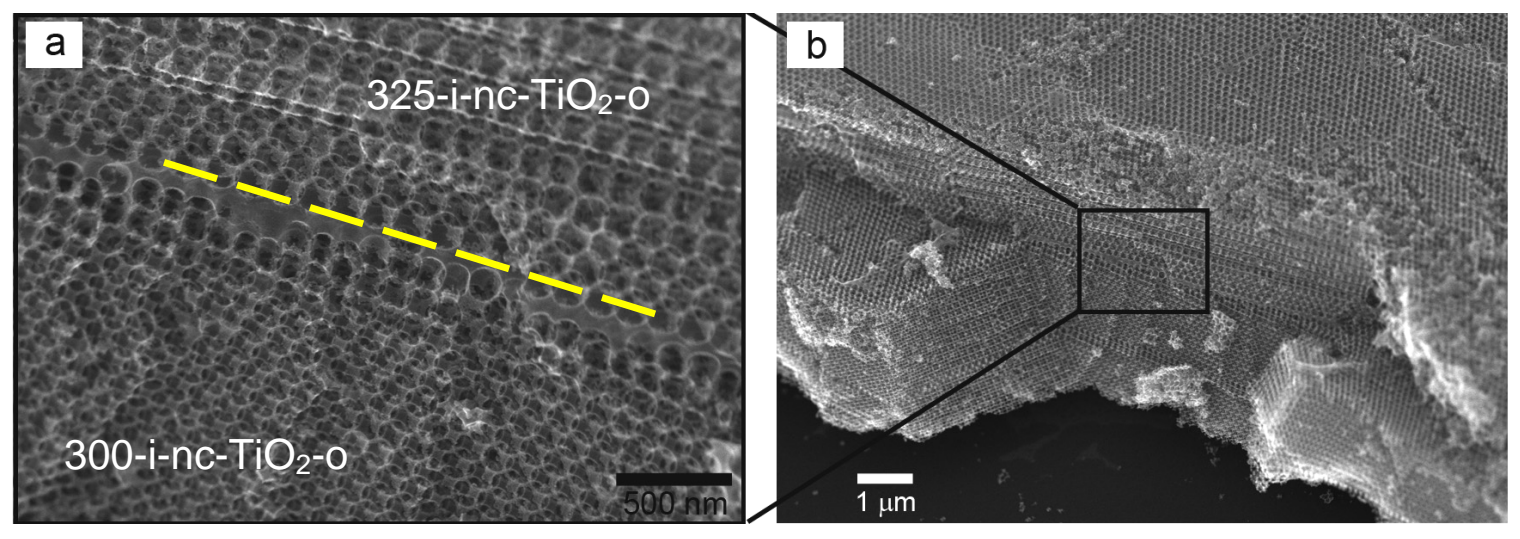

Figure 7.5. SEM images of the bi-layer structure with $325-\mathrm{i}-\mathrm{nc}-\mathrm{TiO}_{2}-\mathrm{o}$ on top of $300-\mathrm{i}-\mathrm{nc}-\mathrm{TiO}_{2}-\mathrm{o}$. The corresponding polystyrene template sphere sizes used were $180 \mathrm{~nm}$ and $150 \mathrm{~nm}$ respectively. The "defect" layer can be observed at the interface (outlined by the dashed line). 


\subsubsection{Bi-layer Structure Comprising a Non-absorbing Photonic Crystal}

Aside from photonic band edge effects, the stop bands of photonic crystals make them ideal spectral-selective reflectors, which have been shown to effectively increase the path length of light traveling through a non-structured absorbing layer. ${ }^{[7-9]}$ In such a bi-layer structure, the photonic crystal is placed at the back of the absorbing layer, reflecting wavelengths of interest, which did not get absorbed during the first path of light, back into the absorbing layer. This bi-layer concept poises several advantages over the slow-photon approach: the absorbing layer does not need to be structured into a photonic crystal; the spectral range of enhancement is wider than slow photons, as governed by the reflection of the photonic stop-band; and the photonic crystal can be made of any material, having the desired optical, and possibly other, properties. The tuning of the thickness of the absorbing surface layer can further bring on surfacelocalized modes that deflect light longitudinally rather than transversely as found in conventional mirroring effect.

A bi-layer structure of nc- $\mathrm{TiO}_{2}$ on $\mathrm{SiO}_{2}$ opal of $145 \mathrm{~nm}$ spheres is shown in Fig. 7.6. The stop band is positioned at $345 \mathrm{~nm}$ near the $\mathrm{TiO}_{2}$ absorption tail. The potential in enhancing this system is the highest when the amount of absorption is low, in other words when the $\mathrm{nc}-\mathrm{TiO}_{2}$ is very thin $(<100 \mathrm{~nm})$. In such a case, high reflectivity from the opal can be achieved. However this condition leads to the difficulty in carrying out the solidstate photodegradation method that has been used previously. There is a limited amount of dye adsorbed on nc- $\mathrm{TiO}_{2}$, the extinction of which was too low to be detected consistently by spectroscopy. Alternatively, the photoactivity of $\mathrm{TiO}_{2}$ can be derived from 
its photoconductivity, and such a study in the future on this bi-layer structure would help elucidate the photonic effects.
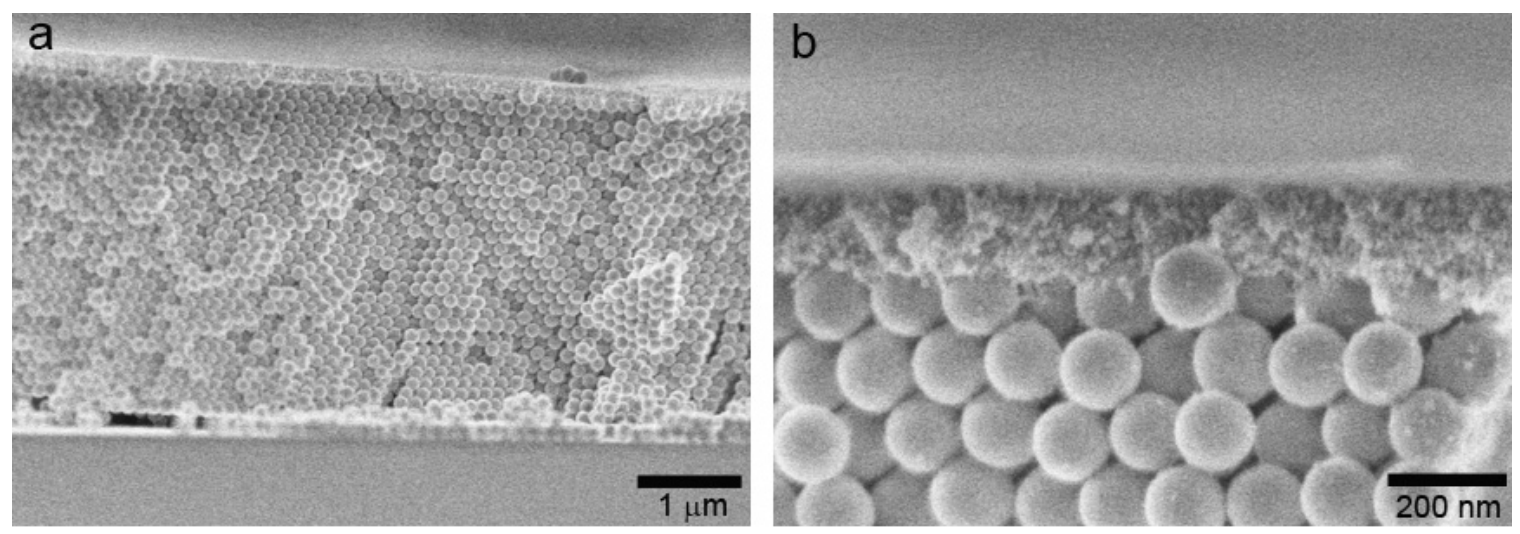

Figure 7.6. SEM images of the bi-layer structure comprising a thin $\mathrm{nc}^{-\mathrm{TiO}_{2}}$ on a $\mathrm{SiO}_{2}$ opal.

Since slow photon and photonic stop-band reflection can enhance the absorption of a photoactive material via different architectures, the combination of the two may achieve the foremost amount of enhancement. Figure 7.7 shows the preliminary results on the fabrication of a bi-layer structure that consists of an absorbing $300-\mathrm{i}-\mathrm{nc}-\mathrm{TiO}_{2}-\mathrm{o}$ on top of a non-absorbing $\mathrm{SiO}_{2}$ opal. The stop-band of the $\mathrm{SiO}_{2}$ opal is positioned at the wavelengths of the slow photon of the $300-\mathrm{i}-\mathrm{nc}-\mathrm{TiO}_{2}-\mathrm{o}$. The slow photon enhances the absorption in the first passage of light while the $\mathrm{SiO}_{2}$ opal reflects light that is not absorbed back into 300-i-nc- $\mathrm{TiO}_{2}-\mathrm{o}$. The amount of slow photon enhancement is magnified in this scheme, and such a structure may be particularly useful for enhancing very weakly absorbing materials. 


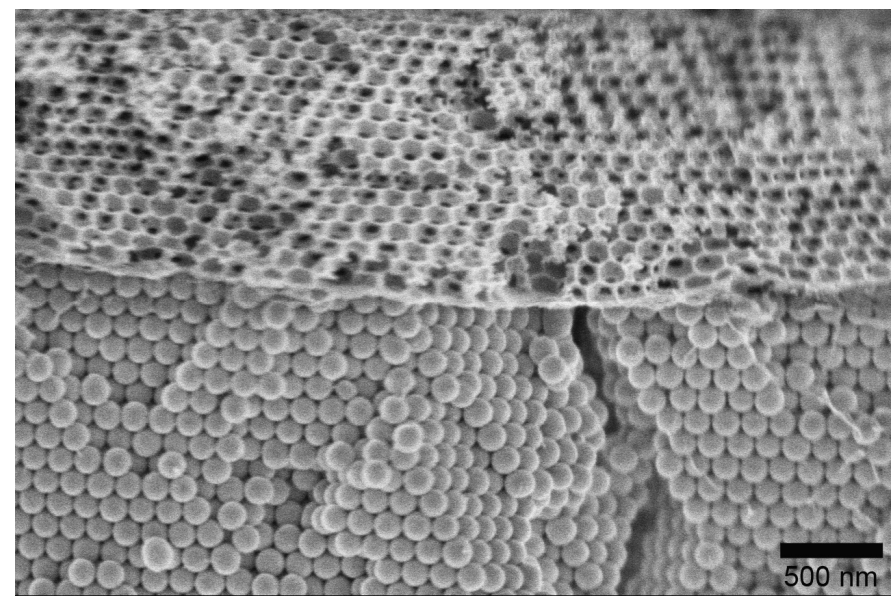

Figure 7.7. SEM image of the proposed bi-layer structure consisting of $300-\mathrm{i}-\mathrm{nc}-\mathrm{TiO}_{2}-\mathrm{o}$ on top of a $\mathrm{SiO}_{2}$ opal.

\subsection{References}

[1] M. Ren, R. Ravikrishna, K. T. Valsaraj, Environ. Sci. Technol. 2006, 40, 7029.

[2] H. Taoda, E. Watanabe, U.S. Patent 5,670,206, 1997.

[3] in www.fresh2.com.

[4] Q. Li, J. K. Shang, J. Am. Ceram. Soc. 2008, 91, 660.

[5] A. Mihi, F. J. Lopez-Alcaraz, H. Miguez, Appl. Phys. Lett. 2006, 88, 193110.

[6] A. Mihi, H. Miguez, J. Phys. Chem. B 2005, 109, 15968.

[7] P. G. O'Brien, N. P. Kherani, S. Zukotynski, G. A. Ozin, E. Vekris, N. Tetreault, A. Chutinan, S. John, A. Mihi, H. Miguez, Adv. Mater. 2007, 19, 4177.

[8] L. I. Halaoui, N. M. Abrams, T. E. Mallouk, J. Phys. Chem. B 2005, 109, 6334.

[9] S. Nishimura, N. Abrams, B. A. Lewis, L. I. Halaoui, T. E. Mallouk, K. D. Benkstein, J. Van de Lagemaat, A. J. Frank, J. Am. Chem. Soc. 2003, 125, 6306. 